TRANSACTIONS OF THE

AMERICAN MATHEMATICAL SOCIETY

Volume 364, Number 3, March 2012, Pages 1293-1367

S 0002-9947(2011)05367-5

Article electronically published on October 12, 2011

\title{
MICKELSSON ALGEBRAS AND REPRESENTATIONS OF YANGIANS
}

\author{
SERGEY KHOROSHKIN AND MAXIM NAZAROV
}

\begin{abstract}
Let $\mathrm{Y}\left(\mathfrak{g l}_{n}\right)$ be the Yangian of the general linear Lie algebra $\mathfrak{g l}_{n}$. We denote by $\mathrm{Y}\left(\mathfrak{s p}_{n}\right)$ and $\mathrm{Y}\left(\mathfrak{s o}_{n}\right)$ the twisted Yangians corresponding to the symplectic and orthogonal subalgebras in the Lie algebra $\mathfrak{g l}_{n}$. These twisted Yangians are one-sided coideal subalgebras in the Hopf algebra $\mathrm{Y}\left(\mathfrak{g l}_{n}\right)$. We provide realizations of irreducible modules of the algebras $\mathrm{Y}\left(\mathfrak{s p}_{n}\right)$ and $\mathrm{Y}\left(\mathfrak{s a}_{n}\right)$ as certain quotients of tensor products of symmetic and exterior powers of the vector space $\mathbb{C}^{n}$. For the Yangian $\mathrm{Y}\left(\mathfrak{g l}_{n}\right)$ such realizations have been known, but we give new proofs of these results. For the twisted Yangian $\mathrm{Y}\left(\mathfrak{s p}_{n}\right)$, we realize all irreducible finite-dimensional modules. For the twisted Yangian $\mathrm{Y}\left(\mathfrak{s o}_{n}\right)$, we realize all those irreducible finite-dimensional modules, where the action of the Lie algebra $\mathfrak{s o}_{n}$ integrates to an action of the special orthogonal Lie group $\mathrm{SO}_{n}$. Our results are based on the theory of reductive dual pairs due to Howe, and on the representation theory of Mickelsson algebras.
\end{abstract}

\section{Preface}

0.1. Brief introduction. Mickelsson algebras are natural objects used for the study of Harish-Chandra modules of real reductive Lie groups. They appeared in [M] first. Their detailed study was later undertaken by Zhelobenko [Z1, Z2, who used the theory of extremal projectors for reductive Lie algebras due to Asherova, Smirnov and Tolstoy AST. Mickelsson algebras were further studied by Ogievetsky and the first author of this article $\mathrm{K}, \mathrm{KO}$. In particular, their works dealt with the extremal cocycle on the Weyl group of any reductive Lie algebra from [Z1].

In Z1 a Mickelsson algebra was defined by a finite-dimensional complex Lie algebra $\mathfrak{a}$ and its reductive subalgebra $\mathfrak{g}$. Following $[\mathrm{K}, \mathrm{KO}$, we define Mickelsson algebras in a more general setting. Let us take any associative algebra A over the complex field $\mathbb{C}$, containing the universal enveloping algebra $U(\mathfrak{g})$ as a subalgebra. Suppose there is a vector subspace $\mathrm{V} \subset \mathrm{A}$, invariant and locally finite under the adjoint action of $\mathfrak{g}$, such that the multiplication map $\mathrm{U}(\mathfrak{g}) \otimes \mathrm{V} \rightarrow \mathrm{A}$ is bijective. Choose a Borel subalgebra of $\mathfrak{g}$ with the Cartan subalgebra $\mathfrak{t}$ and the nilpotent radical $\mathfrak{n}$. Take the right ideal $J=\mathfrak{n} A$ of the algebra $A$ and consider the normalizer Norm $\mathrm{J} \subset \mathrm{A}$ of this ideal. Our Mickelsson algebra $\mathrm{R}$ is the quotient of the algebra Norm J by its two-sided ideal J. In the case when $A=U(\mathfrak{a})$, our $R$ becomes the Mickelsson algebra considered in [Z1].

Let $\overline{\mathrm{A}}$ be the ring of fractions of the algebra $\mathrm{A}$ relative to the set of denominators (1.3). Take the right ideal $\bar{J}=\mathfrak{n} \bar{A}$ of the algebra $\bar{A}$ and its normalizer Norm $\bar{J} \subset \bar{A}$.

Received by the editors November 27, 2009 and, in revised form, April 7, 2010.

2010 Mathematics Subject Classification. Primary 17B35, 81R50.

Key words and phrases. Howe duality, Cherednik functor, Drinfeld functor.

(C)2011 American Mathematical Society Reverts to public domain 28 years from publication 
Then define the algebra $\bar{R}$ as the quotient of Norm $\bar{J}$ by $\bar{J}$. Unlike $R$, the algebra $\bar{R}$ admits a rather nice description. Let $\mathfrak{n}^{\prime}$ be the nilpotent subalgebra of $\mathfrak{g}$ opposite to $\mathfrak{n}$. Let $\mathrm{J}^{\prime}=A \mathfrak{n}^{\prime}$ and $\overline{\mathrm{J}}^{\prime}=\overline{\mathrm{A}} \mathfrak{n}^{\prime}$ be the corresponding left ideals of $\mathrm{A}$ and $\overline{\mathrm{A}}$. Consider the quotient vector space $\overline{\mathrm{Z}}=\overline{\mathrm{A}} /\left(\overline{\mathrm{J}}+\overline{\mathrm{J}}^{\prime}\right)$. Taking the elements of $\overline{\mathrm{R}}$ modulo $\overline{\mathrm{J}}^{\prime}$ defines a map $\overline{\mathrm{R}} \rightarrow \overline{\mathrm{Z}}$. This map is bijective. The multiplication in $\overline{\mathrm{R}}$ can be described in terms of the vector space $\bar{Z}$, by using the extremal projector for $\mathfrak{g}$; see Subsection 1.2. Furthermore, according to [KO], the extremal cocycle corresponding to the Weyl group of $\mathfrak{g}$ determines an action of the braid group of $\mathfrak{g}$ by automorphisms of the algebra $\overline{\mathrm{Z}}$. We call them the Zhelobenko automorphisms of $\bar{Z}$. This braid group action is closely related to the dynamical Weyl group action of Etingof, Tarasov and Varchenko [EV, TV1, TV2].

The adjoint action of the Lie algebra $\mathfrak{g}$ on $\overline{\mathrm{A}}$ determines an action of the Cartan subalgebra $\mathfrak{t} \subset \mathfrak{g}$ on $\bar{Z}$. Consider the subalgebra of $\bar{Z}$ consisting of all elements of zero weight relative to $\mathfrak{t}$. The Zhelobenko automorphisms of $\bar{Z}$ preserve this subalgebra and determine on it an action of the Weyl group of $\mathfrak{g}$, see Subsection 1.4.

Let $\mathrm{G}$ be a reductive algebraic group over $\mathbb{C}$ with the Lie algebra $\mathfrak{g}$. Suppose there is an action of the group $\mathrm{G}$ by automorphisms on the algebra $\mathrm{A}$, extending the adjoint action of $\mathrm{G}$ on the subalgebra $\mathrm{U}(\mathfrak{g}) \subset \mathrm{A}$. Suppose the action of $\mathrm{G}$ on $\mathrm{A}$ preseves the subspace $\mathrm{V}$, and that the action of $\mathrm{G}$ on $\mathrm{V}$ is locally finite. Suppose the action of $\mathfrak{g}$ on $\mathrm{A}$ corresponding to that of $\mathrm{G}$ coincides with the adjoint action. Our joint work with Vinberg [KNV] describes the image Q of the subalgebra of $\mathrm{G}$-invariant elements $\mathrm{A}^{\mathrm{G}} \subset \mathrm{A}$ under the projection $\mathrm{A} \rightarrow \mathrm{A} /\left(\mathrm{J}+\mathrm{J}^{\prime}\right)$. It shows that $\mathrm{Q}$ consists of all elements which have weight zero relative to $t$, are invariant under the Zhelobenko automorphisms of $\overline{\mathrm{A}} /\left(\overline{\mathrm{J}}+\overline{\mathrm{J}}^{\prime}\right)$, and are also invariant under certain other automorphisms arising when the group $G$ is not connected; see Subsection 1.5. Here $\mathrm{A} /\left(\mathrm{J}+\mathrm{J}^{\prime}\right)$ is regarded as a subspace of $\overline{\mathrm{Z}}=\overline{\mathrm{A}} /\left(\overline{\mathrm{J}}+\overline{\mathrm{J}}^{\prime}\right)$. The Zhelobenko automorphisms do not preserve this subspace in general. Thus, using the ring of fractions, $\overline{\mathrm{A}}$ is necessary for this description of $\mathrm{A}^{\mathrm{G}}$.

The present work is a continuation of [KNV] and also of a series of our recent publications [KN1, KN2, KN3, KN4]. The latter series established correspondences between the Zhelobenko automorphisms of certain algebras of the form $\bar{Z}$ discussed above, and canonical intertwining operators of tensor products of representations of Yangians. These are the Yangian $\mathrm{Y}\left(\mathfrak{g l}_{n}\right)$ of the general linear Lie algebra $\mathfrak{g l}_{n}$, and its twisted analogues $\mathrm{Y}\left(\mathfrak{s p}_{n}\right)$ and $\mathrm{Y}\left(\mathfrak{s o}_{n}\right)$ corresponding to the symplectic and orthogonal subalgebras $\mathfrak{s p}_{n}$ and $\mathfrak{s o}_{n}$ of $\mathfrak{g l}_{n}$. For an introduction to the theory of these Yangians, see the book by Molev M2. The Yangian $\mathrm{Y}\left(\mathfrak{g l}_{n}\right)$ is a Hopf algebra while the twisted Yangians $\mathrm{Y}\left(\mathfrak{s p}_{n}\right)$ and $\mathrm{Y}\left(\mathfrak{s o}_{n}\right)$ are one-sided coideal subalgebras of $\mathrm{Y}\left(\mathfrak{g l}_{n}\right)$. These Yangians admit homomorphisms to the rings of the form $\mathrm{A}^{\mathrm{G}}$ discussed above. Moreover, together with the subalgebra $U(\mathfrak{g})^{\mathrm{G}} \subset \mathrm{A}^{\mathrm{G}}$, the image of each of these homomorphisms generates the corresponding ring of invariants $A^{\mathrm{G}}$. These homomorphisms first arose in the works of Olshanski [01, O2] and are also discussed in our Subsection 4.3. Another connection between the works [01, O2] and the theory of Mickelsson algebras was used by Molev to construct weight bases of irreducible finite-dimensional modules of the Lie algebras $\mathfrak{s p}_{n}$ and $\mathfrak{s o}_{n}$; see [M2].

In the present work we combine the results of [KN1, KN2, KN3, KN4 and of KNV to give realizations of irreducible representations of the twisted Yangians. In particular, every irreducible finite-dimensional module of the twisted Yangian $\mathrm{Y}\left(\mathfrak{s p}_{n}\right)$ will be realized, up to changing the action of the centre of this algebra, as 
the image of an intertwining operator between representations of $\mathrm{Y}\left(\mathfrak{s p}_{n}\right)$ in tensor products of exterior powers of $\mathbb{C}^{n}$. For the Yangian $\mathrm{Y}\left(\mathfrak{g l}_{n}\right)$, such realizations were provided by Akasaka and Kashiwara [AK] and Cherednik [C2]. We give new proofs of these results for $\mathrm{Y}\left(\mathfrak{g l}_{n}\right)$. For the twisted Yangian $\mathrm{Y}\left(\mathfrak{s o}_{n}\right)$, the images of our intertwining operators realize, up to changing the action of the centre of $\mathrm{Y}\left(\mathfrak{s o}_{n}\right)$, all those irreducible finite-dimensional modules, where the action of the subalgebra $\mathrm{U}\left(\mathfrak{s o}_{n}\right) \subset \mathrm{Y}\left(\mathfrak{s o}_{n}\right)$ integrates to an action of the special orthogonal Lie group $\mathrm{SO}_{n}$.

Now let $\mathrm{U}(\mathfrak{g}) \subset \mathrm{A}$ be an arbitrary pair as described above. Let $\mathrm{A}^{0}$ be the zero weight component of the algebra $A$ relative to the adjoint action of $t$. We denote by $\mathrm{S}$ the subalgebra in $\overline{\mathrm{Z}}$ generated by the image of $\mathrm{A}^{0} \subset \overline{\mathrm{A}}$ under the projection $\overline{\mathrm{A}} \rightarrow \overline{\mathrm{Z}}$. Then $\mathrm{Q} \subset \mathrm{S}$ by definition. For any weight $\lambda \in \mathfrak{t}^{*}$, let $N$ be an irreducible $\mathrm{S}$-module whereon the subalgebra $\mathrm{U}(\mathfrak{t}) \subset \mathrm{S}$ acts via the mapping $\lambda: \mathfrak{t} \rightarrow \mathbb{C}$, extended to a homomorphism $\mathrm{U}(\mathfrak{t}) \rightarrow \mathbb{C}$. Then our Proposition 1.6 gives sufficient conditions for irreducibility of the restriction of $N$ to the subalgebra $\mathrm{Q} \subset \mathrm{S}$. One of the conditions is that the weight $\lambda+\rho$ is dominant. Here $\rho$ denotes the half-sum of positive roots, and the dominance means that the value of $\lambda+\rho$ on any positive coroot vector of $\mathfrak{t}$ is not a negative integer.

Our results on irreducibility of representations of the Yangians $\mathrm{Y}\left(\mathfrak{g l}_{n}\right), \mathrm{Y}\left(\mathfrak{s p}_{n}\right)$, and $\mathrm{Y}\left(\mathfrak{s o}_{n}\right)$ are based on Proposition [1.6. By using the homomorphism $\mathrm{A}^{\mathrm{G}} \rightarrow \mathrm{Q}$ determined by the projection $\mathrm{A} \rightarrow \mathrm{A} /\left(\mathrm{J}+\mathrm{J}^{\prime}\right)$, any irreducible Q-module becomes an irreducible module over the algebra $\mathrm{A}^{\mathrm{G}}$. By using the homomorphisms from Yangians to algebras of the form $\mathrm{A}^{\mathrm{G}}$, we get irreducible representations of Yangians.

Proposition [1.6 is the main result of Section 1. In that section we also collect several other results on the algebra $\overline{\mathrm{Z}}$ corresponding to an arbitrary pair $\mathrm{U}(\mathfrak{g}) \subset \mathrm{A}$. Below we describe the contents of all other sections of our article in more detail.

0.2. Overview of Section 2. In this article, we employ the theory of reductive dual pairs due to Howe [H1. Namely, we use the pairs $\left(G, G^{\prime}\right)$ of complex reductive algebraic groups from the following list:

$$
\left(\mathrm{GL}_{m}, \mathrm{GL}_{n}\right),\left(\mathrm{O}_{2 m}, \mathrm{Sp}_{n}\right),\left(\mathrm{Sp}_{2 m}, \mathrm{O}_{n}\right),\left(\mathrm{Sp}_{2 m}, \mathrm{Sp}_{n}\right),\left(\mathrm{O}_{2 m}, \mathrm{O}_{n}\right) \text {. }
$$

It is the Lie algebra $\mathfrak{g}^{\prime}$ of the group $G^{\prime}$ dual to $G$ that determines the Yangian $Y\left(\mathfrak{g}^{\prime}\right)$. We will employ both the symmetric and skew-symmetric versions of the Howe duality. Let $U$ be the tensor product $\mathbb{C}^{m} \otimes \mathbb{C}^{n}$ of vector spaces. The group $\mathrm{G}^{\prime}=\mathrm{GL}_{n}, \mathrm{Sp}_{n}, \mathrm{O}_{n}$ acts on $U$ naturally, that is, via its defining action on $\mathbb{C}^{n}$. In the symmetric case, this action of $\mathrm{G}^{\prime}$ is extended from $U$ to the Weyl algebra of $U$. The subalgebra of $\mathrm{G}^{\prime}$-invariant elements in the Weyl algebra then becomes a homomorphic image of the universal enveloping algebra $U(\mathfrak{g})$, where $\mathfrak{g}$ is the Lie algebra of $\mathrm{G}=\mathrm{GL}_{m}, \mathrm{O}_{2 m}, \mathrm{Sp}_{2 m}$, respectively. In the skew-symmetric case, the action of $\mathrm{G}^{\prime}$ on $U$ is extended from $U$ to the Clifford algebra of $U$. Then the subalgebra of $\mathrm{G}^{\prime}$-invariant elements in the Clifford algebra becomes a homomorphic image of $\mathrm{U}(\mathfrak{g})$ where $\mathfrak{g}$ is the Lie algebra of $\mathrm{G}=\mathrm{GL}_{m}, \mathrm{Sp}_{2 m}, \mathrm{O}_{2 m}$, respectively. Thus the first pair in the list (0.1) appears in both the symmetric and skew-symmetric cases, the next two pairs appear only in the symmetric case, while the last two pairs appear only in the skew-symmetric case.

We will denote by $\mathrm{H}(U)$ either the Weyl or the Clifford algebra, and distinguish the symmetric and the skew-symmetric cases by using a parameter $\theta$ which equals 1 or -1 , respectively. The homomorphism $\mathrm{U}(\mathfrak{g}) \rightarrow \mathrm{H}(U)$ will be denoted by $\zeta$. Our algebra A will be the tensor product $\mathrm{U}(\mathfrak{g}) \otimes \mathrm{H}(U)$. The algebra $\mathrm{U}(\mathfrak{g})$ will be 
embedded into this tensor product diagonally: any element $X \in \mathfrak{g}$ will be identified with the element (2.6) of A. The subspace $\mathrm{V} \subset \mathrm{A}$ will then be $1 \otimes \mathrm{H}(U)$. The group $\mathrm{G}$ acts by automorphisms of the algebra $\mathrm{H}(U)$, and the corresponding action of $\mathfrak{g}$ on $\mathrm{H}(U)$ coincides with the adjoint to $\zeta$. Thus we get a (diagonal) action of the group $G$ by automorphisms of the algebra $A$, obeying the assumptions from the beginning of this section. In Subsection 2.1 we summarize the properties of the homomorphism $\zeta: \mathrm{U}(\mathfrak{g}) \rightarrow \mathrm{H}(U)$ that we will use.

In Subsection 2.2 we introduce our main tool, the Shapovalov form on the algebra $\overline{\mathrm{Z}}$ corresponding to $\mathrm{A}=\mathrm{U}(\mathfrak{g}) \otimes \mathrm{H}(U)$. It takes values in the ring of fractions $\overline{\mathrm{U}(\mathfrak{t})}$ of the algebra $U(t)$ relative to the set of denominators (1.3). The algebra $\mathrm{H}(U)$ is generated by its two subspaces, $U$ and the dual $U^{*}$. Let $\mathrm{P}(U)$ be the subalgebra of $\mathrm{H}(U)$ generated by the subspace $U$. Then $\mathrm{P}(U)$ is the symmetric algebra of $U$ when $\theta=1$, or the exterior algebra of $U$ when $\theta=-1$. For any $\mu \in \mathfrak{t}^{*}$, let $M_{\mu}$ be the corresponding Verma module of $\mathfrak{g}$. There is a unique weight $\kappa \in \mathfrak{t}^{*}$ satisfying (2.12). This weight is zero if $\mathfrak{g}=\mathfrak{g l}_{m}$, but differs from zero if $\mathfrak{g}=\mathfrak{s p}_{2 m}$ or $\mathfrak{g}=\mathfrak{s o}_{2 m}$.

In Subsection 2.3 we take the space of $\mathfrak{n}$-coinvariants of $\mathfrak{g}$-module $M_{\mu} \otimes \mathrm{P}(U)$. This space is denoted by $\mathrm{M}_{\mu}$. It is identified with a quotient space of the algebra A by the right ideal $\mathrm{J}$ and by a certain left ideal depending on $\mu$; see the definition (2.14). If the weight $\mu+\kappa$ is generic, that is, if the value of $\mu+\kappa$ on any coroot vector of $\mathfrak{t}$ is not an integer, then the $\overline{\mathrm{U}(\mathfrak{t})}$-valued Shapovalov form on $\overline{\mathrm{Z}}$ defines a $\mathbb{C}$-valued bilinear form on $\mathrm{M}_{\mu}$. The latter form is denoted by $S_{\mu}$. Proposition 2.3 relates the form $S_{\mu}$ to the extremal projector for the Lie algebra $\mathfrak{g}$.

In Subsection 2.4 for any $\lambda \in \mathfrak{t}^{*}$ we take the subspace of weight $\lambda$ in the space $\mathrm{M}_{\mu}$. This subspace is denoted by $\mathrm{M}_{\mu}^{\lambda}$. If $\mu+\kappa$ is generic, then we define a $\mathbb{C}$ valued bilinear form $S_{\mu}^{\lambda}$ on the subspace $\mathrm{M}_{\mu}^{\lambda} \subset \mathrm{M}_{\mu}$ as the restriction of the form $S_{\mu}$. Letting the weights $\lambda$ and $\mu$ vary while the difference $\lambda-\mu$ is fixed, we extend the definition of $S_{\mu}^{\lambda}$ to all $\mu$ such that $\lambda+\rho$ is dominant, by continuity. Thus here $\mu+\kappa$ needs not be generic. This extension of $S_{\mu}^{\lambda}$ corresponds to the fusion procedure of Cherednik [C2]. Our Proposition 2.6 states that the quotient of $\mathrm{M}_{\mu}^{\lambda}$ by the kernel of the bilinear form $S_{\mu}^{\lambda}$ is an irreducible S-module. This is the main result of Section 2. It shows that under the extra conditions of Proposition 1.6 the quotient of $\mathrm{M}_{\mu}^{\lambda}$ by the kernel of $S_{\mu}^{\lambda}$ is an irreducible Q-module; see Corollary 2.7

In Subsection 2.5 we give another construction of irreducible Q-modules, by using the general results on the Mickelsson algebras from our Subsection 1.6. Let $L_{\mu}$ be the quotient of the Verma module $M_{\mu}$ by its maximal proper submodule $N_{\mu}$. Take the space of $\mathfrak{n}$-coinvariants of weight $\lambda$ of the $\mathfrak{g}$-module $L_{\mu} \otimes \mathrm{P}(U)$. We denote this space by $\mathrm{L}_{\mu}^{\lambda}$. If $\lambda+\rho$ is dominant, then $\mathrm{L}_{\mu}^{\lambda}$ is an irreducible $\mathrm{S}$-module. Moreover, then under the extra conditions of Proposition 1.6, the space $\mathrm{L}_{\mu}^{\lambda}$ is an irreducible Q-module; see Corollary 2.8. Note that then $\mathrm{L}_{\mu}^{\lambda}$ is an irreducible $\mathrm{A}^{\mathrm{G}}$ module by Proposition [1.5. Thus we extend the results of Harish-Chandra [H] and of Lepowski and McCollum [LM].

Now take the space of $\mathfrak{n}$-coinvariants of weight $\lambda$ of the $\mathfrak{g}$-module $N_{\mu} \otimes \mathrm{P}(U)$. We denote this space by $\mathrm{N}_{\mu}^{\lambda}$. Then $\mathrm{L}_{\mu}^{\lambda}$ can be regarded as the quotient of the space of $\mathrm{M}_{\mu}^{\lambda}$ by its subspace $\mathrm{N}_{\mu}^{\lambda}$. In the case when $\lambda+\rho$ is dominant while $\mu$ satisfies the conditions of Proposition 2.9, the subspace $\mathrm{N}_{\mu}^{\lambda} \subset \mathrm{M}_{\mu}^{\lambda}$ coincides with the kernel of the form $S_{\mu}^{\lambda}$. Hence the quotient of $\mathrm{M}_{\mu}^{\lambda}$ by the kernel of $S_{\mu}^{\lambda}$ coincides with $\mathrm{L}_{\mu}^{\lambda}$ in this case. For each pair $\left(\mathrm{G}, \mathrm{G}^{\prime}\right)$ from the list (0.1), weights $\mu$ satisfying the conditions of Proposition 2.9 will be produced later on, in Section 4. 
0.3. Overview of Section 3. In Section 3 we give another interpretation of the quotient space of $\mathrm{M}_{\mu}^{\lambda}$ by the kernel of $S_{\mu}^{\lambda}$, for any $\mu$ and dominant $\lambda+\rho$. The extremal cocycle corresponding to the Weyl group $\mathfrak{S}$ of $\mathfrak{g}$ determines not only an action of the braid group of $\mathfrak{g}$ by automorphisms of the algebra $\bar{Z}$, but also an action of the same braid group by linear operators on the vector space $\overline{\mathrm{A}} / \overline{\mathrm{J}}$. We call them the Zhelobenko operators on $\overline{\mathrm{A}} / \overline{\mathrm{J}}$; see Subsection 1.4. Let the symbol $\circ$ indicate the shifted action of the group $\mathfrak{S}$ on $\mathfrak{t}^{*}$; see (1.2). Again suppose that $\mu+\kappa$ is generic. Then the quotient space $\mathrm{M}_{\mu}$ of $\mathrm{A}$ can be identified with the quotient space of $\bar{A}$ by the right ideal $\bar{J}$ and by a certain left ideal. In Subsection 3.1 we describe the image of the quotient $\mathrm{M}_{\mu}$ of $\overline{\mathrm{A}}$ under the action of the Zhelobenko operator $\check{\xi}_{\sigma}$ on $\overline{\mathrm{A}} / \overline{\mathrm{J}}$, corresponding to any element $\sigma \in \mathfrak{S}$. If $\mathfrak{g}=\mathfrak{g l}_{m}$, then this image can be identified with $\mathrm{M}_{\sigma \circ \mu}$. But if $\mathfrak{g}=\mathfrak{s p}_{2 m}$ or $\mathfrak{g}=\mathfrak{s o}_{2 m}$, then our description of the image is more involved; see Corollary [3.4. For $\mathfrak{g}=\mathfrak{g l}_{m}$ this result was obtained in [KN1, KN2 . For $\mathfrak{g}=\mathfrak{s p}_{2 m}$ or $\mathfrak{g}=\mathfrak{s o}_{2 m}$ it was obtained in KN3, KN4. In Subsection 3.1 we give a new proof of this result, uniform for all these $\mathfrak{g}$.

For generic $\mu+\kappa$ the operator $\check{\xi}_{\sigma}$ on $\overline{\mathrm{A}} / \overline{\mathrm{J}}$ maps the subspace $\mathrm{M}_{\mu}^{\lambda} \subset \mathrm{M}_{\mu}$ of weight $\lambda$ to the subspace of weight $\sigma \circ \lambda$ in the image of $\mathrm{M}_{\mu}$. Our Proposition 3.5 relates this map to the extremal projector for $\mathfrak{g}$. Letting the weights $\lambda$ and $\mu$ vary while their difference $\lambda-\mu$ is fixed, we extend the definition of this map to all $\mu$ such that $\lambda+\rho$ is dominant, again by continuity; see Corollary 3.6. Thus the weight $\mu+\kappa$ does not need to be generic anymore. Yet if $\mu+\kappa$ is not generic, then the operator $\check{\xi}_{\sigma}$ on $\overline{\mathrm{A}} / \overline{\mathrm{J}}$ does not necessarily define any map on the whole space $\mathrm{M}_{\mu}$. In Subsection 3.3 we consider the operator $\check{\xi}_{\sigma_{0}}$ where $\sigma_{0}$ is the longest element of $\mathfrak{S}$. Our Proposition 3.7 states that the kernel of the corresponding map of $\mathrm{M}_{\mu}^{\lambda}$ coincides with the kernel of the bilinear form $S_{\mu}^{\lambda}$, for any $\mu$ and dominant $\lambda+\rho$. The proof is based on Propositions 2.3 and 3.5. Corollary 2.7 then implies that the quotient space of $\mathrm{M}_{\mu}^{\lambda}$ by the kernel of the map defined by $\check{\xi}_{\sigma_{0}}$ is an irreducible Q-module, under the extra conditions of Proposition 1.6. Moreover, then by Proposition 1.5 this quotient space of $\mathrm{M}_{\mu}^{\lambda}$ is an irreducible $\mathrm{A}^{\mathrm{G}}$-module. These are the main results of Section 3; they are stated as Theorem 3.8 and Corollary 3.9 .

0.4. Overview of Section 4. In Section 4 we apply the results of Section 3 to the representation theory of the Yangians $\mathrm{Y}\left(\mathfrak{g}^{\prime}\right)$ where $\mathfrak{g}^{\prime}=\mathfrak{g l}_{n}, \mathfrak{s p}_{n}, \mathfrak{s o}_{n}$ according to the list (0.1). In Subsection 4.1 we fix realizations of the groups $G$ and $G^{\prime}$ appearing in (0.1) and define the corresponding homomorphisms $\zeta: \mathrm{U}(\mathfrak{g}) \rightarrow \mathrm{H}(U)$. In Subsection 4.2 we recall the definitions of the Yangian $Y\left(\mathfrak{g l}_{n}\right)$ and of the twisted Yangians $\mathrm{Y}\left(\mathfrak{s p}_{n}\right), \mathrm{Y}\left(\mathfrak{s o}_{n}\right)$. The latter two are defined as some subalgebras of $\mathrm{Y}\left(\mathfrak{g l}_{n}\right)$. Their definition implies that they are also right coideals of $\mathrm{Y}\left(\mathrm{gl}_{n}\right)$; see (4.22).

The twisted Yangians $\mathrm{Y}\left(\mathfrak{s p}_{n}\right), \mathrm{Y}\left(\mathfrak{s o}_{n}\right)$ can also be defined in terms of generators and relations; see (4.2) and (4.18). The collection of relations (4.2) can be written as the reflection equation, due to Cherednik [C1] and Sklyanin [S]. The algebras defined by the relations (4.2) only, that is, without the relations (4.18), are called the extended twisted Yangians. We denote them by $\mathrm{X}\left(\mathfrak{s p}_{n}\right), \mathrm{X}\left(\mathfrak{s o}_{n}\right)$, respectively. Thus for $\mathfrak{g}^{\prime}=\mathfrak{s p}_{n}, \mathfrak{s 0 _ { n }}$ we have a surjective homomorphism $\mathrm{X}\left(\mathfrak{g}^{\prime}\right) \rightarrow \mathrm{Y}\left(\mathfrak{g}^{\prime}\right)$. Its kernel is generated by certain central elements of $\mathrm{X}\left(\mathfrak{g}^{\prime}\right)$; see Subsection 4.2.

In Proposition 4.1 for $\mathrm{A}=\mathrm{U}\left(\mathfrak{g l}_{m}\right) \otimes \mathrm{H}(U)$ and any $\theta=1$, -1 , we will define a homomorphism $\mathrm{Y}\left(\mathfrak{g l}_{n}\right) \rightarrow \mathrm{A}^{\mathrm{GL}_{m}}$. Its image and the subalgebra $\mathrm{U}\left(\mathfrak{g l}_{m}\right)^{\mathrm{GL}_{m}} \otimes 1 \subset \mathrm{A}$ together generate $\mathrm{A}^{\mathrm{GL}_{m}}$. It is related to the homomorphism $\mathrm{Y}\left(\mathfrak{g l}_{n}\right) \rightarrow \mathrm{U}\left(\mathfrak{g l}_{n+l}\right)^{\mathrm{GL}_{l}}$ 
defined for $l=1,2, \ldots$ by Olshanski [O1]. It is also related to the Cherednik functor studied by Arakawa and Suzuki [AS] and to the Drinfeld functor [D1]. We explained the relations in [KN1, KN2].

Now consider any pair $\left(\mathrm{G}, \mathrm{G}^{\prime}\right)$ from the list (0.1) other than the pair $\left(\mathrm{GL}_{m}, \mathrm{GL}_{n}\right)$. In Proposition 4.2 for $\mathrm{A}=\mathrm{U}(\mathfrak{g}) \otimes \mathrm{H}(U)$, we define a homomorphism $\mathrm{X}\left(\mathfrak{g}^{\prime}\right) \rightarrow \mathrm{A}^{\mathrm{G}}$. Its image together with the subalgebra $\mathrm{U}(\mathfrak{g})^{\mathrm{G}} \otimes 1 \subset \mathrm{A}$ generates $\mathrm{A}^{\mathrm{G}}$. It is related to the homomorphisms $\mathrm{Y}\left(\mathfrak{s p}_{n}\right) \rightarrow \mathrm{U}\left(\mathfrak{s p}_{n+2 l}\right) \mathrm{Sp}_{2 l}$ and $\mathrm{Y}\left(\mathfrak{s o}_{n}\right) \rightarrow \mathrm{U}\left(\mathfrak{s o}_{n+l}\right)^{\mathrm{O}_{l}}$ defined for any $l=1,2, \ldots$ by Olshanski [O2]. We explained the relation in [KN3, KN4]. To prove our Propositions 4.1 and 4.2 we use classical invariant theory, as Molev and Olshanski [MO] did when studying the homomorphisms defined in [01, 02].

For any $\mathfrak{g}^{\prime}=\mathfrak{g l}_{n}, \mathfrak{s p}_{n}, \mathfrak{s o}_{n}$ the algebra $\mathrm{Y}\left(\mathfrak{g}^{\prime}\right)$ contains $\mathrm{U}\left(\mathfrak{g}^{\prime}\right)$ as a subalgebra and admits a homomorphism $\mathrm{Y}\left(\mathfrak{g}^{\prime}\right) \rightarrow \mathrm{U}\left(\mathfrak{g}^{\prime}\right)$ identical on that subalgebra. For $\mathfrak{g}^{\prime}=\mathfrak{g l}_{n}$ this fact has been well known; see for instance the seminal work of Kulish, Reshetikhin and Sklyanin [KRS. For $\mathfrak{g}^{\prime}=\mathfrak{s p}_{n}, \mathfrak{s o}_{n}$ this is another result from [2]. If $\theta=1$, then denote by $\Phi^{k}$ the $k$ th symmetric power of the defining $\mathfrak{g l}_{n}$-module $\mathbb{C}^{n}$. If $\theta=-1$, then denote by the same symbol $\Phi^{k}$ the $k$ th exterior power of the $\mathfrak{g l}_{n}$-module $\mathbb{C}^{n}$. Using the homomorphism $\mathrm{Y}\left(\mathfrak{g l}_{n}\right) \rightarrow \mathrm{U}\left(\mathfrak{g l}_{n}\right)$, regard $\Phi^{k}$ as a module over $\mathrm{Y}\left(\mathfrak{g l}_{n}\right)$. For any $t \in \mathbb{C}$ denote by $\Phi_{t}^{k}$ the $\mathrm{Y}\left(\mathfrak{g l}_{n}\right)$-module obtained by pulling the $\mathrm{Y}\left(\mathfrak{g l}_{n}\right)$-module $\Phi^{k}$ back through the automorphism (4.6) where $z=\theta t$.

The definition of the Hopf algebra $\mathrm{Y}\left(\mathfrak{g l}_{n}\right)$ employs a certain $n \times n$ matrix $T(x)$. The entries of this matrix are formal power series in $x^{-1}$ where $x$ is the spectral parameter. The coefficients of these series are generators of $\mathrm{Y}\left(\mathfrak{g l}_{n}\right)$. For $\mathrm{G}^{\prime}=\mathrm{Sp}_{n}$ or $\mathrm{G}^{\prime}=\mathrm{O}_{n}$, we denote by $\widetilde{T}(x)$ the transpose to the matrix $T(x)$ relative to the bilinear form on $\mathbb{C}^{n}$ preserved by the subgroup $\mathrm{G}^{\prime} \subset \mathrm{GL}_{n}$. The assignment (4.12) defines an involutive automorphism of the algebra $\mathrm{Y}\left(\mathfrak{g l}_{n}\right)$. For any nonnegative integer $k$, we denote by $\Phi_{t}^{-k}$ the $\mathrm{Y}\left(\mathfrak{g l}_{n}\right)$-module obtained by pulling the $\Phi_{t}^{k}$ back through the automorphism (4.12).

For $\mathfrak{g}^{\prime}=\mathfrak{s p}_{n}, \mathfrak{s o}_{n}$ the subalgebra $\mathrm{Y}\left(\mathfrak{g}^{\prime}\right) \subset \mathrm{Y}\left(\mathfrak{g l}_{n}\right)$ is generated by the coefficients of the series in $x$ which arise as the entries of the $n \times n$ matrix $\widetilde{T}(-x) T(x)$. It turns out that if $\mathfrak{g}^{\prime}=\mathfrak{s p}_{n}$ and $\theta=1$, or if $\mathfrak{g}^{\prime}=\mathfrak{s o} \mathfrak{o}_{n}$ and $\theta=-1$, then the restriction of the above-defined $\mathrm{Y}\left(\mathfrak{g l}_{n}\right)$-module $\Phi_{t}^{-k}$ to the subalgebra $\mathrm{Y}\left(\mathfrak{g}^{\prime}\right) \subset \mathrm{Y}\left(\mathfrak{g l}_{n}\right)$ coincides with the restriction of the $\mathrm{Y}\left(\mathfrak{g l}_{n}\right)$-module $\Phi_{t}^{k}$. Both cases correspond to $\mathfrak{g}=\mathfrak{s o}_{2 m}$. For further explanation of this phenomenon, see Subsection 4.4.

For any pair $\left(\mathrm{G}, \mathrm{G}^{\prime}\right)$ from (0.1) and any weights $\lambda, \mu \in \mathfrak{t}^{*}$, the subspace $\mathrm{M}_{\mu}^{\lambda}$ of the quotient $\mathrm{M}_{\mu}$ of the algebra $\mathrm{A}$ is a module over the subalgebra $\mathrm{A}^{\mathrm{G}}$ by definition. For $\left(\mathrm{G}, \mathrm{G}^{\prime}\right)=\left(\mathrm{GL}_{m}, \mathrm{GL}_{n}\right)$, by using the homomorphism $\mathrm{Y}\left(\mathfrak{g l}_{n}\right) \rightarrow \mathrm{A}^{\mathrm{GL}_{m}}$, we can regard $\mathrm{M}_{\mu}^{\lambda}$ as a module over the algebra $\mathrm{Y}\left(\mathfrak{g l}_{n}\right)$. If nonzero, this $\mathrm{Y}\left(\mathfrak{g l}_{n}\right)$-module is equivalent to a tensor product of certain modules of the form $\Phi_{t}^{k}$. This is stated as Proposition 4.3, see [KN1, KN2 for the proof. Here we use the comultiplication (4.10) on $\mathrm{Y}\left(\mathfrak{g l}_{n}\right)$.

For any other pair $\left(\mathrm{G}, \mathrm{G}^{\prime}\right)$ from the list (0.1), we can regard $\mathrm{M}_{\mu}^{\lambda}$ as a module of the algebra $\mathrm{X}\left(\mathfrak{g}^{\prime}\right)$ by using the homomorphism $\mathrm{X}\left(\mathfrak{g}^{\prime}\right) \rightarrow \mathrm{A}^{\mathrm{G}}$. If nonzero, this $\mathrm{X}\left(\mathfrak{g}^{\prime}\right)$ module is also equivalent to a tensor product of certain modules of the form $\Phi_{t}^{k}$, pulled back through an automorphism 4.15) of $\mathrm{X}\left(\mathfrak{g}^{\prime}\right)$. This is a particular case of our Proposition 4.5, see [KN3, KN4 for the proof of that proposition. Here we first take the tensor product of the $\mathrm{Y}\left(\mathfrak{g l}_{n}\right)$-modules $\Phi_{t}^{k}$, then restrict the tensor product to the subalgebra $\mathrm{Y}\left(\mathfrak{g}^{\prime}\right) \subset \mathrm{Y}\left(\mathfrak{g l}_{n}\right)$, and then use the homomorphism $\mathrm{X}\left(\mathfrak{g}^{\prime}\right) \rightarrow \mathrm{Y}\left(\mathfrak{g}^{\prime}\right)$ 
mentioned above. Equivalently, we can use the right $\mathrm{Y}\left(\mathfrak{g l}_{n}\right)$-comodule structure on the algebra $\mathrm{X}\left(\mathfrak{g}^{\prime}\right)$; see Subsection 4.2.

Now suppose that $\lambda+\rho$ is dominant while $\mu$ is arbitrary. Consider again the mapping of the weight subspace $\mathrm{M}_{\mu}^{\lambda}$ defined by the Zhelobenko operator $\check{\xi}_{\sigma_{0}}$ on $\overline{\mathrm{A}} / \overline{\mathrm{J}}$. In the case $\left(\mathrm{G}, \mathrm{G}^{\prime}\right)=\left(\mathrm{GL}_{m}, \mathrm{GL}_{n}\right)$, by replacing $\mathrm{M}_{\mu}^{\lambda}$ by an equivalent $\mathrm{Y}\left(\mathfrak{g l}_{n}\right)$-module, we obtain an intertwining operator from a tensor product of $\mathrm{Y}\left(\mathfrak{g l}_{n}\right)$-modules of the form $\Phi_{t}^{k}$. The target module of this operator can be identified with the tensor product of the same $\mathrm{Y}\left(\mathfrak{g l}_{n}\right)$-modules $\Phi_{t}^{k}$ as for the source module, but taken in reverse order; see (4.49). The quotient by the kernel, or equivalently the image of this intertwining operator, is an irreducible $\mathrm{Y}\left(\mathfrak{g l}_{n}\right)$-module due to Corollary 3.9 and Proposition 4.1

In the case $\left(\mathrm{G}, \mathrm{G}^{\prime}\right)=\left(\mathrm{GL}_{m}, \mathrm{GL}_{n}\right)$, we can regard the quotient space $\mathrm{L}_{\mu}^{\lambda}$ of $\mathrm{M}_{\mu}^{\lambda}$ as an $\mathrm{Y}\left(\mathfrak{g l}_{n}\right)$-module. The Yangian $\mathrm{Y}\left(\mathfrak{g l}_{n}\right)$ acts on $\mathrm{M}_{\mu}^{\lambda}$ and hence on $\mathrm{L}_{\mu}^{\lambda}$ via the homomorphism $\mathrm{Y}\left(\mathfrak{g l}_{n}\right) \rightarrow \mathrm{A}^{\mathrm{GL}_{m}}$. If $\lambda+\rho$ is dominant, then the $\mathrm{Y}\left(\mathfrak{g l}_{n}\right)$-module $\mathrm{L}_{\mu}^{\lambda}$ is irreducible for any $\mu$; see Subsection 4.6. Moreover, then the conditions of Proposition 2.9 are satisfied if for each positive root $\alpha$ of $\mathfrak{g l}_{m}$ the number $z_{\alpha}$ (defined just before stating Proposition (4.4) is not zero. Under these conditions, the image of our intertwining operator (4.49) is not zero and is equivalent to $\mathrm{L}_{\mu}^{\lambda}$ as a $\mathrm{Y}\left(\mathfrak{g l}_{n}\right)$-module. Thus we extend the results of $\mathrm{AS}$.

Now take a pair $\left(\mathrm{G}, \mathrm{G}^{\prime}\right)$ from (0.1) other than $\left(\mathrm{GL}_{m}, \mathrm{GL}_{n}\right)$. By replacing $\mathrm{M}_{\mu}^{\lambda}$ by an equivalent $\mathrm{X}\left(\mathfrak{g}^{\prime}\right)$-module, we again obtain an intertwining operator from a certain tensor product of modules of the form $\Phi_{t}^{k}$. The target module of this operator can be identified with the tensor product of the modules $\Phi_{t}^{-k}$ corresponding to the tensor factors $\Phi_{t}^{k}$ of the source module and is taken in the same order; see (4.58). Here we need not apply to the source and the target $\mathrm{X}\left(\mathfrak{g}^{\prime}\right)$-modules the automorphisms (4.15) because by Proposition 4.5 these automorphisms are the same for the source and for the target. Hence we may also regard both the source and the target as $\mathrm{Y}\left(\mathfrak{g}^{\prime}\right)$-modules. Note that if $\mathfrak{g}=\mathfrak{s o}_{2 m}$, then instead of the first tensor factor $\Phi_{t}^{-k}$ of the target we can use $\Phi_{t}^{k}$ as well; see (4.59). This is because for $\mathfrak{g}=\mathfrak{s o}_{2 m}$ the restrictions of $\Phi_{t}^{k}$ and $\Phi_{t}^{-k}$ to the subalgebra $\mathrm{Y}\left(\mathfrak{g}^{\prime}\right) \subset \mathrm{Y}\left(\mathfrak{g l}_{n}\right)$ coincide, and because we regard this subalgebra as a right coideal to define the tensor products.

If $\mathrm{G}=\mathrm{Sp}_{2 m}$, then by Corollary 3.9 and Proposition 4.2 the image of operator (4.58) is an irreducible $Y\left(\mathfrak{g}^{\prime}\right)$-module for both $\theta=1$ and $\theta=-1$, that is, for both $\mathfrak{g}^{\prime}=\mathfrak{s o}_{n}$ and $\mathfrak{g}^{\prime}=\mathfrak{s p}_{n}$. But if $\mathrm{G}=\mathrm{O}_{2 m}$, then our Corollary 3.9 and Proposition 4.2 imply $\mathrm{Y}\left(\mathfrak{g}^{\prime}\right)$-irreducibility of the image only under an extra condition, that the stabilizer of the weight $\lambda+\rho$ in the extended Weyl group of $\mathfrak{g}=\mathfrak{s o}_{2 m}$ is contained in the proper Weyl group. In Subsection 4.5 we remove this extra condition, but only for $\theta=-1$, that is, only for $\mathfrak{g}^{\prime}=\mathfrak{s o}_{n}$. Namely, for $\mathrm{G}=\mathrm{O}_{2 m}$ and $\theta=-1$, we prove that the image of our intertwining operator is irreducible under the joint action of the algebra $\mathrm{Y}\left(\mathfrak{s o}_{n}\right)$ and the group $\mathrm{O}_{n}$. Moreover, if $n$ is odd, then the image is irreducible under the action of $\mathrm{Y}\left(\mathfrak{s o}_{n}\right)$ alone. But if $n$ is even, then the image is either an irreducible $\mathrm{Y}\left(\mathfrak{s o}_{n}\right)$-module or is a direct sum of two nonequivalent irreducible $\mathrm{Y}\left(\mathfrak{s o}_{n}\right)$-modules; see Corollary 4.9 .

Now let $\left(\mathrm{G}, \mathrm{G}^{\prime}\right)$ again be any pair from (0.1) other than $\left(\mathrm{GL}_{m}, \mathrm{GL}_{n}\right)$. Regard the quotient space $\mathrm{L}_{\mu}^{\lambda}$ of $\mathrm{M}_{\mu}^{\lambda}$ as an $\mathrm{X}\left(\mathfrak{g}^{\prime}\right)$-module. The extended twisted Yangian $\mathrm{X}\left(\mathfrak{g}^{\prime}\right)$ acts on $\mathrm{M}_{\mu}^{\lambda}$ and hence on $\mathrm{L}_{\mu}^{\lambda}$ via the homomorphism $\mathrm{X}\left(\mathfrak{g}^{\prime}\right) \rightarrow \mathrm{A}^{\mathrm{G}}$. In Subsection 4.6 we derive the same irreducibilty properties of the $\mathrm{X}\left(\mathfrak{g}^{\prime}\right)$-module $\mathrm{L}_{\mu}^{\lambda}$, as described 
above for the image of our operator (4.58). Suppose that $\lambda+\rho$ is dominant. If $\mathrm{G}=\mathrm{Sp}_{2 m}$, then $\mathrm{L}_{\mu}^{\lambda}$ is an irreducible $\mathrm{X}\left(\mathfrak{g}^{\prime}\right)$-module for both $\mathfrak{g}^{\prime}=\mathfrak{s o}_{n}$ and $\mathfrak{g}^{\prime}=\mathfrak{s} \mathfrak{p}_{n}$. If $\mathrm{G}=\mathrm{O}_{2 m}$, then the $\mathrm{X}\left(\mathfrak{g}^{\prime}\right)$-module $\mathrm{L}_{\mu}^{\lambda}$ is irreducible under the extra condition that the stabilizer of the weight $\lambda+\rho$ in the extended Weyl group of $\mathfrak{g}=\mathfrak{s o}_{2 m}$ is contained in the proper Weyl group. If $\mathrm{G}=\mathrm{O}_{2 m}$ and $\theta=-1$, then $\mathrm{L}_{\mu}^{\lambda}$ is irreducible under the joint action of $\mathrm{X}\left(\mathfrak{s o}_{n}\right)$ and $\mathrm{O}_{n}$. Moreover, if $n$ is odd, then $\mathrm{L}_{\mu}^{\lambda}$ the image is irreducible under the action of the algebra $\mathrm{X}\left(\mathfrak{s o}_{n}\right)$ alone. But if $n$ is even, then $\mathrm{L}_{\mu}^{\lambda}$ is either an irreducible $\mathrm{X}\left(\mathfrak{s o}_{n}\right)$-module, or is a direct sum of two nonequivalent irreducible $\mathrm{X}\left(\mathfrak{s o}_{n}\right)$-modules .

Let $\left(\mathrm{G}, \mathrm{G}^{\prime}\right)$ once again be any pair from (0.1) other than $\left(\mathrm{GL}_{m}, \mathrm{GL}_{n}\right)$. Let $\lambda+\rho$ be dominant. When $\theta=-1$ or $n=1$, the conditions of Proposition 2.9 are satisfied if for each positive root $\alpha$ of $\mathfrak{g}$ the number $z_{\alpha}$, defined just before stating Proposition 4.6, is not zero. When $\theta=1$ and $n>1$, the conditions of Proposition 2.9 are satisfied if $z_{\alpha}$ is not zero for each compact positive root $\alpha$ of $\mathfrak{g}$. Under these conditions, the image of our intertwining operator (4.58) is not zero, and is equivalent to $\mathrm{L}_{\mu}^{\lambda}$ as $\mathrm{X}\left(\mathfrak{g}^{\prime}\right)$-module. Moreover, then the image is equivalent to $\mathrm{L}_{\mu}^{\lambda}$ under the joint actions of $\mathrm{X}\left(\mathfrak{g}^{\prime}\right)$ and $\mathrm{G}^{\prime}$.

In Subsection 4.7 we once again consider the $Y\left(\mathfrak{g}^{\prime}\right)$-intertwining operators (4.49) and (4.58) for $\mathfrak{g}^{\prime}=\mathfrak{g l}_{n}$ and $\mathfrak{g}^{\prime}=\mathfrak{s p}_{n}, \mathfrak{s o}_{n}$, respectively. We show that the target $\mathrm{Y}\left(\mathfrak{g}^{\prime}\right)$-modules of these operators are dual to the source modules. We show how this duality arises from the theory of Mickelsson algebras, when $\lambda+\rho$ is dominant.

0.5. Overview of Section 5. In Section 5 we use our intertwining operators (4.49) and (4.58) to give realizations of irreducible representations of the Yangian $\mathrm{Y}\left(\mathfrak{g l}_{n}\right)$ and of the twisted Yangians $\mathrm{Y}\left(\mathfrak{s p}_{n}\right), \mathrm{Y}\left(\mathfrak{s o}_{n}\right)$. We call two $\mathrm{Y}\left(\mathfrak{g l}_{n}\right)$-modules similar if they differ only by an automorphism (4.7) of $\mathrm{Y}\left(\mathfrak{g l}_{n}\right)$, where $g(x)$ is any formal power series in $x^{-1}$ with coefficients from $\mathbb{C}$ and leading term 1 . Up to equivalence and similarity, the irreducible finite-dimensional $\mathrm{Y}\left(\mathfrak{g l}_{n}\right)$-modules were classified by Drinfeld [D2, who generalized the classification given for $n=2$ by Tarasov [T1, T2]. It was then proved by Akasaka and Kashiwara AK that any of these modules can be realized as a quotient of a tensor product of $\mathrm{Y}\left(\mathfrak{g l}_{n}\right)$-modules of the form $\Phi_{t}^{k}$ with $\theta=-1$; see also the work of Chari and Pressley [CP. Further results were obtained by Chari [C] and by Brundan and Kleshchev [BK]. Note that the works [AK] and [C] deal with representations of quantum affine algebras. For a connection to the representation theory of Yangians, see the work of Molev, Tolstoy and Zhang [MTZ].

In Subsection 5.1 we give new proofs of the results from [AK for $\mathrm{Y}\left(\mathfrak{g l}_{n}\right)$. Namely, we prove that up to equivalence and similarity, any irreducible finite-dimensional $\mathrm{Y}\left(\mathfrak{g l}_{n}\right)$-module arises as the quotient by the kernel of intertwining operator (4.49) for $\theta=-1$, some $m$ and certain weights $\lambda, \mu$ of $\mathfrak{g l}_{m}$. Here the weight $\lambda+\rho$ is dominant, that is, it satisfies the conditions (5.1). The difference $\nu=\lambda-\mu$ satisfies the conditions (5.2), which come from Proposition 4.4. This proposition was obtained in KN1, KN2 and gives an explicit formula for the image under the operator (4.49) of a certain distinguished vector, called highest. For $\theta=-1$, the conditions (5.2) guarantee that the image is not zero; see Theorem 5.1,

Now take the twisted Yangians $\mathrm{Y}\left(\mathfrak{g}^{\prime}\right)$ where $\mathfrak{g}^{\prime}=\mathfrak{s p}_{n}, \mathfrak{s o}_{n}$. Any automorphism (4.7) of $\mathrm{Y}\left(\mathfrak{g l}_{n}\right)$ determines an automorphism of the subalgebra $\mathrm{Y}\left(\mathfrak{g}^{\prime}\right) \subset \mathrm{Y}\left(\mathfrak{g l}_{n}\right)$. We call two $\mathrm{Y}\left(\mathfrak{g}^{\prime}\right)$-modules similar if they differ only by such an automorphism. Up to equivalence and similarity, the irreducible finite-dimensional $Y\left(\mathfrak{g}^{\prime}\right)$-modules 
have been classified by Molev; see M2 for an exposition of the classification. No explicit realization of these $\mathrm{Y}\left(\mathfrak{g}^{\prime}\right)$-modules had been known so far in general. It is provided by our results. Thus we extend the works of Molev [M1] and the second named author of this article $[\mathbb{N}]$, which give explicit realizations of irreducible $\mathrm{Y}\left(\mathfrak{g}^{\prime}\right)$ modules from a particular class.

In Subsection 5.3 we prove that up to equivalence and similarity, any irreducible finite-dimensional $\mathrm{Y}\left(\mathfrak{s p}_{n}\right)$-module arises as quotient by the kernel of the operator (4.58) for $\theta=-1$, some $m$ and certain weights $\lambda, \mu$ of $\mathfrak{s p}_{2 m}$. Here the weight $\lambda+\rho$ is dominant, that is, it satisfies the conditions (5.9) -(5.11). The weight $\nu=\lambda-\mu-\kappa$ of $\mathfrak{s p}_{2 m}$ satisfies the conditions (5.12)-(5.14); see Theorem 5.3.

Recall that the twisted Yangian $\mathrm{Y}\left(\mathfrak{s o}_{n}\right)$ contains $\mathrm{U}\left(\mathfrak{s o}_{n}\right)$ as a subalgebra. We shall call a finite-dimensional module over the algebra $\mathrm{Y}\left(\mathfrak{s o}_{n}\right)$ an $\left(\mathrm{Y}\left(\mathfrak{s o}_{n}\right), \mathrm{O}_{n}\right)$ module if the group $\mathrm{O}_{n}$ also acts on this module, and the corresponding action of the Lie algebra $\mathfrak{s o}_{n}$ of $\mathrm{O}_{n}$ on this module coincides with the action obtained by restricting the action of $\mathrm{Y}\left(\mathfrak{s o}_{n}\right)$ to the subalgebra $\mathrm{U}\left(\mathfrak{s o}_{n}\right)$. If $n$ is odd, then any irreducible $\left(\mathrm{Y}\left(\mathfrak{s o}_{n}\right), \mathrm{O}_{n}\right)$-module is also irreducible over $\mathrm{Y}\left(\mathfrak{s o}_{n}\right)$. But if $n$ is even, then any $\left(\mathrm{Y}\left(\mathfrak{s o}_{n}\right), \mathrm{O}_{n}\right)$-module is either irreducible over $\mathrm{Y}\left(\mathfrak{s o}_{n}\right)$ or splits into a direct sum of two irreducible $\mathrm{Y}\left(\mathfrak{s o}_{n}\right)$-modules, which are not equivalent to each other. The irreducible $\mathrm{Y}\left(\mathfrak{s o}_{n}\right)$-modules occuring in this way for any $n$ are all those whose restriction to the subalgebra $\mathrm{U}\left(\mathfrak{s o}_{n}\right) \subset \mathrm{Y}\left(\mathfrak{s o}_{n}\right)$ integrates to a module of the special orthogonal group $\mathrm{SO}_{n} \subset \mathrm{O}_{n}$.

In Subsections 5.4 and 5.5 we prove that up to equivalence and similarity, any finite-dimensional irreducible $\left(\mathrm{Y}\left(\mathfrak{s o}_{n}\right), \mathrm{O}_{n}\right)$-module arises as the quotient by the kernel of the intertwining operator (4.58) for $\theta=-1$, some $m$ and certain weights $\lambda, \mu$ of $\mathfrak{s o}_{2 m}$. Here $\lambda+\rho$ is dominant, that is, it satisfies the conditions (5.16), (5.17). The weight $\nu=\lambda-\mu-\kappa$ of $\mathfrak{s o}_{2 m}$ satisfies (5.18), (5.19); see Theorems 5.4 and 5.5 .

Here for both $\mathfrak{g}^{\prime}=\mathfrak{s p}_{n}, \mathfrak{s o}_{n}$ the weight $\kappa$ of $\mathfrak{g}=\mathfrak{s p}_{2 m}, \mathfrak{s o}_{2 m}$, respectively, is defined by (2.12). The above-mentioned conditions on the weight $\nu$ of $\mathfrak{g}$ come from Proposition 4.6. This proposition was obtained in KN3, KN4 and gives an explicit formula for the image under the operator (4.58) of a certain distinguished vector, again called highest. For $\theta=-1$ our conditions on $\nu$ guarantee that the image is not zero. The notion of a highest vector relative to $\mathrm{Y}\left(\mathfrak{g}^{\prime}\right)$ for $\mathfrak{g}^{\prime}=\mathfrak{s p}_{n}, \mathfrak{s o}_{n}$ is discussed in Subsection 5.2.

\section{MickelsSon ALGEBRAS}

1.1. Generalities. Let $\mathfrak{g}$ be a reductive complex Lie algebra of semisimple rank $r$. Choose a triangular decomposition

$$
\mathfrak{g}=\mathfrak{n} \oplus \mathfrak{t} \oplus \mathfrak{n}^{\prime},
$$

where $\mathfrak{t}$ is a Cartan subalgebra, while $\mathfrak{n}$ and $\mathfrak{n}^{\prime}$ are the nilpotent radicals of two opposite Borel subalgebras of $\mathfrak{g}$ containing $\mathfrak{t}$. Consider the root system of $\mathfrak{g}$ in $\mathfrak{t}^{*}$. The set of the positive roots of $\mathfrak{g}$ will be denoted by $\Delta^{+}$.

Let $\alpha_{1}, \ldots, \alpha_{r} \in \Delta^{+}$be simple roots. For each $c=1, \ldots, r$, let $H_{c}=\alpha_{c}^{\vee} \in \mathfrak{t}$ be the coroot corresponding to the simple root $\alpha_{c}$. Let $E_{c} \in \mathfrak{n}^{\prime}$ and $F_{c} \in \mathfrak{n}$ be the root vectors corresponding to the roots $\alpha_{c}$ and $-\alpha_{c}$. We suppose that $\left[E_{c}, F_{c}\right]=H_{c}$. Let $\varepsilon$ be a Chevalley anti-involution on $\mathfrak{g}$. This is an involutive anti-automorphism 
of $\mathfrak{g}$ identical on $\mathfrak{t}$, such that for every $c=1, \ldots, r$, one has $\varepsilon\left(E_{c}\right)=\varepsilon_{c} F_{c}$ and $\varepsilon\left(F_{c}\right)=\varepsilon_{c}^{-1} E_{c}$ for some nonzero $\varepsilon_{c} \in \mathbb{C}$.

Let $\mathfrak{S}$ be the Weyl group of the root system of $\mathfrak{g}$. Let $\sigma_{1}, \ldots, \sigma_{r} \in \mathfrak{S}$ be the reflections in $\mathfrak{t}^{*}$ corresponding to the simple roots $\alpha_{1}, \ldots, \alpha_{r}$. We also use the induced action of the Weyl group $\mathfrak{S}$ on the vector space $\mathfrak{t}$. It is defined by setting $\lambda(\sigma(H))=\sigma^{-1}(\lambda)(H)$ for all $\sigma \in \mathfrak{S}, H \in \mathfrak{t}$ and $\lambda \in \mathfrak{t}^{*}$. Let $\rho \in \mathfrak{t}^{*}$ be the half-sum of the positive roots. Then the shifted action $\circ$ of the group $\mathfrak{S}$ on the vector space $t^{*}$ is defined by setting

$$
\sigma \circ \lambda=\sigma(\lambda+\rho)-\rho
$$

It induces an action $\circ$ of $\mathfrak{S}$ on the commutative algebra $\mathrm{U}(\mathfrak{t})$, by regarding the elements of this algebra as polynomial functions on $\mathfrak{t}^{*}$. In particular, for $H \in \mathfrak{t}$ we have $(\sigma \circ H)(\lambda)=H\left(\sigma^{-1} \circ \lambda\right)$. For any left $\mathfrak{g}$-module $K$ and any $\lambda \in \mathfrak{t}^{*}$, we will denote by $K^{\lambda}$ the subspace of weight $\lambda$ in $K$; we have $v \in K^{\lambda}$ if and only if $H v=\lambda(H) v$ for every $H \in \mathfrak{t}$.

Let $\mathrm{A}$ be any complex associative algebra, containing the universal enveloping algebra $\mathrm{U}(\mathfrak{g})$ as a subalgebra. Suppose there is also a vector subspace $\mathrm{V} \subset \mathrm{A}$, invariant under the adjoint action of the Lie algebra $\mathfrak{g}$ on A, such that:

(a) the multiplication map $\mathrm{U}(\mathfrak{g}) \otimes \mathrm{V} \rightarrow \mathrm{A}: X \otimes Y \mapsto X Y$ is bijective;

(b) the multiplication map $\mathrm{V} \otimes \mathrm{U}(\mathfrak{g}) \rightarrow \mathrm{A}: Y \otimes X \mapsto Y X$ is bijective;

(c) the space $\mathrm{V}$ is locally finite relative to the adjoint action of $\mathfrak{g}$.

Since the subspace $\mathrm{V} \subset \mathrm{A}$ is invariant under the adjoint action of $\mathfrak{g}$, the conditions (a) and (b) are equivalent. The condition (c) means that $\mathrm{V}$ can be decomposed into a direct sum of irreducible finite-dimensional $\mathfrak{g}$-modules. Since the adjoint action of $\mathfrak{g}$ on $U(\mathfrak{g})$ is locally finite, by (c) the same is true for the adjoint action of $\mathfrak{g}$ on A.

Let $\mathrm{J}$ be the right ideal generated by the elements of the subalgebra $\mathfrak{n} \subset \mathfrak{g}$. Let Norm $\mathrm{J} \subset \mathrm{A}$ be the normalizer of this right ideal, so that $Y \in$ Norm J if and only if $Y \mathrm{~J} \subset \mathrm{J}$. Then $\mathrm{J}$ is a two-sided ideal of Norm $\mathrm{J}$. The quotient algebra

$$
\mathrm{R}=\operatorname{Norm} \mathrm{J} / \mathrm{J}
$$

is called a Mickelsson algebra. By definition, the algebra $\mathrm{R}$ acts on the space $K / \mathfrak{n} K$ of $\mathfrak{n}$-coinvariants of any left A-module $K$. This space itself will be denoted by $K_{\mathfrak{n}}$.

Recall that $\mathrm{U}(\mathfrak{g}) \subset \mathrm{A}$. Hence we have $\mathrm{U}(\mathfrak{t}) \subset$ Norm J. It follows that the Mickelsson algebra $\mathrm{R}$ also contains $\mathrm{U}(\mathfrak{t})$ as subalgebra. Due to the condition (a) above, $\mathrm{R}$ is a torsion free module over $\mathrm{U}(\mathfrak{t})$ relative to the left multiplication. The condition (b) implies that $\mathrm{R}$ is a torsion free module over $\mathrm{U}(\mathfrak{t})$ relative to the right multiplication.

For each root $\alpha \in \mathfrak{t}^{*}$ let $H_{\alpha}=\alpha^{\vee} \in \mathfrak{t}$ be the corresponding coroot. The Weyl group $\mathfrak{S}$ acts on the vector space $\mathfrak{t}$ so that $\sigma: H_{\alpha} \mapsto H_{\sigma(\alpha)}$ for any $\sigma \in \mathfrak{S}$. Denote by $\overline{U(\mathfrak{t})}$ the ring of fractions of the commutative algebra $U(\mathfrak{t})$ relative to the set of denominators

$$
\left\{H_{\alpha}+z \mid \alpha \in \Delta^{+}, z \in \mathbb{Z}\right\} .
$$

The elements of $\overline{U(\mathfrak{t})}$ can be regarded as rational functions on the vector space $\mathfrak{t}^{*}$. The elements of $U(\mathfrak{t}) \subset \overline{U(\mathfrak{t})}$ are then regarded as polynomial functions on $\mathfrak{t}^{*}$. Let $\overline{\mathrm{U}(\mathfrak{g})}$ be the ring of fractions of the algebra $\mathrm{U}(\mathfrak{g})$ relative to the set of denominators (1.3). This ring is defined because $\mathrm{U}(\mathfrak{g})$ satisfies the Ore condition relative to (1.3). 
Now regard (1.3) as a subset of A using the embedding of $\mathfrak{t} \subset \mathfrak{g}$. Due to the conditions (a)-(c), the algebra A also satisfies the Ore condition relative to its subset (1.3). Let $\bar{A}$ be the ring of fractions of $A$ relative to the same set of denominators (1.3). Then $\bar{A}$ is a free left and free right $\overline{\mathrm{U}(\mathfrak{t})}$-module, which contains $\mathrm{A}$ as a subalgebra.

Denote by $\overline{\mathrm{J}}$ the right ideal of the algebra $\overline{\mathrm{A}}$ generated by the elements of $\mathfrak{n}$. Consider the normalizer Norm $\overline{\mathrm{J}} \subset \mathrm{A}$ of this right ideal. This normalizer coincides with the ring of fractions of Norm $J$ relative to the same set of denominators (1.3) as before. Now $\overline{\mathrm{J}}$ is a two-sided ideal of Norm $\overline{\mathrm{J}}$. The quotient algebra

$$
\overline{\mathrm{R}}=\operatorname{Norm} \overline{\mathrm{J}} / \overline{\mathrm{J}}
$$

bears the same name of Mickelsson algebra, as the quotient algebra $\mathrm{R}$ does. Note that $\overline{\mathrm{J}} \cap \mathrm{A}=\mathrm{J}$ because the subalgebra $\mathfrak{t} \subset \mathfrak{g}$ normalizes the subalgebra $\mathfrak{n} \subset \mathfrak{g}$. Hence the natural embedding $\mathrm{A} \rightarrow \overline{\mathrm{A}}$ determines an embedding $\mathrm{R} \rightarrow \overline{\mathrm{R}}$.

1.2. Double coset algebra. For each positive root $\alpha \in \Delta^{+}$, let $E_{\alpha} \in \mathfrak{n}^{\prime}$, and let $F_{\alpha} \in \mathfrak{n}$ be the corresponding root vectors. In particular, for any simple root $\alpha=\alpha_{c}$, we have $E_{\alpha}=E_{c}$ and $F_{\alpha}=F_{c}$. For any weight $\lambda \in \mathfrak{t}^{*}$ consider the infinite sum

$$
P_{\alpha}[\lambda]=1+\sum_{s=1}^{\infty}(-1)^{s}\left(s !\left(H_{\alpha}+\lambda\left(H_{\alpha}\right)+1\right) \cdots\left(H_{\alpha}+\lambda\left(H_{\alpha}\right)+s\right)\right)^{-1} F_{\alpha}^{s} E_{\alpha}^{s},
$$

where the denominators do not belong to the set (1.3) in general. The sum belongs to a certain algebra which contains $\overline{\mathrm{U}(\mathfrak{g})}$ as a subalgebra and does not need to be defined here; see [Z2, Section 1] for its definition. Take any $\sigma \in \mathfrak{S}$ and any reduced decomposition $\sigma=\sigma_{d_{l}} \cdots \sigma_{d_{1}}$. Here $l$ is the length of $\sigma$. Any such decomposition defines a normally ordered sequence of positive roots:

$$
\beta_{1}=\alpha_{d_{1}}, \quad \beta_{2}=\sigma_{d_{1}}\left(\alpha_{d_{2}}\right), \quad \ldots, \quad \beta_{l}=\sigma_{d_{1}} \sigma_{d_{2}} \cdots \sigma_{d_{l-1}}\left(\alpha_{d_{l}}\right) .
$$

The product

$$
P_{\sigma}[\lambda]=P_{\beta_{l}}[\lambda] \cdots P_{\beta_{1}}[\lambda]
$$

in the above-mentioned algebra does not depend on the choice of a decomposition; see [Z2, Section 2]. Let $\sigma_{0}$ be the longest element of the Weyl group $\mathfrak{S}$. Denote

$$
P[\lambda]=P_{\sigma_{0}}[\lambda] .
$$

The element $P=P[\lambda]$ with $\lambda=\rho$ is the extremal projector for the reductive Lie algebra $\mathfrak{g}$; its definition is due to Asherova, Smirnov and Tolstoy [AST. The element $P$ can be presented as an infinite sum of elements of the algebra $\overline{\mathrm{U}(\mathfrak{g})}$. Thus it belongs to a certain completion $\widetilde{U(\mathfrak{g})}$ of the latter algebra; the completion does not need to be defined here. Below are the basic properties of the extremal projector:

$$
\begin{gathered}
P^{2}=P ; \\
E_{\alpha} P=0 \text { and } P F_{\alpha}=0 \text { for } \alpha \in \Delta^{+} ; \\
P \in 1+\mathfrak{n} \widetilde{U(g)} \text { and } P \in 1+\widetilde{\mathrm{U}(\mathfrak{g})} \mathfrak{n}^{\prime} .
\end{gathered}
$$

For the proofs of (1.5) and (1.6), see [AST and [22, Section 3]. The two properties (1.7) follow directly from the definition (1.4), which also implies that $\varepsilon(P)=P$. 
Moreover,

$$
P \in 1+\sum_{s=1}^{\infty} \mathfrak{n}^{s} \overline{\mathrm{U}(\mathfrak{g})} \quad \text { and } \quad P \in 1+\sum_{s=1}^{\infty} \overline{\mathrm{U}(\mathfrak{g})}\left(\mathfrak{n}^{\prime}\right)^{s} .
$$

Now let $\mathrm{J}^{\prime}$ be the left ideal of A generated by elements of $\mathfrak{n}^{\prime}$. Similarly, let $\bar{J}^{\prime}$ be the left ideal of $\bar{A}$ generated by elements of $\mathfrak{n}^{\prime}$. First consider the double coset vector space

$$
\mathrm{Z}=\mathrm{A} /\left(\mathrm{J}+\mathrm{J}^{\prime}\right)
$$

where the quotient is taken by a left and a right ideal. Since the Cartan subalgebra $\mathfrak{t} \subset \mathfrak{g}$ normalizes the subalgebras $\mathfrak{n}$ and $\mathfrak{n}^{\prime}$, the left and right multiplications in A by the elements of the algebra $U(\mathfrak{t})$ make the vector space $\mathrm{Z}$ a $\mathrm{U}(\mathfrak{t})$-bimodule. Relative to the adjoint action of $\mathfrak{t}$ on $\mathrm{Z}$, we have a weight decomposition

$$
\mathrm{Z}=\underset{\lambda \in \mathrm{t}^{*}}{\oplus} \mathrm{Z}^{\lambda}
$$

Next consider the double coset vector space

$$
\overline{\mathrm{Z}}=\overline{\mathrm{A}} /\left(\overline{\mathrm{J}}+\overline{\mathrm{J}}^{\prime}\right) .
$$

This vector space is a bimodule over the algebra $\overline{\mathrm{U}(\mathfrak{t})}$. Since $\left(\overline{\mathrm{J}}+\overline{\mathrm{J}}^{\prime}\right) \cap \mathrm{A}=\mathrm{J}+\mathrm{J}^{\prime}$, the natural embedding $\mathrm{A} \rightarrow \overline{\mathrm{A}}$ determines an embedding of $\mathrm{U}(\mathfrak{t})$-bimodules $\mathrm{Z} \rightarrow \overline{\mathrm{Z}}$. Let us now equip the vector space $\overline{\mathrm{Z}}$ with a binary operation $\diamond$ defined by setting

$$
X \diamond Y=X P Y \quad \text { for } \quad X, Y \in \overline{\mathrm{Z}} .
$$

We first define the coset $X P Y \in \overline{\mathrm{Z}}$ in the case when $X, Y \in \mathrm{Z}$ by using the relations (1.6). Although $P$ is an infinite sum of elements of $\overline{\mathrm{U}(\mathfrak{g})}$, the condition (c) implies that only finitely many summands of $X P Y$ are nonzero cosets in $\bar{Z}$ in this case. Here we use (1.8). This definition of $X P Y$ extends to any cosets $X, Y \in \overline{\mathrm{Z}}$ by $\overline{\mathrm{U}(\mathfrak{t})}$ linearity. Note that each summand of $P$ commutes with every element of the subalgebra $\overline{\mathrm{U}(\mathfrak{t})} \subset \overline{\mathrm{U}(\mathfrak{g})}$.

So $\bar{Z}$ becomes an associative algebra over $\mathbb{C}$. We call it the double coset algebra. The quotient space $\overline{\mathrm{A}} / \overline{\mathrm{J}}$ is a left $\overline{\mathrm{Z}}$-module relative to an action, also denoted by $\diamond$, defined by

$$
X \diamond Z=X P Z \text { for } X \in \overline{\mathrm{Z}} \text { and } Z \in \overline{\mathrm{A}} / \overline{\mathrm{J}} .
$$

Recall that $\mathrm{R} \subset \overline{\mathrm{R}} \subset \overline{\mathrm{A}} / \overline{\mathrm{J}}$. Now let $\pi: \overline{\mathrm{A}} / \overline{\mathrm{J}} \rightarrow \overline{\mathrm{Z}}$ be the canonical projection, so that

$$
\pi(A+\overline{\mathrm{J}})=A+\overline{\mathrm{J}}+\overline{\mathrm{J}}^{\prime} \quad \text { for } \quad A \in \overline{\mathrm{A}} .
$$

Proposition 1.1. (i) The restriction of $\pi$ to $\mathrm{R}$ is a monomorphism of algebras.

(ii) The restriction of $\pi$ to $\overline{\mathrm{R}}$ is an isomorphism of algebras $\overline{\mathrm{R}}$ and $\overline{\mathrm{Z}}$.

Proof. The properties (1.6) of $P$ imply that the assignment $X \rightarrow X P$ defines a map $\bar{Z} \rightarrow \overline{\mathrm{R}}$. Namely, the first of the properties (1.6) implies that the double coset $X \in \overline{\mathrm{Z}}$ gets mapped to a single coset in $\overline{\mathrm{A}} / \overline{\mathrm{J}}$. The second property implies that the latter coset belongs to $\overline{\mathrm{R}}$. Although $P$ is an infinite sum of elements of $\overline{\mathrm{U}(\mathfrak{g})}$, by (1.8) the condition (c) implies that only finitely many summands of $X P$ are nonzero cosets in $\overline{\mathrm{A}} / \overline{\mathrm{J}}$. 
Let us denote by $\pi^{*}$ this map from $\overline{\mathrm{Z}}$ to $\overline{\mathrm{R}}$. Further, denote by $\pi_{*}$ the restriction to $\overline{\mathrm{R}}$ of the projection map $\pi: \overline{\mathrm{A}} / \overline{\mathrm{J}} \rightarrow \overline{\mathrm{Z}}$. The first property in (1.7) implies the equality

$$
X P=X \quad \text { for any } \quad X \in \overline{\mathrm{R}} .
$$

Hence the composition $\pi^{*} \pi_{*}$ is the identity map on $\overline{\mathrm{R}}$. The equality (1.11) also implies that $\pi_{*}$ is a homomorphism of algebras. Finally, the second property in (1.7) implies that the composition $\pi_{*} \pi^{*}$ is the identity map on $\bar{Z}$. Thus we get (ii), and hence (i).

Thus the algebra $\bar{Z}$ contains as a subalgebra a copy $\pi(\mathrm{R})$ of the algebra $\mathrm{R}$. It also contains as a subalgebra a copy of another Mickelsson algebra, defined as a quotient of normalizer of the left ideal $\mathrm{J}^{\prime} \subset \mathrm{A}$ relative to this ideal. However, the latter quotient algebra will not be used in this article. Note that both algebras $\overline{\mathrm{R}}$ and $\overline{\mathrm{Z}}$ act on the quotient vector space $\overline{\mathrm{A}} / \overline{\mathrm{J}}$. Here $\overline{\mathrm{R}}$ acts via the left multiplication in the algebra $\bar{A}$, while the action of $\bar{Z}$ is defined by (1.10). The proof of Proposition 1.1 also demonstrates that the isomorphism $\pi: \overline{\mathrm{R}} \rightarrow \overline{\mathrm{Z}}$ identifies these two actions.

The algebra $\bar{Z}$ contains $\overline{U(\mathfrak{t})}$ as a subalgebra and is free both as a left and as a right module over it. Further, there is a weight decomposition of $\bar{Z}$ relative to the adjoint action of $\mathfrak{t}$ on $\bar{Z}$, similar to (1.9). The zero weight component $\bar{Z}^{0}$ is a subalgebra of $\bar{Z}$. The algebra $\bar{Z}^{0}$ has several natural subalgebras. One of them is the image $\pi\left(\mathrm{R}^{0}\right)$ of the zero weight component of the algebra $R$. Further, consider the image of the zero weight component $\mathrm{A}^{0}$ of $\mathrm{A} \subset \overline{\mathrm{A}}$ relative to the canonical projection $\overline{\mathrm{A}} \rightarrow \overline{\mathrm{Z}}$. This image is a vector subspace in $\bar{Z}$. Denote by $S$ the subalgebra in $\bar{Z}$ generated by the elements of this subspace. Then

$$
\pi\left(\mathrm{R}^{0}\right) \subset \mathrm{S} \subset \overline{\mathrm{Z}}^{0} .
$$

1.3. Algebraic group action. Let $\mathrm{G}$ be a reductive algebraic group over $\mathbb{C}$ with the Lie algebra $\mathfrak{g}$. Suppose there is an action of the group $G$ by automorphisms on the algebra A, extending the adjoint action of $G$ on the subalgebra $U(\mathfrak{g}) \subset A$. Suppose that the action of $\mathrm{G}$ on $\mathrm{A}$ preserves the subspace $\mathrm{V}$, and that the action of $\mathrm{G}$ on $\mathrm{V}$ is locally finite. Moreover, suppose that the action of $\mathfrak{g}$ on A corresponding to that of $\mathrm{G}$ coincides with the adjoint action:

$$
X: A \mapsto \operatorname{ad}_{X}(A)=X A-A X \quad \text { for } \quad X \in \mathfrak{g} \text { and } A \in \mathrm{A} .
$$

Let $\mathrm{G}_{0}$ be the connected component of $\mathrm{G}$ and $\mathrm{T} \subset \mathrm{G}_{0}$ be the maximal torus of $\mathrm{G}$ with the Lie algebra $\mathfrak{t}$. Let Norm $\mathrm{T}$ and $\mathrm{Norm}_{0} \mathrm{~T}$ be the normalizers of $\mathrm{T}$ in $\mathrm{G}$ and $G_{0}$, respectively. The adjoint action of the group Norm $_{0} \mathrm{~T}$ on $\mathfrak{t}$ identifies the quotient group $\operatorname{Norm}_{0} \mathrm{~T} / \mathrm{T}$ with the Weyl group $\mathfrak{S}$ of $\mathfrak{g}$. Choose a representative $\widehat{\sigma}_{c} \in \mathrm{Norm}_{0} \mathrm{~T}$ of $\sigma_{c}$. The elements $\widehat{\sigma}_{1}, \ldots, \widehat{\sigma}_{r} \in \mathrm{G}$ can be chosen to satisfy the braid relations

$$
\underbrace{\widehat{\sigma}_{c} \widehat{\sigma}_{d} \widehat{\sigma}_{c} \cdots}_{m_{c d}}=\underbrace{\widehat{\sigma}_{d} \widehat{\sigma}_{c} \widehat{\sigma}_{d} \cdots}_{m_{c d}} \text { for } c \neq d
$$

where $m_{c d}$ is the order of the element $\sigma_{c} \sigma_{d} \in \mathfrak{S}$; see for instance [T. For any reduced decomposition $\sigma=\sigma_{c_{1}} \cdots \sigma_{c_{k}}$ in $\mathfrak{S}$ put

$$
\widehat{\sigma}=\widehat{\sigma}_{c_{1}} \cdots \widehat{\sigma}_{c_{k}} .
$$

By (1.13) this definition of $\widehat{\sigma}$ is independent of the choice of the decomposition of $\sigma$. So we get an action of the braid group of $\mathfrak{g}$ by automorphisms $\widehat{\sigma}$ of the algebra A. 
The adjoint action of the group Norm $\mathrm{T}$ on $\mathfrak{t}$ identifies the quotient Norm $\mathrm{T} / \mathrm{T}$ with a semidirect product $\mathfrak{R}=\mathfrak{T} \ltimes \mathfrak{S}$ where $\mathfrak{T}$ is a certain finite subgroup of Norm $\mathrm{T} / \mathrm{T}$. Each element $\tau \in \mathfrak{T}$ acts on $\mathfrak{t}$ by permuting $H_{1}, \ldots, H_{r}$. Hence $\tau$ defines an automorphism of the semisimple part of $\mathfrak{g}$, which permutes $E_{1}, \ldots, E_{r}$ and in the same way permutes $F_{1}, \ldots, F_{r}$. For each element $\tau \in \mathfrak{T}$ choose a representative $\widehat{\tau} \in$ Norm $\mathrm{T}$ whose adjoint action on the semisimple part of $\mathfrak{g}$ yields this automorphism. Then $\widehat{\tau} \mapsto \tau$ and $\widehat{\sigma} \mapsto \sigma$ under the canonical map

$$
\text { Norm } \mathrm{T} \rightarrow \operatorname{Norm} \mathrm{T} / \mathrm{T}=\mathfrak{R} \text {. }
$$

Moreover, the representatives $\widehat{\sigma}_{1}, \ldots, \widehat{\sigma}_{r}$ above can be chosen so that the adjoint action of $\widehat{\tau}$ on $\mathrm{G}$ permutes them in the same way as $\tau$ permutes $H_{1}, \ldots, H_{r}$.

Regard $\mathfrak{g}$ as a subspace of $\mathrm{A}$. Note that because the action of $\widehat{\sigma} \in \mathrm{G}$ on the subspace $\mathfrak{t} \subset$ A factors through that of $\sigma \in \mathfrak{S}$, the action of $\widehat{\sigma}$ on A maps any vector of weight $\lambda$ to a vector of weight $\sigma(\lambda)$. Let $\mathrm{A}^{0} \subset \mathrm{A}$ be the zero weight subspace relative to the adjoint action of $\mathfrak{t}$. This subspace is preserved by the action of all the elements $\widehat{\sigma}, \widehat{\tau} \in \mathrm{G}$. Since the subgroup $\mathrm{T} \subset \mathrm{G}$ acts on $\mathrm{A}^{0}$ trivially, we get an action of the group $\mathfrak{R}$ on $\mathrm{A}^{0}$.

Since the action of the elements $\widehat{\sigma}, \widehat{\tau} \in \mathrm{G}$ on A preserves the set (1.3), it extends from A to $\overline{\mathrm{A}}$. Further, the action of $\widehat{\tau}$ on A preserves the ideals J and $\mathrm{J}^{\prime}$. Hence $\widehat{\tau}$ acts on A/J and on Z. Similarly, $\widehat{\tau}$ acts on $\bar{A}$ and on $\bar{Z}$.

1.4. Zhelobenko operators. For $c=1, \ldots, r$, define a linear map $\xi_{c}: \mathrm{A} \rightarrow \overline{\mathrm{A}}$ by setting for any $A \in \mathrm{A}$

$$
\xi_{c}(A)=A+\sum_{s=1}^{\infty}\left(s ! H_{c}\left(H_{c}-1\right) \cdots\left(H_{c}-s+1\right)\right)^{-1} E_{c}^{s} \operatorname{ad}_{F_{c}}^{s}(A) .
$$

By (c) for any given $A \in \mathrm{A}$ only finitely many terms of the sum (1.14) differ from zero, hence the map $\xi_{c}$ is well defined. The definition (1.14) and the next two propositions go back to [Z1, Section 2]. See [KN1, Section 3] for detailed proofs of these propositions.

Proposition 1.2. For any $H \in \mathfrak{t}$ and $A \in \mathrm{A}$, we have

$$
\begin{aligned}
& \xi_{c}(H A) \in\left(H+\alpha_{c}(H)\right) \xi_{c}(A)+\overline{\mathrm{J}}, \\
& \xi_{c}(A H) \in \xi_{c}(A)\left(H+\alpha_{c}(H)\right)+\overline{\mathrm{J}} .
\end{aligned}
$$

Proposition 1.2 allows us to define a linear map $\bar{\xi}_{c}: \overline{\mathrm{A}} \rightarrow \overline{\mathrm{A}} / \overline{\mathrm{J}}$ by setting

$$
\bar{\xi}_{c}(X A)=Z \xi_{c}(A)+\overline{\mathrm{J}} \quad \text { for } \quad X \in \overline{\mathrm{U}(\mathfrak{t})} \text { and } \quad A \in \mathrm{A},
$$

where the element $Z \in \overline{\mathrm{U}(\mathfrak{t})}$ is defined by the equality $Z(\mu)=X\left(\mu+\alpha_{c}\right)$ for $\mu \in \mathfrak{t}^{*}$ when $X$ and $Z$ are regarded as rational functions on $t^{*}$.

The action of the Weyl group $\mathfrak{S}$ on the algebra $U(\mathfrak{t})$ extends to an action of $\mathfrak{S}$ on $\overline{\mathrm{U}(\mathfrak{t})}$, so that for any $\sigma \in \mathfrak{S}$ we have

$$
(\sigma X)(\mu)=X\left(\sigma^{-1}(\mu)\right)
$$

if the element $X \in \overline{\mathrm{U}(\mathfrak{t})}$ is regarded as a rational function on $\mathfrak{t}^{*}$. Take the images $\widehat{\sigma}_{c}(\overline{\mathrm{J}})$ and $\widehat{\sigma}_{c}\left(\overline{\mathrm{J}}^{\prime}\right)$ relative to the action of $\widehat{\sigma}_{c} \in \mathrm{G}$ on $\overline{\mathrm{A}}$; they are, respectively, right and left ideals of $\bar{A}$.

Proposition 1.3. We have $\widehat{\sigma}_{c}(\overline{\mathrm{J}}) \subset \operatorname{Ker} \bar{\xi}_{c}$ and $\bar{\xi}_{c}\left(\widehat{\sigma}_{c}\left(\overline{\mathrm{J}}^{\prime}\right)\right) \subset \overline{\mathrm{J}}^{\prime}+\overline{\mathrm{J}}$. 
This proposition allows us to define the linear maps

$$
\check{\xi}_{c}: \overline{\mathrm{A}} / \overline{\mathrm{J}} \rightarrow \overline{\mathrm{A}} / \overline{\mathrm{J}} \text { and } \quad \check{\xi}_{c}: \overline{\mathrm{Z}} \rightarrow \overline{\mathrm{Z}}
$$

as the composition $\bar{\xi}_{c} \widehat{\sigma}_{c}$ applied to elements of $\overline{\mathrm{A}}$ which are taken modulo $\overline{\mathrm{J}}$ and $\overline{\mathrm{J}}+\overline{\mathrm{J}}^{\prime}$, respectively. In their present form, the maps $\check{\xi}_{c}$ have been introduced in KO]. The next proposition states their key properties. For the proof of part (i), see [Z1, Section 6]; for the proofs of (ii) and (iii), see [KO, Section 5].

Proposition 1.4. (i) The maps $\check{\xi}_{1}, \ldots, \check{\xi}_{r}$ of $\overline{\mathrm{A}} / \overline{\mathrm{J}}$ and $\overline{\mathrm{Z}}$ satisfy the braid relations

$$
\underbrace{\check{\xi}_{c} \check{\xi}_{d} \check{\xi}_{c} \cdots}_{m_{c d}}=\underbrace{\check{\xi}_{d} \check{\xi}_{c} \check{\xi}_{d} \cdots}_{m_{c d}} \text { for } c \neq d \text {. }
$$

(ii) The map $\check{\xi}_{c}$ of $\overline{\mathrm{Z}}$ is an automorphism of the double coset algebra:

$$
\check{\xi}_{c}(X \diamond Y)=\check{\xi}_{c}(X) \diamond \check{\xi}_{c}(Y) \text { for } X, Y \in \overline{\mathrm{Z}} .
$$

(iii) The map $\check{\xi}_{c}$ of $\overline{\mathrm{A}} / \overline{\mathrm{J}}$ is $\overline{\mathrm{Z}}$-equivariant in the following sense:

$$
\check{\xi}_{c}(X \diamond Z)=\check{\xi}_{c}(X) \diamond \check{\xi}_{c}(Z) \quad \text { for } \quad X \in \overline{\mathrm{Z}} \quad \text { and } \quad Z \in \overline{\mathrm{A}} / \overline{\mathrm{J}}
$$

Part (i) of Proposition 1.4 implies that for any reduced decomposition of an element $\sigma=\sigma_{c_{1}} \cdots \sigma_{c_{k}}$ in the Weyl group $\mathfrak{S}$, the map

$$
\check{\xi}_{\sigma}=\check{\xi}_{c_{1}} \ldots \check{\xi}_{c_{k}}
$$

of $\overline{\mathrm{A}} / \overline{\mathrm{J}}$ or $\overline{\mathrm{Z}}$ does not depend on the choice of the decomposition. In view of part (ii), any map $\check{\xi}_{\sigma}$ of $\overline{\mathrm{Z}}$ will be called a Zhelobenko automorphism of $\overline{\mathrm{Z}}$. Any map $\check{\xi}_{\sigma}$ of $\overline{\mathrm{A}} / \overline{\mathrm{J}}$ will be called a Zhelobenko operator on $\overline{\mathrm{A}} / \overline{\mathrm{J}}$. Then using the same symbol $\check{\xi}_{\sigma}$ for the maps of both $\overline{\mathrm{A}} / \overline{\mathrm{J}}$ and $\overline{\mathrm{Z}}$ should cause no confusion. By Proposition 1.2

$$
\begin{aligned}
& \check{\xi}_{\sigma}(H Z)=(\sigma \circ H) \check{\xi}_{\sigma}(Z), \\
& \check{\xi}_{\sigma}(Z H)=\check{\xi}_{\sigma}(Z)(\sigma \circ H)
\end{aligned}
$$

for any $\sigma \in \mathfrak{S}, H \in \mathfrak{t}$ and $Z \in \overline{\mathrm{A}} / \overline{\mathrm{J}}$ or $Z \in \overline{\mathrm{Z}}$. Here for $c=1, \ldots, r$ we use the equality

$$
\sigma_{c}\left(\mu+\alpha_{c}\right)=\sigma_{c} \circ \mu \text { for } \mu \in \mathfrak{t}^{*} .
$$

The relations (1.16) imply that all the operators $\check{\xi}_{\sigma}$ on $\bar{Z}$ preserve the subspace $\bar{Z}^{0}$.

We will complete this subsection with an explicit formula for the operator $\check{\xi}_{c}$ on $\bar{Z}$. Let us denote by $\mathfrak{g}_{c}$ the $\mathfrak{s l}_{2}$-subalgebra of $\mathfrak{g}$ spanned by the elements $E_{c}, F_{c}$ and $H_{c}$. It acts on the vector space $\mathrm{A}$, by restricting to $\mathfrak{g}_{c}$ the adjoint action of $\mathfrak{g}$ on $\mathrm{A}$. As a $\mathfrak{g}_{c}$-module, A splits into a direct sum of irreducible finite-dimensional submodules. Choose $j \in\left\{0, \frac{1}{2}, 1, \ldots\right\}$ and take any element $A \in \mathrm{A}$ from an irreducible $\mathfrak{g}_{c^{-}}$ submodule of dimension $2 j+1$. Suppose that $A$ is of weight $2 h$ relative to the action of $H_{c}$, that is, $\left[H_{c}, A\right]=2 h A$ for $h \in\{-j,-j+1, \ldots, j-1, j\}$. Since $\widehat{\sigma}_{c}$ is an automorphism of the algebra $\mathrm{A}$, the element $\widehat{\sigma}_{c}(A) \in \mathrm{A}$ belongs to an irreducible $\mathfrak{g}_{c}$-submodule of the same dimension $2 j+1$, and is of weight $-2 h$ relative to the action of $H_{c}$. Therefore the operator $\check{\xi}_{c}$ on $\bar{Z}$ maps the coset of $A$ to the coset of

$$
\begin{aligned}
& \sum_{s=0}^{\infty}\left(s ! H_{c}\left(H_{c}-1\right) \cdots\left(H_{c}-s+1\right)\right)^{-1} E_{c}^{s} \operatorname{ad}_{F_{c}}^{s}\left(\widehat{\sigma}_{c}(A)\right) \\
= & \sum_{s=0}^{j-h}\left(s ! H_{c}\left(H_{c}-1\right) \cdots\left(H_{c}-s+1\right)\right)^{-1} E_{c}^{s} \operatorname{ad}_{F_{c}}^{s}\left(\widehat{\sigma}_{c}(A)\right) .
\end{aligned}
$$


By the definition of subspace $\overline{\mathrm{J}} \subset \overline{\mathrm{A}}$, the coset of this sum coincides with that of

$$
\sum_{s=0}^{j-h}\left(s ! H_{c}\left(H_{c}-1\right) \cdots\left(H_{c}-s+1\right)\right)^{-1} \operatorname{ad}_{E_{c}}^{s} \operatorname{ad}_{F_{c}}^{s}\left(\widehat{\sigma}_{c}(A)\right) .
$$

But the coset of the last sum in $\bar{Z}$ coincides with the coset of

$$
\sum_{s=0}^{j-h} \prod_{i=0}^{s-1} \frac{(j-h-i)(j+h+i+1)}{(i+1)\left(H_{c}-i\right)} \widehat{\sigma}_{c}(A) ;
$$

see for instance the proof of [KN1, Proposition 3.7]. The sum over $s=0, \ldots, j-h$ of the products of fractions in (1.18) is a particular value $\mathrm{F}\left(h-j, j+h+1,-H_{c} ; 1\right)$ of the hypergeometric function. Using the well-known formula

$$
\mathrm{F}(u, v, w ; 1)=\frac{\Gamma(w) \Gamma(w-u-v)}{\Gamma(w-u) \Gamma(w-v)},
$$

valid for $u, v, w \in \mathbb{C}$ with $w \neq 0,-1, \ldots$ and $\operatorname{Re}(w-u-v)>0$, the sum (1.18) equals

$$
\begin{gathered}
\frac{\Gamma\left(-H_{c}\right) \Gamma\left(-H_{c}-2 h-1\right)}{\Gamma\left(-H_{c}-h+j\right) \Gamma\left(-H_{c}-h-j-1\right)} \widehat{\sigma}_{c}(A)=\prod_{i=1}^{j-h} \frac{H_{c}+2 h+i+1}{H_{c}-i+1} \widehat{\sigma}_{c}(A) \\
=\prod_{i=1}^{j-h}\left(H_{c}-i+1\right)^{-1} \cdot \widehat{\sigma}_{c}(A) \cdot \prod_{i=1}^{j-h}\left(H_{c}+i+1\right) .
\end{gathered}
$$

1.5. Invariants of Zhelobenko automorphisms. Consider the subspace $A^{\mathrm{G}}$ of $\mathrm{G}$-invariants in A. Define the linear map $\gamma: \mathrm{A}^{\mathrm{G}} \rightarrow \mathrm{Z}$ as the restriction to $\mathrm{A}^{\mathrm{G}}$ of the canonical projection $\mathrm{A} \rightarrow \mathrm{Z}$. The natural embedding of algebras $\mathrm{A} \rightarrow \overline{\mathrm{A}}$ determines an embedding of $\mathrm{U}(\mathfrak{t})$-bimodules $\mathrm{Z} \rightarrow \overline{\mathrm{Z}}$. Consider the zero weight component $\mathrm{Z}^{0}$ of the vector space $Z$. Here we again refer to the adjoint action of $t$ on $Z$. The definition of the action of $\check{\xi}_{\sigma}$ and $\widehat{\tau}$ on $\bar{Z}$ implies immediately that the image of the map $\gamma$ is contained in the subspace of $\mathrm{Z}$,

$$
\mathrm{Q}=\left\{Z \in \mathrm{Z}^{0} \mid \check{\xi}_{\sigma}(Z)=Z \text { and } \widehat{\tau}(Z)=Z \text { for all } \sigma \in \mathfrak{S} \text { and } \tau \in \mathfrak{T}\right\} .
$$

By [KNV] Remark 4] our Q is a subalgebra of $\bar{Z}$ and is included in the chain (1.12)

$$
\mathrm{Q} \subset \pi\left(\mathrm{R}^{0}\right) \subset \mathrm{S} \subset \overline{\mathrm{Z}}^{0} .
$$

The next proposition has been also proved in $\mathrm{KNV}$.

Proposition 1.5. The map $\gamma: \mathrm{A}^{\mathrm{G}} \rightarrow \mathrm{Z}$ is injective, and its image is equal to $\mathrm{Q}$.

The squares of the Zhelobenko automorphisms $\check{\xi}_{1}, \ldots, \check{\xi}_{r}$ of $\overline{\mathrm{Z}}$ are given by

$$
\check{\xi}_{c}^{2}(Z)=\left(H_{c}+1\right) \widehat{\sigma}_{c}^{2}(Z)\left(H_{c}+1\right)^{-1} \text { for all } Z \in \bar{Z} ;
$$

see [KO, Corollary 7.5]. Here $\widehat{\sigma}_{c}^{2} \in \mathrm{T}$ by definition, so that the squares $\widehat{\sigma}_{c}^{2}$ and hence $\check{\xi}_{c}^{2}$ act trivially on the zero weight subspace $\overline{\mathrm{Z}}^{0} \subset \overline{\mathrm{Z}}$. This means that the restrictions of all the operators $\check{\xi}_{\sigma}$ to $\overline{\mathrm{Z}}^{0}$ determine an action of the Weyl group $\mathfrak{S}$. Furthermore, for any $\tau \in \mathfrak{T}$ the adjoint action of $\widehat{\tau} \in \mathrm{G}$ on $\mathfrak{g}$ permutes the operators $\check{\xi}_{1}, \ldots, \check{\xi}_{r}$ on $\bar{Z}$ in the same way, as it permutes the elements $H_{1}, \ldots, H_{r}$ of $\mathfrak{t}$. This implies that the latter action of the group $\mathfrak{S}$ on $\bar{Z}^{0}$ extends to that of the semidirect product $\mathfrak{R}=\mathfrak{T} \ltimes \mathfrak{S}$.

We call a weight $\mu \in \mathfrak{t}^{*}$ dominant if $\mu\left(H_{\alpha}\right) \neq-1,-2, \ldots$ for all $\alpha \in \Delta^{+}$. Fix any $\lambda \in \mathfrak{t}^{*}$ such that $\lambda+\rho$ is dominant. Let $N$ be any irreducible $\mathrm{S}$-module of 
weight $\lambda$. The latter condition means that the subalgebra $\mathrm{U}(\mathfrak{t}) \subset \mathrm{S}$ acts on $N$ via the mapping $\lambda: \mathfrak{t} \rightarrow \mathbb{C}$. This mapping extends to a homomorphism $\mathrm{U}(\mathfrak{t}) \rightarrow \mathbb{C}$, also denoted by $\lambda$.

Due to (1.19), $N$ is a module over the algebra Q by restriction. Let $\mathfrak{S}_{\lambda}$ and $\mathfrak{R}_{\lambda}$ denote the stabilizers of $\lambda \in \mathfrak{t}^{*}$ in $\mathfrak{R}$ and $\mathfrak{S}$ relative to the shifted actions of these groups on $\mathfrak{t}^{*}$;

$$
\mathfrak{S}_{\lambda}=\{\sigma \in \mathfrak{S} \mid \sigma \circ \lambda=\lambda\}
$$

The shifted action of $\mathfrak{S}$ on $\mathfrak{t}^{*}$ extends to an action of $\mathfrak{R}$ since $\tau(\rho)=\rho$ for $\tau \in \mathfrak{T}$.

Proposition 1.6. Suppose that $\lambda+\rho \in \mathfrak{t}^{*}$ is dominant and that $\mathfrak{R}_{\lambda}=\mathfrak{S}_{\lambda}$. Then $N$ is an irreducible Q-module.

We shall prove this in the remainder of the subsection. Let $\chi: \mathrm{S} \rightarrow$ End $N$ be the defining homomorphism of the S-module $N$. For the proof of the proposition it is sufficient to find for each $Y \in \mathrm{S}$ an element $Z \in \mathrm{Q}$ such that $\chi(Y)=\chi(Z)$. Here we will assume that $Y$ is the image of some element $A \in \mathrm{A}^{0}$ under the canonical projection $\overline{\mathrm{A}} \rightarrow \overline{\mathrm{Z}}$; see the definition of the subalgebra $\mathrm{S}$ given at the end of Subsection 1.2.

Lemma 1.7. There exists $X \in \mathrm{U}(\mathfrak{t})$ such that $\lambda(X) \neq 0$, and for any $\sigma \in \mathfrak{S}$ the element $\check{\xi}_{\sigma}(X Y) \in \overline{\mathrm{Z}}$ belongs to the image of $\mathrm{A}^{0}$ under the canonical projection $\overline{\mathrm{A}} \rightarrow \overline{\mathrm{Z}}$.

Proof. All elements $\widehat{\sigma}(A)$ with $\sigma \in \mathfrak{S}$ belong to some finite-dimensional submodule $M$ of $\mathrm{A}^{0}$ under the adjoint action of $\mathfrak{g}$. For every positive root $\alpha \in \Delta^{+}$, we can choose a nonnegative integer $n_{\alpha}$ such that

$$
\operatorname{ad}_{F_{\alpha}}^{n_{\alpha}+1}(B)=0 \text { for all } B \in M \text {. }
$$

Put

$$
X=\prod_{\alpha \in \Delta^{+}} \prod_{s=1}^{n_{\alpha}}\left(H_{\alpha}+\rho\left(H_{\alpha}\right)+s\right) .
$$

Then we have $\lambda(X) \neq 0$, because the weight $\lambda+\rho$ is dominant by our assumption.

Now take any element $\sigma \in \mathfrak{S}$ and a reduced decomposition $\sigma=\sigma_{c_{1}} \cdots \sigma_{c_{k}}$. Put

$$
\omega_{l}=\sigma_{c_{1}} \cdots \sigma_{c_{l}} \text { and } \beta_{l}=\omega_{l-1}\left(\alpha_{c_{l}}\right) \text { for } l=1, \ldots, k .
$$

Note that $\beta_{1}, \ldots, \beta_{k}$ are all those roots $\alpha \in \Delta^{+}$for which $\sigma^{-1}(\alpha) \notin \Delta^{+}$. By (1.16),

$$
\check{\xi}_{\sigma}(X Y)=(\sigma \circ X) \check{\xi}_{\sigma}(Y) .
$$

In each factor $\check{\xi}_{c_{l}}$ of $\check{\xi}_{\sigma}$ in the product (1.15) we can replace, modulo the left ideal $\bar{J}^{\prime}$ of $\overline{\mathrm{A}}$, every entry of $E_{c_{l}}$ by the corresponding adjoint operator. Using the property (1.16) repeatedly, in $\check{\xi}_{\sigma}(Y)$ we can also move to the left all denominators from $\mathrm{U}(\mathfrak{t})$. Hence $\check{\xi}_{\sigma}(Y)$ equals the coset in $\overline{\mathrm{Z}}$ of the image of $\widehat{\sigma}(A)$ under the operator

$$
\sum_{s_{1}, \ldots, s_{k}=0}^{\infty} \prod_{l=1}^{k}\left(s_{l} !\left(\omega_{l-1} \circ H_{c_{l}}\right) \cdots\left(\omega_{l-1} \circ H_{c_{l}}-s_{l}+1\right)\right)^{-1} \cdot \operatorname{ad}_{E_{\beta_{1}}}^{s_{1}} \operatorname{ad}_{F_{\beta_{1}}}^{s_{1}} \cdots \operatorname{ad}_{E_{\beta_{k}}}^{s_{k}} \operatorname{ad}_{F_{\beta_{k}}}^{s_{k}} .
$$


By the assumption (1.20), all summands above with at least one index $s_{l}>n_{\beta_{l}}$ vanish. Further, for all $l=1, \ldots, k$ and $s=1,2, \ldots$ we have

$$
\begin{aligned}
\omega_{l-1} \circ H_{c_{l}}-s+1 & =H_{\beta_{l}}+\left(\omega_{l-1}^{-1}(\rho)-\rho\right)\left(H_{c_{l}}\right)-s+1 \\
& =H_{\beta_{l}}+\rho\left(H_{\beta_{l}}\right)-1-s+1 \\
& =H_{\beta_{l}}+\rho\left(H_{\beta_{l}}\right)-s .
\end{aligned}
$$

Now for $l=1, \ldots, k$ consider the factor in (1.21) corresponding to the positive root $\alpha=-\sigma^{-1}\left(\beta_{l}\right)$ and an index $s=1, \ldots, n_{\alpha}$. The shifted action of $\sigma$ on this factor yields

$\sigma \circ H_{\alpha}+\rho\left(H_{\alpha}\right)+s=-H_{\beta_{l}}+\left(\sigma^{-1}(\rho)-\rho\right)\left(H_{\alpha}\right)+\rho\left(H_{\alpha}\right)+s=-H_{\beta_{l}}-\rho\left(H_{\beta_{l}}\right)+s$.

The latter cancels, up to the minus sign, the factor (1.22) in the denominator of $\check{\xi}_{\sigma}(Y)$. Hence the product $(\sigma \circ X) \check{\xi}_{\sigma}(Y) \in \overline{\mathrm{Z}}$ belongs to the image of $\mathrm{A}^{0}$.

Take the element $X \in \mathrm{U}(\mathfrak{t})$ from Lemma 1.7. We have $\chi(X Y)=\lambda(X) \chi(Y)$ because the $\mathrm{S}$-module is of weight $\lambda$ by our assumption. Hence

$$
\chi(Y)=\chi(X Y / \lambda(X)) \text {. }
$$

Replacing the given element $Y \in \mathrm{S}$ by $X Y / \lambda(X)$, we may from now on assume that for any $\sigma \in \mathfrak{S}$ the element $\check{\xi}_{\sigma}(Y) \in \overline{\mathrm{Z}}$ belongs to the image of $\mathrm{A}^{0}$ under the projection $\overline{\mathrm{A}} \rightarrow \overline{\mathrm{Z}}$.

Consider the orbit of $\lambda$ relative to the shifted action of the group $\mathfrak{R}$ on $\mathfrak{t}^{*}$,

$$
\mathcal{O}_{\lambda}=\{\omega \circ \lambda \mid \omega \in \mathfrak{R}\} \text {. }
$$

This is a finite subset of $\mathfrak{t}^{*}$. Hence there exists an element $X^{\prime} \in \mathrm{U}(\mathfrak{t})$, such that $\lambda\left(X^{\prime}\right)=1$ and $\mu\left(X^{\prime}\right)=0$ for any weight $\mu \in \mathcal{O}_{\lambda}$ with $\mu \neq \lambda$. Put

$$
Z=\left|\mathfrak{S}_{\lambda}\right|^{-1} \sum_{\omega \in \mathfrak{R}} \check{\xi}_{\omega}\left(X^{\prime} Y\right) .
$$

Here $\check{\xi}_{\omega}$ denotes the composition $\widehat{\tau} \check{\xi}_{\sigma}$ of operators on $\bar{Z}$, if $\omega=\tau \sigma$ for some $\sigma \in \mathfrak{S}$ and $\tau \in \mathfrak{T}$. Due to our assumptions on the given element $Y$, we have $Z \in \mathrm{S}$. Moreover, for every $\omega \in \mathfrak{R}$, we have $\check{\xi}_{\omega}(Z)=Z$. Thus $Z \in \mathrm{Q}$. Let us show that $\chi(Y)=\chi(Z)$.

Until the end of this section, the symbol $\equiv$ will indicate equalities in the algebra $\overline{\mathrm{Z}}$ modulo the right ideal generated by all the elements $H-\lambda(H)$ with $H \in \mathfrak{t}$. First, take any $\omega \in \mathfrak{R}$ such that $\omega \circ \lambda \neq \lambda$. By our choice of the element $X^{\prime}$, then

$$
\check{\xi}_{\omega}\left(X^{\prime} Y\right)=\left(\omega \circ X^{\prime}\right) \check{\xi}_{\omega}(Y) \equiv \lambda\left(\omega \circ X^{\prime}\right) \check{\xi}_{\omega}(Y)=\left(\omega^{-1} \circ \lambda\right)\left(X^{\prime}\right) \check{\xi}_{\omega}(Y)=0 .
$$

Hence $\chi\left(\check{\xi}_{\omega}\left(X^{\prime} Y\right)\right)=0$, unless $\omega \in \mathfrak{R}_{\lambda}=\mathfrak{S}_{\lambda}$. Let us now prove another lemma.

Lemma 1.8. For any $\sigma \in \mathfrak{S}_{\lambda}$, we have $\check{\xi}_{\sigma}(Y) \equiv Y$.

Proof. The subgroup $\mathfrak{S}_{\lambda} \subset \mathfrak{S}$ is generated by the reflections $\sigma_{\alpha}$ corresponding to the positive roots $\alpha \in \Delta^{+}$such that $(\lambda+\rho)\left(H_{\alpha}\right)=0$; see [B. Proposition V.3.2]. Fix any such $\alpha$ and write $\alpha=\sigma\left(\alpha_{c}\right)$, where $\alpha_{c}$ is a simple root and $\sigma \in \mathfrak{S}$. Since $Y \in \overline{\mathrm{Z}}^{0}$, then

$$
\check{\xi}_{\sigma_{\alpha}}(Y)=\left(\check{\xi}_{\sigma} \check{\xi}_{c} \check{\xi}_{\sigma^{-1}}\right)(Y) \text {. }
$$

Consider the element $Y^{\prime}=\check{\xi}_{\sigma^{-1}}(Y)$ of $\overline{\mathrm{Z}}$. By our assumptions on $Y$, the element $Y^{\prime}$ is the image of some element $A^{\prime} \in \mathrm{A}^{0}$ under the projection $\overline{\mathrm{A}} \rightarrow \overline{\mathrm{Z}}$. Consider the $\mathfrak{s l}_{2}$-subalgebra $\mathfrak{g}_{c} \subset \mathfrak{g}$. First suppose that $A^{\prime}$ belongs to an irreducible $\mathfrak{g}_{c^{-}}$ submodule of $\mathrm{A}$. If $A^{\prime} \neq 0$, then this submodule has an odd dimension, say $2 j+1$. 
By the calculation made at the end of Subsection 1.4, the operator $\check{\xi}_{c}$ maps the coset of $A^{\prime}$ in $\overline{\mathrm{Z}}$ to

$$
(-1)^{j} \prod_{i=1}^{j} \frac{H_{c}+i+1}{H_{c}-i+1} \cdot Y^{\prime}
$$

Here we also used the following observation: because $A^{\prime} \in \mathrm{A}^{0}$ belongs to an irreducible $\mathfrak{g}_{c}$-submodule of dimension $2 j+1$, we have $\widehat{\sigma}_{c}\left(A^{\prime}\right)=(-1)^{j} A^{\prime}$. Thus

$$
\check{\xi}_{\sigma_{\alpha}}(Y)=(-1)^{j} \prod_{i=1}^{j} \frac{\sigma \circ H_{c}+i+1}{\sigma \circ H_{c}-i+1} \cdot \check{\xi}_{\sigma}\left(Y^{\prime}\right)=(-1)^{j} \prod_{i=1}^{j} \frac{\sigma \circ H_{c}+i+1}{\sigma \circ H_{c}-i+1} \cdot Y .
$$

But

$$
\begin{aligned}
\lambda\left(\sigma \circ H_{c}\right) & =\left(\sigma^{-1} \circ \lambda\right)\left(H_{c}\right)=\left(\sigma^{-1}(\lambda+\rho)-\rho\right)\left(H_{c}\right) \\
& =(\lambda+\rho)\left(\sigma\left(H_{c}\right)\right)-\rho\left(\sigma\left(H_{c}\right)\right)=(\lambda+\rho)\left(H_{\alpha}\right)-1=-1,
\end{aligned}
$$

so that

$$
\check{\xi}_{\sigma_{\alpha}}(Y) \equiv(-1)^{j} \prod_{i=1}^{j} \frac{-1+i+1}{-1-i+1} \cdot Y=Y .
$$

The assumption that $A^{\prime}$ belongs to an irreducible $\mathfrak{g}_{c}$-submodule can now be removed without any loss of generality. Lemma 1.8 is thus proved for any $\sigma=\sigma_{\alpha}$.

Using Lemma 1.8, for any $\sigma \in \mathfrak{S}_{\lambda}$ we now get

$$
\check{\xi}_{\sigma}\left(X^{\prime} Y\right)=\left(\sigma \circ X^{\prime}\right) \check{\xi}_{\sigma}(Y) \equiv \lambda\left(\sigma \circ X^{\prime}\right) Y=\left(\sigma^{-1} \circ \lambda\right)\left(X^{\prime}\right) Y=\lambda\left(X^{\prime}\right) Y \equiv X^{\prime} Y .
$$

This argument completes the proof of the equality $\chi(Y)=\chi(Z)$ and hence that of Proposition 1.6.

1.6. Irreducible S-modules. In this subsection we introduce a class of irreducible S-modules, to which we can apply Proposition 1.6. Let $K$ be any left A-module. Since $\mathrm{U}(\mathfrak{g})$ is a subalgebra of $\mathrm{A}$, we can regard $K$ as a $\mathfrak{g}$-module by restriction. Suppose the action of the subalgebra $\mathfrak{t} \subset \mathfrak{g}$ on $K$ is semisimple, so that $K$ splits into a direct sum of the weight subspaces $K^{\lambda}$, where $\lambda$ ranges over $\mathfrak{t}^{*}$. Also suppose the action of the subalgebra $\mathfrak{n}^{\prime} \subset \mathfrak{g}$ on $K$ is locally nilpotent, so that for any $v \in K$ there is a positive integer $s$ such that $\left(\mathfrak{n}^{\prime}\right)^{s} v=\{0\}$.

Consider the space $K_{\mathfrak{n}}$ of $\mathfrak{n}$-coinvariants of $K$. Take any $\lambda \in \mathfrak{t}^{*}$ and consider the weight subspace $K_{\mathfrak{n}}^{\lambda} \subset K_{\mathfrak{n}}$. Denote by $N$ the subspace of $K$ consisting of all $\mathfrak{n}^{\prime}$-invariants of weight $\lambda$. By restricting the canonical projection $K \rightarrow K_{\mathfrak{n}}$ to the subspace $N \subset K$, we get a natural linear map $N \rightarrow K_{\mathfrak{n}}^{\lambda}$. In general, the restriction map may not be bijective.

Now suppose that the weight $\lambda+\rho$ is dominant. It turns out that then the map $N \rightarrow K_{\mathfrak{n}}^{\lambda}$ is bijective. To define the inverse map, take any coset $f \in K_{\mathfrak{n}}^{\lambda}$ and choose its representative $u \in K$. We may assume that $u \in K^{\lambda}$. Then we can define a linear map $K_{\mathfrak{n}}^{\lambda} \rightarrow N$ by mapping $f \mapsto P u$. Here we use the properties (1.6), the second property in (1.8), the local nilpotency of $K$ relative to $\mathfrak{n}^{\prime}$, and the dominance of $\lambda+\rho$. This map is the right and left inverse to the projection $N \rightarrow K_{\mathfrak{n}}^{\lambda}$, due to the first and the second properties in (1.7), respectively. See also the proofs of the properties (1.6) we referred to. 
Since $\lambda+\rho$ is dominant, the weight subspace $K_{\mathfrak{n}}^{\lambda} \subset K_{\mathfrak{n}}$ has a structure of a module over the algebra $\mathrm{S}$. The action of $\mathrm{S}$ on $K_{\mathfrak{n}}^{\lambda}$ will be denoted by $\diamond$ as in Subsection 1.2 , and is defined by

$$
X \diamond f=X P f \quad \text { for } \quad X \in \mathrm{S} \quad \text { and } \quad f \in K_{\mathfrak{n}}^{\lambda} .
$$

On the right-hand side of this equality, we use the action of the algebra $\mathrm{A}$ on $K$.

Proposition 1.9. Suppose that $K$ is an irreducible A-module, while the weight $\lambda+\rho$ is dominant. Then $K_{\mathfrak{n}}^{\lambda}$ is an irreducible $\mathrm{S}$-module.

Proof. Take any two cosets $f, g \in K_{\mathfrak{n}}^{\lambda}$ and choose their representatives $u, v \in K$. We may assume that both $u, v \in K^{\lambda}$. Determine the element $P u \in K^{\lambda}$ as above. Because $K$ is an irreducible A-module, we can find an element $A \in \mathrm{A}^{0}$ such that $A P u=v$. Let $X \in \mathrm{Z}$ be the coset of $A$. Then we have $X \in \mathrm{S}$ and $X \diamond f=g$.

\section{ShapovalOV FORMS}

2.1. Howe systems. Now suppose that the reductive Lie algebra $\mathfrak{g}$ is symmetric. That is, $\mathfrak{g}$ is equipped with an involutive automorphism identical on the Cartan subalgebra $\mathfrak{t}$ and preserving each of the nilpotent subalgebras $\mathfrak{n}$ and $\mathfrak{n}^{\prime}$. We have the Cartan decomposition $\mathfrak{g}=\mathfrak{g}_{+} \oplus \mathfrak{g}_{-}$, where $\mathfrak{g}_{+}$and $\mathfrak{g}_{-}$are the eigenspaces of this automorphism with eigenvalues 1 and -1 . Then $\mathfrak{g}_{+}$is a Lie subalgebra of $\mathfrak{g}$ containing $\mathfrak{t}$, and

$$
\left[\mathfrak{g}_{+}, \mathfrak{g}_{-}\right] \subset \mathfrak{g}_{-} \text {and }\left[\mathfrak{g}_{-}, \mathfrak{g}_{-}\right] \subset \mathfrak{g}_{+} .
$$

Note that then the involutive anti-automorphism $\varepsilon$ of $\mathfrak{g}$ preserves the decomposition $\mathfrak{g}=\mathfrak{g}_{+} \oplus \mathfrak{g}_{-}$. This decomposition gives decompositions of the nilpotent subalgebras: we have $\mathfrak{n}=\mathfrak{n}_{+} \oplus \mathfrak{n}_{-}$and $\mathfrak{n}^{\prime}=\mathfrak{n}_{+}^{\prime} \oplus \mathfrak{n}_{-}^{\prime}$, where

$$
\mathfrak{n}_{+}=\mathfrak{n} \cap \mathfrak{g}_{+}, \quad \mathfrak{n}_{-}=\mathfrak{n} \cap \mathfrak{g}_{-}, \quad \mathfrak{n}_{+}^{\prime}=\mathfrak{n}^{\prime} \cap \mathfrak{g}_{+}, \quad \mathfrak{n}_{-}^{\prime}=\mathfrak{n}^{\prime} \cap \mathfrak{g}_{-} .
$$

Let $U$ be a finite-dimensional complex vector space and $\theta \in\{1,-1\}$. Depending on whether $\theta=1$ or $\theta=-1$, denote by $\mathrm{H}(U)$ the Weyl algebra or the Clifford algebra of $U \oplus U^{*}$. This is the complex associative unital algebra generated by the elements of $U$ and of the dual space $U^{*}$ subject to the relations for $u, v \in \mathrm{U}$ and $u^{\prime}, v^{\prime} \in U^{*}$

$$
v u=\theta u v, \quad v^{\prime} u^{\prime}=\theta u^{\prime} v^{\prime}, \quad u^{\prime} u-\theta u u^{\prime}=u^{\prime}(u) .
$$

Equip the vector space $W=U \oplus U^{*}$ with the bilinear form $B$, symmetric if $\theta=-1$ and alternating if $\theta=1$, such that $B(u, v)=B\left(u^{\prime}, v^{\prime}\right)=0$ and $B\left(u, u^{\prime}\right)=$ $u^{\prime}(u)$. The relations (2.1) are then equivalent to the relations for all $w, w^{\prime} \in W$,

$$
w^{\prime} w-\theta w w^{\prime}=B\left(w, w^{\prime}\right) .
$$

Then any isotropic subspace $V \subset W$ generates a subalgebra of $\mathrm{H}(U)$, which is a free commutative algebra if $\theta=1$, or free skew-commutative if $\theta=-1$. This subalgebra will be denoted by $\mathrm{P}(V)$. In particular, the algebra $\mathrm{H}(U)$ contains two distinguished subalgebras $\mathrm{P}(U)$ and $\mathrm{P}\left(U^{*}\right)$, generated by the elements of $U$ and of $U^{*}$, respectively.

Further, equip the vector space $W$ with a grading so that the direct summands $U$ and $U^{*}$ of $W$ have degrees 1 and -1 , respectively. This grading naturally extends to a $\mathbb{Z}$-grading on the algebra $\mathrm{H}(U)$. In particular, the subalgebras $\mathrm{P}(U)$ and $\mathrm{P}\left(U^{*}\right)$ of $\mathrm{H}(U)$ are graded, respectively, by nonnegative and nonpositive integers. 
Now suppose there is a homomorphism of associative algebras $\zeta: \mathrm{U}(\mathfrak{g}) \rightarrow \mathrm{H}(U)$. Further suppose there is an action of the group $\mathrm{G}$ on the vector space $W$ preserving the bilinear form $B$. Since the form is preserved, this action extends uniquely to an action of $\mathrm{G}$ by automorphisms of the algebra $\mathrm{H}(U)$. In particular, we get an action of the Lie algebra $\mathfrak{g}$ on $\mathrm{H}(U)$, and the weight decomposition

$$
\mathrm{H}(U)=\underset{\lambda \in \mathfrak{t}^{*}}{\oplus} \mathrm{H}(U)^{\lambda} .
$$

Choose a nondegenerate symmetric bilinear form $\langle$,$\rangle on the vector space U$. Using it, define a linear map $\varepsilon: W \rightarrow W$ such that $\varepsilon: U \rightarrow U^{*}, \varepsilon: U^{*} \rightarrow U$ while

$$
\varepsilon(u)(v)=\langle u, v\rangle \quad \text { and } \quad\left\langle u, \varepsilon\left(u^{\prime}\right)\right\rangle=u^{\prime}(u)
$$

for $u, v \in U$ and $u^{\prime} \in U^{*}$. The map $\varepsilon$ is involutive and for all $w, w^{\prime} \in W$ satisfies

$$
B\left(\varepsilon(w), \varepsilon\left(w^{\prime}\right)\right)=-\theta B\left(w, w^{\prime}\right) .
$$

Therefore this map admits a unique extension to an involutive anti-automorphism of the algebra $\mathrm{H}(U)$. We denote the extension by $\varepsilon$ again.

We shall say that the homomorphism $\zeta: \mathrm{U}(\mathfrak{g}) \rightarrow \mathrm{H}(U)$ and the action of $\mathrm{G}$ on $W$ form a Howe system on $U$ if the following six conditions are satisfied:

(1) the $\operatorname{map} \zeta: \mathrm{U}(\mathfrak{g}) \rightarrow \mathrm{H}(U)$ is G-equivariant;

(2) the action of $\mathfrak{g}$ on $\mathrm{H}(U)$ corresponding to that of $\mathrm{G}$ is adjoint to $\zeta$;

(3) $\varepsilon(\zeta(X))=\zeta(\varepsilon(X))$ for all $X \in \mathfrak{g}$;

(4) $\zeta(\mathfrak{n}) \subset U \cdot \mathrm{H}(U)$ and $\zeta(\mathfrak{t}) \subset \mathbb{C} \cdot 1+U \cdot \mathrm{H}(U)$;

(5) $\left[\zeta\left(\mathfrak{g}_{+}\right), U\right] \subset U$ and $\left[\zeta\left(\mathfrak{n}_{-}\right), U^{*}\right] \subset U$ while $\left[\zeta\left(\mathfrak{n}_{-}\right), U\right]=\{0\}$;

(6) any weight element of $\mathrm{P}(U)$ has a $\mathbb{Z}$-degree uniquely defined by the weight.

The property (2) of a Howe system means that the action of Lie algebra $\mathfrak{g}$ on $\mathrm{H}(U)$ corresponding to that of $\mathrm{G}$ is given by

$$
X: Y \mapsto[\zeta(X), Y] \text { for } X \in \mathfrak{g} \text { and } Y \in \mathrm{H}(U) .
$$

The properties (2) and (3) imply that the automorphism $\varepsilon$ of $\mathrm{H}(U)$ changes the signs of the degree and of the weight. Here and in (6) we refer to the $\mathbb{Z}$-grading on $\mathrm{H}(U)$ and the weight decomposition (2.3). By combining (3) and (4), (5) we get two more properties:

(7) $\zeta\left(\mathfrak{n}^{\prime}\right) \subset \mathrm{H}(U) \cdot U^{*}$ and $\zeta(\mathfrak{t}) \subset \mathbb{C} \cdot 1+\mathrm{H}(U) \cdot U^{*}$;

(8) $\left[\zeta\left(\mathfrak{g}_{+}\right), U^{*}\right] \subset U^{*}$ and $\left[\zeta\left(\mathfrak{n}_{-}^{\prime}\right), U\right] \subset U^{*}$ while $\left[\zeta\left(\mathfrak{n}_{-}^{\prime}\right), U^{*}\right]=\{0\}$.

The properties (5) and (8) imply that for $X \in \mathfrak{g}_{+}, \mathfrak{n}_{-}, \mathfrak{n}_{-}^{\prime}$ the adjoint operators $\operatorname{ad}_{\zeta(X)}$ on $\mathrm{H}(U)$ have the degrees $0,2,-2$, respectively.

Later on we will work with known examples [H1] of Howe systems. The list of (1) to (8) summarizes the common properties of these examples that we shall use. Now fix any Howe system on $U$ and set

$$
\mathrm{A}=\mathrm{U}(\mathfrak{g}) \otimes \mathrm{H}(U) .
$$

The group $\mathrm{G}$ acts (diagonally) by automorphisms of the algebra A. Let us identify $\mathrm{U}(\mathfrak{g})$ with subalgebra of A generated by the elements

$$
X \otimes 1+1 \otimes \zeta(X) \quad \text { where } \quad X \in \mathfrak{g} .
$$

Then set

$$
\mathrm{V}=1 \otimes \mathrm{H}(U) .
$$

The condition (2) on a Howe system implies that the condition (c) on the algebra A is satisfied. Note that in this case $\mathrm{V}$ is a subalgebra of A, not only a vector subspace. 
In this section we will investigate the Mickelsson algebras $\mathrm{R}$ and $\overline{\mathrm{Z}}$ corresponding to the associative algebra (2.5) determined by any Howe system. We will usually identify any element $X \in \mathfrak{g}$ with the element (2.6) of A, and any element $Y \in \mathrm{H}(U)$ with $1 \otimes Y \in \mathrm{A}$. Note that for $X \in \mathfrak{g}$, the commutator $[X, Y]$ in the algebra A equals $[\zeta(X), Y] \in \mathrm{H}(U)$.

2.2. Shapovalov form on double coset algebra. Let us extend the map $\varepsilon$ from $\mathrm{H}(U)$ to the algebra A defined by (2.5), so that under the extension $X \otimes Y \mapsto$ $\varepsilon(X) \otimes \varepsilon(Y)$ for $X \in \mathrm{U}(\mathfrak{g})$ and $Y \in \mathrm{H}(U)$. Here the symbol $\varepsilon$ in the first tensor factor denotes the Chevalley anti-involution on $\mathrm{U}(\mathfrak{g})$. The same symbol $\varepsilon$ in the second tensor factor denotes the involutive anti-automorphism of $\mathrm{H}(U)$ defined by (2.4). The extended map is an involutive anti-automorphism of the algebra A. Its restriction to the subalgebra $U(\mathfrak{g})$ generated by the elements (2.6) coincides with the Chevalley anti-involution on $\mathrm{U}(\mathfrak{g})$, due to the property (3) of a Howe system. This anti-automorphism further extends from A to $\bar{A}$.

Since the latter extension preserves the subspace $\overline{\mathrm{J}}+\overline{\mathrm{J}}^{\prime} \subset \overline{\mathrm{A}}$, it defines an involutive linear map $\varepsilon: \overline{\mathrm{Z}} \rightarrow \overline{\mathrm{Z}}$. Because $\varepsilon(P)=P$ where $P$ is the extremal projector for $\mathfrak{g}$, this map is an anti-automorphism of the double coset algebra $\bar{Z}$. We will denote it by the same symbol $\varepsilon$. Clearly, $\varepsilon$ maps the subalgebra $\mathrm{S} \subset \overline{\mathrm{Z}}$ to itself. Denote by K the right ideal of the algebra $\bar{Z}$, generated by the cosets of all elements $u \in U$. Similarly, denote by $\mathrm{K}^{\prime}$ the left ideal of the algebra $\overline{\mathrm{Z}}$, generated by the cosets of all elements $u^{\prime} \in U^{*}$. We identify the elements $u, u^{\prime} \in \mathrm{H}(U)$ with the elements $1 \otimes u, 1 \otimes u^{\prime} \in \mathrm{A}$, respectively.

Lemma 2.1. (i) The subspace $\mathrm{K} \subset \overline{\mathrm{Z}}$ is spanned by the cosets of products $u X$ in $\overline{\mathrm{A}}$ where $u \in U$ and $X \in \overline{\mathrm{A}}$.

(ii) $\mathrm{K}^{\prime}$ is spanned by the cosets of products $X u^{\prime}$ in $\overline{\mathrm{A}}$ where $u^{\prime} \in U^{*}, X \in \overline{\mathrm{A}}$.

Proof. Take any $u \in U$ and $X \in \overline{\mathrm{A}}$. The property (5) of a Howe system implies that for any $A \in \mathfrak{n}$ the commutator $[u, A] \in U$. We may assume that $u$ is a weight element, say $u \in U^{\lambda}$ for some $\lambda \in \mathfrak{t}^{*}$. If $A \in \mathfrak{n}$ is also a weight element, then $[u, A]$ has a weight less than $\lambda$. The inequality $\mu<\lambda$ for some $\mu \in \mathfrak{t}^{*}$ means that the difference $\lambda-\mu$ is a nonzero sum of simple roots in $\Delta^{+}$with nonnegative integral coefficients. By the first relation in (1.7),

$$
u P X \in u X+\sum_{v} v Y+\overline{\mathrm{J}}
$$

where $v$ ranges over a certain finite subset of $U$ and has a weight $\mu<\lambda$, while $Y \in \overline{\mathrm{A}}$ corresponds to $v$. But (2.7) shows that the product of the cosets of $u$ and of $X$ in the algebra $\overline{\mathrm{Z}}$ is the coset of

$$
u X+\sum_{v} v Y
$$

Further, we can recursively invert (2.7) and get the relation in the algebra $\overline{\mathrm{A}}$,

$$
u X \in u P X+\sum_{w} w P Z+\overline{\mathrm{J}}
$$

where $w$ ranges over some finite subset of $U$ and has a weight less than $\lambda$, while $Z \in \overline{\mathrm{A}}$ corresponds to $w$. So the coset in $\overline{\mathrm{Z}}$ of the product $u X$ in the algebra $\overline{\mathrm{A}}$ is the coset of

$$
u P X+\sum_{w} w P Z
$$


and therefore belongs to the ideal $\mathrm{K}$ of the algebra $\overline{\mathrm{Z}}$. This completes the proof of part (i) of the lemma. The proof of part (ii) is very similar and is omitted here.

Corollary 2.2. For any $Z \in \overline{\mathrm{Z}}$ there is a unique element $(Z)_{0} \in \overline{\mathrm{U}(\mathfrak{t})}$ such that

$$
Z-(Z)_{0} \in \mathrm{K}+\mathrm{K}^{\prime} .
$$

Proof. Choose any ordered basis in each of the vector spaces $\mathfrak{n}, \mathfrak{n}^{\prime}, U, U^{\prime}$, and $\mathfrak{t}$. By the classical Poincaré-Birkhoff-Witt theorem, each of the vector spaces $U(\mathfrak{n}), U\left(\mathfrak{n}^{\prime}\right)$, $\mathrm{P}(U), \mathrm{P}\left(U^{*}\right)$, and $\mathrm{U}(\mathfrak{t})$ has a basis made of all monomials in the corresponding basis vectors. If $A, A^{\prime}, B, B^{\prime}$, and $C$ run through these monomials, then the products $A B C B^{\prime} A^{\prime}$ form a basis in $\mathrm{A}$. So the vector space $\overline{\mathrm{Z}}$ is spanned by the cosets of products in $\overline{\mathrm{A}}$ of the form $B D B^{\prime}$, where $B$ and $B^{\prime}$ are as above whereas $D \in \overline{\mathrm{U}(\mathfrak{t})}$. By Lemma 2.1, any such coset belongs to $\mathrm{K}+\mathrm{K}^{\prime}$ unless $B=B^{\prime}=1$. Thus, $(Z)_{0} \in \overline{\mathrm{U}(\mathfrak{t})}$ exists for any $Z \in \overline{\mathrm{Z}}$.

Now suppose that $(Z)_{0} \in \overline{\mathrm{U}(\mathfrak{t})}$ is not unique. Then Lemma 2.1 implies the existence of a nonzero element of $\overline{\mathrm{U}(\mathfrak{t})}$ which belongs to the sum of the right ideal of $\overline{\mathrm{A}}$ generated by $\mathfrak{n}$ and $U$, and of the left ideal of $\overline{\mathrm{A}}$ generated by $\mathfrak{n}^{\prime}$ and $U^{*}$. But this is not possible due to existence of the basis in A described above.

Regard $\overline{\mathrm{Z}}$ as a left $\overline{\mathrm{U}(\mathfrak{t})}$-module. Define the Shapovalov form $S: \overline{\mathrm{Z}} \times \overline{\mathrm{Z}} \rightarrow \overline{\mathrm{U}(\mathfrak{t})}$ by

$$
S(X, Y)=(\varepsilon(X) \diamond Y)_{0} \quad \text { for } \quad X, Y \in \overline{\mathrm{Z}} .
$$

It is symmetric, contravariant, and $\overline{\mathrm{U}(\mathfrak{t})}$-linear by definition. That is, we have

$$
\begin{aligned}
S(X, Y) & =S(Y, X) ; \\
S(Z \diamond X, Y) & =S(X, \varepsilon(Z) \diamond Y) \quad \text { for } \quad Z \in \overline{\mathrm{Z}} ; \\
S(Z X, Y) & =Z S(X, Y) \quad \text { for } \quad Z \in \overline{\mathrm{U}(\mathfrak{t})} .
\end{aligned}
$$

Note that

$$
S(X, Y)=0 \quad \text { when } \quad Y \in \mathrm{K}^{\prime} .
$$

2.3. Shapovalov form on coinvariant space. Let $\mu \in \mathfrak{t}^{*}$ be any weight. Denote by $\mathrm{J}_{\mu}^{\prime}$ the left ideal of $\mathrm{A}$, generated by the elements $X$ of $\mathfrak{n}^{\prime}$, and by the elements $H-\mu(H)$, where $H \in \mathfrak{t}$. Here we identify any element $X$ of $\mathfrak{g}$ with the element (2.6) of the algebra A. Further, denote by $\mathrm{J}_{\mu}^{\prime \prime}$ the left ideal of A, generated by the elements $X \otimes 1$, where $X \in \mathfrak{n}^{\prime}$, and the elements $H \otimes 1-\mu(H)$, where $H \in \mathfrak{t}$. Let I be the left ideal of A generated by the elements of $U^{*} \subset \mathrm{H}(U)$. The first inclusion in the property (7) of a Howe system implies that $X-X \otimes 1 \in \mathrm{I}$ for $X \in \mathfrak{n}^{\prime}$. The second inclusion in the property (7) implies that there is a weight $\kappa \in \mathfrak{t}^{*}$ such that

$$
\zeta(H)-\kappa(H) \in \mathrm{H}(U) \cdot U^{*} \text { for all } H \in \mathfrak{t} .
$$

Hence $H-H \otimes 1-\kappa(H) \in \mathrm{I}$ for $H \in \mathfrak{t}$. Therefore

$$
\mathrm{J}_{\mu}^{\prime \prime}+\mathrm{I}=\mathrm{J}_{\mu+\kappa}^{\prime}+\mathrm{I} .
$$

Let $\mathrm{A}_{\mu}$ be the quotient space of A relative to the left ideal $\mathrm{J}_{\mu}^{\prime \prime}+\mathrm{I}$. Let $\mathrm{M}_{\mu}$ be the vector space of double cosets of A relative to the left ideal $\mathrm{J}_{\mu}^{\prime \prime}+\mathrm{I}$ and the right ideal $\mathrm{J}$ :

$$
\mathrm{A}_{\mu}=\mathrm{A} /\left(\mathrm{J}_{\mu}^{\prime \prime}+\mathrm{I}\right), \quad \mathrm{M}_{\mu}=\mathrm{A}_{\mu} / \mathrm{J}=\mathrm{A} /\left(\mathrm{J}_{\mu}^{\prime \prime}+\mathrm{I}+\mathrm{J}\right) .
$$


Regard the ring $\mathrm{P}(U)$ as a left $\mathrm{H}(U)$-module, by identifying this ring with the quotient of $\mathrm{H}(U)$ over the left ideal generated by $U^{*}$. Thus for $X \in \mathrm{H}(U)$ and $u, v \in \mathrm{P}(U)$

$$
X(u)=v \quad \text { if } \quad X u-v \in \mathrm{H}(U) \cdot U^{*} .
$$

Then we can identify the A-module $\mathrm{A}_{\mu}$ with the tensor product $M_{\mu} \otimes \mathrm{P}(U)$ where $M_{\mu}$ is the Verma module of the algebra $\mathrm{U}(\mathfrak{g})$ appearing as the first tensor factor in (2.5). The vector space $\mathrm{M}_{\mu}$ gets identified with the space of coinvariants

$$
\left(M_{\mu} \otimes \mathrm{P}(U)\right)_{\mathfrak{n}} \text {. }
$$

The Cartan subalgebra $\mathfrak{t} \subset \mathfrak{g}$ acts on this space via left multiplication in A. This space is a left module over the Mickelsson algebra $\mathrm{R}$. It is generated by the image of

$$
1_{\mu} \otimes 1 \in M_{\mu} \otimes \mathrm{P}(U),
$$

where $1_{\mu}$ is the highest weight vector of the Verma module $M_{\mu}$. By using the equality (2.13), any element $H \in \mathfrak{t}$ acts on the vector (2.17) as multiplication by $(\mu+\kappa)(H)$. Hence the image of the vector (2.17) in $\mathrm{M}_{\mu}$ belongs to the weight subspace $\mathrm{M}_{\mu}^{\mu+\kappa}$. Due to the condition (6) on a Howe system, the subspace $\mathrm{M}_{\mu}^{\mu+\kappa} \subset$ $\mathrm{M}_{\mu}$ is one dimensional and is spanned by the image of (2.17). Note that there is an isomorphism of vector spaces $\mathrm{P}(U) \rightarrow \mathrm{M}_{\mu}$. It can be defined by assigning to any $u \in \mathrm{P}(U)$ the coset of $1 \otimes u \in \mathrm{A}$ in the quotient $\mathrm{M}_{\mu}=\mathrm{A} /\left(\mathrm{J}_{\mu}^{\prime}+\mathrm{I}+\mathrm{J}\right)$. This isomorphism will be denoted by $\iota_{\mu}$.

Now consider the left ideals of the algebra $\bar{A}$,

$$
\overline{\mathrm{J}}_{\mu}^{\prime \prime}=\overline{\mathrm{U}(\mathfrak{t})} \mathrm{J}_{\mu}^{\prime \prime} \quad \text { and } \quad \overline{\mathrm{I}}=\overline{\mathrm{U}(\mathfrak{t})} \mathrm{I} .
$$

Suppose the weight $\mu+\kappa \in \mathfrak{t}^{*}$ is generic, that is, $(\mu+\kappa)\left(H_{\alpha}\right) \notin \mathbb{Z}$ for all $\alpha \in \Delta^{+}$. Then the spaces of double cosets $\overline{\mathrm{A}} /\left(\overline{\mathrm{J}}_{\mu}^{\prime \prime}+\overline{\mathrm{I}}+\overline{\mathrm{J}}\right)$ and $\mathrm{A} /\left(\mathrm{J}_{\mu}^{\prime \prime}+\mathrm{I}+\mathrm{J}\right)=\mathrm{M}_{\mu}$ are naturally isomorphic. Here we once again use the equality (2.13). Further, $\mathrm{M}_{\mu}$ is isomorphic to the left $\bar{Z}$-module, defined as the quotient of the algebra $\bar{Z}$ by the left ideal generated by the cosets of the elements of $1 \otimes U^{*}$ and the cosets of the elements $H-(\mu+\kappa)(H)$, where $H \in \mathfrak{t}$. Here we use the isomorphism of the algebras $\overline{\mathrm{R}}$ and $\overline{\mathrm{Z}}$; see Proposition 1.1 and remarks made immediately after stating it. Accordingly, we will use the symbol $\diamond$ to denote the action of the algebra $\overline{\mathrm{Z}}$ on $\mathrm{M}_{\mu}$. Then any element of $\mathrm{M}_{\mu}$ can be presented as $Z \diamond \iota_{\mu}(1)$ for some $Z \in \overline{\mathrm{Z}}$. Since $\iota_{\mu}(1) \in \mathrm{M}_{\mu}^{\mu+\kappa}$, we get $Z \diamond \iota_{\mu}(1) \in \mathrm{M}_{\mu}^{\lambda}$ if $Z \in \overline{\mathrm{Z}}^{\nu}$ and

$$
\nu=\lambda-\mu-\kappa .
$$

We may also choose $Z$ from the image in $\overline{\mathrm{Z}}$ of the subalgebra $1 \otimes \mathrm{P}(U) \subset \overline{\mathrm{A}}$. Indeed, due to (1.7) the cosets of $(1 \otimes u) P$ and $1 \otimes u$ in $\mathrm{M}_{\mu}$ coincide for any element $u \in \mathrm{P}(U)$.

Due to (2.8)-(2.11), for a generic weight $\mu+\kappa$ the Shapovalov form $S: \overline{\mathrm{Z}} \otimes \overline{\mathrm{Z}} \rightarrow$ $\overline{\mathrm{U}(\mathfrak{t})}$ defines a symmetric contravariant form $S_{\mu}: \mathrm{M}_{\mu} \otimes \mathrm{M}_{\mu} \rightarrow \mathbb{C}$ by setting

$$
S_{\mu}\left(X \diamond \iota_{\mu}(1), Y \diamond \iota_{\mu}(1)\right)=S(X, Y)(\mu+\kappa) \quad \text { for } \quad X, Y \in \overline{\mathrm{Z}} .
$$

Here $S(X, Y)(\mu+\kappa)$ is the evaluation at $\mu+\kappa$ of an element of $\overline{\mathrm{U}(\mathfrak{t})}$, regarded as a rational function on $\mathfrak{t}^{*}$. The contravariance of the form $S_{\mu}$ means the equality

$$
S_{\mu}(Z \diamond f, g)=S_{\mu}(f, \varepsilon(Z) \diamond g) \quad \text { for } \quad f, g \in \mathrm{M}_{\mu} \quad \text { and } \quad Z \in \overline{\mathrm{Z}} \text {. }
$$

Note that if $f=\iota_{\mu}(u)$ and $g=\iota_{\mu}(v)$ for some $u$ and $v$ from $\mathrm{P}(U)$, then

$$
S_{\mu}(f, g)=S(X, Y)(\mu+\kappa)
$$


where $X$ and $Y$ are, respectively, the images of $1 \otimes u$ and $1 \otimes v$ under the projection map $\mathrm{A} \rightarrow \mathrm{Z}$. This follows from the remark made at the end of the last paragraph.

In Subsection 2.1 we chose a nondegenerate symmetric bilinear form $\langle$,$\rangle on U$. Let us now extend this form from $U$ to $\mathrm{P}(U)$ in a natural way, as follows. Choose an orthonormal basis $u_{1}, \ldots, u_{n}$ of $U$. Thus $\left\langle u_{i}, u_{j}\right\rangle=\delta_{i j}$ for $i, j=1, \ldots, n$. Put

$$
\left\langle u_{1}^{p_{1}} \cdots u_{n}^{p_{n}}, u_{1}^{q_{1}} \cdots u_{n}^{q_{n}}\right\rangle=\prod_{k=1}^{n} \delta_{p_{k} q_{k}} p_{k} !,
$$

where for every $k=1, \ldots, n$ we take $p_{k}, q_{k} \in\{0,1,2, \ldots\}$ in the case $\theta=1$, whereas $p_{k}, q_{k} \in\{0,1\}$ in the case $\theta=-1$. This form on $\mathrm{P}(U)$ is uniquely determined by setting $\langle 1,1\rangle=1$ and by declaring that the transpose to the operator of left multiplication by any $u_{k}$ is given by the action of $u_{k}^{\prime} \in U^{*}$. Here $u_{1}^{\prime}, \ldots, u_{n}^{\prime}$ is the basis in $U^{*}$ dual to the basis $u_{1}, \ldots, u_{n}$ in $U$. Thus for each $k=1, \ldots, d$ we have

$$
\left\langle u_{k} u, v\right\rangle=\left\langle u, u_{k}^{\prime} v\right\rangle \text { for } u, v \in \mathrm{P}(U) .
$$

Here we regard $\mathrm{P}(U)$ as a left $\mathrm{H}(U)$-module; see (2.15). Since $u_{k}^{\prime}=\varepsilon\left(u_{k}\right)$, the above-displayed equality implies a more general equality,

$$
\langle X u, v\rangle=\langle u, \varepsilon(X) v\rangle \quad \text { for } \quad u, v \in \mathrm{P}(U) \text { and } X \in \mathrm{H}(U) .
$$

Proposition 2.3. Let $\mu+\kappa \in \mathfrak{t}^{*}$ be generic. Then for any $u, v \in \mathrm{P}(U)$,

$$
S_{\mu}(f, g)=\langle u, \zeta(P[\mu+\rho]) v\rangle \quad \text { if } \quad f=\iota_{\mu}(u) \quad \text { and } \quad g=\iota_{\mu}(v) .
$$

Proof. By definition, the extremal projector $P$ is a product in $\widetilde{U}(\mathfrak{g})$ over the set $\Delta^{+}$ of positive roots equipped with any normal ordering. Any such ordering on $\Delta^{+}$has the following basic property [AST]. Take any $\alpha, \beta \in \Delta^{+}$such that $\left[E_{\alpha}, F_{\beta}\right] \neq 0$. Then $\alpha-\beta$ is a root. The commutator $\left[E_{\alpha}, F_{\beta}\right]$ is proportional to $E_{\alpha-\beta}$ or to $F_{\beta-\alpha}$, respectively, if $\alpha-\beta \in \Delta^{+}$or $\beta-\alpha \in \Delta^{+}$. Now suppose that the root $\alpha$ precedes $\beta$ in the ordering. Then the basic property is that either the positive root $\alpha-\beta$ precedes $\alpha$, or the positive root $\beta-\alpha$ is preceded by $\beta$. Repeatedly using this property and the definition (1.4), we can present the extremal projector $P=P[\rho]$ as the sum in $\widetilde{\mathrm{U}(\mathfrak{g})}$ of the products of the form $A A^{\prime} C$, where $A$ and $A^{\prime}$ are normally ordered monomials in the generators $F_{\alpha}$ and $E_{\alpha}$, respectively. Here $\alpha$ ranges over the set $\Delta^{+}$. These monomials form bases in the vector spaces $\mathrm{U}\left(\mathfrak{n}^{\prime}\right)$ and $\mathrm{U}(\mathfrak{n})$, respectively. Further, any monomial $A A^{\prime}$ appears in the sum $P$ with a unique factor $C \in \overline{\mathrm{U}(\mathfrak{t})}$ on the right of it. This $C$ is a ratio of a complex number to a finite product of factors

$$
H_{\alpha}+\rho\left(H_{\alpha}\right)+s,
$$

where $\alpha$ is a positive root and $s$ is a positive integer. We will also regard the elements of $\overline{\mathrm{U}(\mathfrak{t})}$ as rational functions on $\mathfrak{t}^{*}$. By (2.19) the value $S_{\mu}(f, g)$ of the Shapovalov form equals the sum of the values $(X)_{0}(\mu+\kappa)$, where $X$ is the coset in $\overline{\mathrm{Z}}$ of the product in $\overline{\mathrm{A}}$,

$$
1 \otimes \varepsilon(u) \cdot A A^{\prime} C \cdot 1 \otimes v .
$$

Without loss of generality we may assume that the element $v \in \mathrm{P}(U)$ has a weight, say $\lambda \in \mathfrak{t}^{*}$, relative to the adjoint action of $\mathfrak{t}$ :

$$
[\zeta(H), v]=\lambda(H) v \quad \text { for all } \quad H \in \mathfrak{t} .
$$


Then

$$
C \cdot 1 \otimes v=1 \otimes v \cdot D,
$$

where $D \in \overline{\mathrm{U}(\mathfrak{t})}$ is obtained from $C$ by replacing every factor (2.23), respectively, by

$$
H_{\alpha}+(\lambda+\rho)\left(H_{\alpha}\right)+s \text {. }
$$

Consider the product

$$
1 \otimes \varepsilon(u) \cdot A A^{\prime} \cdot 1 \otimes v
$$

in the algebra $\overline{\mathrm{A}}$. Here $A=F_{\alpha} \cdots F_{\beta}$ and $A=E_{\alpha^{\prime}} \cdots E_{\beta^{\prime}}$ for some positive roots $\alpha, \ldots, \beta$ and $\alpha^{\prime}, \ldots, \beta^{\prime}$. By the definition of the ideals $\overline{\mathrm{J}}$ and $\overline{\mathrm{J}}^{\prime}$ of the algebra $\overline{\mathrm{A}}$, the coset in $\overline{\mathrm{Z}}$ of the product (2.27) coincides with that of the product of commutators

$$
\begin{gathered}
{\left[F_{\alpha}, \ldots,\left[F_{\beta}, 1 \otimes \varepsilon(u)\right] \cdots\right] \cdot\left[E_{\alpha^{\prime}}, \ldots,\left[E_{\beta^{\prime}}, 1 \otimes v\right] \cdots\right]} \\
=1 \otimes\left[\zeta\left(F_{\alpha}\right), \ldots,\left[\zeta\left(F_{\beta}\right), \varepsilon(u)\right] \cdots\right] \cdot 1 \otimes\left[\zeta\left(E_{\alpha^{\prime}}\right), \ldots,\left[\zeta\left(E_{\beta^{\prime}}\right), v\right] \cdots\right] .
\end{gathered}
$$

Denote by $Y$ the coset of the last product. Due to the properties (4) and (7) of a Howe system, then we have $(Y)_{0}=(Z)_{0}$ where $Z$ denotes the coset in $\bar{Z}$ of the product in $\bar{A}$,

$$
1 \otimes\left(\varepsilon(u) \cdot \zeta\left(F_{\alpha}\right) \cdots \zeta\left(F_{\beta}\right) \cdot \zeta\left(E_{\alpha^{\prime}}\right) \cdots \zeta\left(E_{\beta^{\prime}}\right) \cdot v\right)=1 \otimes\left(\varepsilon(u) \zeta\left(A A^{\prime}\right) v\right) .
$$

Note that here $(Z)_{0} \in \mathbb{C} \subset \overline{\mathrm{U}(\mathfrak{t})}$. Further, the adjoint action of the subalgebra $\mathfrak{t} \subset \mathfrak{g}$ normalizes the ideals $\overline{\mathrm{J}}$ and $\overline{\mathrm{J}}^{\prime}$ of $\overline{\mathrm{A}}$. It also normalizes the ideals $\mathrm{K}, \mathrm{K}^{\prime}$ of $\overline{\mathrm{Z}}$. Hence the above argument together with (2.26) implies that $(X)_{0}=(Z D)_{0}=$ $(Z)_{0} D$. Therefore

$$
(X)_{0}(\mu+\kappa)=(Z)_{0} \cdot D(\mu+\kappa)=(Z)_{0} \cdot C(\mu+\kappa+\lambda) .
$$

But by the definitions (2.12) and (2.25) of the weights $\kappa$ and $\lambda$, respectively, for every element $H \in \mathfrak{t}$ we have the relations in the algebra $\mathrm{H}(U)$,

$$
v \cdot(\kappa+\lambda)(H) \in v \cdot(\zeta(H)+\lambda(H))+\mathrm{H}(U) \cdot U^{*}=\zeta(H) \cdot v+\mathrm{H}(U) \cdot U^{*} .
$$

They imply the equality in the left $\mathrm{H}(U)$-module $\mathrm{P}(U)$,

$$
\varepsilon(u) \zeta\left(A A^{\prime}\right) v \cdot C(\mu+\kappa+\lambda)=\varepsilon(u) \zeta\left(A A^{\prime} B\right) v,
$$

where $B \in \overline{\mathrm{U}(\mathfrak{t})}$ is obtained from $C$ by replacing every factor (2.23), respectively, by

$$
H_{\alpha}+(\mu+\rho)\left(H_{\alpha}\right)+s .
$$

Since $\langle 1, U \cdot \mathrm{P}(U)\rangle=0$, the right-hand side of (2.28) can now be written as

$$
\left\langle 1, \varepsilon(u) \zeta\left(A A^{\prime} B\right) v\right\rangle=\left\langle u, \zeta\left(A A^{\prime} B\right) v\right\rangle \text {. }
$$

By using the definition of the element $B \in \overline{\mathrm{U}(\mathfrak{t})}$, we now get Proposition 2.3 ,

Consider again the symmetric bilinear form $\langle$,$\rangle on the vector space \mathrm{P}(U)$. Take the action of the Lie algebra $\mathfrak{g}$ on the vector space $\mathrm{H}(U)$ corresponding to that of the group G. By the property (2) of a Howe system, this action of $\mathfrak{g}$ is adjoint to the homomorphism $\zeta: \mathrm{U}(\mathfrak{g}) \rightarrow \mathrm{H}(U)$. By the properties (5) and (8), the action of the Cartan subalgebra $\mathfrak{t} \subset \mathfrak{g}_{+} \subset \mathfrak{g}$ preserves the subspaces $U$ and $U^{*}$ of $\mathrm{H}(U)$. By the definition (2.4) for any $u \in U$ and $u^{\prime} \in U^{*}$

$$
\left\langle u, \varepsilon\left(u^{\prime}\right)\right\rangle=u^{\prime}(u)=B\left(u, u^{\prime}\right)
$$


while the action of the group $\mathrm{G}$ on the vector space $W=U \bigoplus U^{*}$ preserves the bilinear form $B$ by our assumption. Hence for any $H \in \mathfrak{t}$ and for any $u, u^{\prime}$ as above

$$
\left\langle[\zeta(H), u], \varepsilon\left(u^{\prime}\right)\right\rangle+\left\langle u, \varepsilon\left(\left[\zeta(H), u^{\prime}\right]\right)\right\rangle=0 .
$$

Here $\varepsilon$ is an anti-automorphism of the algebra $\mathrm{H}(U)$ obeying the property (3) of a Howe system. Moreover, $\varepsilon(H)=H$ for the Chevalley anti-involution $\varepsilon$ on $\mathfrak{g}$. Thus

$$
\left.\left\langle[\zeta(H), u], \varepsilon\left(u^{\prime}\right)\right\rangle=\left\langle u,\left[\zeta(H), \varepsilon\left(u^{\prime}\right)\right]\right)\right\rangle .
$$

Since any vector of $U$ can be written as $\varepsilon\left(u^{\prime}\right)$ for some $u^{\prime} \in U^{*}$, the last equality implies that the action of the Cartan subalgebra $\mathfrak{t}$ on $\mathrm{P}(U)$ is self-conjugate with respect to the bilinear form $\langle$,$\rangle . Therefore, because the form \langle$,$\rangle on \mathrm{P}(U)$ is nondegenerate, its restriction to any weight subspace of $\mathrm{P}(U)$ is also nondegenerate.

2.4. Shapovalov form on a weight subspace. Now let $\mu \in \mathfrak{t}^{*}$ be an arbitrary weight. Take the subspace $\mathrm{M}_{\mu}^{\lambda}$ of $\mathrm{M}_{\mu}=\mathrm{A} /\left(\mathrm{J}+\mathrm{J}_{\mu}^{\prime \prime}+\mathrm{I}\right)$ consisting of the elements of weight $\lambda$. The Cartan subalgebra $\mathfrak{t} \subset \mathfrak{g}$ acts on $\mathrm{M}_{\mu}$ via the left multiplication in A by the elements of $\mathrm{U}(\mathfrak{g}) \subset \mathrm{A}$. In this subsection, we will assume that $\lambda+\rho$ is dominant. Then for any $f \in \mathrm{M}_{\mu}^{\lambda}$ and $Z \in \mathrm{Z}$ the element $Z \diamond f \in \mathrm{M}_{\mu}$ is well defined. Indeed, the denominators of $P=P[\rho]$ do not vanish on $\mathrm{M}_{\mu}^{\lambda}$ if $\lambda+\rho$ is dominant. In particular, $\mathrm{M}_{\mu}^{\lambda}$ is a module over the subalgebra $\mathrm{S} \subset \overline{\mathrm{Z}}$. Let the weights $\lambda$ and $\mu$ vary under the constraint that their difference $\lambda-\mu$ is fixed. Then for any given elements $X, Y \in \overline{\mathrm{Z}}$, we can regard $S(X, Y)(\mu+\kappa)$ as a rational function of $\mu \in \mathfrak{t}^{*}$. Now recall the definition (2.18) of weight $\nu$.

Proposition 2.4. Suppose that the weight $\lambda+\rho \in \mathfrak{t}^{*}$ is dominant. Then for any given elements $X, Y \in \mathrm{Z}^{\nu}$ the function $S(X, Y)(\mu+\kappa)$ of $\mu$ has only finite values.

Proof. Let $A, B$ be any representatives in A of the cosets $X, Y$, respectively. Then the $\varepsilon(X) \diamond Y$ is the coset of

$$
\varepsilon(A) P B
$$

where $P=P[\rho] \in \widetilde{\mathrm{U}(\mathfrak{g})}$ is the extremal projector for $\mathfrak{g}$. We assume that the elements $A$ and $B$ of A also have weight $\nu$ relative to the adjoint action of $\mathfrak{t}$.

Any poles of $(\varepsilon(X) \diamond Y)_{0}$ as of a rational function on $\mathfrak{t}^{*}$ may arise only from the denominators of the summands of $P[\rho]$. By the definition (1.4), up to nonzero scalar multipliers, these denominators are products of linear factors of the form $H_{\alpha}+\rho\left(H_{\alpha}\right)+s$ with $s=1,2, \ldots$ and $\alpha \in \Delta^{+}$. Let us move these denominators to the right in (2.29) through the factor $B$, and evaluate the resulting denominators at $\mu+\kappa$. Then the linear factors become

$$
(\mu+\kappa+\nu+\rho)\left(H_{\alpha}\right)+s=(\lambda+\rho)\left(H_{\alpha}\right)+s,
$$

which do not depend on $\mu$ and are nonzero numbers when $\lambda+\rho$ is dominant.

When weight $\mu+\kappa$ is generic, the Shapovalov form $S_{\mu}$ on $\mathrm{M}_{\mu}$ can be defined by equation (2.21), where $f=\iota_{\mu}(u)$ and $g=\iota_{\mu}(v)$ for some $u$ and $v$ from $\mathrm{P}(U)$, while $X$ and $Y$ are the images of $1 \otimes u$ and $1 \otimes v$ under the projection $\mathrm{A} \rightarrow \mathrm{Z}$. The same equation (2.21) can now be used to define an S-contravariant form on the subspace $\mathrm{M}_{\mu}^{\lambda} \subset \mathrm{M}_{\mu}$ for dominant $\lambda+\rho$ and any $\mu$. Indeed, if here $f, g \in \mathrm{M}_{\mu}^{\lambda}$, then $X, Y \in \mathrm{Z}^{\nu}$ so that Proposition 2.4 applies. Denote by $S_{\mu}^{\lambda}$ the bilinear form on the subspace $\mathrm{M}_{\mu}^{\lambda} \subset \mathrm{M}_{\mu}$ defined by (2.21),

$$
S_{\mu}^{\lambda}: \mathrm{M}_{\mu}^{\lambda} \times \mathrm{M}_{\mu}^{\lambda} \rightarrow \mathbb{C}
$$


The contravariance (2.9) of the Shapovalov form on $\bar{Z}$ implies that $S_{\mu}^{\lambda}$ is a contravariant form relative to the action of the subalgebra $\mathrm{S} \subset \overline{\mathrm{Z}}$. Indeed, if $\mu+\kappa$ is generic, then by (2.20) for any $f, g \in \mathrm{M}_{\mu}^{\lambda}$ and $Z \in \mathrm{S}$ we have

$$
S_{\mu}^{\lambda}(Z \diamond f, g)=S_{\mu}^{\lambda}(f, \varepsilon(Z) \diamond g) .
$$

When the weight $\lambda-\mu$ is fixed, both sides of this equality are rational functions of $\mu$. Hence these two rational functions are the same. So the equality (2.30) holds for any $\mu$, provided $\lambda+\rho$ is dominant, which is assumed in this subsection.

In the next lemma, we regard $\mathrm{P}(U) \subset \mathrm{H}(U)$ as subalgebras of $\mathrm{A}$ and use the adjoint action of $\mathfrak{t}$ on A; see the condition (5) on a Howe system. We also identify the elements of $\mathrm{H}(U)$ with their images in the double coset algebra $\bar{Z}$. The weights of these elements in $\bar{Z}$ are taken relative to the adjoint action of $t$. By the condition (2) on a Howe system, these weights are the same as relative to the adjoint action of $\mathfrak{t}$ on $\mathrm{H}(U)$. Recall the definition (2.18).

Lemma 2.5. Suppose for some $f \in \mathrm{M}_{\mu}^{\lambda}$ and $Y \in \mathrm{P}\left(U^{*}\right)^{-\nu}$ we have the equality $Y \diamond f=\iota_{\mu}(1)$ in $\mathrm{M}_{\mu}$. Then for any $X \in \mathrm{P}(U)^{\nu}$, we get $(X Y) \diamond f=\iota_{\mu}(X)$ in $\mathrm{M}_{\mu}$.

Proof. We have $f=\iota_{\mu}(u)$ for a certain element $u \in \mathrm{P}(U)$, which has weight $\nu$ under the adjoint action of $\mathfrak{t}$ on $\mathrm{P}(U)$. In Subsection 2.1 we equipped the algebra $\mathrm{H}(U)$ with $\mathbb{Z}$-grading so that the elements of $U$ and $U^{*}$ have degrees 1 and -1 , respectively. Since the weights of the elements $X, \varepsilon(Y)$ and $u$ of $\mathrm{H}(U)$ are the same, by the condition (6) these elements also have the same (nonnegative) degree. Thus

$$
\operatorname{deg} X=-\operatorname{deg} Y=\operatorname{deg} u .
$$

Consider the element $(X Y) \diamond f$ of $\mathrm{M}_{\mu}$. It corresponds to the product $X Y P u$ in $\overline{\mathrm{A}}$. Write the extremal projector $P$ as a sum, as we did at the beginning of the proof of Proposition 2.3. Then move the generators $F_{\alpha}$ and $E_{\alpha}$ of $\mathfrak{g}$, respectively, to the left of $X Y$ and to the right of $u$ in the resulting summands of $X Y P u$. This procedure shows that modulo $\overline{\mathrm{J}}+\overline{\mathrm{J}}^{\prime}$, the product $X Y P u$ equals the sum of products of the form $X^{\prime} Y^{\prime} u^{\prime}$, where

$$
X^{\prime} \in \mathrm{P}(U)^{\nu-\alpha}, \quad Y^{\prime} \in \mathrm{P}\left(U^{*}\right)^{-\nu-\beta}, \quad \text { and } \quad u^{\prime} \in \mathrm{P}(U)^{\nu+\alpha+\beta},
$$

while $\alpha, \beta$ are certain sums of positive roots.

Suppose that the coset of $X^{\prime} Y^{\prime} u^{\prime}$ makes a nonzero contribution to $(X Y) \diamond f \in$ $\mathrm{M}_{\mu}$. The condition (5) on a Howe system implies that the adjoint operator $\operatorname{ad}_{\zeta\left(F_{\alpha}\right)}$ on $\mathrm{H}(U)$ either has zero degree, or vanishes on the subspace $\mathrm{P}(U)$. Hence

$$
\operatorname{deg} X^{\prime}=\operatorname{deg} X \text {. }
$$

Thus the element $Y^{\prime} u^{\prime} \in \mathrm{H}(U)$ has the degree zero. By our assumption, this element does not belong to the left ideal $\mathrm{H}(U) U^{*} \subset \mathrm{H}(U)$. It also has a weight relative to the adjoint action of $\mathfrak{t}$ on $\mathrm{H}(U)$. But then the weight must be zero. Indeed, by the condition (8) on a Howe system, the adjoint action of $\mathfrak{t}$ on $\mathrm{H}(U)$ preserves the left ideal $\mathrm{H}(U) U^{*}$. Modulo this ideal, the element $Y^{\prime} u^{\prime}$ of $\mathrm{H}(U)$ equals a nonzero scalar, which has weight zero. Thus the weight of $Y^{\prime} u^{\prime}$ is zero as well. Therefore $\alpha=0$ and $X^{\prime}=X$.

The element $Y \diamond f$ of $\mathrm{M}_{\mu}$ corresponds to the product $Y P u$ in $\overline{\mathrm{A}}$. Modulo $\overline{\mathrm{J}}+\overline{\mathrm{J}}^{\prime}$, the latter product equals the sum of the products $Y^{\prime} u^{\prime} \in \mathrm{H}(U)$, where $Y^{\prime}$ and $u^{\prime}$ are the same as above in the particular case when $\alpha=0$ :

$$
Y^{\prime} \in \mathrm{P}\left(U^{*}\right)^{-\nu-\beta} \text { and } \quad u^{\prime} \in \mathrm{P}(U)^{\nu+\beta},
$$


where $\beta$ ranges over sums of positive roots. Modulo the left ideal $\mathrm{H}(U) U^{*}$ of $\mathrm{H}(U)$, the sum of the products $Y^{\prime} u^{\prime}$ equals a scalar. This scalar must be 1 because $Y \diamond f=\iota_{\mu}(1)$ in $\mathrm{M}_{\mu}$. Hence the sum of the products $X Y^{\prime} u^{\prime}$ equals $X$ modulo $\mathrm{H}(U) U^{*} \subset \mathrm{H}(U)$.

The contravariance of $S_{\mu}^{\lambda}$ implies that the kernel Ker $S_{\mu}^{\lambda}$ is an S-submodule of $\mathrm{M}_{\mu}^{\lambda}$. Indeed, let $f \in \operatorname{Ker} S_{\mu}^{\lambda}$ and $Z \in \mathrm{S}$. Then $Z \diamond f \in \operatorname{Ker} S_{\mu}^{\lambda}$, since for $g \in \mathrm{M}_{\mu}^{\lambda}$

$$
S_{\mu}^{\lambda}(Z \diamond f, g)=S_{\mu}^{\lambda}(f, \varepsilon(Z) \diamond g)=0 .
$$

Proposition 2.6. Suppose the weight $\lambda+\rho \in \mathfrak{t}^{*}$ is dominant. Then the quotient space $\mathrm{M}_{\mu}^{\lambda} / \operatorname{Ker} S_{\mu}^{\lambda}$ is an irreducible S-module.

Proof. Take any $f \in \mathrm{M}_{\mu}^{\lambda}$ such that $f \notin \operatorname{Ker} S_{\mu}^{\lambda}$, so that the image of $f$ in the quotient space $\mathrm{M}_{\mu}^{\lambda} / \operatorname{Ker} S_{\mu}^{\lambda}$ is not zero. For any $Y \in \mathrm{P}\left(U^{*}\right)^{-\nu}$ consider the vector $Y \diamond f \in \mathrm{M}_{\mu}$. This vector is well defined because the denominators of $P=P[\rho]$ do not vanish on $f$. The weight of this vector is $\lambda-\nu=\mu+\kappa$, while the subspace $\mathrm{M}_{\mu}^{\mu+\kappa} \subset \mathrm{M}_{\mu}$ is spanned by the vector $\iota_{\mu}(1)$. Hence $Y \diamond f$ is a multiple of $\iota_{\mu}(1)$.

Suppose that $Y \diamond f=0$ for every $Y \in \mathrm{P}\left(U^{*}\right)^{-\nu}$. The map $\varepsilon$ provides a bijection $\mathrm{P}\left(U^{*}\right)^{-\nu} \rightarrow \mathrm{P}(U)^{\nu}$ of subspaces of $\mathrm{H}(U)$. Hence for any $g \in \mathrm{M}_{\mu}^{\lambda}$, there is an element $Y \in \mathrm{P}\left(U^{*}\right)^{-\nu}$, such that $g=\iota_{\mu}(\varepsilon(Y))$. If the weight $\mu+\kappa$ is generic, then

$$
S_{\mu}^{\lambda}(f, g)=S_{\mu}\left(f, \varepsilon(Y) \diamond \iota_{\mu}(1)\right)=S_{\mu}\left(Y \diamond f, \iota_{\mu}(1)\right)=0 .
$$

By our definition of the form $S_{\mu}^{\lambda}$, then we must have $S_{\mu}^{\lambda}(f, g)=0$ for all weights $\mu$, not only those where $\mu+\kappa$ is generic. Hence $f \in \operatorname{Ker} S_{\mu}^{\lambda}$, a contradiction. It shows that there is $Y \in \mathrm{P}\left(U^{*}\right)^{-\nu}$ such that $Y \diamond f \neq 0$. Moreover, we can choose $Y$ with $Y \diamond f=\iota_{\mu}(1)$.

Now let us take any $h \in \mathrm{M}_{\mu}^{\lambda}$ and choose $X \in \mathrm{P}(U)^{\nu}$ such that $\iota_{\mu}(X)=h$. By Lemma 2.5, we then have $(X Y) \diamond f=h$. Here $X Y$ stands for the coset in $\overline{\mathrm{Z}}$ of the product of $X \in \mathrm{P}(U)^{\nu}$ and $Y \in \mathrm{P}\left(U^{*}\right)^{-\nu}$ in the algebra A. This coset belongs to $\mathrm{S}$ by definition because the product of $X$ and $Y$ in A has weight zero.

Recall that $\mathrm{Q}$ is a subalgebra of S. By Proposition [1.6 we now get the following

Corollary 2.7. Suppose that $\lambda+\rho \in \mathfrak{t}^{*}$ is dominant and that $\mathfrak{R}_{\lambda}=\mathfrak{S}_{\lambda}$. Then the quotient space $\mathrm{M}_{\mu}^{\lambda} / \operatorname{Ker} S_{\mu}^{\lambda}$ is an irreducible $\mathrm{Q}$-module.

2.5. Irreducible Q-modules. In this subsection we give another construction of irreducible Q-modules, which employs Proposition [1.9. For any weight $\mu \in \mathfrak{t}^{*}$ denote by $L_{\mu}$ the quotient of the Verma module $M_{\mu}$ by its maximal proper submodule $N_{\mu}$. This quotient is an irreducible $\mathfrak{g}$-module. Note that $\mathrm{P}(U)$ is irreducible as a module over the algebra $\mathrm{H}(U)$. Hence the tensor product $L_{\mu} \otimes \mathrm{P}(U)$ is an irreducible module over the algebra $\mathrm{A}=\mathrm{U}(\mathfrak{g}) \otimes \mathrm{H}(U)$. Recall that the action of the algebra $\mathrm{H}(U)$ on the vector space $\mathrm{P}(U)$ is defined by (2.15).

We can also regard $L_{\mu} \otimes \mathrm{P}(U)$ as a $\mathfrak{g}$-module, by restriction from A to its subalgebra $\mathrm{U}(\mathfrak{g})$. Here $\mathfrak{g}$ acts on the tensor factor $\mathrm{P}(U)$ via the homomorphism $\zeta: \mathrm{U}(\mathfrak{g}) \rightarrow \mathrm{H}(U)$. Let us show that then the actions on $\mathrm{P}(U)$ of the subalgebras $\mathfrak{t}$ and $\mathfrak{n}^{\prime}$ of $\mathfrak{g}$ are semisimple and locally nilpotent, respectively. Indeed, the action of the group $\mathrm{G}$ on $\mathrm{H}(U)$ is locally finite; see Subsection 2.1. Hence the action of $\mathfrak{g}$ on $\mathrm{H}(U)$ adjoint to $\zeta$ is also locally finite, by the property (2) of a Howe system. In particular, the actions of $\mathfrak{t}$ and $\mathfrak{n}^{\prime}$ on $\mathrm{H}(U)$ adjoint to $\zeta$ are semisimple and locally 
nilpotent, respectively. So too are the actions of $\mathfrak{t}$ and $\mathfrak{n}^{\prime}$ on $\mathrm{P}(U)$, by (2.15) and the property (7) of a Howe system. It follows that the actions of $\mathfrak{t}$ and $\mathfrak{n}^{\prime}$ on $L_{\mu} \otimes \mathrm{P}(U)$ are also semisimple and locally nilpotent, respectively.

Take any $\lambda \in \mathfrak{t}^{*}$ such that $\lambda+\rho$ is dominant. Consider the space of $\mathfrak{n}$-coinvariants of the $\mathfrak{g}$-module $L_{\mu} \otimes \mathrm{P}(U)$, and denote by $\mathrm{L}_{\mu}^{\lambda}$ its weight subspace

$$
\left(L_{\mu} \otimes \mathrm{P}(U)\right)_{\mathfrak{n}}^{\lambda} \subset\left(L_{\mu} \otimes \mathrm{P}(U)\right)_{\mathfrak{n}} .
$$

Then $\mathrm{L}_{\mu}^{\lambda}$ is an irreducible S-module by Proposition 1.9. Using Proposition 1.6 we get

Corollary 2.8. Suppose that $\lambda+\rho \in \mathfrak{t}^{*}$ is dominant and that $\mathfrak{R}_{\lambda}=\mathfrak{S}_{\lambda}$. Then $\mathrm{L}_{\mu}^{\lambda}$ is an irreducible Q-module.

Let us now regard $\mathrm{L}_{\mu}^{\lambda}$ as the quotient of the vector subspace $\mathrm{M}_{\mu}^{\lambda}$ by its subspace

$$
\mathrm{N}_{\mu}^{\lambda}=\left(N_{\mu} \otimes \mathrm{P}(U)\right)_{\mathfrak{n}}^{\lambda} .
$$

We will prove that under certain conditions on $\lambda$ and $\mu$, the subspace $\mathrm{N}_{\mu}^{\lambda}$ of $\mathrm{M}_{\mu}^{\lambda}$ coincides with the kernel Ker $S_{\mu}^{\lambda}$ of the Shapovalov form on $\mathrm{M}_{\mu}^{\lambda}$. Then the quotient $\mathrm{L}_{\mu}^{\lambda}$ of $\mathrm{M}_{\mu}^{\lambda}$ coincides with $\mathrm{M}_{\mu}^{\lambda} / \operatorname{Ker} S_{\mu}^{\lambda}$. In particular, these two quotients are then the same as Q-modules. Observe that for any vector $u \in \mathrm{P}(U)$ of the weight $\lambda-\mu$, the denominators of $\zeta(P[\mu+\rho]) u$ get evaluated as products of factors of the form

$$
(\lambda-\mu)\left(H_{\alpha}\right)+(\mu+\rho)\left(H_{\alpha}\right)+s=(\lambda+\rho)\left(H_{\alpha}\right)+s,
$$

where $\alpha \in \Delta^{+}$and $s$ is a positive integer. These factors do not depend on $\mu$ and they do not vanish when the weight $\lambda+\rho$ is dominant.

Note that here the weight $\lambda-\mu$ of the vector $u \in \mathrm{P}(U)$ is taken relative to the action of $\mathfrak{g}$ on $\mathrm{P}(U)$ via the homomorphism $\zeta: \mathrm{U}(\mathfrak{g}) \rightarrow \mathrm{H}(U)$ by using the definition (2.15). If we used the adjoint action of $\mathfrak{g}$ on $\mathrm{P}(U)$ as on a subalgebra of $\mathrm{H}(U) \subset \mathrm{A}$, as we did in the previous subsection, then the weight of the same vector $u$ would be equal to (2.18).

Proposition 2.9. Suppose that $\lambda+\rho \in \mathfrak{t}^{*}$ is dominant and that $\zeta(P[\mu+\rho]) u \neq 0$ for some vector $u \in \mathrm{P}(U)$ of weight $\lambda-\mu$. Then

$$
\mathrm{N}_{\mu}^{\lambda}=\operatorname{Ker} S_{\mu}^{\lambda} .
$$

Proof. Consider the vector $1_{\mu} \otimes u \in M_{\mu} \otimes \mathrm{P}(U)$. This vector is of weight $\lambda$. We will show that the image $\iota_{\mu}(u)$ of this vector in the quotient space

$$
\mathrm{M}_{\mu}^{\lambda}=\left(M_{\mu} \otimes \mathrm{P}(U)\right)_{\mathfrak{n}}^{\lambda}
$$

does not belong to the sum $\mathrm{N}_{\mu}^{\lambda}+\operatorname{Ker} S_{\mu}^{\lambda}$. This implies the equality (2.33). Indeed, then

$$
\mathrm{M}_{\mu}^{\lambda} /\left(\mathrm{N}_{\mu}^{\lambda}+\operatorname{Ker} S_{\mu}^{\lambda}\right)
$$

is a nonzero quotient of

$$
\mathrm{M}_{\mu}^{\lambda} / \mathrm{N}_{\mu}^{\lambda}=\mathrm{L}_{\mu}^{\lambda},
$$

which is an irreducible S-module by Proposition 1.9. So the quotients (2.34) and (2.35) of $\mathrm{M}_{\mu}^{\lambda}$ must coincide, and we obtain the inclusion $\operatorname{Ker} S_{\mu}^{\lambda} \subset \mathrm{N}_{\mu}^{\lambda}$. The opposite inclusion is obtained from the irreducibility of $\mathrm{M}_{\mu}^{\lambda} / \operatorname{Ker} S_{\mu}^{\lambda}$ as an S-module; see Proposition 2.6.

Now assume that $\iota_{\mu}(u) \in \mathrm{N}_{\mu}^{\lambda}+\operatorname{Ker} S_{\mu}^{\lambda}$. We shall bring this to a contradiction. By our assumption, the element $\iota_{\mu}(u) \in \mathrm{M}_{\mu}^{\lambda}$ equals the sum of an element of (2.32) 
and of an element $\iota_{\mu}(v) \in \operatorname{Ker} S_{\mu}^{\lambda}$, where $v \in \mathrm{P}(U)$. Regard $\mathrm{M}_{\mu}^{\lambda}$ as a $\mathfrak{g}$-module, and apply the extremal projector $P=P[\rho]$ to that equality. Then we get a certain equality in the vector space $M_{\mu} \otimes \mathrm{P}(U)$. Here we use the second property in (1.6).

By applying $P$ to $\iota_{\mu}(u) \in \mathrm{M}_{\mu}^{\lambda}$, we get the vector $P\left(1_{\mu} \otimes u\right) \in M_{\mu} \otimes \mathrm{P}(U)$, which belongs to

$$
1_{\mu} \otimes \zeta(P[\mu+\rho]) u+\left(\mathfrak{n} M_{\mu}\right) \otimes \mathrm{P}(U) .
$$

Note that here $\zeta(P[\mu+\rho]) u \neq 0$. By applying $P$ to any element (2.32) we get a vector from the subspace

$$
P\left(N_{\mu} \otimes \mathrm{P}(U)\right) \subset N_{\mu} \otimes \mathrm{P}(U) \subset\left(\mathfrak{n} M_{\mu}\right) \otimes \mathrm{P}(U) .
$$

By applying $P$ to the element $\iota_{\mu}(v) \in \mathrm{M}_{\mu}^{\lambda}$ we get the vector $P\left(1_{\mu} \otimes v\right) \in M_{\mu} \otimes \mathrm{P}(U)$, which belongs to

$$
1_{\mu} \otimes \zeta(P[\mu+\rho]) v+\left(\mathfrak{n} M_{\mu}\right) \otimes \mathrm{P}(U) .
$$

But since $\iota_{\mu}(v) \in \operatorname{Ker} S_{\mu}^{\lambda}$, here we have $\zeta(P[\mu+\rho]) v=0$ by Proposition 2.3. We use the nondegeneracy of the restriction of the form $\langle$,$\rangle to any weight subspace of$ $\mathrm{P}(U)$, proved at the very end of Subsection 2.3. Thus we get a contradiction.

Later on we will produce vectors $u$ satisfying the conditions of Proposition 2.9 for each example of a Howe system and for certain weights $\mu$; see Propositions 3.5, 4.4, and 4.6.

\section{INTERTWINING OPERATORS}

3.1. Zhelobenko operators and left ideals. The vector space $W=U \oplus U^{*}$ has been equipped with a nondegenerate bilinear form $B$, symmetric or alternating. For each isotropic subspace $V \subset W$ denote by $\mathrm{I}_{V}$ the left ideal of the algebra A generated by the elements of $1 \otimes V$. Also consider the left ideal

$$
\overline{\mathrm{I}}_{V}=\overline{\mathrm{U}(\mathfrak{t})} \mathrm{I}_{V}
$$

of algebra $\overline{\mathrm{A}}$. It is generated by the elements of the subspace $1 \otimes V \subset \overline{\mathrm{A}}$. In particular,

$$
\mathrm{I}_{U^{*}}=\mathrm{I} \text { and } \overline{\mathrm{I}}_{U^{*}}=\overline{\mathrm{I}} \text {. }
$$

In Subsection 2.1 we assumed that the group $\mathrm{G}$ acts on the vector space $W$ and preserves the form $B$. So for any $\sigma \in \mathfrak{S}$, the subspace $\widehat{\sigma}(V) \subset W$ is isotropic.

Lemma 3.1. For any element $\sigma \in \mathfrak{S}$ the operator $\check{\xi}_{\sigma}$ on $\overline{\mathrm{A}} / \overline{\mathrm{J}}$ maps

$$
(\overline{\mathrm{I}}+\overline{\mathrm{J}}) / \overline{\mathrm{J}} \rightarrow\left(\overline{\mathrm{I}}_{\widehat{\sigma}\left(U^{*}\right)}+\overline{\mathrm{J}}\right) / \overline{\mathrm{J}} .
$$

Proof. We use induction on the length $\ell(\sigma)$ of the element $\sigma \in \mathfrak{S}$. For $\ell(\sigma)=0$, the statement to prove is tautological. Take any index $c \in\{1, \ldots, r\}$ with $\ell\left(\sigma_{c} \sigma\right)=$ $\ell(\sigma)+1$. Then $\check{\xi}_{\sigma_{c} \sigma}=\check{\xi}_{c} \check{\xi}_{\sigma}$. Note that here we have $\sigma^{-1}\left(\alpha_{c}\right) \in \Delta^{+}$. Take any element $X \in \overline{\mathrm{I}}$. By the induction assumption, the operator $\check{\xi}_{\sigma}$ maps the coset of $X$ in $\overline{\mathrm{A}} / \overline{\mathrm{J}}$ to that of

$$
\sum_{k=1}^{n} Y_{k} \cdot 1 \otimes \widehat{\sigma}\left(u_{k}^{\prime}\right)
$$

where $u_{1}^{\prime}, \ldots, u_{n}^{\prime}$ are basis elements of $U^{*}$ and $Y_{1}, \ldots, Y_{n}$ are certain elements of $\overline{\mathrm{A}}$. By definition, the operator $\check{\xi}_{c}$ maps the latter coset to

$$
\sum_{k=1}^{n} \bar{\xi}_{c}\left(\widehat{\sigma}_{c}\left(Y_{k}\right) \cdot 1 \otimes \widehat{\sigma}_{c} \widehat{\sigma}\left(u_{k}^{\prime}\right)\right) .
$$


By the definition (1.14), to make the induction step it now suffices to show that

$$
\left[F_{c}, 1 \otimes \widehat{\sigma}_{c} \widehat{\sigma}\left(U^{*}\right)\right] \subset 1 \otimes \widehat{\sigma}_{c} \widehat{\sigma}\left(U^{*}\right) .
$$

Applying to this relation the automorphism $\left(\widehat{\sigma}_{c} \widehat{\sigma}\right)^{-1}$ of $\overline{\mathrm{A}}$, we obtain the relation

$$
\left[E_{\alpha}, 1 \otimes U^{*}\right] \subset 1 \otimes U^{*} \quad \text { for } \quad \alpha=\sigma^{-1}\left(\alpha_{c}\right) \in \Delta^{+},
$$

which holds by the condition (8). Here $\left(\widehat{\sigma}_{c} \widehat{\sigma}\right)^{-1}\left(F_{c}\right)$ is a multiple of $E_{\alpha}$ because the action of $\operatorname{Norm}_{0} \mathrm{~T}$ on the subspace $\mathfrak{t} \subset \mathfrak{g}$ factors through that of $\mathfrak{S}$.

In Subsection 2.3 for any $\mu \in \mathfrak{t}^{*}$, we introduced the left ideals $J_{\mu}^{\prime \prime}$ and $\bar{J}_{\mu}^{\prime \prime}$ of the algebras A and $\bar{A}$, respectively. Generalizing (2.14), we introduce the quotient spaces

$$
\mathrm{A}_{\mu, V}=\mathrm{A} /\left(\mathrm{J}_{\mu}^{\prime \prime}+\mathrm{I}_{V}\right) \quad \text { and } \quad \mathrm{M}_{\mu, V}=\mathrm{A}_{\mu, V} / \mathrm{J}=\mathrm{A} /\left(\mathrm{J}_{\mu}^{\prime \prime}+\mathrm{I}_{V}+\mathrm{J}\right)
$$

of $\mathrm{A}$. Then $\mathrm{A}_{\mu, V}$ is a left $\mathrm{A}$-module with the space of $\mathfrak{n}$-coinvariants $\mathrm{M}_{\mu, V}$. Note that for any element $\tau \in \mathfrak{T}$ the action of its representative $\widehat{\tau} \in \mathrm{G}$ on the algebra $\mathrm{A}$ determines a linear map

$$
\mathrm{M}_{\mu, V} \rightarrow \mathrm{M}_{\tau(\mu), \widehat{\tau}(V)} .
$$

Indeed, the adjoint action of $\widehat{\tau}$ on $\mathfrak{g}$ preserves the subalgebras $\mathfrak{n}$ and $\mathfrak{n}^{\prime}$. The action of $\widehat{\tau}$ on the algebra A preserves the right ideal J, and maps the left ideals $\mathrm{J}_{\mu}^{\prime \prime}$ and $\mathrm{I}_{V}$ to $\mathrm{J}_{\tau(\mu)}^{\prime \prime}$ and $\mathrm{I}_{\widehat{\tau}(V)}$, respectively. The linear map (3.2) determined in this way is clearly invertible.

If the weight $\mu+\kappa$ is generic, then the spaces of double cosets

$$
\overline{\mathrm{A}} /\left(\overline{\mathrm{J}}_{\mu}^{\prime \prime}+\overline{\mathrm{I}}_{V}+\overline{\mathrm{J}}\right) \text { and } \mathrm{A} /\left(\mathrm{J}_{\mu}^{\prime \prime}+\mathrm{I}_{V}+\mathrm{J}\right)=\mathrm{M}_{\mu, V}
$$

are naturally isomorphic. Here we use the equality (2.13). Furthermore, then $\mathrm{M}_{\mu, V}$ is isomorphic to the left $\overline{\mathrm{Z}}$-module, defined as the quotient of the algebra $\overline{\mathrm{Z}}$ by the left ideal generated by the cosets of the elements of $1 \otimes V$ and the cosets of the elements $H-(\mu+\kappa)(H)$, where $H \in \mathfrak{t}$. Now denote by $\mathrm{J}^{\prime \prime}$ and $\overline{\mathrm{J}}^{\prime \prime}$ the left ideals of $\mathrm{A}$ and $\overline{\mathrm{A}}$, respectively, generated by the elements $X \otimes 1$ with $X \in \mathfrak{n}^{\prime}$.

Lemma 3.2. For any element $\sigma \in \mathfrak{S}$ the operator $\check{\xi}_{\sigma}$ on $\overline{\mathrm{A}} / \overline{\mathrm{J}}$ maps

$$
\left(\overline{\mathrm{J}}^{\prime \prime}+\overline{\mathrm{I}}+\overline{\mathrm{J}}\right) / \overline{\mathrm{J}} \rightarrow\left(\overline{\mathrm{J}}^{\prime \prime}+\overline{\mathrm{I}}_{\widehat{\sigma}\left(U^{*}\right)}+\overline{\mathrm{J}}\right) / \overline{\mathrm{J}} .
$$

Proof. For each $c=1, \ldots, r$ denote by $\mathfrak{n}_{c}^{\prime}$ the vector subspace of $\mathfrak{n}^{\prime}$ spanned by all root vectors $E_{\alpha}$ except $E_{c}$. Denote by $\mathrm{J}_{c}^{\prime \prime}$ the left ideal of A generated by all elements $X \otimes 1$ with $X \in \mathfrak{n}_{c}^{\prime}$, and by the element $E_{c}$. Let $\overline{\mathrm{J}}_{c}^{\prime \prime}=\overline{\mathrm{U}(\mathfrak{t})} \mathrm{J}_{c}$ be the corresponding left ideal of $\overline{\mathrm{A}}$. We will first prove that the operator $\check{\xi}_{c}$ on the vector space $\overline{\mathrm{A}} / \overline{\mathrm{J}}$ maps

$$
\left(\overline{\mathrm{J}}_{c}^{\prime \prime}+\overline{\mathrm{J}}\right) / \overline{\mathrm{J}} \rightarrow\left(\overline{\mathrm{J}}^{\prime \prime}+\overline{\mathrm{J}}\right) / \overline{\mathrm{J}} .
$$

The left ideal $\widehat{\sigma}_{c}\left(\bar{J}_{c}^{\prime \prime}\right)$ is generated by the element $F_{c}$, and by the subspace of $\bar{A}$ formed by all elements $X \otimes 1$, where $X \in \mathfrak{n}_{c}^{\prime}$. Observe that the latter subspace is preserved by the adjoint action of the element $F_{c}$. Hence for any element $Y \in \overline{\mathrm{A}}$ and any element $Z$ from that subspace, $\bar{\xi}_{c}(Y Z) \in \overline{\mathrm{J}}^{\prime \prime}+\overline{\mathrm{J}}$ by the definition of the operator $\bar{\xi}_{c}$. The property (3.3) of the operator $\check{\xi}_{c}$ on $\bar{A} / \overline{\mathrm{J}}$ now follows from the inclusion $\bar{\xi}_{c}\left(Y F_{c}\right) \in \overline{\mathrm{J}}$ for every $Y \in \overline{\mathrm{A}}$. For a detailed proof of that inclusion, see [KO, Section 7.2] or [KN1, Section 3].

We will now prove Lemma 3.2 by induction on the length $\ell(\sigma)$ of the element $\sigma \in \mathfrak{S}$. For $\ell(\sigma)=0$ the statement to prove is tautological. Take any $c \in\{1, \ldots, r\}$ 
with $\ell\left(\sigma_{c} \sigma\right)=\ell(\sigma)+1$. Then $\check{\xi}_{\sigma_{c} \sigma}=\check{\xi}_{c} \check{\xi}_{\sigma}$. Note that here we have $\sigma^{-1}\left(\alpha_{c}\right) \in \Delta^{+}$. Take the statement of Lemma 3.2 as the induction assumption. Observe an equality of left ideals

$$
\overline{\mathrm{J}}^{\prime \prime}+\overline{\mathrm{I}}_{\widehat{\sigma}\left(U^{*}\right)}=\overline{\mathrm{J}}_{c}^{\prime \prime}+\overline{\mathrm{I}}_{\widehat{\sigma}\left(U^{*}\right)}
$$

of $\bar{A}$. Indeed, by the definitions of left ideals $\bar{J}^{\prime \prime}$ and $\bar{J}_{c}^{\prime \prime}$ the equality (3.4) follows from the inclusion

$$
1 \otimes \zeta\left(E_{c}\right) \in \overline{\mathrm{I}}_{\widehat{\sigma}\left(U^{*}\right)} .
$$

Using the automorphism $\widehat{\sigma}^{-1}$ of the algebra $\overline{\mathrm{A}}$, the latter inclusion is equivalent to

$$
1 \otimes \zeta\left(E_{\alpha}\right) \in \overline{\mathrm{I}} \quad \text { for } \quad \alpha=\sigma^{-1}\left(\alpha_{c}\right) \in \Delta^{+},
$$

which holds by the condition (7) on a Howe system. To make the induction step, it now suffices to prove that the operator $\check{\xi}_{c}$ on $\overline{\mathrm{A}} / \overline{\mathrm{J}}$ maps

$$
\left(\overline{\mathrm{J}}_{c}^{\prime \prime}+\overline{\mathrm{I}}_{\widehat{\sigma}\left(U^{*}\right)}+\overline{\mathrm{J}}\right) / \overline{\mathrm{J}} \rightarrow\left(\overline{\mathrm{J}}^{\prime \prime}+\overline{\mathrm{I}}_{\widehat{\sigma}_{c} \widehat{\sigma}\left(U^{*}\right)}+\overline{\mathrm{J}}\right) / \overline{\mathrm{J}} .
$$

But that follows from the property (3.3) of the operator $\check{\xi}_{c}$, and from the induction step we made when proving Lemma 3.1.

Proposition 3.3. Let $\mu+\kappa \in \mathfrak{t}^{*}$ be generic. Then the operator $\check{\xi}_{\sigma}$ on $\overline{\mathrm{A}} / \overline{\mathrm{J}}$ maps

$$
\left(\overline{\mathrm{J}}_{\mu}^{\prime \prime}+\overline{\mathrm{I}}+\overline{\mathrm{J}}\right) / \overline{\mathrm{J}} \rightarrow\left(\overline{\mathrm{J}}_{\sigma \circ \mu}^{\prime \prime}+\overline{\mathrm{I}}_{\widehat{\sigma}\left(U^{*}\right)}+\overline{\mathrm{J}}\right) / \overline{\mathrm{J}} .
$$

Proof. The left ideal $\overline{\mathrm{J}}_{\mu}^{\prime \prime}$ of the algebra $\overline{\mathrm{A}}$ is spanned by $\overline{\mathrm{J}}^{\prime \prime}$ and by all the subspaces

$$
\overline{\mathrm{A}}(H \otimes 1-\mu(H)),
$$

where $H \in \mathfrak{t}$. Due to Lemma 3.2, to prove Proposition 3.3 it suffices to consider the action of $\check{\xi}_{\sigma}$ on the images of the subspaces (3.5) in the quotient $\bar{A} / \bar{J}$. Write (3.5) as

$$
\overline{\mathrm{A}}(H-(\mu+\kappa)(H)-1 \otimes \zeta(H)+\kappa(H)) .
$$

By (1.17) the operator $\check{\xi}_{\sigma}$ maps the image of $\overline{\mathrm{A}} H$ in $\overline{\mathrm{A}} / \overline{\mathrm{J}}$ to that of $\overline{\mathrm{A}}(\sigma \circ H)$. By (2.12) we have

$$
1 \otimes \zeta(H)-\kappa(H) \in \overline{\mathrm{I}} .
$$

So by Lemma 3.1, the image of (3.6) in $\overline{\mathrm{A}} / \overline{\mathrm{J}}$ is mapped by $\check{\xi}_{\sigma}$ to a subspace of the image in $\overline{\mathrm{A}} / \overline{\mathrm{J}}$ of

$$
\overline{\mathrm{A}}(\sigma \circ H-(\mu+\kappa)(H))+\overline{\mathrm{I}}_{\widehat{\sigma}\left(U^{*}\right)} .
$$

We will complete the proof of Proposition 3.3 by showing that for every $H \in \mathfrak{t}^{*}$,

$$
\sigma \circ H-(\mu+\kappa)(H) \in \overline{\mathrm{J}}_{\sigma \circ \mu}^{\prime \prime}+\overline{\mathrm{I}}_{\widehat{\sigma}\left(U^{*}\right)} .
$$

Replacing $H$ by $\sigma^{-1} \circ H$ on the left-hand side of (3.8), we get the element

$$
H-(\sigma \circ(\mu+\kappa))(H)=H \otimes 1-(\sigma \circ \mu)(H)+1 \otimes \zeta(H)-\sigma(\kappa)(H) .
$$

By definition, here

$$
H \otimes 1-(\sigma \circ \mu)(H) \in \overline{\mathrm{J}}_{\sigma \circ \mu}^{\prime \prime} .
$$

But by applying to the relation (3.7) the automorphism $\widehat{\sigma}$ of $\overline{\mathrm{A}}$, using the condition (1) on a Howe system and then replacing $H$ by $\sigma^{-1}(H)$ in the resulting relation, we get

$$
1 \otimes \zeta(H)-\sigma(\kappa)(H) \in \mathrm{I}_{\widehat{\sigma}\left(U^{*}\right)} .
$$


Corollary 3.4. Let $\mu+\kappa \in \mathfrak{t}^{*}$ be generic. Then for any $\sigma \in \mathfrak{S}$, the operator $\check{\xi}_{\sigma}$ defines a $\overline{\mathrm{Z}}$-equivariant linear map

$$
\mathrm{M}_{\mu} \rightarrow \mathrm{M}_{\sigma \circ \mu, \widehat{\sigma}\left(U^{*}\right)} .
$$

Denote this linear map by the same symbol $\check{\xi}_{\sigma}$. Then its $\overline{\mathrm{Z}}$-equivariance means

$$
\check{\xi}_{\sigma}(Z \diamond f)=\check{\xi}_{\sigma}(Z) \diamond \check{\xi}_{\sigma}(f) \text { for } Z \in \overline{\mathrm{Z}} \quad \text { and } f \in \mathrm{M}_{\mu} \text {. }
$$

This property follows from Proposition 1.4(iii). Note that by (1.16), the abovedefined operator (3.9) maps the weight subspace $\mathrm{M}_{\mu}^{\lambda} \subset \mathrm{M}_{\mu}$ to the weight subspace

$$
\mathrm{M}_{\sigma \circ \mu, \widehat{\sigma}\left(U^{*}\right)}^{\sigma \circ \lambda} \subset \mathrm{M}_{\sigma \circ \mu, \widehat{\sigma}\left(U^{*}\right)} .
$$

3.2. Action of Zhelobenko operators. For any element $\sigma \in \mathfrak{S}$ and any weight $\mu \in \mathfrak{t}^{*}$, consider the A-module

$$
\mathrm{A}_{\sigma \circ \mu, \widehat{\sigma}\left(U^{*}\right)}=\mathrm{A} /\left(\mathrm{J}_{\sigma \circ \mu}^{\prime \prime}+\mathrm{I}_{\widehat{\sigma}\left(U^{*}\right)}\right) .
$$

Its vector space can be identified with that of the tensor product of the Verma module $M_{\sigma \circ \mu}$ over $\mathfrak{g}$, multiplied by the $\mathrm{H}(U)$-module $\mathrm{H}(U) / \mathrm{H}(U) \widehat{\sigma}\left(U^{*}\right)$. The latter quotient space can be identified with $\mathrm{P}(\widehat{\sigma}(U))$. The vector space of (3.10) is then identified with $M_{\sigma \circ \mu} \otimes \mathrm{P}(\widehat{\sigma}(U))$. Further, the space $\mathrm{M}_{\sigma \circ \mu, \widehat{\sigma}\left(U^{*}\right)}$ of $\mathfrak{n}$ coinvariants of (3.10) can be identified with $\mathrm{P}(\widehat{\sigma}(U))$, by assigning to any $w \in$ $\mathrm{P}(\widehat{\sigma}(U))$ the coset of $1 \otimes w \in \mathrm{A}$ in

$$
\mathrm{M}_{\sigma \circ \mu, \widehat{\sigma}\left(U^{*}\right)}=\mathrm{A} /\left(\mathrm{J}_{\sigma \circ \mu}^{\prime \prime}+\mathrm{I}_{\widehat{\sigma}\left(U^{*}\right)}+\mathrm{J}\right) .
$$

In particular, when $\sigma \in \mathfrak{S}$ is the identity element, the space $\mathrm{M}_{\mu, U^{*}}=\mathrm{M}_{\mu}$ gets identified with $\mathrm{P}(U)$, as in Subsection 2.3.

Suppose that the weight $\mu+\kappa$ is generic. Using Corollary 3.4 together with the above identifications of the source and target vector spaces in (3.9), the operator $\check{\xi}_{\sigma}$ on $\overline{\mathrm{A}} / \overline{\mathrm{J}}$ determines a linear map $\mathrm{P}(U) \rightarrow \mathrm{P}(\widehat{\sigma}(U))$. The latter map will be denoted by $I_{\sigma, \mu}$.

Proposition 3.5. Let $\mu+\kappa \in \mathfrak{t}^{*}$ be generic. Then for any $\sigma \in \mathfrak{S}$ and any $u \in \mathrm{P}(U)$,

$$
I_{\sigma, \mu}(u)=\widehat{\sigma}\left(\zeta\left(P_{\sigma}[\mu+\rho]\right) u\right),
$$

where $\zeta\left(P_{\sigma}[\mu+\rho]\right) u$ is regarded as an element of $\mathrm{P}(U)$ by using (2.15).

Proof. We prove Proposition 3.5 by induction on the length $\ell(\sigma)$ of $\sigma \in \mathfrak{S}$. When $\sigma$ is the identity element of $\mathfrak{S}$, the statement to prove is trivial. Let us now use the statement as the induction assumption. Take any index $c \in\{1, \ldots, r\}$ with $\ell\left(\sigma_{c} \sigma\right)=\ell(\sigma)+1$. Then $\sigma^{-1}\left(\alpha_{c}\right)$ is a positive root we denote by $\alpha$. For short, denote by $v$ the element $\zeta\left(P_{\sigma}[\mu+\rho]\right) u \in \mathrm{P}(U)$. We have

$$
\mathrm{M}_{\sigma_{c} \sigma \circ \mu, \widehat{\sigma}_{c} \widehat{\sigma}\left(U^{*}\right)}=\overline{\mathrm{A}} /\left(\overline{\mathrm{J}}_{\sigma_{c} \sigma \circ \mu}^{\prime \prime}+\overline{\mathrm{I}}_{\sigma_{c} \sigma\left(U^{*}\right)}+\overline{\mathrm{J}}\right) \text {. }
$$

Using the definition (1.14) together with the induction assumption, the element $I_{\sigma_{c} \sigma, \mu}(u)$ of $\mathrm{P}(\widehat{\sigma}(U))$ can be identified with the coset in (3.13) of the sum

$$
\sum_{s=0}^{\infty} \prod_{t=1}^{s}\left(t\left(H_{c}-t+1\right)\right)^{-1} \cdot E_{c}^{s} \operatorname{ad}_{F_{c}}^{s}\left(1 \otimes \widehat{\sigma}_{c} \widehat{\sigma}(v)\right) .
$$

Without changing the sum, we can replace the operator $\operatorname{ad}_{F_{c}}^{s}$ by $1 \otimes \operatorname{ad}_{\zeta\left(F_{c}\right)}^{s}$ here because the elements $F_{c} \otimes 1$ and $1 \otimes \widehat{\sigma}_{c} \widehat{\sigma}(v)$ of $\overline{\mathrm{A}}$ commute. Without changing 
the coset, we can then replace $E_{c}^{s}$ by $1 \otimes \zeta\left(E_{c}^{s}\right)$ in resulting sum because $E_{c} \otimes 1$ commutes with

$$
1 \otimes \operatorname{ad}_{\zeta\left(F_{c}\right)}^{s}\left(\widehat{\sigma}_{c} \widehat{\sigma}(v)\right)
$$

and belongs to the left ideal $\mathrm{J}^{\prime \prime} \subset \mathrm{J}_{\sigma_{c} \sigma \circ \mu}^{\prime \prime}$. So we get the sum

$$
\begin{aligned}
& \sum_{s=0}^{\infty} \prod_{t=1}^{s}\left(t\left(H_{c}-t+1\right)\right)^{-1} \cdot 1 \otimes \zeta\left(E_{c}^{s}\right) \operatorname{ad}_{\zeta\left(F_{c}\right)}^{s}\left(\widehat{\sigma}_{c} \widehat{\sigma}(v)\right) \\
& =\sum_{s=0}^{\infty} \prod_{t=1}^{s}\left(t\left(H_{c}-t+1\right)\right)^{-1} \cdot 1 \otimes \widehat{\sigma}_{c} \widehat{\sigma}\left(\zeta\left(F_{\alpha}^{s}\right) \operatorname{ad}_{\zeta\left(E_{\alpha}\right)}^{s}(v)\right) .
\end{aligned}
$$

By the property $(7)$ of a Howe system, the element $1 \otimes \zeta\left(E_{\alpha}\right)$ is in the left ideal $\mathrm{I}=\mathrm{I}_{U^{*}}$ of A. Hence

$$
1 \otimes \widehat{\sigma}_{c} \widehat{\sigma}\left(\zeta\left(E_{\alpha}\right)\right) \in \mathrm{I}_{\sigma_{c} \sigma\left(U^{*}\right)} .
$$

Therefore in the last displayed sum, the element $\operatorname{ad}_{\zeta\left(E_{\alpha}\right)}^{s}(v) \in \mathrm{H}(U)$ can be replaced by the element $\zeta\left(E_{\alpha}^{s}\right) v \in \mathrm{H}(U)$, without changing the coset of the sum in (3.13). We get

$$
\sum_{s=0}^{\infty} \prod_{t=1}^{s}\left(t\left(H_{c}-t+1\right)\right)^{-1} \cdot 1 \otimes \widehat{\sigma}_{c} \widehat{\sigma}\left(\zeta\left(F_{\alpha}^{s}\right) \zeta\left(E_{\alpha}^{s}\right) v\right) .
$$

Here $H_{c}$ stands for the sum $H_{c} \otimes 1+1 \otimes \zeta\left(H_{c}\right)$. Modulo the left ideal $\mathrm{J}_{\sigma_{c} \sigma \circ \mu}^{\prime \prime}$ of A, the element $H_{c} \otimes 1$ equals

$$
\begin{gathered}
\left(\sigma_{c} \sigma \circ \mu\right)\left(H_{c}\right)=\left(\sigma_{c} \sigma(\mu+\rho)-\rho\right)\left(H_{c}\right) \\
=(\mu+\rho)\left(\left(\sigma_{c} \sigma\right)^{-1}\left(H_{c}\right)\right)-\rho\left(H_{c}\right)=-(\mu+\rho)\left(H_{\alpha}\right)-1 .
\end{gathered}
$$

Hence the coset of the sum (3.14) in (3.13) coincides with that of

$$
\begin{aligned}
& \sum_{s=0}^{\infty} \prod_{t=1}^{s} 1 \otimes\left(t\left(\zeta\left(H_{c}\right)-(\mu+\rho)\left(H_{\alpha}\right)-t\right)\right)^{-1} \cdot 1 \otimes \widehat{\sigma}_{c} \widehat{\sigma}\left(\zeta\left(F_{\alpha}^{s}\right) \zeta\left(E_{\alpha}^{s}\right) v\right) \\
= & \sum_{s=0}^{\infty} 1 \otimes \widehat{\sigma}_{c} \widehat{\sigma}\left(\prod_{t=1}^{s}\left(t\left(-\zeta\left(H_{\alpha}\right)-(\mu+\rho)\left(H_{\alpha}\right)-t\right)\right)^{-1} \cdot \zeta\left(F_{\alpha}^{s}\right) \zeta\left(E_{\alpha}^{s}\right) v\right) \\
& =1 \otimes \widehat{\sigma}_{c} \widehat{\sigma}\left(\zeta\left(P_{\alpha}[\mu+\rho]\right) v\right)=1 \otimes \widehat{\sigma}_{c} \widehat{\sigma}\left(\zeta\left(P_{\sigma_{c} \sigma}[\mu+\rho]\right) u\right) .
\end{aligned}
$$

The operator (3.9) has been defined only when the weight $\mu+\kappa$ is generic. However, Proposition 3.5 yields the following result, which is valid for any $\mu \in \mathfrak{t}^{*}$. Here we also use the remark made at the very end of Subsection 3.1.

Corollary 3.6. If $\lambda+\rho$ is dominant, the operator $\check{\xi}_{\sigma}$ on $\overline{\mathrm{A}} / \overline{\mathrm{J}}$ defines a linear map

$$
\mathrm{M}_{\mu}^{\lambda} \rightarrow \mathrm{M}_{\sigma \circ \mu, \widehat{\sigma}\left(U^{*}\right)}^{\sigma \circ \lambda} .
$$

Proof. The source and target spaces in (3.9) can be identified with $\mathrm{P}(U)$ and $\mathrm{P}(\widehat{\sigma}(U))$, respectively. The first of these identifications uses the bijection $\iota_{\mu}$ as in Subsection 2.3. Let $u \in \mathrm{P}(U)$ be any element of weight $\lambda-\mu$ relative to the action of $\mathfrak{t}$. Here any element $H \in \mathfrak{t}$ acts on $\mathrm{P}(U)$ via the left multiplication by $\zeta(H)$, using (2.15). Then $\iota_{\mu}(u) \in \mathrm{M}_{\mu}^{\lambda}$. When applying the operator $\zeta\left(P_{\sigma}[\mu+\rho]\right)$ to $u$, the denominators become, up to nonzero scalar multipliers, certain products of the factors of the form

$$
(\lambda-\mu)\left(H_{\alpha}\right)+(\mu+\rho)\left(H_{\alpha}\right)+s=(\lambda+\rho)\left(H_{\alpha}\right)+s,
$$


where $\alpha$ is a positive root and $s$ is a positive integer. These factors do not depend on $\mu$, and do not vanish if $\lambda+\rho$ is dominant. Thus by mapping $u$ to the element of $\mathrm{P}(\widehat{\sigma}(U))$ on the right-hand side of (3.12), we get the required linear map (3.15).

3.3. Irreducibility theorem. Take the longest element $\sigma_{0}$ of the Weyl group $\mathfrak{S}$. For any weight $\mu \in \mathfrak{t}^{*}$ consider the $\mathrm{R}$-module $\mathrm{M}_{\sigma_{0} \circ \mu, \widehat{\sigma}_{0}\left(U^{*}\right)}$. Next Proposition 3.7 establishes a connection between the Shapovalov form $S_{\mu}^{\lambda}$ on $\mathrm{M}_{\mu}^{\lambda}$, and the linear map

$$
\mathrm{M}_{\mu}^{\lambda} \rightarrow \mathrm{M}_{\sigma_{0} \circ \mu, \widehat{\sigma}_{0}\left(U^{*}\right)}^{\sigma_{0} \circ \lambda}
$$

defined by the Zhelobenko operator $\check{\xi}_{0}=\check{\xi}_{\sigma_{0}}$ on $\overline{\mathrm{A}} / \overline{\mathrm{J}}$, when $\lambda+\rho \in \mathfrak{t}^{*}$ is dominant. The latter map will be denoted by $\check{\xi}_{0} \mid \mathrm{M}_{\mu}^{\lambda}$. Note that when $\mu+\kappa \in \mathfrak{t}^{*}$ fails to be generic, the operator $\check{\xi}_{0}$ on $\overline{\mathrm{A}} / \overline{\mathrm{J}}$ does not necessarily define any map from the whole space $\mathrm{M}_{\mu}$. By combining Proposition 3.7 with Corollary 2.7, we will obtain Theorem 3.8 .

Proposition 3.7. Let $\lambda+\rho \in \mathfrak{t}^{*}$ be dominant. Then for any $\mu \in \mathfrak{t}^{*}$

$$
\operatorname{Ker} S_{\mu}^{\lambda}=\operatorname{Ker}\left(\check{\xi}_{0} \mid \mathrm{M}_{\mu}^{\lambda}\right) \text {. }
$$

Proof. According to Subsection 3.2, the vector space of the R-module $\mathrm{M}_{\sigma_{0} \circ \mu, \widehat{\sigma}_{0}\left(U^{*}\right)}$ can be identified with $\mathrm{P}\left(\widehat{\sigma}_{0}(U)\right)$. Denote by $\langle,\rangle_{\sigma_{0}}$ the nondegenerate $\mathbb{C}$-bilinear pairing

defined by the equality

$$
\mathrm{P}(U) \times \mathrm{P}\left(\widehat{\sigma}_{0}(U)\right) \rightarrow \mathbb{C}
$$

Here $\langle$,$\rangle is the nondegenerate symmetric bilinear form on the vector space U$ selected in Subsection 2.1. Hence the pairing $\langle,\rangle_{\sigma_{0}}$ is nondegenerate also.

Now for any $u, v \in \mathrm{P}(U)$, put $f=\iota_{\mu}(u)$ and $g=\iota_{\mu}(v)$. If the weight $\mu+\kappa$ is generic, then by Propositions 2.3 and 3.5 we have

$$
S_{\mu}^{\lambda}(f, g)=\langle u, \zeta(P[\mu+\rho]) v\rangle=\left\langle u, \widehat{\sigma}_{0}^{-1}\left(I_{\sigma_{0}, \mu}(v)\right)\right\rangle=\left\langle u, I_{\sigma_{0}, \mu}(v)\right\rangle_{\sigma_{0}} .
$$

When the difference $\lambda-\mu$ and the elements $u, v \in \mathrm{P}(U)$ are fixed, the left-hand side of the above equalities becomes a rational function of $\mu \in \mathfrak{t}^{*}$. If $\lambda+\rho$ is dominant, this rational function has finite values for all $\mu$ by Proposition 2.4. Now Proposition 3.7 follows from the definition of the operator $\breve{\xi}_{0} \mid \mathrm{M}_{\mu}^{\lambda}$; see our proof of Corollary 3.6 .

Theorem 3.8. Suppose that $\lambda+\rho \in \mathfrak{t}^{*}$ is dominant and that $\mathfrak{R}_{\lambda}=\mathfrak{S}_{\lambda}$. Then for any $\mu \in \mathfrak{t}^{*}$ the quotient $\mathrm{M}_{\mu}^{\lambda} / \operatorname{Ker}\left(\check{\xi}_{0} \mid \mathrm{M}_{\mu}^{\lambda}\right)$ is an irreducible Q-module.

Recall that the algebra $\mathrm{A}$ is specified as the tensor product (2.5), where the group $\mathrm{G}$ acts diagonally. Consider the corresponding subalgebra $\mathrm{A}^{\mathrm{G}} \subset \mathrm{A}$ of $\mathrm{G}$-invariants. This subalgebra acts on $A_{\mu}$ by restricting the action of $A$. Since $A^{G} \subset$ Norm J, the subalgebra $A^{G}$ then acts on the space $M_{\mu}$ of $\mathfrak{n}$-coinvariants of $A_{\mu}$. The latter action preserves the subspace $\mathrm{M}_{\mu}^{\lambda} \subset \mathrm{M}_{\mu}$. This subspace is also a Q-module, if the weight $\lambda+\rho$ is dominant. The above action of $\mathrm{A}^{\mathrm{G}}$ on $\mathrm{M}_{\mu}^{\lambda}$ can also be obtained by pulling the action of $\mathrm{Q}$ back through the isomorphism $\gamma: \mathrm{A}^{\mathrm{G}} \rightarrow \mathrm{Q}$; see Subsection 1.5. Note that for any $\sigma \in \mathfrak{S}$ the actions of $\mathrm{A}^{\mathrm{G}}$ on the source and target vector spaces of the map (3.15) defined by $\check{\xi}_{\sigma}$ are intertwined by this map by its definition. Using this observation when $\sigma=\sigma_{0}$, we obtain a corollary to Theorem 3.8 . 
Corollary 3.9. Suppose that $\lambda+\rho \in \mathfrak{t}^{*}$ is dominant and that $\mathfrak{R}_{\lambda}=\mathfrak{S}_{\lambda}$. Then for any $\mu \in \mathfrak{t}^{*}$ the quotient $\mathrm{M}_{\mu}^{\lambda} / \operatorname{Ker}\left(\check{\xi}_{0} \mid \mathrm{M}_{\mu}^{\lambda}\right)$ is an irreducible $\mathrm{A}^{\mathrm{G}}$-module.

3.4. Contravariant pairing. For any $\mu \in \mathfrak{t}^{*}$ we can define a nondegenerate $\mathbb{C}$ bilinear pairing

$$
Q_{\mu}: \mathrm{M}_{\mu} \times \mathrm{M}_{\sigma_{0} \circ \mu, \widehat{\sigma}_{0}\left(U^{*}\right)} \rightarrow \mathbb{C}
$$

as follows. For any two elements $u \in \mathrm{P}(U)$ and $w \in \mathrm{P}\left(\widehat{\sigma}_{0}(U)\right)$ consider the cosets in $\mathrm{M}_{\mu}$ and $\mathrm{M}_{\sigma_{0} \circ \mu, \widehat{\sigma}_{0}\left(U^{*}\right)}$ of $1 \otimes u$ and $1 \otimes w$, respectively; see (3.11). By definition, the value of $Q_{\mu}$ on this pair of cosets is $\langle u, w\rangle_{\sigma_{0}}$; see (3.17). By restricting $Q_{\mu}$ to weight subspaces, we define a pairing

$$
Q_{\mu}^{\lambda}: \mathrm{M}_{\mu}^{\lambda} \times \mathrm{M}_{\sigma_{0} \circ \mu, \widehat{\sigma}_{0}\left(U^{*}\right)}^{\sigma_{0} \circ \lambda} \rightarrow \mathbb{C}
$$

for any $\lambda \in \mathfrak{t}^{*}$. The latter pairing is also nondegenerate; see the end of Subsection 2.3.

Now suppose that the weight $\lambda+\rho$ is dominant. Let us prove that then

$$
Q_{\mu}^{\lambda}(\varepsilon(Z) \diamond f, h)=Q_{\mu}^{\lambda}\left(f, \check{\xi}_{0}(Z) \diamond h\right) \quad \text { when } \quad Z \in \mathrm{S} .
$$

In particular, here $\check{\xi}_{0}(Z) \diamond h \in \mathrm{M}_{\sigma_{0} \circ \mu, \widehat{\sigma}_{0}\left(U^{*}\right)}^{\sigma_{0} \circ \lambda}$ is defined, even though the element $\check{\xi}_{0}(Z)$ of the algebra $\bar{Z}$ may not be contained in the subspace $\mathrm{Z} \subset \overline{\mathrm{Z}}$.

First let $\mu+\kappa$ be generic. Then the map (3.16) defined by the Zhelobenko operator $\check{\xi}_{0}$ on $\overline{\mathrm{A}} / \overline{\mathrm{J}}$ is invertible. Let $g \in \mathrm{M}_{\mu}^{\lambda}$ be the image of $h$ under the inverse map. By (3.18)

$$
Q_{\mu}^{\lambda}(\varepsilon(Z) \diamond f, h)=S_{\mu}^{\lambda}(\varepsilon(Z) \diamond f, g)=S_{\mu}^{\lambda}(f, Z \diamond g)=Q_{\mu}^{\lambda}\left(f, \check{\xi}_{0}(Z) \diamond h\right) .
$$

Here we used (2.30) and Corollary 3.4. Thus we get (3.20) for generic $\mu+\kappa$. Now note that when $\lambda+\rho$ is dominant, the left-hand side of (3.20) is defined for any weight $\mu$, as a finite number. When $f, h, Z$ and the difference $\lambda-\mu$ are all fixed while $\mu$ varies, this number becomes a rational function of $\mu$. Hence the right-hand side of (3.20) can also be defined as a rational function of the weight $\mu$, with only finite values. Since the pairing (3.19) is nondegenerate, we can then determine the vector $\check{\xi}_{0}(Z) \diamond h$.

If $Z \in \mathrm{Q}$, then $\check{\xi}_{0}(Z)=Z$ by definition. Hence the equality (3.20) implies that for any $\mu$ and dominant $\lambda+\rho$ the pairing (3.19) is Q-contravariant:

$$
Q_{\mu}^{\lambda}(\varepsilon(Z) \diamond f, h)=Q_{\mu}^{\lambda}(f, Z \diamond h) \quad \text { when } \quad Z \in \mathrm{Q} .
$$

In particular, we have an action $\diamond$ of the algebra $\mathrm{Q}$ on the vector space $\mathrm{M}_{\sigma_{0} \circ \mu, \widehat{\sigma}_{0}\left(U^{*}\right)}^{\sigma_{0} \circ \lambda}$.

\section{YANGians AND REDUCTIVE DUAL PAIRS}

4.1. Reductive dual pairs. From now on we will work with examples of Howe systems. For each of the corresponding algebras (2.5) we will describe explicitly its subalgebra of G-invariants. Then we will apply Corollary 3.9 to each of these examples. The group $\mathrm{G}$ will be one of the classical complex Lie groups $\mathrm{GL}_{m}, \mathrm{Sp}_{2 m}$, and $\mathrm{O}_{2 m}$ with any positive integer $m$, and $\mathfrak{g}$ will be one of the Lie algebras $\mathfrak{g l}_{m}$, $\mathfrak{s p}_{2 m}$, and $\mathfrak{s o}_{2 m}$, respectively.

First consider $\mathrm{G}=\mathrm{GL}_{m}$. Let the indices $a$ and $b$ run through $1, \ldots, m$. Then $e_{a}$ will denote a vector of the standard basis in the vector space $\mathbb{C}^{m}$, while $E_{a b} \in \mathfrak{g l}_{m}$ will be a standard matrix unit. Choose the standard triangular decomposition (1.1) of $\mathfrak{g}=\mathfrak{g l}_{m}$ where the subalgebras $\mathfrak{n}, \mathfrak{t}, \mathfrak{n}^{\prime}$ are spanned by the matrix units $E_{a b}$ with $a>b, a=b, a<b$, respectively. The elements $E_{a a}$ form a basis in $\mathfrak{t}$, and we will 
denote by $\eta_{a}$ the vector of the dual basis in $\mathfrak{t}^{*}$ corresponding to $E_{a a}$. The positive and negative roots are $\eta_{a}-\eta_{b}$ with $a<b$ and $a>b$, respectively. The semisimple rank $r$ of $\mathfrak{g l}_{m}$ is $m-1$. For each $c=1, \ldots, m-1$, we will choose $\eta_{c}-\eta_{c+1}$ as the simple root $\alpha_{c}$, and

$$
H_{c}=E_{c c}-E_{c+1, c+1}, \quad E_{c}=E_{c, c+1}, \quad \text { and } \quad F_{c}=E_{c+1, c}
$$

as the basis elements of the $\mathfrak{s l}_{2}$-subalgebra $\mathfrak{g}_{c} \subset \mathfrak{g}$. Then define the Chevalley anti-involution $\varepsilon$ on $\mathfrak{g l}_{m}$ by setting $\varepsilon\left(E_{a b}\right)=E_{b a}$, and choose the trivial Cartan decomposition $\mathfrak{g}=\mathfrak{g}_{+} \oplus \mathfrak{g}_{-}$of $\mathfrak{g l}_{m}$ so that $\mathfrak{g}_{+}=\mathfrak{g l}_{m}$ and $\mathfrak{g}_{-}=\{0\}$.

The algebraic group $\mathrm{GL}_{m}$ is connected, and the maximal torus $\mathrm{T} \subset \mathrm{GL}_{m}$ with the Lie algebra $\mathfrak{t}$ consists of all elements acting on each basis vector $e_{a} \in \mathbb{C}^{m}$ by a scalar multiplication. The action of $\sigma_{c} \in \mathfrak{S}$ on $\mathfrak{t}^{*}$ exchanges the dual basis vectors $\eta_{c}$ and $\eta_{c+1}$, leaving all other basis vectors fixed. Hence the Weyl group $\mathfrak{S}$ can be identified with the symmetric group $\mathfrak{S}_{m}$. Choose the representative $\widehat{\sigma}_{c} \in$ Norm T so that its action on $\mathbb{C}^{m}$ exchanges the basis vectors $e_{c}$ and $e_{c+1}$, leaving all other basis vectors of $\mathbb{C}^{m}$ fixed. Note that here the group $\mathfrak{T}$ is trivial.

Now consider $\mathrm{G}=\mathrm{Sp}_{2 m}, \mathrm{O}_{2 m}$. Let $a$ and $b$ run through $-m, \ldots,-1,1, \ldots, m$. Then $e_{a}$ will denote a vector of the standard basis in $\mathbb{C}^{2 m}$, and $E_{a b}$ will be a standard matrix unit in $\mathfrak{g l}_{2 m}$. We will regard $\mathrm{G}$ as the subgroup in $\mathrm{GL}_{2 m}$ preserving the bilinear form on $\mathbb{C}^{2 m}$ whose value on any pair $\left(e_{a}, e_{b}\right)$ of the basis vectors is, respectively, $\delta_{a,-b} \cdot \operatorname{sign} a$ or $\delta_{a,-b}$ when $\mathrm{G}$ is $\mathrm{Sp}_{2 m}$ or $\mathrm{O}_{2 m}$. Then $\mathfrak{g}$ is the Lie subalgebra of $\mathfrak{g l}_{2 m}$ spanned by the elements

$$
F_{a b}=E_{a b}-\operatorname{sign} a b \cdot E_{-b,-a} \quad \text { or } \quad F_{a b}=E_{a b}-E_{-b,-a} .
$$

Let us choose the triangular decomposition (1.1) of $\mathfrak{g}=\mathfrak{s p}_{2 m}, \mathfrak{s o}_{2 m}$, where the subalgebras $\mathfrak{n}, \mathfrak{t}, \mathfrak{n}^{\prime}$ are spanned by the elements $F_{a b}$ with $a>b, a=b, a<b$, respectively. The elements $F_{-a,-a}$ with $a>0$ form a basis in $\mathfrak{t}$. Here for any $a>0$ we will denote by $\eta_{a}$ the vector of the dual basis in $\mathfrak{t}^{*}$ corresponding to the basis vector $F_{a-m-1, a-m-1} \in \mathfrak{t}$. The positive roots of $\mathfrak{s p}_{2 m}$ are $\eta_{a}-\eta_{b}$ and $\eta_{a}+\eta_{b}$ where $1 \leqslant a<b \leqslant m$, together with $2 \eta_{a}$ where $1 \leqslant a \leqslant m$. The positive roots of $\mathfrak{s o}_{2 m}$ are only $\eta_{a}-\eta_{b}$ and $\eta_{a}+\eta_{b}$, where $1 \leqslant a<b \leqslant m$. The semisimple rank $r$ of $\mathfrak{s o}_{2 m}$ with $m>1$ and of $\mathfrak{s p}_{2 m}$ is $m$. But the semisimple rank of $\mathfrak{s o}_{2}$ is zero, while the root system is empty.

For $\mathfrak{g}=\mathfrak{s p}_{2 m}, \mathfrak{s o}_{2 m}$ and $c=1, \ldots, m-1$, choose $\alpha_{c}=\eta_{c}-\eta_{c+1}$ and

$H_{c}=F_{c-m-1, c-m-1}-F_{c-m, c-m}, \quad E_{c}=F_{c-m-1, c-m}, \quad$ and $\quad F_{c}=F_{c-m, c-m-1}$.

Further, if $\mathfrak{g}=\mathfrak{s p}_{2 m}$, then choose $\alpha_{m}=2 \eta_{m}$ and

$$
H_{m}=F_{-1,-1}, \quad E_{m}=F_{-1,1} / 2, \quad \text { and } \quad F_{m}=F_{1,-1} / 2 .
$$

If $\mathfrak{g}=\mathfrak{s o}_{2 m}$ and $m>1$, then choose $\alpha_{m}=\eta_{m-1}+\eta_{m}$ and

$$
H_{m}=F_{-2,-2}+F_{-1,-1}, \quad E_{m}=F_{-2,1}, \quad \text { and } \quad F_{m}=F_{1,-2} .
$$

For $\mathfrak{g}=\mathfrak{s p}_{2 m}, \mathfrak{s o}_{2 m}$, choose the Cartan decomposition $\mathfrak{g}=\mathfrak{g}_{+} \oplus \mathfrak{g}_{-}$, where $\mathfrak{g}_{+}$ is spanned by the elements $F_{a b}$ with $a, b>0$ while $\mathfrak{g}_{-}$is spanned by $F_{a,-b}$ and $F_{-a, b}$ with $a, b>0$. The Chevalley anti-involution $\varepsilon$ will be defined by setting $\varepsilon\left(F_{a b}\right)=\operatorname{sign} a b \cdot F_{b a}$ if $\theta=1$, or $\varepsilon\left(F_{a b}\right)=F_{b a}$ if $\theta=-1$. Here the parameter $\theta$ is the same as in Subsection 2.1. This choice of $\varepsilon$ is prescribed by the condition (3) on our particular Howe systems; see below.

The algebraic group $\mathrm{Sp}_{2 m}$ is connected, but $\mathrm{O}_{2 m}$ has two connected components. For $\mathrm{G}=\mathrm{Sp}_{2 m}, \mathrm{O}_{2 m}$ the maximal torus $\mathrm{T} \subset \mathrm{G}_{0}$ with the Lie algebra $\mathfrak{t}$ consists of all 
the elements of $\mathrm{GL}_{2 m}$ which multiply any two basis vectors $e_{a}, e_{-a} \in \mathbb{C}^{2 m}$ by scalars inverse to each other. The Weyl group of $\mathfrak{s p}_{2 m}$ is isomorphic to the semidirect product $\mathfrak{S}_{m} \ltimes \mathbb{Z}_{2}^{m}$, where the symmetric group $\mathfrak{S}_{m}$ acts by permutations of the $m$ copies of $\mathbb{Z}_{2}$. The Weyl group of $\mathfrak{s o}_{2 m}$ is isomorphic to a subgroup of $\mathfrak{S}_{m} \ltimes \mathbb{Z}_{2}^{m}$ of index two. For $c=1, \ldots, m-1$ and $\mathfrak{g}=\mathfrak{s p}_{2 m}, \mathfrak{s o}_{2 m}$ the action of $\sigma_{c} \in \mathfrak{S}$ on $\mathfrak{t}^{*}$ exchanges the basis vectors $\eta_{c}$ and $\eta_{c+1}$, leaving other basis vectors fixed. Then choose the representative $\widehat{\sigma}_{c} \in$ Norm $_{0} \mathrm{~T}$ so that its action on $\mathbb{C}^{2 m}$ exchanges $e_{c-m-1}$ and $e_{c-m}$, also exchanges $e_{m-c+1}$ and $e_{m-c}$, leaving all other basis vectors of $\mathbb{C}^{2 m}$ fixed. For $\mathfrak{g}=\mathfrak{s p}_{2 m}$ we have $\sigma_{m}\left(\eta_{m}\right)=-\eta_{m}$ and $\sigma_{m}\left(\eta_{a}\right)=\eta_{a}$ for $1 \leqslant a<m$. Choose the representative $\widehat{\sigma}_{m} \in \operatorname{Sp}_{2 m}$ so that $\widehat{\sigma}_{m}\left(e_{-1}\right)=e_{1}$ and $\widehat{\sigma}_{m}\left(e_{1}\right)=-e_{-1}$ while $\widehat{\sigma}_{m}\left(e_{a}\right)=e_{a}$ for $|a|>1$. Note that for $\mathfrak{g}=\mathfrak{s p}_{2 m}$ the group $\mathfrak{T}$ is trivial, like it was in the case $\mathfrak{g}=\mathfrak{g l}_{m}$.

Now take $\mathfrak{g}=\mathfrak{s o}_{2 m}$. Here the group $\mathfrak{T}$ is not trivial but is isomorphic to $\mathbb{Z}_{2}$. Let $\tau_{m}$ be the generator of this group. Then $\tau_{m}\left(\eta_{m}\right)=-\eta_{m}$ and $\tau_{m}\left(\eta_{a}\right)=\eta_{a}$ for $1 \leqslant a<m$, so that $\sigma_{m}=\tau_{m} \sigma_{m-1} \tau_{m}$. Choose the representative $\widehat{\tau}_{m} \in \mathrm{O}_{2 m}$ so that $\widehat{\tau}_{m}\left(e_{-1}\right)=e_{1}$ and $\widehat{\tau}_{m}\left(e_{1}\right)=e_{-1}$ while $\widehat{\tau}_{m}\left(e_{a}\right)=e_{a}$ for $|a|>1$. Choose $\widehat{\sigma}_{m} \in \mathrm{O}_{2 m}$ to be $\widehat{\tau}_{m} \widehat{\sigma}_{m-1} \widehat{\tau}_{m}$. We will need a representative in $\mathrm{O}_{2 m}$ for every element $\omega \in \mathfrak{R}$. If $\omega \notin \mathfrak{S}$, then $\omega=\tau_{m} \sigma$ for some $\sigma \in \mathfrak{S}$. In this case, the representative of $\omega$ in $\mathrm{O}_{2 m}$ will be $\widehat{\omega}=\widehat{\tau}_{m} \widehat{\sigma}$.

We will now associate to any $\mathrm{G}$ another classical complex Lie group; it will be denoted by $\mathrm{G}^{\prime}$. Let $n$ be a positive integer. If $\theta=1$, then for $\mathrm{G}=\mathrm{GL}_{m}, \mathrm{Sp}_{2 m}, \mathrm{O}_{2 m}$ put $\mathrm{G}^{\prime}=\mathrm{GL}_{n}, \mathrm{O}_{n}, \mathrm{Sp}_{n}$, respectively. If $\theta=-1$, then put $\mathrm{G}^{\prime}=\mathrm{GL}_{n}, \mathrm{Sp}_{n}, \mathrm{O}_{n}$, respectively. Here for $\mathrm{G}^{\prime}=\mathrm{Sp}_{n}$, the integer $n$ is to be even. Then $\left(\mathrm{G}, \mathrm{G}^{\prime}\right)$ is a reductive dual pair [H1].

Let $\mathfrak{g}^{\prime}$ be the Lie algebra of $\mathrm{G}^{\prime}$. Let the indices $i$ and $j$ run through the sequence $1, \ldots, n$. Then $f_{i}$ will denote a vector of the standard basis in the vector space $\mathbb{C}^{n}$, while $E_{i j} \in \mathfrak{g l}_{n}$ will be a standard matrix unit. If $i$ is even, put $\tilde{\imath}=i-1$. If $i$ is odd and $i<n$, put $\tilde{\imath}=i+1$. Finally, if $i=n$ and $n$ is odd, put $\tilde{\imath}=i$. We will regard $\mathrm{G}^{\prime}=\mathrm{O}_{n}$ or $\mathrm{G}^{\prime}=\mathrm{Sp}_{n}$ as the subgroup in $\mathrm{GL}_{n}$, preserving the bilinear form on $\mathbb{C}^{n}$ whose value on any pair $\left(f_{i}, f_{j}\right)$ of the basis vectors is $\theta_{i} \delta_{\tilde{\imath} j}$, where $\theta_{i}=1$ or $\theta_{i}=(-1)^{i-1}$, respectively. Then $\mathfrak{g}^{\prime}$ is the Lie subalgebra of $\mathfrak{g l}_{n}$ spanned by the elements $E_{i j}-\theta_{i} \theta_{j} E_{\tilde{\jmath} \tau}$.

Let $U$ be the tensor product of vector spaces $\mathbb{C}^{m} \otimes \mathbb{C}^{n}$, and let $U^{*}$ be the dual vector space. For $a=1, \ldots, m$ and $i=1, \ldots, n$, let $x_{a i}$ denote the basis vector $e_{a} \otimes f_{i}$ of $U$. Then let $\partial_{a i}$ denote the corresponding vector of the dual basis in $U^{*}$. If $\theta=1$, then $\mathrm{P}(U)$ is the algebra of polynomials in $x_{a i}$, while $\mathrm{H}(U)$ can be identified with the algebra of differential operators on $\mathrm{P}(U)$, so that $\partial_{a i}$ is the partial derivation corresponding to $x_{a i}$. If $\theta=-1$, then $\mathrm{P}(U)$ is the Grassmann algebra with $m n$ anti-commuting generators $x_{a i}$. The definition (2.15) then implies that the element $x_{a i} \in \mathrm{H}(U)$ acts on $\mathrm{P}(U)$ via left multiplication, while $\partial_{a i} \in \mathrm{H}(U)$ acts as the left derivation relative to $x_{a i}$. The latter operator on the Grassmann algebra is also called the inner multiplication by $x_{a i}$. For any $\theta$ the form $\langle$,$\rangle on$ $U$ will be chosen so that the basis of the $x_{a i}$ is orthonormal. Then the involutive anti-automorphism $\varepsilon$ of the algebra $\mathrm{H}(U)$ exchanges $x_{a i}$ with $\partial_{a i}$.

Let us now consider the vector space $W=U \oplus U^{*}$. The groups $\mathrm{GL}_{m}$ and $\mathrm{GL}_{n}$ act on the vector space $U$, and their actions commute with each other. Hence we get the mutually commuting actions of $\mathrm{GL}_{m}$ and $\mathrm{GL}_{n}$ on $W$. Clearly, both actions preserve the bilinear form $B$ in $W$ as introduced in Subsection 2.1. If $\mathrm{G}=\mathrm{Sp}_{2 m}, \mathrm{O}_{2 m}$, 
then we can identify the vector space $W$ with the tensor product $\mathbb{C}^{2 m} \otimes \mathbb{C}^{n}$ so that for $a=1, \ldots, m$ we have $x_{a i}=e_{a} \otimes f_{i}$ as above, and $\partial_{a i}=e_{-a} \otimes\left(\theta_{i} f_{\tilde{\imath}}\right)$. Then the bilinear form $B$ on $W$ gets identified with the tensor product of the forms on $\mathbb{C}^{2 m}$ and $\mathbb{C}^{n}$ chosen above. If $\theta=1$, then the form $B$ is alternating and $\mathrm{G}^{\prime}=\mathrm{O}_{n}, \mathrm{Sp}_{n}$, respectively. If $\theta=-1$, then the form $B$ is symmetric and $\mathrm{G}^{\prime}=\mathrm{Sp}_{n}, \mathrm{O}_{n}$, respectively. Hence we always obtain mutually commuting actions of $\mathrm{G}$ and $\mathrm{G}^{\prime}$ on the vector space $W$, preserving the bilinear form $B$.

To complete the description of a Howe system on $U$, one requires a homomorphism $\zeta: \mathrm{U}(\mathfrak{g}) \rightarrow \mathrm{H}(U)$ obeying the conditions (1) to (6). It is well known and can be verified directly that $\zeta$ can be chosen so that for $\mathfrak{g}=\mathfrak{g l}_{m}$ and $a, b=1, \ldots, m$

$$
\zeta\left(E_{a b}\right)=\sum_{i=1}^{n} x_{a i} \partial_{b i}
$$

while for $\mathfrak{g}=\mathfrak{s p}_{2 m}, \mathfrak{s o}_{2 m}$ and the same indices $a, b=1, \ldots, m$

$$
\begin{gathered}
\zeta\left(F_{a b}\right)=\theta \delta_{a b} n / 2+\sum_{i=1}^{n} x_{a i} \partial_{b i} \\
\zeta\left(F_{a,-b}\right)=-\sum_{i=1}^{n} \theta \theta_{i} x_{a \tilde{\imath}} x_{b i}, \quad \zeta\left(F_{-a, b}\right)=\sum_{i=1}^{n} \theta_{i} \partial_{a i} \partial_{b \tilde{\imath}} .
\end{gathered}
$$

For $\mathfrak{g}=\mathfrak{g l}_{m}, \mathfrak{s p}_{2 m}, \mathfrak{s o}_{2 m}$, and any $\theta=1,-1$ by [H2, Sections 2.3, 3.5, 3.8, 4.2, 4.3], the image of $\zeta$ coincides with the subalgebra of $\mathrm{G}^{\prime}$-invariant elements in $\mathrm{H}(U)$.

Note that for any $i, j=1, \ldots, n$ the element $E_{i j} \in \mathfrak{g l}_{n}$ acts on $\mathrm{P}(U)$ as the operator

$$
\sum_{a=1}^{m} x_{a i} \partial_{a j}
$$

Hence for $\mathfrak{g}^{\prime}=\mathfrak{s o}_{n}, \mathfrak{s p}_{n}$, the element $E_{i j}-\theta_{i} \theta_{j} E_{\tilde{\jmath} \tilde{\imath}} \in \mathfrak{g}^{\prime}$ acts on $\mathrm{P}(U)$ as the operator

$$
\sum_{a=1}^{m}\left(x_{a i} \partial_{a j}-\theta_{i} \theta_{j} x_{a \tilde{\jmath}} \partial_{a \tilde{\imath}}\right) .
$$

The subalgebra of $\mathrm{G}$-invariants in $\mathrm{H}(U)$ is generated by the elements (4.3) for $\mathrm{G}=\mathrm{GL}_{m}$ or by the elements (4.4) for $\mathrm{G}=\mathrm{Sp}_{2 m}, \mathrm{O}_{2 m}$; see [H1]. In Subsection 4.3 we give an analogue of this result for the algebra (2.5) instead of $\mathrm{H}(U)$.

4.2. Yangians. First consider the Yangian $\mathrm{Y}\left(\mathfrak{g l}_{n}\right)$ corresponding to the Lie algebra $\mathfrak{g}^{\prime}=\mathfrak{g l}_{n}$. This Yangian is a complex unital associative algebra with a family of generators $T_{i j}^{(1)}, T_{i j}^{(2)}, \ldots$ where $i, j=1, \ldots, n$. Defining relations for these generators can be written using the series

$$
T_{i j}(x)=\delta_{i j}+T_{i j}^{(1)} x^{-1}+T_{i j}^{(2)} x^{-2}+\cdots,
$$

where $x$ is a formal parameter. Let $y$ be another formal parameter. Then the defining relations in the associative algebra $\mathrm{Y}\left(\mathfrak{g l}_{n}\right)$ can be written as

$$
(x-y)\left[T_{i j}(x), T_{k l}(y)\right]=T_{k j}(x) T_{i l}(y)-T_{k j}(y) T_{i l}(x) .
$$

The algebra $\mathrm{Y}\left(\mathfrak{g l}_{n}\right)$ is commutative if $n=1$. By (4.5), for any $z \in \mathbb{C}$, the assignments

$$
T_{i j}(x) \mapsto T_{i j}(x+z)
$$


define an automorphism of the algebra $\mathrm{Y}\left(\mathfrak{g l}_{n}\right)$. Here each of the formal power series $T_{i j}(x+z)$ in $(x+z)^{-1}$ should be re-expanded in $x^{-1}$, and every assignment (4.6) is a correspondence between the respective coefficients of series in $x^{-1}$. Relations (4.5) also show that for any formal power series $g(x)$ in $x^{-1}$ with coefficients from $\mathbb{C}$ and leading term 1 , the assignments

$$
T_{i j}(x) \mapsto g(x) T_{i j}(x)
$$

define an automorphism of the algebra $Y\left(\mathfrak{g l}_{n}\right)$. The subalgebra in $Y\left(\mathfrak{g l}_{n}\right)$, consisting of all elements which are invariant under every automorphism of the form (4.7), is the special Yangian of $\mathfrak{g l}_{n}$ and will be denoted by SY $\left(\mathfrak{g l}_{n}\right)$. Two representations of the algebra $\mathrm{Y}\left(\mathfrak{g l}_{n}\right)$ are called similar if they differ by an automorphism of the form (4.7). Similar representations have the same restrictions to the subalgebra $\operatorname{SY}\left(\mathfrak{g l}_{n}\right)$.

Using (4.5), one can check that the assignments

$$
T_{i j}(x) \mapsto \delta_{i j}+E_{i j} x^{-1}
$$

define a homomorphism of unital associative algebras $\mathrm{Y}\left(\mathfrak{g l}_{n}\right) \rightarrow \mathrm{U}\left(\mathfrak{g l}_{n}\right)$. There is also an embedding $\mathrm{U}\left(\mathfrak{g l}_{n}\right) \rightarrow \mathrm{Y}\left(\mathfrak{g l}_{n}\right)$, defined by mapping $E_{i j} \mapsto T_{i j}^{(1)}$. So $\mathrm{Y}\left(\mathfrak{g l}_{n}\right)$ contains the universal enveloping algebra $\mathrm{U}\left(\mathfrak{g l}_{n}\right)$ as a subalgebra. Note that the homomorphism (4.8) is identical on the subalgebra $\mathrm{U}\left(\mathfrak{g l}_{n}\right) \subset \mathrm{Y}\left(\mathfrak{g l}_{n}\right)$.

Let $T(x)$ be the $n \times n$ matrix whose $i, j$ entry is the series $T_{i j}(x)$. One can show that the assignment

$$
T(x) \mapsto T(-x)^{-1}
$$

defines an involutive automorphism of the algebra $\mathrm{Y}\left(\mathfrak{g l}_{n}\right)$. Here each entry of the inverse matrix $T(-x)^{-1}$ is a formal power series in $x^{-1}$ with coefficients from the algebra $\mathrm{Y}\left(\mathfrak{g l}_{n}\right)$ and the assignment (4.9) as a correspondence between the respective matrix entries.

The Yangian $\mathrm{Y}\left(\mathfrak{g l}_{n}\right)$ is a Hopf algebra over the field $\mathbb{C}$. The comultiplication $\Delta: \mathrm{Y}\left(\mathfrak{g l}_{n}\right) \rightarrow \mathrm{Y}\left(\mathfrak{g l}_{n}\right) \otimes \mathrm{Y}\left(\mathfrak{g l}_{n}\right)$ is defined by the assignment

$$
\Delta: T_{i j}(x) \mapsto \sum_{k=1}^{n} T_{i k}(x) \otimes T_{k j}(x) .
$$

When taking tensor products of $\mathrm{Y}\left(\mathfrak{g l}_{n}\right)$-modules, we use the comultiplication (4.10). The counit homomorphism $\mathrm{Y}\left(\mathfrak{g l}_{n}\right) \rightarrow \mathbb{C}$ is defined by the assignment $T_{i j}(x) \mapsto \delta_{i j}$. The antipodal map $\mathrm{Y}\left(\mathfrak{g l}_{n}\right) \rightarrow \mathrm{Y}\left(\mathfrak{g l}_{n}\right)$ is defined by mapping $T(x) \mapsto T(x)^{-1}$. Note that the assignments

$$
T_{i j}(x) \mapsto T_{j i}(x)
$$

define an involutive anti-automorphism of the associative algebra $\mathrm{Y}\left(\mathfrak{g l}_{n}\right)$. Moreover, they define a bialgebra anti-automorphism of $\mathrm{Y}\left(\mathfrak{g l}_{n}\right)$.

The special Yangian $\mathrm{SY}\left(\mathfrak{g l}_{n}\right)$ is a Hopf subalgebra of $\mathrm{Y}\left(\mathfrak{g l}_{n}\right)$. It is isomorphic to the Yangian $\mathrm{Y}\left(\mathfrak{s l}_{n}\right)$ of the special linear Lie algebra $\mathfrak{s l}_{n} \subset \mathfrak{g l}_{n}$ considered in [D1, D2. For the proofs of the latter two assertions, see [M2, Section 1.8].

Now let $\mathfrak{g}^{\prime}$ be one of the two Lie algebras $\mathfrak{s o}_{n}, \mathfrak{s} \mathfrak{p}_{n}$. When considering these two cases simultaneously, we will use the following convention. Whenever the double sign \pm or $\mp$ appears, the upper sign will correspond to the case of a symmetric form on $\mathbb{C}^{n}$ so that $\mathfrak{g}^{\prime}=\mathfrak{s o}_{n}$, while the lower sign will correspond to the case of an alternating form on $\mathbb{C}^{n}$ so that $\mathfrak{g}^{\prime}=\mathfrak{s p}_{n}$. Let $\widetilde{T}(x)$ be the transpose to the matrix $T(x)$ relative to that form on $\mathbb{C}^{n}$. The $i, j$ entry of the matrix $\widetilde{T}(x)$ is $\theta_{i} \theta_{j} T_{\tilde{\jmath} \tilde{\imath}}(x)$; 
see Subsection 4.1. An involutive automorphism of the algebra $\mathrm{Y}\left(\mathfrak{g l}_{n}\right)$ can then be defined by the assignment

$$
T(x) \mapsto \widetilde{T}(-x) .
$$

This assignment is understood as a correspondence between the respective matrix entries. Note that (4.12) defines an anti-automorphism of the coalgebra $\mathrm{Y}\left(\mathfrak{g l}_{n}\right)$, as (4.11) does.

Consider the product $\widetilde{T}(-x) T(x)$. The $i, j$ entry of this matrix is the series

$$
\sum_{k=1}^{n} \theta_{i} \theta_{k} T_{\tilde{k} \tilde{\imath}}(-x) T_{k j}(x) .
$$

The twisted Yangian corresponding to the Lie algebra $\mathfrak{g}^{\prime}$ is the subalgebra of $\mathrm{Y}\left(\mathfrak{g l}_{n}\right)$ generated by coefficients of all series (4.13). We denote this subalgebra by $\mathrm{Y}\left(\mathfrak{g}^{\prime}\right)$. The subalgebra $\mathrm{Y}\left(\mathfrak{g}^{\prime}\right) \cap \mathrm{SY}\left(\mathfrak{g l}_{n}\right)$ of $\mathrm{Y}\left(\mathfrak{g l}_{n}\right)$ will be called the special twisted Yangian corresponding to $\mathfrak{g}^{\prime}$. This subalgebra is denoted by $\operatorname{SY}\left(\mathfrak{g}^{\prime}\right)$. The automorphism (4.7) of $\mathrm{Y}\left(\mathfrak{g l}_{n}\right)$ determines an automorphism of $\mathrm{Y}\left(\mathfrak{g}^{\prime}\right)$ which multiplies the series (4.13) by $g(x) g(-x)$. The subalgebra $\mathrm{SY}\left(\mathfrak{g}^{\prime}\right)$ of $\mathrm{Y}\left(\mathfrak{g}^{\prime}\right)$ consists of the elements fixed by all such automorphisms. Two representations of $\mathrm{Y}\left(\mathfrak{g}^{\prime}\right)$ are called similar if they differ by such an automorphism. Similar representations of $Y\left(\mathfrak{g}^{\prime}\right)$ have the same restriction to the subalgebra $\mathrm{SY}\left(\mathfrak{g}^{\prime}\right)$.

To give defining relations for these generators of $\mathrm{Y}\left(\mathfrak{g}^{\prime}\right)$, let us introduce the extended twisted Yangian $\mathrm{X}\left(\mathfrak{g}^{\prime}\right)$. The complex unital associative algebra $\mathrm{X}\left(\mathfrak{g}^{\prime}\right)$ has a family of generators $S_{i j}^{(1)}, S_{i j}^{(2)}, \ldots$ where $i, j=1, \ldots, n$. Put

$$
S_{i j}(x)=\delta_{i j}+S_{i j}^{(1)} x^{-1}+S_{i j}^{(2)} x^{-2}+\cdots,
$$

and let $S(x)$ be the $n \times n$ matrix whose $i, j$ entry is the series $S_{i j}(x)$. Defining relations in the algebra $\mathrm{X}\left(\mathfrak{g}^{\prime}\right)$ can then be written as

$$
\begin{gathered}
\left(x^{2}-y^{2}\right)\left[S_{i j}(x), S_{k l}(y)\right]=(x+y)\left(S_{k j}(x) S_{i l}(y)-S_{k j}(y) S_{i l}(x)\right) \\
\mp(x-y)\left(\theta_{k} \theta_{j} S_{i \tilde{k}}(x) S_{\tilde{\jmath} l}(y)-\theta_{i} \theta_{l} S_{k \tilde{\imath}}(y) S_{\tilde{l} j}(x)\right) \\
\pm \theta_{i} \theta_{j}\left(S_{k \tilde{\imath}}(x) S_{\tilde{\jmath} l}(y)-S_{k \tilde{\imath}}(y) S_{\tilde{\jmath} l}(x)\right) .
\end{gathered}
$$

They show that for any formal power series $f(x)$ in $x^{-1}$ with coefficients from $\mathbb{C}$ and leading term 1 , an automorphism of the algebra $\mathrm{X}\left(\mathfrak{g}^{\prime}\right)$ is defined by mapping

$$
S_{i j}(x) \mapsto f(x) S_{i j}(x) .
$$

Let $\widetilde{S}(x)$ be the transpose to the matrix $S(x)$ relative to our form on $\mathbb{C}^{n}$, so that the $i, j$ entry of $\widetilde{S}(x)$ is $\theta_{i} \theta_{j} S_{\tilde{\jmath} \tilde{\imath}}(x)$. By [M2, Theorem 2.3.13] there is a formal power series $O(x)$ in $x^{-1}$ with coefficients in the centre of $\mathrm{X}\left(\mathfrak{g}^{\prime}\right)$ and leading term 1 , such that

$$
S(x) \mp 2 x \widetilde{S}(x)=(1 \mp 2 x) O(x) S(-x) .
$$

Moreover, $O(x) O(-x)=1$. Note that (4.16) yields an explicit formula for $O(x)$.

One can define a homomorphism $\mathrm{X}\left(\mathfrak{g}^{\prime}\right) \rightarrow \mathrm{Y}\left(\mathfrak{g}^{\prime}\right)$ by mapping

$$
S(x) \mapsto \widetilde{T}(-x) T(x) .
$$

Moreover, the kernel of the homomorphism (4.17) is generated by the coefficients of the series $O(x)-1$. For the proof of the last two claims, see [M2, Section 2.13]. By 
setting $O(x)=1$ in the matrix relation (4.16) and then considering the $i, j$ entry, we get

$$
S_{i j}(x) \mp 2 x \theta_{i} \theta_{j} S_{\tilde{\jmath} \imath}(x)=(1 \mp 2 x) S_{i j}(-x) .
$$

The homomorphism (4.17) is surjective by its definition. Thus the twisted Yangian $\mathrm{Y}\left(\mathfrak{g}^{\prime}\right)$ can also be defined as the associative unital algebra with the generators $S_{i j}^{(1)}, S_{i j}^{(2)}, \ldots$ subject to the relations (4.2) and (4.18).

The anti-automorphism (4.11) and the automorphism (4.12) of the algebra $\mathrm{Y}\left(\mathfrak{g l}_{n}\right)$ commute with each other. Their composition is an involutive anti-automorphism of $\mathrm{Y}\left(\mathfrak{g l}_{n}\right)$ which maps

$$
T_{i j}(x) \mapsto \theta_{i} \theta_{j} T_{\tilde{\imath} \tilde{\jmath}}(-x) .
$$

The composition (4.19) preserves the subalgebra $\mathrm{Y}\left(\mathfrak{g}^{\prime}\right) \subset \mathrm{Y}\left(\mathfrak{g l}_{n}\right)$. The resulting anti-automorphism of $\mathrm{Y}\left(\mathfrak{g}^{\prime}\right)$ can also be obtained as follows. By using (4.2), one shows that the assignments

$$
S_{i j}(x) \mapsto S_{j i}(x)
$$

define an involutive anti-automorphism of $\mathrm{X}\left(\mathfrak{g}^{\prime}\right)$. Moreover, by (4.18) it factors to anti-automorphism of the algebra $Y\left(\mathfrak{g}^{\prime}\right)$. The latter coincides with the restriction of (4.19) to the subalgebra $\mathrm{Y}\left(\mathfrak{g}^{\prime}\right) \subset \mathrm{Y}\left(\mathfrak{g l}_{n}\right)$.

Suppose we are given a representation of $\mathrm{X}\left(\mathfrak{g}^{\prime}\right)$ such that every coefficient of the series $O(x)$ is represented by a scalar. Denote by $o(x)$ the corresponding series with scalar coefficients. Then $o(x) o(-x)=1$, and the leading term of $o(x)$ is 1 . Hence we can find another formal power series $f(x)$ in $x^{-1}$ with scalar coefficients and the leading term 1 , such that $o(x)=f(-x) / f(x)$. By pulling back the given representation of $\mathrm{X}\left(\mathfrak{g}^{\prime}\right)$ through (4.15), we then get another representation of $\mathrm{X}\left(\mathfrak{g}^{\prime}\right)$, which factors through the homomorphism $\mathrm{X}\left(\mathfrak{g}^{\prime}\right) \rightarrow \mathrm{Y}\left(\mathfrak{g}^{\prime}\right)$. The series $f(x)$ and hence the resulting representation of $\mathrm{Y}\left(\mathfrak{g}^{\prime}\right)$ are not unique. However, here we can only replace $f(x)$ by $f(x) h(x)$ where $h(x)$ is a formal power series in $x^{-1}$ with scalar coefficients and the leading term 1 , such that $h(x)=h(-x)$. Then $h(x)=$ $g(x) g(-x)$ for some series $g(x)$ as in (4.7). Hence all the representations of $\mathrm{Y}\left(\mathfrak{g}^{\prime}\right)$ corresponding to the given representation of $\mathrm{X}\left(\mathfrak{g}^{\prime}\right)$ are similar to each other and have the same restrictions to $\mathrm{SY}\left(\mathfrak{g}^{\prime}\right)$.

The twisted Yangian $\mathrm{Y}\left(\mathfrak{g}^{\prime}\right)$ has an analogue of the homomorphism $\mathrm{Y}\left(\mathfrak{g l}_{n}\right) \rightarrow$ $\mathrm{U}\left(\mathfrak{g l}_{n}\right)$ defined by (4.8). Namely, one can define a homomorphism $\mathrm{X}\left(\mathfrak{g}^{\prime}\right) \rightarrow \mathrm{U}\left(\mathfrak{g}^{\prime}\right)$ by mapping

$$
S_{i j}(x) \mapsto \delta_{i j}+\frac{E_{i j}-\theta_{i} \theta_{j} E_{\tilde{\jmath} \tilde{\imath}}}{x \pm \frac{1}{2}}
$$

This can be proved by using (4.2); see [M2, Proposition 2.1.2]. Moreover, the homomorphism (4.21) factors through the homomorphism $\mathrm{X}\left(\mathfrak{g}^{\prime}\right) \rightarrow \mathrm{Y}\left(\mathfrak{g}^{\prime}\right)$ defined by (4.17). Further, there is an embedding $\mathrm{U}\left(\mathfrak{g}^{\prime}\right) \rightarrow \mathrm{Y}\left(\mathfrak{g}^{\prime}\right)$ defined by mapping each element $E_{i j}-\theta_{i} \theta_{j} E_{\tilde{\jmath} \tau} \in \mathfrak{g}^{\prime}$ to the coefficient at $x^{-1}$ of the series (4.13). Hence $\mathrm{Y}\left(\mathfrak{g}^{\prime}\right)$ contains $\mathrm{U}\left(\mathfrak{g}^{\prime}\right)$ as a subalgebra. The homomorphism $\mathrm{Y}\left(\mathfrak{g}^{\prime}\right) \rightarrow \mathrm{U}\left(\mathfrak{g}^{\prime}\right)$ corresponding to (4.21) is identical on the subalgebra $\mathrm{U}\left(\mathfrak{g}^{\prime}\right) \subset \mathrm{Y}\left(\mathfrak{g}^{\prime}\right)$.

The twisted Yangian $Y\left(\mathfrak{g}^{\prime}\right)$ is not only a subalgebra of $Y\left(\mathfrak{g l}_{n}\right)$, it is also a right coideal of the coalgebra $\mathrm{Y}\left(\mathfrak{g l}_{n}\right)$ relative to the comultiplication (4.10). Indeed, let us apply this comultiplication to the $i, j$ entry of the $n \times n$ matrix $\widetilde{T}(-x) T(x)$. We 
get the sum

$$
\begin{gathered}
\sum_{k=1}^{n} \theta_{i} \theta_{k} \Delta\left(T_{\tilde{k} \tilde{\imath}}(-x) T_{k j}(x)\right) \\
=\sum_{g, h, k=1}^{n} \theta_{i} \theta_{j}\left(T_{\tilde{k} \tilde{g}}(-x) \otimes T_{\tilde{g} \tilde{\imath}}(-x)\right)\left(T_{k h}(x) \otimes T_{h j}(x)\right) \\
=\sum_{g, h, k=1}^{n} \theta_{g} \theta_{k} T_{\tilde{k} \tilde{g}}(-x) T_{k h}(x) \otimes \theta_{i} \theta_{g} T_{\tilde{g} \tilde{\imath}}(-x) T_{h j}(x) .
\end{gathered}
$$

In the last displayed line, by performing the summation over $k=1, \ldots, n$ in the first tensor factor, we get the $g, h$ entry of the matrix $\widetilde{T}(-x) T(x)$. Therefore

$$
\Delta\left(\mathrm{Y}\left(\mathfrak{g}^{\prime}\right)\right) \subset \mathrm{Y}\left(\mathfrak{g}^{\prime}\right) \otimes \mathrm{Y}\left(\mathfrak{g l}_{n}\right) .
$$

For the extended twisted Yangian $\mathrm{X}\left(\mathfrak{g}^{\prime}\right)$, one can define a homomorphism of associative algebras

$$
\mathrm{X}\left(\mathfrak{g}^{\prime}\right) \rightarrow \mathrm{X}\left(\mathfrak{g}^{\prime}\right) \otimes \mathrm{Y}\left(\mathfrak{g l}_{n}\right)
$$

by mapping

$$
S_{i j}(x) \mapsto \sum_{g, h=1}^{n} S_{g h}(x) \otimes \theta_{i} \theta_{g} T_{\tilde{g} \tilde{\imath}}(-x) T_{h j}(x) .
$$

The homomorphism property can be verified directly; see [KN3, Section 3]. Using the homomorphism (4.23), the tensor product of any modules over the algebras $\mathrm{X}\left(\mathfrak{g}^{\prime}\right)$ and $\mathrm{Y}\left(\mathfrak{g l}_{n}\right)$ becomes another module over $\mathrm{X}\left(\mathfrak{g}^{\prime}\right)$.

Furthermore, the homomorphism (4.23) determines a right coaction of the Hopf algebra $\mathrm{Y}\left(\mathfrak{g l}_{n}\right)$ on the algebra $\mathrm{X}\left(\mathfrak{g}^{\prime}\right)$. Formally, one can define a homomorphism of associative algebras

$$
\mathrm{X}\left(\mathfrak{g}^{\prime}\right) \rightarrow \mathrm{X}\left(\mathfrak{g}^{\prime}\right) \otimes \mathrm{Y}\left(\mathfrak{g l}_{n}\right) \otimes \mathrm{Y}\left(\mathfrak{g l}_{n}\right)
$$

in two different ways: either by using the assignment (4.23) twice or by using (4.23) and then (4.10). Both ways lead to the same result; see again [KN3, Section 3].

4.3. Olshanski homomorphisms. First take the pair $\left(\mathrm{G}, \mathrm{G}^{\prime}\right)=\left(\mathrm{GL}_{m}, \mathrm{GL}_{n}\right)$. We will treat the cases $\theta=1$ and $\theta=-1$ simultaneously. We shall use the Yangian $\mathrm{Y}\left(\mathfrak{g l}_{n}\right)$ to describe the subalgebra of $\mathrm{GL}_{m}$-invariant elements in the corresponding algebra (2.5). Denote by $E$ the $m \times m$ matrix whose $a, b$ entry is $E_{b a} \in \mathfrak{g l}_{m}$. Note the transposition of the indices $a$ and $b$ here. The inverse matrix $(x+\theta E)^{-1}$ will be regarded as a formal power series in $x^{-1}$ whose coefficients are certain $m \times m$ matrices with entries from the algebra $\mathrm{U}\left(\mathfrak{g l}_{m}\right)$. Let $(x+\theta E)_{a b}^{-1}$ be the $a, b$ entry of the inverse matrix. This entry equals

$$
\delta_{a b} x^{-1}-\theta E_{b a} x^{-2}+\sum_{s=0}^{\infty} \sum_{c_{1}, \ldots, c_{s}=1}^{m}(-\theta)^{s+1} E_{c_{1} a} E_{c_{2} c_{1}} \cdots E_{c_{s} c_{s-1}} E_{b c_{s}} x^{-s-2} .
$$

The following result first appeared in [01, but in another guise. For connections to our setting, see [KN1, Section 4] or [KN2, Section 4] when $\theta=1$ or $\theta=-1$, respectively. Let $\mathrm{U}\left(\mathfrak{g l}_{m}\right)^{\mathrm{GL}_{m}}$ be the subalgebra of $\mathrm{GL}_{m}$-invariants in $\mathrm{U}\left(\mathfrak{g l}_{m}\right)$; it coincides with the centre of the algebra $\mathrm{U}\left(\mathfrak{g l}_{m}\right)$. 
Proposition 4.1. (i) One can define a homomorphism $\mathrm{Y}\left(\mathfrak{g l}_{n}\right) \rightarrow \mathrm{U}\left(\mathfrak{g l}_{m}\right) \otimes \mathrm{H}(U)$ by

$$
T_{i j}(x) \mapsto \delta_{i j}+\sum_{a, b=1}^{m}(x+\theta m / 2+\theta E)_{a b}^{-1} \otimes x_{a i} \partial_{b j} .
$$

(ii) The subalgebra of $\mathrm{GL}_{m}$-invariant elements in the algebra $\mathrm{A}=\mathrm{U}\left(\mathfrak{g l}_{m}\right) \otimes \mathrm{H}(U)$ coincides with the subalgebra, generated by $\mathrm{U}\left(\mathfrak{g l}_{m}\right)^{\mathrm{GL}_{m}} \otimes 1$ and by the image of the homomorphism (4.24).

Proof. Part (i) was proved in [KN1, Section 1] and [KN2, Section 1] for $\theta=1$ and $\theta=-1$, respectively. The claim that the image of the homomorphism (4.24) belongs to the subalgebra $\mathrm{A}^{\mathrm{GL}_{m}} \subset \mathrm{A}$ was also proved therein. It remains to prove that the elements

$$
\sum_{a=1}^{m} 1 \otimes x_{a i} \partial_{a j}
$$

and

$$
\sum_{a, b, c_{1}, \ldots, c_{s}=1}^{m} E_{c_{1} a} E_{c_{2} c_{1}} \cdots E_{c_{s} c_{s-1}} E_{b c_{s}} \otimes x_{a i} \partial_{b j},
$$

together with $\mathrm{U}\left(\mathfrak{g l}_{m}\right)^{\mathrm{GL}_{m}} \otimes 1$, generate the whole subalgebra $\mathrm{A}^{\mathrm{GL}_{m}} \subset \mathrm{A}$. Here $s=0,1,2, \ldots$ and $i, j=1, \ldots, n$. For $s=0$ the first tensor factor of the summand in (4.26) should be understood as $E_{b a}$.

Let $\mathbb{N}$ be the additive semigroup of nonnegative integers. Take the standard $\mathbb{N}$ filtration on the algebra $\mathrm{U}\left(\mathfrak{g l}_{m}\right)$, where any element of $\mathfrak{g l}_{m}$ has degree 1 . The adjoint action of the group $\mathrm{GL}_{m}$ on $\mathrm{U}\left(\mathfrak{g l}_{m}\right)$ preserves this filtration, and the corresponding graded algebra is identified with the symmetric algebra $\mathrm{S}\left(\mathfrak{g l}_{m}\right)$. The algebra $\mathrm{H}(U)$ has an $\mathbb{N} \times \mathbb{N}$-filtration, such that the elements of $U$ and $U^{*}$ have degrees $(1,0)$ and $(0,1)$, respectively. This filtration is preserved by the action of the group $\mathrm{GL}_{m}$ on $\mathrm{H}(U)$. The corresponding graded algebra is identified with the symmetric algebra of $U \oplus U^{*}$ in the case $\theta=1$, or with the exterior algebra of $U \oplus U^{*}$ in the case $\theta=-1$. In both cases, the graded algebra is denoted by $\mathrm{P}\left(U \oplus U^{*}\right)$. It suffices to prove that the elements of the algebra $\mathrm{S}\left(\mathfrak{g l}_{m}\right) \otimes \mathrm{P}\left(U \oplus U^{*}\right)$ corresponding to (4.25) and (4.26), taken together with

$$
\mathrm{S}\left(\mathfrak{g l}_{m}\right)^{\mathrm{GL}} \mathrm{L}_{m} \otimes 1 \subset \mathrm{S}\left(\mathfrak{g l}_{m}\right) \otimes \mathrm{P}\left(U \oplus U^{*}\right),
$$

generate the whole subalgebra of $\mathrm{GL}_{m}$-invariants. We will prove this for $n=1$ only. Generalization of our proof to any $n \geqslant 1$ will be obvious; cf. [MO, Subsection 2.9].

So let us suppose that $U=\mathbb{C}^{m} \otimes \mathbb{C}^{1}=\mathbb{C}^{m}$. Let $e_{1}^{\prime}, \ldots, e_{m}^{\prime}$ be the basis of the vector space $U^{*}$ dual to the standard basis $e_{1}, \ldots, e_{m}$ of $U$. First consider the $\mathrm{GL}_{m}$-invariants in the subspace

$$
1 \otimes \mathrm{P}\left(U \oplus U^{*}\right) \subset \mathrm{S}\left(\mathfrak{g l}_{m}\right) \otimes \mathrm{P}\left(U \oplus U^{*}\right) .
$$

The subspace of $\mathrm{P}\left(U \oplus U^{*}\right)$ of degree $(p, q)$ is a subspace in $U^{\otimes p} \otimes\left(U^{*}\right)^{\otimes q}$. The latter tensor product contains nonzero $\mathrm{GL}_{m}$-invariant vectors only if $p=q$. The $\mathrm{GL}_{m}$-invariants in $U^{\otimes p} \otimes\left(U^{*}\right)^{\otimes p}$ are arbitrary linear combinations of the sums

$$
\sum_{c_{1}, \ldots, c_{p}=1}^{m} e_{c_{\varpi(1)}} \otimes \cdots \otimes e_{c_{\varpi(p)}} \otimes e_{c_{1}}^{\prime} \otimes \cdots \otimes e_{c_{p}}^{\prime},
$$


where $\varpi$ ranges over all permutations of the indices $1, \ldots, p$. Here we used the classical invariant theory for $\mathrm{GL}_{m}$; see [H1, Theorem $\left.1 \mathrm{~A}\right]$. For $\theta=1$ or $\theta=-1$, respectively, by applying to (4.29) the symmetrization or antisymmetrization in the first $p$ and also in the last $p$ tensor factors of the summand, we get an element of $\mathrm{P}(U) \otimes \mathrm{P}\left(U^{*}\right)=\mathrm{P}\left(U \oplus U^{*}\right)$ corresponding to

$$
\sum_{c_{1}, \ldots, c_{p}=1}^{m} x_{c_{1} 1} \cdots x_{c_{p} 1} \partial_{c_{1} 1} \cdots \partial_{c_{p} 1} \in \mathrm{H}(U)
$$

multiplied by $\theta^{\ell(\varpi)}$. This shows that for $n=1$, the $\mathrm{GL}_{m}$-invariants in the subspace (4.28) are generated by the elements corresponding to (4.25) with $i=j=1$.

Now consider the subspace (4.27). Generators of the subalgebra $\mathrm{S}\left(\mathfrak{g l}_{m}\right)^{\mathrm{GL}_{m}} \subset$ $\mathrm{S}\left(\mathfrak{g l}_{m}\right)$ are well known. We will reproduce a set of generators here because they will be used later on. Identify $\mathfrak{g l}_{m}$ with $U \otimes U^{*}$ as a module of the group $\mathrm{GL}_{m}$, so that the matrix unit $E_{a b} \in \mathfrak{g l}_{m}$ is identified with the vector $e_{a} \otimes e_{b}^{\prime} \in U \otimes U^{*}$. Then the subspace in $\mathrm{S}\left(\mathfrak{g l}_{m}\right)$ of degree $t$ becomes a subspace in $\left(U \otimes U^{*}\right) \otimes t$. The $\mathrm{GL}_{m}$-invariants in the latter tensor product are any linear combinations of the sums

$$
\sum_{c_{1}, \ldots, c_{t}=1}^{m} e_{c_{\varpi(1)}} \otimes e_{c_{1}}^{\prime} \otimes \cdots \otimes e_{c_{\varpi(t)}} \otimes e_{c_{t}}^{\prime},
$$

where $\varpi$ ranges over all permutations of $1, \ldots, t$. Let $s_{1}, \ldots, s_{k}$ be the cycle lengths of the permutation $\varpi$, so that $t=s_{1}+\cdots+s_{k}$. By applying to the sum (4.30) the symmetrization in the $t$ pairs of tensor factors of the summand, we get an element of $\mathrm{S}\left(\mathfrak{g l}_{m}\right)$, which corresponds to the product over $s=s_{1}, \ldots, s_{k}$ of the elements

$$
\sum_{c_{1}, \ldots, c_{s}=1}^{m} E_{c_{2} c_{1}} E_{c_{3} c_{2}} \cdots E_{c_{s} c_{s-1}} E_{c_{1} c_{s}} \in \mathrm{U}\left(\mathfrak{g l}_{m}\right)^{\mathrm{GL}_{m}} .
$$

Now take the subspace in $\mathrm{S}\left(\mathfrak{g l}_{m}\right) \otimes \mathrm{P}\left(U \oplus U^{*}\right)$ of degree $t+1$ in the first tensor factor and of degree $(p, q)$ in the second tensor factor, for any $t \geqslant 0$ and $(p, q) \neq$ $(0,0)$. Regard it as a subspace in the tensor product

$$
\left(U \otimes U^{*}\right)^{\otimes(t+1)} \otimes U^{\otimes p} \otimes\left(U^{*}\right)^{\otimes q} .
$$

The tensor product contains nonzero $\mathrm{GL}_{m}$-invariant vectors, only if $p=q$. Suppose this is the case. Then the $\mathrm{GL}_{m}$-invariants in the tensor product are any linear combinations of the sums over $c_{0}, c_{1}, \ldots, c_{t}, c_{t+1}, \ldots, c_{t+p}=1, \ldots, m$ of the vectors

$$
e_{c_{\varpi(0)}} \otimes e_{c_{0}}^{\prime} \otimes \cdots \otimes e_{c_{\varpi(t)}} \otimes e_{c_{t}}^{\prime} \otimes e_{c_{\varpi(t+1)}} \otimes \cdots \otimes e_{c_{\varpi(t+p)}} \otimes e_{c_{t+1}}^{\prime} \otimes \cdots \otimes e_{c_{t+p}}^{\prime},
$$

where each of the sums corresponds to a permutation $\varpi$ of $0,1, \ldots, t, t+1, \ldots, t+p$. The $\mathrm{GL}_{m}$-invariants in $\mathrm{S}\left(\mathfrak{g l}_{m}\right) \otimes \mathrm{P}\left(U \oplus U^{*}\right)$ are obtained by applying to these linear combinations the symmetrization of the first $t+1$ pairs of factors of the tensor product (4.32), and the symmetrization or anti-symmetrization in the next $p$ and in the last $p$ factors, for $\theta=1$ or $\theta=-1$, respectively. Let $Q_{\varpi}$ be the element of $\mathrm{S}\left(\mathfrak{g l}_{m}\right) \otimes \mathrm{P}\left(U \oplus U^{*}\right)$ obtained by these operations from the sum corresponding to the permutation $\varpi$.

If the cycle of an element of the set $\{0,1, \ldots, t\}$ under the action of the powers of $\varpi$ is contained in the set, then the element $Q_{\varpi} \in \mathrm{S}\left(\mathfrak{g l}_{m}\right) \otimes \mathrm{P}\left(U \oplus U^{*}\right)$ is divisible in the first tensor factor by the element of $\mathrm{S}\left(\mathfrak{g l}_{m}\right)$ corresponding to (4.31), where $s$ is the length of the cycle. Then our argument reduces to a similar one with $t$ 
replaced by $t-s$. Suppose that the $\varpi$-cycle of each element of $\{0,1, \ldots, t\}$ is not contained in this set.

Consider $\varpi(t+1)$. If it belongs to $\{t+1, \ldots, t+p\}$, then $Q_{\varpi}$ is divisible by the element of $\mathrm{S}\left(\mathfrak{g l}_{m}\right) \otimes \mathrm{P}\left(U \oplus U^{*}\right)$ corresponding to (4.25) with $i=j=1$, and our argument reduces to a similar one with $p$ replaced by $p-1$. Suppose that $\varpi(t+1) \in\{0,1, \ldots, t\}$. Due to the symmetrization, without loss of generality we may assume that $\varpi(t+1)=0$. Let $s \geqslant 0$ be the minimal number such that $\varpi^{s+1}(0) \notin\{0,1, \ldots, t\}$. Again due to (anti-)symmetrization, we may assume that

$$
\varpi(0)=1, \varpi(1)=2, \ldots, \varpi(s-1)=s \quad \text { and } \quad \varpi(s)=t+1 .
$$

Now we see that $Q_{\varpi}$ is divisible by the element of $\mathrm{S}\left(\mathfrak{g l}_{m}\right) \otimes \mathrm{P}\left(U \oplus U^{*}\right)$ corresponding to (4.26) with $i=j=1, a=c_{0}$, and $b=c_{t+1}$. This observation reduces our argument to a similar one where $t+1$ and $p$ are replaced by $t-s$ and $p-1$, respectively.

Now let $\left(\mathrm{G}, \mathrm{G}^{\prime}\right)$ be any of the reductive dual pairs from Subsection 4.1 with $\mathrm{G}=\mathrm{Sp}_{2 m}$ or $\mathrm{G}=\mathrm{O}_{2 m}$. Again, we will treat the cases of $\theta=1$ and $\theta=-1$ simultaneously. Denote by $F$ the $2 m \times 2 m$ matrix whose $a, b$ entry is the matrix unit $F_{a b} \in \mathfrak{g}$. Here the indices $a$ and $b$ run through $-m, \ldots,-1,1, \ldots, m$. Regard the inverse matrix $(x-\theta F)^{-1}$ as a formal power series in $x^{-1}$ whose coefficients are $2 m \times 2 m$ matrices with entries from the algebra $\mathrm{U}(\mathfrak{g})$. Let $(x-\theta F)_{a b}^{-1}$ be the $a, b$ entry of the inverse matrix. It equals

$$
\delta_{a b} x^{-1}+\theta F_{a b} x^{-2}+\sum_{s=1}^{\infty} \sum_{\left|c_{1}\right|, \ldots,\left|c_{s}\right|=1}^{m} \theta^{s+1} F_{a c_{1}} F_{c_{1} c_{2}} \cdots F_{c_{s-1} c_{s}} F_{c_{s} b} x^{-s-2} .
$$

Like the indices $a$ and $b$, the indices $c_{1}, \ldots, c_{s}$ run through $-m, \ldots,-1,1, \ldots, m$. In another guise, the next result appeared first in [O2]. For connections to our present setting, see [KN3, Section 6] or [KN4, Section 6] when $\theta=1$ or $\theta=-1$, respectively.

To state the result in a way similar to Proposition 4.1 for $a<0$, put $p_{a i}=x_{-a, i}$ and $q_{a i}=\partial_{-a, i}$. For $a>0$, put $p_{a i}=-\theta \theta_{i} \partial_{a \tilde{\imath}}$ and $q_{a i}=\theta_{i} x_{a \tilde{\imath}}$. Then our definition of the homomorphism $\zeta: \mathrm{U}(\mathfrak{g}) \rightarrow \mathrm{H}(U)$ for $\mathfrak{g}=\mathfrak{s p}_{2 m}, \mathfrak{s o}_{2 m}$ can be written as

$$
\zeta\left(F_{a b}\right)=\theta \delta_{a b} n / 2-\sum_{i=1}^{n} \theta q_{a i} p_{b i} \quad \text { for } \quad a, b=-m, \ldots,-1,1, \ldots, m .
$$

Let $U(\mathfrak{g})^{G}$ be the subalgebra of $G$-invariant elements in $U(\mathfrak{g})$. It coincides with the centre of $\mathrm{U}(\mathfrak{g})$ if $\mathfrak{g}=\mathfrak{s p}_{2 m}$, but is strictly contained in the centre if $\mathfrak{g}=\mathfrak{s o}_{2 m}$.

Proposition 4.2. (i) One can define a homomorphism $\mathrm{X}\left(\mathfrak{g}^{\prime}\right) \rightarrow \mathrm{U}(\mathfrak{g}) \otimes \mathrm{H}(U)$ by

$$
S_{i j}(x) \mapsto \delta_{i j}+\sum_{|a|,|b|=1}^{m}\left(x \pm \frac{1}{2}+\theta m-\theta F\right)_{a b}^{-1} \otimes p_{a i} q_{b j}
$$

(ii) The subalgebra of $\mathrm{G}$-invariant elements in $\mathrm{A}=\mathrm{U}(\mathfrak{g}) \otimes \mathrm{H}(U)$ coincides with the subalgebra, generated by $\mathrm{U}(\mathfrak{g})^{\mathrm{G}} \otimes 1$ and by the image of the homomorphism (4.33).

Proof. Part (i) was proved in [KN3, Section 6] and [KN4, Section 6] for $\theta=1$ and $\theta=-1$, respectively. The claim that the image of the homomorphism (4.33) 
belongs to the subalgebra $\mathrm{A}^{\mathrm{G}} \subset \mathrm{A}$ was also proved therein. It remains to prove that the elements

$$
\sum_{|a|=1}^{m} 1 \otimes p_{a i} q_{a j}
$$

and

$$
\sum_{|a|,|b|,\left|c_{1}\right|, \ldots,\left|c_{s}\right|=1}^{m} F_{a c_{1}} F_{c_{1} c_{2}} \cdots F_{c_{s-1} c_{s}} E_{c_{s} b} \otimes p_{a i} q_{b j},
$$

taken together with the elements of $U(\mathfrak{g})^{\mathrm{G}} \otimes 1$, generate the whole subalgebra $\mathrm{A}^{\mathrm{G}} \subset \mathrm{A}$. Here $s=0,1,2, \ldots$ and $i, j=1, \ldots, n$. For $s=0$ the first tensor factor of the summand in (4.26) should be understood as $F_{a b}$.

Consider the standard $\mathbb{N}$-filtration on the algebra $U(\mathfrak{g})$, where any element of $\mathfrak{g}$ has degree 1 . The adjoint action of the group $\mathrm{G}$ on $\mathrm{U}(\mathfrak{g})$ preserves this filtration, and the corresponding graded algebra is identified with the symmetric algebra $\mathrm{S}(\mathfrak{g})$. The algebra $\mathrm{H}(U)$ is generated by its subspace $W=U \oplus U^{*}$, which is identified with the tensor product $\mathbb{C}^{2 m} \otimes \mathbb{C}^{n}$. By presenting $W$ as a direct sum of $n$ copies of $\mathbb{C}^{2 m}$, we get an $\mathbb{N}^{\times n}$-filtration on the algebra $\mathrm{H}(U)$, such that the $i$ th direct summand $\mathbb{C}^{2 m}$ of $W$ has degree 1 in the $i$ th factor $\mathbb{N}$ of $\mathbb{N} \times n$. This filtration is preserved by the action of the group $\mathrm{G}$ on $\mathrm{H}(U)$. The corresponding graded algebra is identified with $\mathrm{P}\left(\mathbb{C}^{2 m}\right)^{\otimes n}$. Here $\mathrm{P}\left(\mathbb{C}^{2 m}\right)$ denotes the symmetric algebra of $\mathbb{C}^{2 m}$ if $\theta=1$, or the exterior algebra of $\mathbb{C}^{2 m}$ if $\theta=-1$. It suffices to prove that the elements of the algebra $\mathrm{S}(\mathfrak{g}) \otimes \mathrm{P}\left(\mathbb{C}^{2 m}\right)^{\otimes n}$ corresponding to (4.34) and (4.35), taken together with the elements of the subalgebra

$$
\mathrm{S}(\mathfrak{g})^{\mathrm{G}} \otimes 1 \subset \mathrm{S}(\mathfrak{g}) \otimes \mathrm{P}\left(\mathbb{C}^{2 m}\right)^{\otimes n},
$$

generate the whole subalgebra of G-invariants. We will prove this by using the classical invariant theory for the group G; cf. [MO, Subsection 4.9].

Now $e_{a}$ with $a=-m, \ldots,-1,1, \ldots, m$ denotes a basis vector of $\mathbb{C}^{2 m}$. Consider the bilinear form on $\mathbb{C}^{2 m}$ preserved by the action of the group $\mathrm{G}$. Denote by $e_{a}^{\prime}$ the vector $\operatorname{sign} a \cdot e_{-a}$ or $e_{-a}$ when $\mathrm{G}$ is $\mathrm{Sp}_{2 m}$ or $\mathrm{O}_{2 m}$, respectively. The value of the form on a pair $\left(e_{a}, e_{b}^{\prime}\right)$ equals $\delta_{a b}$ for any indices $a$ and $b$. The odd tensor powers of $\mathbb{C}^{2 m}$ do not contain any nonzero $\mathrm{G}$-invariant vectors. The $\mathrm{G}$-invariant vectors in $\left(\mathbb{C}^{2 m}\right)^{\otimes 2 t}$ are all linear combinations of

$$
\sum_{\left|c_{1}\right|, \ldots,\left|c_{t}\right|=1}^{m} e_{c_{1}} \otimes e_{c_{1}}^{\prime} \otimes \cdots \otimes e_{c_{p}} \otimes e_{c_{t}}^{\prime}
$$

and of the sums, obtained from (4.37) by any simultaneous permutation of the $2 t$ tensor factors of every summand. For instance, see [H1, Theorem 1B].

First let us consider the G-invariants in the subspace

$$
1 \otimes \mathrm{P}\left(\mathbb{C}^{2 m}\right)^{\otimes n} \subset \mathrm{S}(\mathfrak{g}) \otimes \mathrm{P}\left(\mathbb{C}^{2 m}\right)^{\otimes n} .
$$

Regard the subspace of $\mathrm{P}\left(\mathbb{C}^{2 m}\right)^{\otimes n}$ of degree $\left(s_{1}, \ldots, s_{n}\right) \in \mathbb{N}^{\times n}$ as a subspace in the tensor product

$$
\left(\mathbb{C}^{2 m}\right)^{\otimes s_{1}} \otimes \cdots \otimes\left(\mathbb{C}^{2 m}\right)^{\otimes s_{n}}
$$

Our subspace is obtained by applying to each of the $n$ groups of the tensor factors $\mathbb{C}^{2 m}$ of (4.39) the symmetrization or anti-symmetrization for $\theta=1$ or $\theta=-1$, respectively. By using the explicit description of $\mathrm{G}$-invariants in (4.39) and arguing 
as in the proof of Proposition 4.1 one shows that the G-invariants in the subspace (4.38) are generated by elements of this subspace corresponding to (4.34) with $i, j=1, \ldots, n$. For example, if $n=2 t$, then (4.37) may be regarded as an element of the graded algebra $\mathrm{P}\left(\mathbb{C}^{2 m}\right)^{\otimes n}$ of degree $\left(s_{1}, \ldots, s_{n}\right)=(1, \ldots, 1)$. Then (4.37) corresponds to

$$
\sum_{\left|c_{1}\right|, \ldots,\left|c_{t}\right|=1}^{m} p_{c_{1} 1} q_{c_{1} 1} \cdots p_{c_{t} t} q_{c_{t} t} \in \mathrm{H}(U) .
$$

Now consider the subspace (4.36). Generators of the subalgebra $\mathrm{S}(\mathfrak{g})^{\mathrm{G}} \subset \mathrm{S}(\mathfrak{g})$ are well known. We will reproduce a set of generators here because they will be used in the subsequent argument. If $\mathrm{G}=\mathrm{Sp}_{2 m}$, then $\mathfrak{g}$ can be identified with the symmetric square of the G-module $\mathbb{C}^{2 m}$, so that $F_{a b} \in \mathfrak{g}$ is identified with the element

$$
\left(e_{a} \otimes e_{b}^{\prime}+e_{b}^{\prime} \otimes e_{a}\right) / 2
$$

of the symmetric square. If $\mathrm{G}=\mathrm{O}_{2 m}$, then $\mathfrak{g}$ can be identified with the exterior square of the G-module $\mathbb{C}^{2 m}$, so that $F_{a b} \in \mathfrak{g}$ is identified with the element

$$
\left(e_{a} \otimes e_{b}^{\prime}-e_{b}^{\prime} \otimes e_{a}\right) / 2
$$

of the exterior square. Now for $\mathrm{G}=\mathrm{Sp}_{2 m}, \mathrm{O}_{2 m}$, the subspace in $\mathrm{S}(\mathfrak{g})$ of degree $t$ becomes a subspace in the space of tensors $\left(\mathbb{C}^{2 m}\right)^{\otimes 2 t}$, and we may use the explicit description of $\mathrm{G}$-invariants in the latter space. This description implies that the elements of the graded algebra $\mathrm{S}(\mathfrak{g})$ corresponding to the elements

$$
\sum_{\left|c_{1}\right|, \ldots,\left|c_{s}\right|=1}^{m} F_{c_{1} c_{2}} F_{c_{2} c_{3}} \cdots F_{c_{s-1} c_{s}} F_{c_{s} c_{1}} \in \mathrm{U}(\mathfrak{g})^{\mathrm{G}}
$$

with $s=1,2, \ldots$ generate the subalgebra $\mathrm{S}(\mathfrak{g})^{\mathrm{G}} \subset \mathrm{S}(\mathfrak{g})$. A similar argument was given in the proof of Proposition 4.1; here we omit the details.

Now take the subspace in $\mathrm{S}(\mathfrak{g}) \otimes \mathrm{P}\left(\mathbb{C}^{2 m}\right)^{\otimes n}$ of degree $t \geqslant 1$ in the first tensor factor and of any nonzero degree $\left(s_{1}, \ldots, s_{n}\right)$ in the remaining $n$ tensor factors. Regard it as a subspace in

$$
\left(\mathbb{C}^{2 m}\right)^{\otimes 2 t} \otimes\left(\mathbb{C}^{2 m}\right)^{\otimes s_{1}} \otimes \cdots \otimes\left(\mathbb{C}^{2 m}\right)^{\otimes s_{n}}
$$

by using the embedding of $\mathfrak{g}$ into the tensor square of $\mathbb{C}^{2 m}$. Our subspace is obtained by applying to each of the first $t$ pairs of tensor factors $\mathbb{C}^{2 m}$ of 4.40) the symmetrization or anti-symmetrization for $\mathfrak{g}=\mathfrak{s p}_{2 m}$ or $\mathfrak{g}=\mathfrak{s o}_{2 m}$, respectively, by symmetrizing these $t$ pairs of tensor factors $\mathbb{C}^{2 m}$, and by applying to each of the $n$ groups of the remaining tensor factors $\mathbb{C}^{2 m}$ of (4.40) the symmetrization or anti-symmetrization for $\theta=1$ or $\theta=-1$, respectively. By using the explicit description of $\mathrm{G}$-invariants in (4.40) and arguing as we did in the case $\mathrm{G}=\mathrm{GL}_{m}$ of Proposition 4.1, we now complete the proof.

4.4. Tensor products. Recall a general definition. Let $\mathrm{F}$ be any algebraic group over $\mathbb{C}$ with a Lie algebra $\mathfrak{f}$. Let $\mathrm{E}$ be any associative algebra where the group $\mathrm{F}$ acts by automorphisms. Consider the crossed product algebra $\mathrm{F} \ltimes \mathrm{E}$. It is generated by the elements of $\mathrm{E}$ and $\mathrm{F}$ subject to the relations in $\mathrm{E}$ and $\mathrm{F}$ together with

$$
v Z v^{-1}=v(Z) \text { for } Z \in \mathrm{E} \text { and } v \in \mathrm{F} \text {. }
$$

Suppose there is also a homomorphism $\mathrm{U}(\mathfrak{f}) \rightarrow \mathrm{E}$. A module $K$ over the algebra $\mathrm{E}$ and over the group $\mathrm{F}$ will be called an $(\mathrm{E}, \mathrm{F})$-module if the joint actions of $\mathrm{E}$ 
and $\mathrm{F}$ on $K$ make it a module over the algebra $\mathrm{F} \ltimes \mathrm{E}$, and if the action of $\mathfrak{f}$ on $K$ corresponding to that of $\mathrm{F}$ coincides with the action of $\mathfrak{f}$ obtained by pulling the action of $\mathrm{E}$ on $K$ back through the homomorphism $\mathrm{U}(\mathfrak{f}) \rightarrow \mathrm{E}$. We suppose that $\mathrm{F}$ acts on $K$ locally finitely.

Take any reductive dual pair $\left(\mathrm{G}, \mathrm{G}^{\prime}\right)$ from Subsection 4.1. We have $U=\mathbb{C}^{m} \otimes \mathbb{C}^{n}$. The Lie algebra $\mathfrak{g}$ acts on the vector space $\mathrm{P}(U)$ via the homomorphism $\zeta: \mathrm{U}(\mathfrak{g}) \rightarrow$ $\mathrm{H}(U)$. The action of the algebra $\mathrm{H}(U)$ on $\mathrm{P}(U)$ is determined by (2.15). The group $\mathrm{G}^{\prime}$ acts on $\mathrm{H}(U)$ via linear transformations of the vector space $\mathbb{C}^{n}$. This action of $\mathrm{G}^{\prime}$ preserves the subspaces $U$ and $U^{*}$ of $\mathrm{H}(U)$. It also leaves invariant any element in the image of the homomorphism $\zeta$. Hence the actions of $\mathfrak{g}$ and $\mathrm{G}^{\prime}$ on $\mathrm{P}(U)$ commute with each other.

Now for any pair of weights $\lambda, \mu \in \mathfrak{t}^{*}$ consider the weight subspace

$$
\left(M_{\mu} \otimes \mathrm{P}(U)\right)_{\mathfrak{n}}^{\lambda} \subset\left(M_{\mu} \otimes \mathrm{P}(U)\right)_{\mathfrak{n}}
$$

of the space of $\mathfrak{n}$-coinvariants of tensor product of $\mathfrak{g}$-modules $M_{\mu} \otimes \mathrm{P}(U)$. We extend the action of the group $\mathrm{G}^{\prime}$ from $\mathrm{P}(U)$ to the latter tensor product, so that $\mathrm{G}^{\prime}$ acts on the tensor factor $M_{\mu}$ trivially. Then $\mathrm{G}^{\prime}$ also acts on the pair of vector spaces (4.41).

A bijective linear map

$$
\mathrm{P}(U) \rightarrow\left(M_{\mu} \otimes \mathrm{P}(U)\right)_{\mathfrak{n}}
$$

can be defined by mapping any $u \in \mathrm{P}(U)$ to the coset of $1_{\mu} \otimes u$ in $\left(M_{\mu} \otimes \mathrm{P}(U)\right)_{\mathfrak{n}}$. The algebra A acts on the vector space $M_{\mu} \otimes \mathrm{P}(U)$ by definition. By restricting the latter action to the subalgebra $\mathrm{U}(\mathfrak{g}) \subset \mathrm{A}$, we get the diagonal action of $\mathfrak{g}$ on $M_{\mu} \otimes \mathrm{P}(U)$, as above. When the A-module $M_{\mu} \otimes \mathrm{P}(U)$ is identified with $\mathrm{A}_{\mu}$ as in Subsection 2.3, then the pair of vector spaces (4.41) gets identified with the pair $\mathrm{M}_{\mu}^{\lambda} \subset \mathrm{M}_{\mu}$.

The group $\mathrm{G}^{\prime}$ acts by automorphisms of the algebra $\mathrm{A}=\mathrm{U}(\mathfrak{g}) \otimes \mathrm{H}(U)$ via its action on $\mathrm{H}(U)$. The action of $\mathrm{G}^{\prime}$ on $\mathrm{A}$ preserves each of the ideals $\mathrm{I}, \mathrm{J}$ and $\mathrm{J}_{\mu}^{\prime \prime}$. Hence $\mathrm{G}^{\prime}$ also acts on the pair $\mathrm{M}_{\mu}^{\lambda} \subset \mathrm{M}_{\mu}$. Its identification with (4.41) is that of pairs of $\mathrm{G}^{\prime}$-modules.

Note that the action of the Lie group $\mathrm{G}^{\prime}$ on the subspace $\mathrm{P}(U) \subset \mathrm{H}(U)$ gives a homomorphism of algebras $\mathrm{U}\left(\mathfrak{g}^{\prime}\right) \rightarrow \mathrm{H}(U)$. Any element of its image is $\mathrm{G}$-invariant. For $\mathrm{G}^{\prime}=\mathrm{GL}_{n}$, the image of the element $E_{i j} \in \mathfrak{g l}_{n}$ under this homomorphism equals (4.3). For $\mathrm{G}^{\prime}=\mathrm{O}_{n}$ or $\mathrm{G}^{\prime}=\mathrm{Sp}_{n}$ the image of the element $E_{i j}-\theta_{i} \theta_{j} E_{\tilde{\jmath} \tau} \in \mathfrak{g}^{\prime}$ equals (4.4). By identifying $\mathrm{H}(U)$ with the subalgebra $1 \otimes \mathrm{H}(U) \subset \mathrm{A}$, we get a homomorphism $\mathrm{U}\left(\mathfrak{g}^{\prime}\right) \rightarrow \mathrm{A}^{\mathrm{G}}$. Then (4.41) is a pair of $\left(\mathrm{A}^{\mathrm{G}}, \mathrm{G}^{\prime}\right)$-modules, and so is the pair $\mathrm{M}_{\mu}^{\lambda} \subset \mathrm{M}_{\mu}$.

More generally, for any element $\omega$ of the group $\mathfrak{R}=\mathfrak{S} \ltimes \mathfrak{T}$, consider the subspace

$$
\left(M_{\omega \circ \mu} \otimes \mathrm{P}(\widehat{\omega}(U))\right)_{\mathfrak{n}}^{\omega \circ \lambda} \subset\left(M_{\omega \circ \mu} \otimes \mathrm{P}(\widehat{\omega}(U))\right)_{\mathfrak{n}}
$$

of the space of $\mathfrak{n}$-coinvariants of the tensor product of $\mathfrak{g}$-modules $M_{\omega \circ \mu} \otimes \mathrm{P}(\widehat{\omega}(U))$. A bijective linear map

$$
\mathrm{P}(\widehat{\omega}(U)) \rightarrow\left(M_{\omega \circ \mu} \otimes \mathrm{P}(\widehat{\omega}(U))\right)_{\mathfrak{n}}
$$

can be defined by mapping any $w \in \mathrm{P}(\widehat{\omega}(U))$ to the coset of $1_{\omega \circ \mu} \otimes w$ in the space of coinvariants. Similarly to (2.15), the action of the algebra $\mathrm{H}(U)$ on $\mathrm{P}(\widehat{\omega}(U))$ is defined by

$$
X(u)=v \quad \text { if } \quad X u-v \in \mathrm{H}(U) \cdot \widehat{\omega}\left(U^{*}\right),
$$


where $u, v \in \mathrm{P}(\widehat{\omega}(U))$. Then the Lie algebra $\mathfrak{g}$ acts on the vector space $\mathrm{P}(\widehat{\omega}(U))$ via the homomorphism $\zeta: \mathrm{U}(\mathfrak{g}) \rightarrow \mathrm{H}(U)$. The pair of vector spaces (4.43) gets identified with the pair

$$
\mathrm{M}_{\omega \circ \mu, \widehat{\omega}\left(U^{*}\right)}^{\omega \circ \lambda} \subset \mathrm{M}_{\omega \circ \mu, \widehat{\omega}\left(U^{*}\right)} .
$$

The action of $\mathrm{G}^{\prime}$ on $\mathrm{H}(U)$ commutes with that of $\mathrm{G}$. In particular, the action of $\mathrm{G}^{\prime}$ preserves the subspaces $\widehat{\omega}(U)$ and $\widehat{\omega}\left(U^{*}\right)$ of $\mathrm{H}(U)$. Hence the group $\mathrm{G}^{\prime}$ also acts on the two pairs (4.43) and (4.45). Our identification of them is that of pairs of $\mathrm{G}^{\prime}$-modules. Note that both (4.43) and (4.45) are also $\left(\mathrm{A}^{\mathrm{G}}, \mathrm{G}^{\prime}\right)$-modules, as they were in the particular case above when $\omega$ was the identity element of $\Re$.

The subalgebra $\mathrm{A}^{\mathrm{G}}$ of $\mathrm{G}$-invariant elements of $\mathrm{A}$ acts on the weight subspace (4.43) by definition. First suppose that $\mathrm{G}=\mathrm{GL}_{m}$. As in the previous subsection, we will treat the cases of $\theta=1$ and $\theta=-1$ simultaneously. Via the homomorphism $\mathrm{Y}\left(\mathfrak{g l}_{n}\right) \rightarrow \mathrm{A}^{\mathrm{GL}_{m}}$ defined by (4.24), the subspace in (4.43) becomes a module over the Yangian $\mathrm{Y}\left(\mathfrak{g l}_{n}\right)$. We shall now describe this module explicitly, by using the comultiplication (4.10) and the homomorphism (4.8). For $\mathfrak{g}=\mathfrak{g l}_{m}$ the group $\mathfrak{T}$ is trivial, while any $\widehat{\omega}$ preserves $U$ and $U^{*}$. Hence it suffices to consider only the case when $\omega$ is the identity element.

Every weight $\mu$ of $\mathfrak{g l}_{m}$ is determined by the sequence $\mu_{1}, \ldots, \mu_{m}$ of its labels where $\mu_{a}=\mu\left(E_{a a}\right)$ for each $a=1, \ldots, m$. Note that here for the half-sum $\rho$ of the positive roots $\rho_{a}=m / 2-a+\frac{1}{2}$. For $\mathfrak{g}=\mathfrak{g l}_{m}$ we get $\kappa=0$ by the definitions (2.12), (4.1). Then $\nu=\lambda-\mu$ by (2.18). Take the sequence $\nu_{1}, \ldots, \nu_{m}$ of labels of $\nu$. If $\theta=1$, then suppose that each label $\nu_{a} \in\{0,1,2, \ldots\}$. If $\theta=-1$, then suppose that each $\nu_{a} \in\{0,1, \ldots, n\}$. Otherwise the weight subspace in (4.41) would contain zero only.

If $\theta=1$, then denote by $\Phi^{k}$ the $k$ th symmetric power of the defining $\mathfrak{g l}_{n}$-module $\mathbb{C}^{n}$. If $\theta=-1$, then denote by the same symbol $\Phi^{k}$ the $k$ th exterior power of the $\mathfrak{g l}_{n}$-module $\mathbb{C}^{n}$. The group $\mathrm{G}^{\prime}=\mathrm{GL}_{n}$ also acts on $\Phi^{k}$. Using the homomorphism (4.8), regard $\Phi^{k}$ as a module over the Yangian $\mathrm{Y}\left(\mathfrak{g l}_{n}\right)$. For $t \in \mathbb{C}$ denote by $\Phi_{t}^{k}$ the $\mathrm{Y}\left(\mathfrak{g l}_{n}\right)$-module obtained by pulling the $\mathrm{Y}\left(\mathfrak{g l}_{n}\right)$-module $\Phi^{k}$ back through the automorphism (4.6) where $z=\theta t$.

For $\theta=1$, denote by $\mathrm{P}\left(\mathbb{C}^{n}\right)$ the symmetric algebra of the vector space $\mathbb{C}^{n}$. For $\theta=-1$, denote by $\mathrm{P}\left(\mathbb{C}^{n}\right)$ the exterior algebra of $\mathbb{C}^{n}$. For any $\theta$ and $t$ the underlying vector space of the $\mathrm{Y}\left(\mathfrak{g l}_{n}\right)$-module $\Phi_{t}^{k}$ consists of all homogeneous elements of $\mathrm{P}\left(\mathbb{C}^{n}\right)$ of degree $k$. Using the standard basis $e_{1}, \ldots, e_{m}$ of $\mathbb{C}^{m}$, decompose the vector space $U=\mathbb{C}^{m} \otimes \mathbb{C}^{n}$ into a direct sum of $m$ copies of $\mathbb{C}^{n}$. Then the vector space $\mathrm{P}(U)$ gets identified with the tensor product of $m$ copies of $\mathrm{P}\left(\mathbb{C}^{n}\right)$. For the proof of the following proposition, see [KN1, Section 2] or [KN2, Section 2] when $\theta=1$ or $\theta=-1$, respectively.

Proposition 4.3. Under the above assumptions on the weight $\nu$, the subspace in (4.41) is equivalent as a $\mathrm{Y}\left(\mathfrak{g l}_{n}\right)$-module to the tensor product

$$
\Phi_{\mu_{1}+\rho_{1}+\frac{1}{2}}^{\nu_{1}} \otimes \cdots \otimes \Phi_{\mu_{m}+\rho_{m}+\frac{1}{2}}^{\nu_{m}} .
$$

An equivalence mapping from the latter $\mathrm{Y}\left(\mathfrak{g l}_{n}\right)$-module to the former is defined by regarding (4.46) as a subspace of $\mathrm{P}(U)$, and then applying the map (4.42) to that subspace.

The equivalence mapping here is also that of $\mathrm{GL}_{n}$-modules. This follows from the $\mathrm{GL}_{n}$-equivariance of the map (4.42). The group $\mathrm{GL}_{n}$ also acts by automorphisms of 
the Hopf algebra $\mathrm{Y}\left(\mathfrak{g l}_{n}\right)$. When an element $v \in \mathrm{GL}_{n}$ is regarded as an $n \times n$ matrix by using the standard basis $f_{1}, \ldots, f_{n}$ of $\mathbb{C}^{n}$, the corresponding automorphism of $\mathrm{Y}\left(\mathfrak{g l}_{n}\right)$ is defined by

$$
T(x) \mapsto v^{-1} T(x) v .
$$

The Olshanski homomorphism $\mathrm{Y}\left(\mathfrak{g l}_{n}\right) \rightarrow \mathrm{A}^{\mathrm{GL}_{m}}$ is $\mathrm{GL}_{n}$-equivariant. This can be verified directly by using the definition (4.24). Furthermore, we can consider the crossed product $\mathrm{GL}_{n} \ltimes \mathrm{Y}\left(\mathfrak{g l}_{n}\right)$. Moreover, we have an embedding $\mathrm{U}\left(\mathfrak{g l}_{n}\right) \rightarrow \mathrm{Y}\left(\mathfrak{g l}_{n}\right)$. Then both modules in Proposition 4.3 become $\left(\mathrm{Y}\left(\mathfrak{g l}_{n}\right), \mathrm{GL}_{n}\right)$-modules. For the latter module this can be verified directly by using the definition of $\Phi_{t}^{k}$. For the former this follows from $\mathrm{GL}_{n}$-equivariance of (4.24) by regarding $M_{\mu} \otimes \mathrm{P}(U)$ as a module over the algebra $\mathrm{GL}_{n} \ltimes \mathrm{A}$.

Now take $\omega=\sigma$ with arbitrary $\sigma \in \mathfrak{S}$. By replacing in Proposition 4.3 the weights $\lambda$ and $\mu$ by $\sigma \circ \lambda$ and $\sigma \circ \mu$, respectively, we get a description of the subspace in (4.43) with $\omega=\sigma$ as a module over the Yangian $\mathrm{Y}\left(\mathfrak{g l}_{n}\right)$. Then $\nu$ is replaced by $\sigma(\nu)$. Let us identify the Weyl group $\mathfrak{S}$ with the symmetric group $\mathfrak{S}_{m}$ so that any permutation of the dual basis vectors $\eta_{1}, \ldots, \eta_{m} \in \mathfrak{t}^{*}$ by an element $\sigma \in \mathfrak{S}$ corresponds to a permutation of the numbers $1, \ldots, m$. The latter permutation will be denoted by the same symbol $\sigma$. Put

$$
\breve{\mu}_{a}=\mu_{\sigma^{-1}(a)}, \quad \breve{\nu}_{a}=\nu_{\sigma^{-1}(a)}, \quad \breve{\rho}_{a}=\rho_{\sigma^{-1}(a)} .
$$

These are the ath labels of the weights $\sigma(\mu), \sigma(\nu), \sigma(\rho)$, respectively. Using this notation, the subspace in (4.43) with $\omega=\sigma$ is equivalent as a module over $\mathrm{Y}\left(\mathfrak{g l}_{n}\right)$ and $\mathrm{GL}_{n}$ to the tensor product

$$
\Phi_{\breve{\mu}_{1}+\breve{\rho}_{1}+\frac{1}{2}}^{\breve{\nu}_{1}} \otimes \cdots \otimes \Phi_{\breve{\mu}_{m}+\breve{\rho}_{m}+\frac{1}{2}}^{\breve{\nu}_{m}} .
$$

Now suppose that the weight $\lambda+\rho$ of $\mathfrak{g l}_{m}$ is dominant. By Corollary 3.6, the Zhelobenko operator $\check{\xi}_{\sigma}$ on $\overline{\mathrm{A}} / \overline{\mathrm{J}}$ then defines a linear map (3.15). This map commutes with the actions of the algebra $\mathrm{A}^{\mathrm{GL}_{m}}$ on the source and the target vector spaces of (3.15). Hence it is an intertwining operator of $\mathrm{Y}\left(\mathfrak{g l}_{n}\right)$-modules. By replacing the modules by their equivalents, we now get an intertwining operator

$$
\Phi_{\mu_{1}+\rho_{1}+\frac{1}{2}}^{\nu_{1}} \otimes \cdots \otimes \Phi_{\mu_{m}+\rho_{m}+\frac{1}{2}}^{\nu_{m}} \rightarrow \Phi_{\breve{\mu}_{1}+\breve{\rho}_{1}+\frac{1}{2}}^{\breve{\nu}_{1}} \otimes \cdots \otimes \Phi_{\breve{\mu}_{m}+\breve{\rho}_{m}+\frac{1}{2}}^{\breve{\nu}_{m}}
$$

of two tensor products of $\mathrm{Y}\left(\mathfrak{g l}_{n}\right)$-modules. Both tensor products are irreducible and equivalent to each other if (but not only if) the weight $\mu$ is generic, that is, if $\mu_{a}-\mu_{b} \notin \mathbb{Z}$ whenever $a \neq b$; see [NT2, Theorem 4.8] for a more general result. Hence for generic $\mu$, an intertwining operator between the two tensor products is unique up to a scalar factor. For our particular intertwining operator, this factor is determined by the next proposition. This proposition is valid for any weight $\mu$, not necessarily generic.

Choose $\varphi_{k} \in \Phi^{k}$ as follows. If $\theta=1$, then $\Phi^{k}$ is the $k$ th symmetric power of $\mathbb{C}^{n}$, and we put $\varphi_{k}=f_{1}^{k}$. If $\theta=-1$, then $\Phi^{k}$ is the $k$ th exterior power of $\mathbb{C}^{n}$, and we put $\varphi_{k}=f_{1} \wedge \cdots \wedge f_{k}$. The vector $\varphi_{k}$ is annihilated by the action of the elements $E_{i j} \in \mathfrak{g l}_{n}$ with $i<j$. Take any positive root $\alpha=\eta_{a}-\eta_{b}$ of $\mathfrak{g l}_{m}$ with $a<b$. For $\theta=1$, define

$$
z_{\alpha}=\prod_{s=1}^{\nu_{b}} \frac{\mu_{a}-\mu_{b}+\rho_{a}-\rho_{b}-s}{\lambda_{a}-\lambda_{b}+\rho_{a}-\rho_{b}+s} .
$$


Here the denominator corresponding to the running index $s$ equals $(\lambda+\rho)\left(H_{\alpha}\right)+s$. Hence the denominator does not vanish for dominant $\lambda+\rho$. For $\theta=-1$, define

$$
z_{\alpha}=(-1)^{\nu_{a} \nu_{b}}\left\{\begin{array}{ccc}
\frac{\lambda_{a}-\lambda_{b}+\rho_{a}-\rho_{b}}{\mu_{a}-\mu_{b}+\rho_{a}-\rho_{b}} & \text { if } & \nu_{a}<\nu_{b} ; \\
1 & \text { if } & \nu_{a} \geqslant \nu_{b} .
\end{array}\right.
$$

Here $\mu_{a}=\lambda_{a}-\nu_{a}$ for any index $a$. Therefore in the first of the last two cases, the denominator equals $(\lambda+\rho)\left(H_{\alpha}\right)-\nu_{a}+\nu_{b}$. Hence it does not vanish for dominant $\lambda+\rho$, under the condition $\nu_{a}<\nu_{b}$ when this denominator occurs.

Proposition 4.4. Let $\lambda+\rho$ be dominant. The operator (4.48) determined by $\check{\xi}_{\sigma}$ maps the vector $\varphi_{\nu_{1}} \otimes \cdots \otimes \varphi_{\nu_{m}}$ of (4.46) to the vector $\varphi_{\breve{\nu}_{1}} \otimes \cdots \otimes \varphi_{\breve{\nu}_{m}}$ of (4.47) multiplied by all those $z_{\alpha}$ where $\alpha \in \Delta^{+}$but $\sigma(\alpha) \notin \Delta^{+}$.

Proof. For a generic weight $\mu$, this proposition has been proved in [KN1, Section 3] and [KN2, Section 3] when $\theta=1$ or $\theta=-1$, respectively. But when $\nu$ is fixed, our operator (4.48) depends on $\mu \in \mathfrak{t}^{*}$ continuously; see the proof of Corollary 3.6.

For any $\mu$ and dominant $\lambda+\rho$, Corollary 3.9 and Proposition 4.1 give that for $\sigma=\sigma_{0}$ the quotient by the kernel, or equivalently, the image of our operator

$$
\Phi_{\mu_{1}+\rho_{1}+\frac{1}{2}}^{\nu_{1}} \otimes \cdots \otimes \Phi_{\mu_{m}+\rho_{m}+\frac{1}{2}}^{\nu_{m}} \rightarrow \Phi_{\mu_{m}+\rho_{m}+\frac{1}{2}}^{\nu_{m}} \otimes \cdots \otimes \Phi_{\mu_{1}+\rho_{1}+\frac{1}{2}}^{\nu_{1}}
$$

is an irreducible $\mathrm{Y}\left(\mathfrak{g l}_{n}\right)$-module. Here we also use the observation that any element of the subalgebra $\mathrm{U}\left(\mathfrak{g l}_{m}\right)^{\mathrm{GL}_{m}} \otimes 1 \subset \mathrm{A}^{\mathrm{GL}_{m}}$ acts on the subspace (4.41) by scalar multiplication. We will show in Section 5 that up to equivalence and similarity, every irreducible finite-dimensional $\mathrm{Y}\left(\mathfrak{g l}_{n}\right)$-module arises as the image of (4.49) for $\theta=-1$ and some $\lambda, \mu$.

Now let $\left(G, G^{\prime}\right)$ be any of the reductive dual pairs from (0.1) with $G=\operatorname{Sp}_{2 m}$ or $\mathrm{G}=\mathrm{O}_{2 m}$. We will continue treating the cases of $\theta=1$ and $\theta=-1$ simultaneously. Via the homomorphism $\mathrm{X}\left(\mathfrak{g}^{\prime}\right) \rightarrow \mathrm{A}^{\mathrm{G}}$ defined by (4.33), the subspace in (4.43) becomes a module over the extended twisted Yangian $\mathrm{X}\left(\mathfrak{g}^{\prime}\right)$. We shall now describe this module explicitly. For $\mathfrak{g} \neq \mathfrak{g l}_{m}$ we do not have $\widehat{\omega}(U)=U$ in general. We will give an analogue of Proposition 4.3 for arbitrary $\omega \in \mathfrak{R}$. For the proof of this analogue, see [KN3, Section 5] and [KN4, Section 5] in the cases $\theta=1$ and $\theta=-1$, respectively. We will keep using the $\mathrm{Y}\left(\mathfrak{g l}_{n}\right)$-modules $\Phi_{t}^{k}$ for integers $k \geqslant 0$. Further, denote by $\Phi_{t}^{-k}$ the $\mathrm{Y}\left(\mathfrak{g l}_{n}\right)$-module obtained by pulling the $\mathrm{Y}\left(\mathfrak{g l}_{n}\right)$-module $\Phi_{t}^{k}$ back through the automorphism (4.12).

We regard $\mathfrak{g}^{\prime}$ as a Lie subalgebra of $\mathfrak{g l}_{n}$. Any $\mathrm{Y}\left(\mathfrak{g l}_{n}\right)$-module can also be regarded as an $\mathrm{X}\left(\mathfrak{g}^{\prime}\right)$-module, first by restricting from $\mathrm{Y}\left(\mathfrak{g l}_{n}\right)$ to the subalgebra $\mathrm{Y}\left(\mathfrak{g}^{\prime}\right)$, and then by pulling back through the homomorphism $\mathrm{X}\left(\mathfrak{g}^{\prime}\right) \rightarrow \mathrm{Y}\left(\mathfrak{g}^{\prime}\right)$ defined by (4.17). The resulting $\mathrm{X}\left(\mathfrak{g}^{\prime}\right)$-module can also be defined as the tensor product of the initial $\mathrm{Y}\left(\mathfrak{g l}_{n}\right)$-module by the one-dimensional trivial $\mathrm{X}\left(\mathfrak{g}^{\prime}\right)$-module, which is defined by the assignment $S_{i j}(x) \mapsto \delta_{i j}$. Here we use the coaction of $\mathrm{Y}\left(\mathfrak{g l}_{n}\right)$ on $\mathrm{X}\left(\mathfrak{g}^{\prime}\right)$ defined by (4.23). Either way, any tensor product of $\mathrm{Y}\left(\mathrm{gl}_{n}\right)$-modules of the form $\Phi_{t}^{k}$ or $\Phi_{t}^{-k}$ will be regarded as an $\mathrm{X}\left(\mathfrak{g}^{\prime}\right)$-module. The group $\mathrm{G}^{\prime}$ acts on $\Phi_{t}^{k}$ and on $\Phi_{t}^{-k}$ by restricting the natural action of $\mathrm{GL}_{n}$ on $\Phi^{k}$.

For any weight $\mu$ of $\mathfrak{g}=\mathfrak{s p}_{2 m}$ or $\mathfrak{g}=\mathfrak{s o}_{2 m}$, define the sequence $\mu_{1}, \ldots, \mu_{m}$ of its labels by setting $\mu_{a}=\mu\left(F_{a a}\right)$ for $a=1, \ldots, m$. Note that unlike in the case of $\mathfrak{g}=\mathfrak{g l}_{m}$, here

$$
\mu=-\mu_{m} \eta_{1}-\cdots-\mu_{1} \eta_{m}
$$


For the half-sum of positive roots we get $\rho_{a}=-a$ if $\mathfrak{g}=\mathfrak{s p}_{2 m}$, or $\rho_{a}=1-a$ if $\mathfrak{g}=\mathfrak{s o}_{2 m}$. By the definitions (2.12), (4.2) here $\kappa_{a}=\theta n / 2$. Consider the sequence $\nu_{1}, \ldots, \nu_{m}$ of the labels the weight (2.18). As for $\mathfrak{g}=\mathfrak{g l}_{m}$, suppose that each label $\nu_{a} \in\{0,1,2, \ldots\}$ if $\theta=1$. If $\theta=-1$, then suppose that each $\nu_{a} \in\{0,1, \ldots, n\}$. Otherwise the weight subspace in (4.43) would contain zero only.

The Weyl group of $\mathfrak{s p}_{2 m}$ is isomorphic to the semidirect product $\mathfrak{S}_{m} \ltimes \mathbb{Z}_{2}^{m}$; the group $\mathfrak{T}$ is trivial in this case. The Weyl group of $\mathfrak{s o}_{2 m}$ is isomorphic to a subgroup of $\mathfrak{S}_{m} \ltimes \mathbb{Z}_{2}^{m}$ of index two, and $\mathfrak{T}$ is isomorphic to $\mathbb{Z}_{2}$. The extended Weyl group $\mathfrak{R}=$ $\mathfrak{S} \ltimes \mathfrak{T}$ of $\mathfrak{s o}_{2 m}$ is isomorphic to $\mathfrak{S}_{m} \ltimes \mathbb{Z}_{2}^{m}$. Fix the isomorphisms as follows. Regard $\mathfrak{S}_{m} \ltimes \mathbb{Z}_{2}^{m}$ as the group of permutations of the indices $-m, \ldots,-1,1, \ldots, m$ such that if $a \mapsto b$ under a permutation, then $-a \mapsto-b$ under the same permutation. The image of $\omega \in \mathfrak{R}$ in $\mathfrak{S}_{m} \ltimes \mathbb{Z}_{2}^{m}$ will be denoted by $\bar{\omega}$. Then for any $c=1, \ldots, m-1$ the permutation $\bar{\sigma}_{c}$ only exchanges $c-m-1$ with $c-m$, and $m-c$ with $m-c+1$. For $\mathfrak{g}=\mathfrak{s p}_{2 m}$, the permutation $\bar{\sigma}_{m}$ exchanges only -1 and 1 . For $\mathfrak{g}=\mathfrak{s o}_{2 m}$ the transposition of -1 and 1 will be denoted by $\bar{\tau}_{m}$; then $\bar{\sigma}_{m}=\bar{\tau}_{m} \bar{\sigma}_{m-1} \bar{\tau}_{m}$. The transposition $\bar{\tau}_{m}$ is the image in $\mathfrak{S}_{m} \ltimes \mathbb{Z}_{2}^{m}$ of the generator $\tau_{m}$ of $\mathfrak{T}$ for $\mathfrak{g}=\mathfrak{s o}_{2 m}$.

Now put

$$
\breve{\mu}_{a}=\mu_{\left|\bar{\omega}^{-1}(a)\right|}, \quad \breve{\nu}_{a}=\nu_{\left|\bar{\omega}^{-1}(a)\right|}, \quad \breve{\rho}_{a}=\rho_{\left|\bar{\omega}^{-1}(a)\right|}, \quad \text { and } \quad \delta_{a}=\operatorname{sign} \bar{\omega}^{-1}(a)
$$

for any $a=1, \ldots, m$. Then the $a$ th label of the weight $\omega(\mu)$ is equal to $\delta_{a} \breve{\mu}_{a}$.

For any $a=1, \ldots, m$, let $\widehat{\omega}_{a}$ be the element of the group $G$ such that $\widehat{\omega}_{a}\left(e_{-a}\right)=$ $e_{a}$, and $\widehat{\omega}_{a}\left(e_{a}\right)=-e_{-a}$ or $\widehat{\omega}_{a}\left(e_{a}\right)=e_{-a}$ depending on whether $\mathrm{G}=\operatorname{Sp}_{2 m}$ or $\mathrm{G}=\mathrm{O}_{2 m}$. By definition, all other basis vectors of $\mathbb{C}^{2 m}$ are invariant under the action of $\widehat{\omega}_{a}$. We have $\widehat{\omega}_{a} \in$ Norm $T$. The image of $\widehat{\omega}_{a}$ in the group $\mathfrak{R}$ will be denoted by $\omega_{a}$. In particular, $\omega_{1}=\sigma_{m}$ in the case $\mathrm{G}=\mathrm{Sp}_{2 m}$, but $\omega_{1}=\tau_{m}$ in the case $\mathrm{G}=\mathrm{O}_{2 m}$. The action of $\widehat{\omega}_{a} \in \mathrm{G}$ on the vector space $W=\mathbb{C}^{2 m} \otimes \mathbb{C}^{n}$ determines an automorphism of the algebra $\mathrm{H}(U)$ such that for all $i=1, \ldots, n$

$$
x_{a i} \mapsto-\theta \theta_{i} \partial_{a \tilde{\imath}} \quad \text { and } \quad \partial_{a i} \mapsto \theta_{i} x_{a \tilde{\imath}},
$$

while the elements $x_{b i}, \partial_{b i} \in \mathrm{H}(U)$ with the indices $b \neq a$ are invariant under this automorphism. This automorphism of $\mathrm{H}(U)$ will denoted by the same symbol $\widehat{\omega}_{a}$.

Proposition 4.5. Under the above assumptions on the weight $\nu$, the subspace in (4.43) is equivalent as module over $\mathrm{X}\left(\mathfrak{g}^{\prime}\right)$ to the tensor product

$$
\Phi_{\breve{\mu}_{1}+\breve{\rho}_{1}+\frac{1}{2}}^{\delta_{\breve{\nu}_{1}}} \otimes \cdots \otimes \Phi_{\breve{\mu}_{m}+\breve{\rho}_{m}+\frac{1}{2}}^{\delta_{m} \breve{L}_{m}}
$$

pulled back through the automorphism (4.15) of $\mathrm{X}\left(\mathfrak{g}^{\prime}\right)$, where

$$
f(x)=\prod_{a=1}^{m} \frac{\theta x-\mu_{a}-\rho_{a}-\frac{1}{2}}{\theta x-\mu_{a}-\rho_{a}+\frac{1}{2}} .
$$

An equivalence mapping from the latter $\mathrm{X}\left(\mathfrak{g}^{\prime}\right)$-module to the former is defined by regarding (4.51) as a subspace of $\mathrm{P}(U) \subset \mathrm{H}(U)$, then applying to this subspace all automorphisms $\widehat{\omega}_{a}^{-1}$ with $\delta_{a}=-1$, and then applying the map (4.44) to the resulting subspace of $\mathrm{H}(U)$.

The equivalence mapping here is also that of $\mathrm{G}^{\prime}$-modules. This follows from the $\mathrm{G}^{\prime}$-equivariance of the map (4.44). The group $\mathrm{G}^{\prime}$ also acts by automorphisms of the right coideal subalgebra $\mathrm{Y}\left(\mathfrak{g}^{\prime}\right) \subset \mathrm{Y}\left(\mathfrak{g l}_{n}\right)$, and by automorphisms of the right $\mathrm{Y}\left(\mathfrak{g l}_{n}\right)$-comodule algebra $\mathrm{X}\left(\mathfrak{g}^{\prime}\right)$. When an element $v \in \mathrm{G}^{\prime}$ is regarded as 
an $n \times n$ matrix by using the standard basis $f_{1}, \ldots, f_{n}$ of $\mathbb{C}^{n}$, the corresponding automorphism of $\mathrm{X}\left(\mathfrak{g}^{\prime}\right)$ is defined by

$$
S(x) \mapsto v^{-1} S(x) v .
$$

It factors to an automorphism of the quotient $Y\left(\mathfrak{g}^{\prime}\right)$ of the algebra $\mathrm{X}\left(\mathfrak{g}^{\prime}\right)$. The Olshanski homomorphism $\mathrm{X}\left(\mathfrak{g}^{\prime}\right) \rightarrow \mathrm{A}^{\mathrm{G}}$ is $\mathrm{G}^{\prime}$-equivariant. This can be verified directly by using the definition (4.33). We can consider the crossed product algebra $\mathrm{G}^{\prime} \ltimes \mathrm{Y}\left(\mathfrak{g}^{\prime}\right)$. We also have an embedding $\mathrm{U}\left(\mathfrak{g}^{\prime}\right) \rightarrow \mathrm{Y}\left(\mathfrak{g}^{\prime}\right)$, defined by mapping each element $E_{i j}-\theta_{i} \theta_{j} E_{\tilde{\jmath} \tilde{\imath}} \in \mathfrak{g}^{\prime}$ to the coefficient at $x^{-1}$ of the series (4.13). Then (4.51) becomes a $\left(\mathrm{Y}\left(\mathfrak{g}^{\prime}\right), \mathrm{G}^{\prime}\right)$-module. This statement can be verified directly by using the definitions of $\Phi_{t}^{k}$ and $\Phi_{t}^{-k}$.

Note that the series $f(x)$ in Proposition 4.5 does not depend on the choice of $\omega \in \mathfrak{R}$. By using this proposition in the basic case when $\omega$ is the identity element, the subspace in (4.41) is equivalent as an $\mathrm{X}\left(\mathfrak{g}^{\prime}\right)$-module to the tensor product of the form (4.46) pulled back through the automorphism (4.15) of $\mathrm{X}\left(\mathfrak{g}^{\prime}\right)$ where the series $f(x)$ is as above. But here the labels $\mu_{a}, \nu_{a}, \rho_{a}$ for $a=1, \ldots, m$ correspond to the Lie algebra $\mathfrak{g}=\mathfrak{s p}_{2 m}$ or $\mathfrak{g}=\mathfrak{s o}_{2 m}$, not to $\mathfrak{g}=\mathfrak{g l}_{m}$. In particular, here the labels $\rho_{a}$ of the half-sum $\rho$ of the positive roots are different from those for $\mathfrak{g}=\mathfrak{g l}_{m}$. Our choice (4.50) of the labels of $\mu$ for $\mathfrak{g}=\mathfrak{s p}_{2 m}$ or $\mathfrak{g}=\mathfrak{s o}_{2 m}$ has been made so that here the description of the subspace (4.41) becomes similar to that in the case $\mathfrak{g}=\mathfrak{g l}_{m}$. For the same purpose, in the definition (4.24) we employed the matrix $(x+\theta m / 2+\theta E)^{-1}$ rather than the matrix $(x+\theta E)^{-1}$.

Now we suppose that the weight $\lambda+\rho$ of $\mathfrak{g}=\mathfrak{s p}_{2 m}$ or $\mathfrak{g}=\mathfrak{s o}_{2 m}$ is dominant. Then by Corollary 3.6, the Zhelobenko operator $\check{\xi}_{\sigma}$ on $\overline{\mathrm{A}} / \overline{\mathrm{J}}$ determines a linear map (3.15). This map commutes with the actions of the algebra $\mathrm{A}^{\mathrm{G}}$ on the source and target vector spaces of (3.15). Further, for $\mathfrak{g}=\mathfrak{s o}_{2 m}$ and $\omega=\tau_{m} \sigma$ the representative $\widehat{\tau}_{m} \in \mathrm{G}$ of $\tau \in \mathfrak{T}$ yields a linear map

$$
\mathrm{M}_{\sigma \circ \mu, \widehat{\sigma}\left(U^{*}\right)}^{\sigma \circ \lambda} \rightarrow \mathrm{M}_{\omega \circ \mu, \widehat{\omega}\left(U^{*}\right)}^{\omega \circ \lambda} ;
$$

see (3.2). This map also commutes with the actions of $\mathrm{A}^{\mathrm{G}}$ on the source and the target vector spaces. Note that $\widehat{\tau}_{m} \in \mathrm{O}_{2 m}$ is an involution, and the corresponding map (4.53) is invertible. Take the composition of the latter map with the map (3.15) determined by $\check{\xi}_{\sigma}$.

Thus for $\mathfrak{g}=\mathfrak{s p}_{2 m}$ or $\mathfrak{g}=\mathfrak{s o}_{2 m}$ and for any $\omega \in \mathfrak{R}$ we get a linear map

$$
\mathrm{M}_{\mu}^{\lambda} \rightarrow \mathrm{M}_{\omega \circ \mu, \widehat{\omega}\left(U^{*}\right)}^{\omega \circ \lambda},
$$

commuting with the actions of the algebra $\mathrm{A}^{\mathrm{G}}$ on the source and target vector spaces. Hence this is an intertwining operator of $\mathrm{X}\left(\mathfrak{g}^{\prime}\right)$-modules. By replacing the modules in (4.54) by their equivalents, and by using the observation that the series $f(x)$ for both modules is the same, we get an intertwining operator of $\mathrm{X}\left(\mathfrak{g}^{\prime}\right)$-modules

$$
\Phi_{\mu_{1}+\rho_{1}+\frac{1}{2}}^{\nu_{1}} \otimes \cdots \otimes \Phi_{\mu_{m}+\rho_{m}+\frac{1}{2}}^{\nu_{m}} \rightarrow \Phi_{\breve{\mu}_{1}+\breve{\rho}_{1}+\frac{1}{2}}^{\delta_{1} \breve{L}_{1}} \otimes \cdots \otimes \Phi_{\breve{\mu}_{m}+\breve{\rho}_{m}+\frac{1}{2}}^{\delta_{m} \breve{\nu}_{m}}
$$

The last two can also be regarded as $\mathrm{Y}\left(\mathfrak{g}^{\prime}\right)$-modules; our operator intertwines them. In this case we first take the tensor products of $Y\left(\mathfrak{g l}_{n}\right)$-modules, and then restrict both tensor products to the subalgebra $\mathrm{Y}\left(\mathfrak{g}^{\prime}\right) \subset \mathrm{Y}\left(\mathfrak{g l}_{n}\right)$. 
We will now give an analogue of Proposition 4.4 for $\mathrm{G}=\mathrm{Sp}_{2 m}, \mathrm{O}_{2 m}$. Arrange the indices $1, \ldots, n$ into the sequence

$$
1,3, \ldots, n-1, n, \ldots, 4,2 \quad \text { or } \quad 1,3, \ldots, n-2, n, n-1, \ldots, 4,2
$$

when $n$ is even or odd, respectively. The mapping $i \mapsto \tilde{\imath}$ reverses the sequence (4.56). We will write $i \prec j$ when $i$ precedes $j$ in this sequence. The elements $E_{i j}-\theta_{i} \theta_{j} E_{\tilde{\jmath} \tilde{}} \in \mathfrak{g l}_{n}$ with $i \prec j$ or $i=j$ span a Borel subalgebra of $\mathfrak{g}^{\prime} \subset \mathfrak{g l}_{n}$, while the elements $E_{i i}-E_{\tilde{\imath} \tilde{\imath}}$ span the corresponding Cartan subalgebra of $\mathfrak{g}^{\prime}$. Choose a vector $\psi_{k} \in \Phi^{k}$ as follows. For $\theta=1$ put $\psi_{k}=f_{1}^{k}$, so that $\varphi_{k}=\psi_{k}$ in this case. However, for $\theta=-1$, let $\psi_{k}$ be the exterior product of the vectors $f_{i}$ taken over the first $k$ indices in the sequence (4.56). For instance, $\psi_{2}=f_{1} \wedge f_{3}$ if $n \geqslant 3$. Note that the vector $\psi_{k}$ is annihilated by the action of the elements $E_{i j} \in \mathfrak{g l}_{n}$ with $i \prec j$.

For each positive root $\alpha$ of $\mathfrak{g}=\mathfrak{s p}_{2 m}, \mathfrak{s o}_{2 m}$, define $z_{\alpha} \in \mathbb{C}$ as follows. If $\theta=1$,

$$
z_{\alpha}= \begin{cases}\prod_{s=1}^{\nu_{b}} \frac{\mu_{a}-\mu_{b}+\rho_{a}-\rho_{b}-s}{\lambda_{a}-\lambda_{b}+\rho_{a}-\rho_{b}+s} & \text { if } \alpha=\eta_{m-b+1}-\eta_{m-a+1}, \\ \prod_{s=1}^{\nu_{b}} \frac{\mu_{a}+\mu_{b}+\rho_{a}+\rho_{b}+s}{\lambda_{a}+\lambda_{b}+\rho_{a}+\rho_{b}-s} & \text { if } \alpha=\eta_{m-b+1}+\eta_{m-a+1}, \\ \prod_{s=1}^{\left[\nu_{a} / 2\right]} \frac{\mu_{a}+\rho_{a}+s}{\lambda_{a}+\rho_{a}-s} & \text { if } \alpha=2 \eta_{m-a+1} .\end{cases}
$$

In the first two cases $1 \leqslant a<b \leqslant m$; in the third case $1 \leqslant a \leqslant m$ and $\mathfrak{g}=\mathfrak{s p}_{2 m}$. In the first case, the denominator corresponding to $s$ equals $(\lambda+\rho)\left(H_{\alpha}\right)+s$; in the last two cases the denominator equals $-(\lambda+\rho)\left(H_{\alpha}\right)-s$. All these denominators do not vanish for a dominant $\lambda+\rho$. If $\theta=-1$, then $z_{\alpha}=z_{\alpha}^{\prime} z_{\alpha}^{\prime \prime}$ where

$$
\begin{aligned}
& z_{\alpha}^{\prime}= \begin{cases}(-1)^{\nu_{a} \nu_{b}} & \text { if } \alpha=\eta_{m-b+1}-\eta_{m-a+1} \\
1 & \text { otherwise }\end{cases} \\
& z_{\alpha}^{\prime \prime}= \begin{cases}\frac{\lambda_{a}-\lambda_{b}+\rho_{a}-\rho_{b}}{\mu_{a}-\mu_{b}+\rho_{a}-\rho_{b}} & \text { if } \alpha=\eta_{m-b+1}-\eta_{m-a+1} \quad \text { and } \quad \nu_{a}<\nu_{b}, \\
\frac{\lambda_{a}+\lambda_{b}+\rho_{a}+\rho_{b}}{\mu_{a}+\mu_{b}+\rho_{a}+\rho_{b}} & \text { if } \alpha=\eta_{m-b+1}+\eta_{m-a+1} \quad \text { and } \quad \nu_{a}+\nu_{b}>n, \\
\frac{\lambda_{a}+\rho_{a}}{\mu_{a}+\rho_{a}} & \text { if } \alpha=2 \eta_{m-a+1} \quad \text { and } 2 \nu_{a}>n, \\
1 & \text { otherwise. }\end{cases}
\end{aligned}
$$

Here for $\theta=-1$ and all indices $a$, we have $\mu_{a}=\lambda_{a}-\nu_{a}+n / 2$. So in the first of the last four cases, the denominator equals $(\lambda+\rho)\left(H_{\alpha}\right)-\nu_{a}+\nu_{b}$. In the second of the four cases, the denominator equals $-(\lambda+\rho)\left(H_{\alpha}\right)-\nu_{a}-\nu_{b}+n$. In the third case, it equals $-(\lambda+\rho)\left(H_{\alpha}\right)-\nu_{a}+n / 2$. These denominators do not vanish for dominant $\lambda+\rho$, under the conditions they occur with. Denote by $\Delta^{++}$the set of compact positive roots of $\mathfrak{g}$, these are the weights $\eta_{a}-\eta_{b}$, where $1 \leqslant a<b \leqslant m$. 
Proposition 4.6. Let $\lambda+\rho$ be dominant. The operator (4.55) determined by $\check{\xi}_{\sigma}$ maps

$$
\psi_{\nu_{1}} \otimes \cdots \otimes \psi_{\nu_{m}} \in \Phi_{\mu_{1}+\rho_{1}+\frac{1}{2}}^{\nu_{1}} \otimes \cdots \otimes \Phi_{\mu_{m}+\rho_{m}+\frac{1}{2}}^{\nu_{m}}
$$

to the vector $\psi_{\breve{\nu}_{1}} \otimes \cdots \otimes \psi_{\breve{\nu}_{m}}$ of (4.51) multiplied by those $z_{\alpha}$ where $\omega(\alpha) \notin \Delta^{+}$ while

$$
\alpha \in \begin{cases}\Delta^{++} & \text {if } \theta=1 \text { and } n>1 \\ \Delta^{+} & \text {otherwise. }\end{cases}
$$

Proof. For a generic weight $\mu$, this proposition has been proved in [KN3, Section 5] and [KN4, Section 5] when $\theta=1$ or $\theta=-1$, respectively. But when $\nu$ is fixed, our operator (4.55) depends on $\mu \in \mathfrak{t}^{*}$ continiously; see the proof of Corollary 3.6.

Our intertwining operator of $\mathrm{Y}\left(\mathfrak{g}^{\prime}\right)$-modules 4.55) has been defined for any element $\omega \in \mathfrak{R}$. By definition, the corresponding element $\bar{\omega} \in \mathfrak{S}_{m} \ltimes \mathbb{Z}_{2}^{m}$ is a certain permuation of the indices $-m, \ldots,-1,1, \ldots, m$. Now consider the special case when the permutation $\bar{\omega}$ only changes the sign of each of these indices, so that $\omega=\omega_{1} \cdots \omega_{m}$ in the notation introduced just before stating Proposition 4.5 . Thus we get an intertwining operator

$$
\Phi_{\mu_{1}+\rho_{1}+\frac{1}{2}}^{\nu_{1}} \otimes \cdots \otimes \Phi_{\mu_{m}+\rho_{m}+\frac{1}{2}}^{\nu_{m}} \rightarrow \Phi_{\mu_{1}+\rho_{1}+\frac{1}{2}}^{-\nu_{1}} \otimes \cdots \otimes \Phi_{\mu_{m}+\rho_{m}+\frac{1}{2}}^{-\nu_{m}}
$$

of $\mathrm{Y}\left(\mathfrak{g}^{\prime}\right)$-modules. It corresponds to the longest element $\omega=\sigma_{0}$ of the Weyl group $\mathfrak{S}$, if $\mathfrak{g}=\mathfrak{s p}_{2 m}$ or if $\mathfrak{g}=\mathfrak{s o}_{2 m}$ and $m$ is even. If $\mathfrak{g}=\mathfrak{s o}_{2 m}$ and $m$ is odd, then $\sigma_{0}=\omega_{2} \cdots \omega_{m}$ so that (4.58) corresponds to the element $\omega=\tau_{m} \sigma_{0}$ of the extended Weyl group $\mathfrak{R}$.

For $\mathfrak{g}=\mathfrak{s p}_{2 m}, \mathfrak{s o}_{2 m}$, the element $\omega_{2} \cdots \omega_{m} \in \mathfrak{R}$ gives the intertwining operator

$$
\Phi_{\mu_{1}+\rho_{1}+\frac{1}{2}}^{\nu_{1}} \otimes \cdots \otimes \Phi_{\mu_{m}+\rho_{m}+\frac{1}{2}}^{\nu_{m}} \rightarrow \Phi_{\mu_{1}+\rho_{1}+\frac{1}{2}}^{\nu_{1}} \otimes \Phi_{\mu_{2}+\rho_{2}+\frac{1}{2}}^{-\nu_{2}} \otimes \cdots \otimes \Phi_{\mu_{m}+\rho_{m}+\frac{1}{2}}^{-\nu_{m}}
$$

of $\mathrm{Y}\left(\mathfrak{g}^{\prime}\right)$-modules. The underlying spaces of the two $\mathrm{Y}\left(\mathfrak{g l}_{n}\right)$-modules appearing as the first tensor factors on the right-hand sides of (4.58) and (4.59) are the same by definition. Moreover, for $\mathfrak{g}=\mathfrak{s o}_{2 m}$ our operators (4.58) and (4.59) are the same due to Proposition 4.5. In particular, for $\mathfrak{g}=\mathfrak{s o}_{2}$ the intertwining operator

$$
\Phi_{\mu_{1}+\rho_{1}+\frac{1}{2}}^{\nu_{1}} \rightarrow \Phi_{\mu_{1}+\rho_{1}+\frac{1}{2}}^{-\nu_{1}}
$$

corresponding to $\omega=\omega_{1}$ is the identity map. This explains the following fact from [N]. If $\mathfrak{g}^{\prime}=\mathfrak{s p}_{n}$ and $\theta=1$, or if $\mathfrak{g}^{\prime}=\mathfrak{s o}_{n}$ and $\theta=-1$, that is, if $\mathfrak{g}=\mathfrak{s o}_{2 m}$ in the context of the present article, then for any $t \in \mathbb{C}$ the restriction of the $\mathrm{Y}\left(\mathfrak{g l}_{n}\right)$ module $\Phi_{t}^{-k}$ to the subalgebra $\mathrm{Y}\left(\mathfrak{g}^{\prime}\right) \subset \mathrm{Y}\left(\mathfrak{g l}_{n}\right)$ coincides with the restriction of the $\mathrm{Y}\left(\mathfrak{g l}_{n}\right)$-module $\Phi_{t}^{k}$.

Now suppose that $\mathfrak{g}=\mathfrak{s p}_{2 m}$. Then we have $\mathfrak{R}=\mathfrak{S}$, so that $\mathfrak{R}_{\lambda}=\mathfrak{S}_{\lambda}$ for any $\lambda \in \mathfrak{t}^{*}$ automatically. Thus for any $\mu$ and dominant $\lambda+\rho$, Corollary 3.9 and Proposition 4.2 imply that the quotient by the kernel of our intertwining operator (4.58) is an irreducible $\mathrm{Y}\left(\mathfrak{g}^{\prime}\right)$-module. Here we also use the observation that any element of the subalgebra $\mathrm{U}\left(\mathfrak{s p}_{2 m}\right)^{\mathrm{Sp}_{2 m}} \otimes 1 \subset \mathrm{A}^{\mathrm{Sp}_{2 m}}$ acts on the subspace (4.41) via scalar multiplication. Note that here $\mathfrak{g}^{\prime}=\mathfrak{s} \mathfrak{o}_{n}$ or $\mathfrak{g}^{\prime}=\mathfrak{s p}_{n}$, respectively, for $\theta=1$ or $\theta=-1$. In the next section we will show that up to equivalence and similarity, any finite-dimensional irreducible $\mathrm{Y}\left(\mathfrak{s p}_{n}\right)$-module arises as the image of (4.58) for $\theta=-1$ and some $\lambda, \mu$. 
Next suppose that $\mathfrak{g}=\mathfrak{s o}_{2 m}$. Then $\mathfrak{R} \neq \mathfrak{S}$. Here for any $\mu$ and dominant $\lambda+\rho$, Corollary 3.9 and Proposition 4.2 imply that the quotient by the kernel, or equivalently the image of our intertwining operator (4.58) is an irreducible $\mathrm{Y}\left(\mathfrak{g}^{\prime}\right)$ module, under the extra condition that $\mathfrak{R}_{\lambda}=\mathfrak{S}_{\lambda}$. We also use the fact that any element of the subalgebra $\mathrm{U}\left(\mathfrak{s o}_{2 m}\right)^{\mathrm{O}_{2 m}} \otimes 1 \subset \mathrm{A}^{\mathrm{O}_{2 m}}$ acts on the subspace (4.41) via scalar multiplication. In the next subsection we study the quotient by the kernel of (4.58) without imposing that extra condition, but for $\theta=-1$ only. We will show that then the quotient is either an irreducible $\mathrm{Y}\left(\mathfrak{s o}_{n}\right)$-module or splits into a direct sum of two nonequivalent irreducible $\mathrm{Y}\left(\mathfrak{s o}_{n}\right)$-modules. In Section 5 we will explain which irreducible $\mathrm{Y}\left(\mathfrak{s o}_{n}\right)$-modules arise in this particular way.

4.5. Crossed product algebras. For any pair $\left(\mathrm{G}, \mathrm{G}^{\prime}\right)$ take the crossed product algebra $G^{\prime} \ltimes A$. The action of $G^{\prime}$ on $A$ commutes with that of the group $G$, and leaves invariant any element of the subalgebra $U(\mathfrak{g}) \subset A$. Therefore the group $G^{\prime}$ acts by automorphisms of the double coset algebra $\bar{Z}$, and we can also define the crossed product algebra $G^{\prime} \ltimes \bar{Z}$.

The action of $\mathrm{G}^{\prime}$ on $\overline{\mathrm{Z}}$ commutes with the Zhelobenko automorphisms $\check{\xi}_{1}, \ldots, \check{\xi}_{r}$. Since it also commutes with the action of $\widehat{\tau} \in \mathrm{G}$ on $\overline{\mathrm{Z}}$ for any $\tau \in \mathfrak{T}$, the action of $\mathrm{G}^{\prime}$ preserves the subalgebra $\mathrm{Q} \subset \overline{\mathrm{Z}}$. So we get the crossed product algebra $\mathrm{G}^{\prime} \ltimes \mathrm{Q}$. The homomorphism $\mathrm{U}\left(\mathfrak{g}^{\prime}\right) \rightarrow \mathrm{A}^{\mathrm{G}}$ used in Subsection 4.4 yields a homomorphism $\mathrm{U}\left(\mathfrak{g}^{\prime}\right) \rightarrow \mathrm{Q}$.

For any $\lambda, \mu \in \mathfrak{t}^{*}$ the subspace $\mathrm{M}_{\mu}^{\lambda} \subset \mathrm{M}_{\mu}$ is a module over the subalgebra

$$
\mathrm{G}^{\prime} \ltimes \mathrm{A}^{\mathrm{G}} \subset \mathrm{G}^{\prime} \ltimes \mathrm{A},
$$

and moreover an $\left(\mathrm{A}^{\mathrm{G}}, \mathrm{G}^{\prime}\right)$-module. If $\lambda+\rho$ is dominant, the subspace $\mathrm{M}_{\mu}^{\lambda} \subset \mathrm{M}_{\mu}$ is also a $\mathrm{Q}$-module, and moreover a $\left(\mathrm{Q}, \mathrm{G}^{\prime}\right)$-module. Then the action of $\mathrm{A}^{\mathrm{G}}$ on $\mathrm{M}_{\mu}^{\lambda}$ can also be obtained by pulling the action of $\mathrm{Q}$ on $\mathrm{M}_{\mu}^{\lambda}$ back through the isomorphism $\gamma: \mathrm{A}^{\mathrm{G}} \rightarrow \mathrm{Q}$; see Subsection 3.3. Moreover, the isomorphism $\gamma$ is $\mathrm{G}^{\prime}$-equivariant. Hence by using $\gamma$ we get the same structure of an $\left(\mathrm{A}^{\mathrm{G}}, \mathrm{G}^{\prime}\right)$-module on $\mathrm{M}_{\mu}^{\lambda}$ as above.

The Zhelobenko operator $\check{\xi}_{0}$ on $\overline{\mathrm{A}} / \overline{\mathrm{J}}$ is $\mathrm{G}^{\prime}$-equivariant. So is the corresponding linear map (3.16) for any $\mu$ and dominant $\lambda+\rho$. Hence the action of the group $\mathrm{G}^{\prime}$ preserves the subspace $\operatorname{Ker}\left(\check{\xi}_{0} \mid \mathrm{M}_{\mu}^{\lambda}\right)$ of $\mathrm{M}_{\mu}^{\lambda}$. Another way to see this is to use Proposition 3.7 and $\mathrm{G}^{\prime}$-contravariance of the Shapovalov form $S_{\mu}^{\lambda}$ on $\mathrm{M}_{\mu}^{\lambda}$. The latter property means that

$$
S_{\mu}^{\lambda}(v(f), g)=S_{\mu}^{\lambda}\left(f, v^{\prime}(g)\right) \text { for } \quad f, g \in \mathrm{M}_{\mu}^{\lambda},
$$

where $v \mapsto v^{\prime}$ is the anti-involution on $\mathrm{G}^{\prime}$ defined by the matrix transposition. Here the elements of $\mathrm{G}^{\prime}$ are regarded as matrices by using the standard basis $f_{1}, \ldots, f_{n}$ of $\mathbb{C}^{n}$. Either way, the quotient space of $\mathrm{M}_{\mu}^{\lambda}$ by $\operatorname{Ker}\left(\check{\xi}_{0} \mid \mathrm{M}_{\mu}^{\lambda}\right)$ becomes a $\left(\mathrm{Q}, \mathrm{G}^{\prime}\right)$ module.

When the Lie group $\mathrm{G}^{\prime}$ is connected, that is, when $\mathrm{G}^{\prime}=\mathrm{GL}_{n}$ or $\mathrm{G}^{\prime}=\mathrm{Sp}_{n}$, we will not need to use the action of $\mathrm{G}^{\prime} \ltimes \mathrm{Q}$ on $\mathrm{M}_{\mu}^{\lambda}$. It will suffice to use only the action of Q. But the Lie group $\mathrm{G}^{\prime}=\mathrm{O}_{n}$ is not connected. Until the end of this subsection we will be considering only the case when $\left(\mathrm{G}, \mathrm{G}^{\prime}\right)=\left(\mathrm{O}_{2 m}, \mathrm{O}_{n}\right)$ so that $\theta=-1$. The space $U$ has been identified with the tensor product $\mathbb{C}^{m} \otimes \mathbb{C}^{n}$. In our case $\mathrm{H}(U)$ is the Clifford algebra generated by the elements of the vector space $W=\mathbb{C}^{2 m} \otimes \mathbb{C}^{n}$ subject to the relations (2.2), where $B$ is the tensor product of the symmetric forms on $\mathbb{C}^{2 m}$ and $\mathbb{C}^{n}$ preserved by the actions of the groups $\mathrm{O}_{2 m}$ and 
$\mathrm{O}_{n}$, respectively. Here $x_{a i}=e_{a} \otimes f_{i}$ and $\partial_{a i}=e_{-a} \otimes f_{\tilde{\imath}}$ for $a=1, \ldots, m$ and for all $i=1, \ldots, n$. Note that $\theta_{i}=1$ in this case.

Choose any vector $f_{0} \in \mathbb{C}^{n}$ of length $\sqrt{2}$ with respect to the form preserved by $\mathrm{O}_{n}$. The corresponding orthogonal reflection is

$$
v_{0}: \mathbb{C}^{n} \rightarrow \mathbb{C}^{n}: u \mapsto u-z f_{0},
$$

where $z$ is the value of the symmetric form on $\mathbb{C}^{n}$ taken on the pair of vectors $\left(f_{0}, u\right)$. This reflection is an element of the group $\mathrm{O}_{n}$. It defines an automorphism of the algebra $\mathrm{H}(U)$, which preserves the subalgebra $\mathrm{P}(U)$ generated by all elements $x_{a i}$.

For $a=1, \ldots, m$ define the vectors $x_{a}=e_{a} \otimes f_{0}$ and $\partial_{a}=e_{-a} \otimes f_{0}$ of $\mathbb{C}^{2 m} \otimes \mathbb{C}^{n}$. By (2.2) we get

$$
x_{a} \partial_{a}+\partial_{a} x_{a}=B\left(x_{a}, \partial_{a}\right)=2 .
$$

Consider the product

$$
A_{0}=\prod_{a=1}^{m}\left(1-x_{a} \partial_{a}\right) \in \mathrm{H}(U) .
$$

The $m$ factors here commute pairwise. We will use the next properties of $A_{0}$.

Lemma 4.7. (i) There is an equality $\widehat{\tau}_{m}\left(A_{0}\right)=-A_{0}$.

(ii) The action of the element $A_{0}$ on $\mathrm{P}(U)$ coincides with that of the reflection $v_{0}$.

(iii) The element $A_{0} \in \mathrm{H}(U)$ commutes with $\zeta(X)$ for every $X \in \mathfrak{s o}_{2 m}$.

Proof. In our case, the action of the element $\widehat{\tau}_{m} \in \mathrm{O}_{2 m}$ on $\mathbb{C}^{2 m}$ exchanges $e_{1}$ with $e_{-1}$ and leaves other basis vectors of $\mathbb{C}^{2 m}$ fixed. So the action of $\widehat{\tau}_{m}$ on $\mathrm{H}(U)$ exchanges the element $x_{1}$ with $\partial_{1}$, and leaves fixed any element $x_{a}$ or $\partial_{a}$ with $a>1$. Now (i) follows from the relation (4.60) with $a=1$. Part (ii) can be obtained by a direct calculation, which reduces to the case $m=1$. Part (iii) follows from (ii), because every element $\zeta(X) \in \mathrm{H}(U)$ with $X \in \mathfrak{s o}_{2 m}$ is $\mathrm{O}_{n}$-invariant.

Proposition 4.8. Suppose that $\lambda+\rho$ is dominant and $\left(\mathrm{G}, \mathrm{G}^{\prime}\right)=\left(\mathrm{O}_{2 m}, \mathrm{O}_{n}\right)$. Then for any $\mu$ the quotient $\mathrm{M}_{\mu}^{\lambda} / \operatorname{Ker}\left(\check{\xi}_{0} \mid \mathrm{M}_{\mu}^{\lambda}\right)$ is an irreducible $\left(\mathrm{Q}, \mathrm{O}_{n}\right)$-module.

Proof. Due to Propositions 2.6 and 3.7, the quotient $N=\mathrm{M}_{\mu}^{\lambda} / \operatorname{Ker}\left(\check{\xi}_{0} \mid \mathrm{M}_{\mu}^{\lambda}\right)$ is an irreducible S-module. By applying Proposition [1.6 to this S-module, we get Proposition 4.8 in the case when $\mathfrak{R}_{\lambda}=\mathfrak{S}_{\lambda}$. In this case Proposition 4.8 follows from Theorem 3.8. We will now alter our proof of Proposition 1.6, to get Proposition 4.8 without assuming that $\mathfrak{R}_{\lambda}=\mathfrak{S}_{\lambda}$.

Instead of the element (1.23) of the algebra Q, first consider an element of S,

$$
Z^{\prime}=\left|\mathfrak{S}_{\lambda}\right|^{-1} \sum_{\sigma \in \mathfrak{S}} \check{\xi}_{\sigma}\left(X^{\prime} Y\right)
$$

Here $X^{\prime}$ and $Y$ are the same as in (1.23), but the sum is taken over the Weyl group $\mathfrak{S}$. The arguments given just after (1.23) show that $\chi(Y)=\chi\left(Z^{\prime}\right)$. We also get the equality $\check{\xi}_{\sigma}\left(Z^{\prime}\right)=Z^{\prime}$ for every $\sigma \in \mathfrak{S}$. But we might have $\widehat{\tau}_{m}\left(Z^{\prime}\right) \neq Z^{\prime}$, so that then $Z^{\prime} \notin \mathrm{Q}$.

Then put $Z=Z^{\prime}+\widehat{\tau}_{m}\left(Z^{\prime}\right)$. Since $\widehat{\tau}_{m}^{2}=1$, we get the equality $\widehat{\tau}_{m}(Z)=Z$. The action of the element $\widehat{\tau}_{m} \in \mathrm{O}_{2 m}$ on $\mathrm{S}$ exchanges the operator $\check{\xi}_{m-1}$ with $\check{\xi}_{m}$, and commutes with the operators $\check{\xi}_{1}, \ldots, \check{\xi}_{m-2}$. Hence $\check{\xi}_{\sigma}(Z)=Z$ for any $\sigma \in \mathfrak{S}$. Thus we have $Z \in \mathrm{Q}$, as we had for the element (1.23). 
Now put $Z^{\prime \prime}=Z^{\prime}-Z$. Then $\check{\xi}_{\sigma}\left(Z^{\prime \prime}\right)=Z^{\prime \prime}$ for any $\sigma \in \mathfrak{S}$, while $\widehat{\tau}_{m}\left(Z^{\prime \prime}\right)=$ $-Z^{\prime \prime}$. But here we have $Z^{\prime \prime} A_{0} \in \mathrm{Q}$. Indeed, by part (iii) of Lemma 4.7 we have $\check{\xi}_{\sigma}\left(Z^{\prime \prime} A_{0}\right)=Z^{\prime \prime} A_{0}$ for any $\sigma \in \mathfrak{S}$, while $\widehat{\tau}_{m}\left(Z^{\prime \prime} A_{0}\right)=Z^{\prime \prime} A_{0}$ by part (i). Now consider the element

$$
Z^{\prime \prime} A_{0} v_{0}^{-1} \in \mathrm{O}_{n} \ltimes \mathrm{Q} .
$$

Its action on $N$ coincides with that of the element $Z^{\prime \prime}$ by part (ii) of Lemma 4.7 Hence the action of

$$
Z+Z^{\prime \prime} A_{0} v_{0}^{-1} \in \mathrm{O}_{n} \ltimes \mathrm{Q}
$$

coincides with that of the element $Z^{\prime}$. However, the latter action coincides with that of the element $Y$.

Corollary 4.9. Let $\lambda+\rho$ be dominant and $\left(\mathrm{G}, \mathrm{G}^{\prime}\right)=\left(\mathrm{O}_{2 m}, \mathrm{O}_{n}\right)$, so that $\theta=-1$.

(i) If $n$ is odd, then the quotient $\mathrm{M}_{\mu}^{\lambda} / \operatorname{Ker}\left(\check{\xi}_{0} \mid \mathrm{M}_{\mu}^{\lambda}\right)$ is an irreducible $\mathrm{A}^{\mathrm{O}_{2 m}}$ module.

(ii) If $n$ is even, then $\mathrm{M}_{\mu}^{\lambda} / \operatorname{Ker}\left(\check{\xi}_{0} \mid \mathrm{M}_{\mu}^{\lambda}\right)$ is either an irreducible $\mathrm{A}^{\mathrm{O}_{2 m}}$-module or splits into a direct sum of two irreducible nonequivalent $\mathrm{A}^{\mathrm{O}_{2 m}}$-modules.

Proof. If $n$ is odd, then the group $\mathrm{O}_{n}$ splits as a direct product $\mathbb{Z}_{2} \times \mathrm{SO}_{n}$ where $\mathbb{Z}_{2}$ is the subgroup of $\mathrm{O}_{n}$ generated by the minus identity element. This element acts on $\mathrm{M}_{\mu}^{\lambda}$ as the multiplication by $(-1)^{\nu_{1}+\cdots+\nu_{m}}$. Instead of the action of the connected Lie group $\mathrm{SO}_{n}$ on $\mathrm{M}_{\mu}^{\lambda}$, it suffices to consider the action of the Lie algebra $\mathfrak{s o}_{n}$. But the latter action can also be obtained by pulling the action of $\mathrm{A}^{\mathrm{O}_{2 m}}$ on $\mathrm{M}_{\mu}^{\lambda}$ back through the homomorphism $\mathrm{U}\left(\mathfrak{s o}_{n}\right) \rightarrow \mathrm{A}^{\mathrm{O}_{2 m}}$, as mentioned earlier in this subsection. Proposition 1.5 now yields (i).

When $n$ is even, we can use the action of the Lie algebra $\mathfrak{s o}_{n}$ on $\mathrm{M}_{\mu}^{\lambda}$ instead of the action of the subgroup $\mathrm{SO}_{n} \subset \mathrm{O}_{n}$. This is a normal subgroup of index two. We can also use the homomorphism $\mathrm{U}\left(\mathfrak{s o}_{n}\right) \rightarrow \mathrm{A}^{\mathrm{O}_{2 m}}$, as we did for an odd $n$. Proposition 1.5 now implies that the quotient $\mathrm{M}_{\mu}^{\lambda} / \operatorname{Ker}\left(\check{\xi}_{0} \mid \mathrm{M}_{\mu}^{\lambda}\right)$ is irreducible over the joint action of $\mathrm{A}^{\mathrm{O}_{2 m}}$ and of any element $v$ from the complement to $\mathrm{SO}_{n}$ in $\mathrm{O}_{n}$. Now general arguments from [W, Section V.8] yield (ii). Moreover, if the quotient is a direct sum of two irreducible $\mathrm{A}^{\mathrm{O}_{2 m}}$-modules and $N$ is one of them, the space of the other equals $v N$ for any $v$.

4.6. Equivalent modules. Let $\left(\mathrm{G}, \mathrm{G}^{\prime}\right)$ be any dual pair from Subsection 4.1. For any weights $\lambda$ and $\mu$ of $\mathfrak{g}$, the subspace (2.31) can be regarded as an $\mathrm{A}^{\mathrm{G}}$-module. We have denoted the subspace by $\mathrm{L}_{\mu}^{\lambda}$. First consider $\left(\mathrm{G}, \mathrm{G}^{\prime}\right)=\left(\mathrm{GL}_{m}, \mathrm{GL}_{n}\right)$. By using the homomorphism $\mathrm{Y}\left(\mathfrak{g l}_{n}\right) \rightarrow \mathrm{A}^{\mathrm{GL}_{m}}$, we can also regard $\mathrm{L}_{\mu}^{\lambda}$ as an $\mathrm{Y}\left(\mathfrak{g l}_{n}\right)$-module. By Corollary 2.8 the $\mathrm{Y}\left(\mathfrak{g l}_{n}\right)$-module $\mathrm{L}_{\mu}^{\lambda}$ is irreducible for any $\mu$ and dominant $\lambda+\rho$. Here we also use Propositions 1.5 and 4.1 .

Suppose that the weight $\nu=\lambda-\mu$ satisfies the conditions of Proposition 4.3 , Using that proposition, the $\mathrm{Y}\left(\mathfrak{g l}_{n}\right)$-module $\mathrm{L}_{\mu}^{\lambda}$ is equivalent to a certain quotient of the tensor product (4.46). The latter tensor product can be regarded as a subspace in $\mathrm{P}(U)$. Denote by $u$ the vector of $\mathrm{P}(U)$ corresponding to the vector $\varphi_{\nu_{1}} \otimes \cdots \otimes \varphi_{\nu_{m}}$ of (4.46). If the weight $\lambda+\rho$ is dominant, then $\zeta(P[\mu+\rho]) u=z u$ where $z$ stands for the product of all $z_{\alpha}$ with $\alpha \in \Delta^{+}$. This equality follows from Propositions 3.5 and 4.4. It can also be obtained directly from [KN1, Section 3] or [KN2, Section 3] when $\theta=1$ or $\theta=-1$, respectively. If $z \neq 0$, then by Propositions 2.9 and 3.7 the 
$\mathrm{Y}\left(\mathfrak{g l}_{n}\right)$-module $\mathrm{L}_{\mu}^{\lambda}$ is equivalent to the image of our intertwining operator (4.49). Then $\mathrm{L}_{\mu}^{\lambda}$ is not zero.

Now take any pair $\left(\mathrm{G}, \mathrm{G}^{\prime}\right)$ from Subsection 4.1 other than $\left(\mathrm{GL}_{m}, \mathrm{GL}_{n}\right)$. By using the homomorphism $\mathrm{X}\left(\mathfrak{g}^{\prime}\right) \rightarrow \mathrm{A}^{\mathrm{G}}$ we can regard $\mathrm{L}_{\mu}^{\lambda}$ as an $\mathrm{X}\left(\mathfrak{g}^{\prime}\right)$-module. Moreover, $\mathrm{L}_{\mu}^{\lambda}$ can be regarded as an $\left(\mathrm{X}\left(\mathfrak{g}^{\prime}\right), \mathrm{G}^{\prime}\right)$-module; see Subsection 4.5. Take any $\mu$ and dominant $\lambda+\rho$. By Corollary 2.8 the $\mathrm{X}\left(\mathfrak{g}^{\prime}\right)$-module $\mathrm{L}_{\mu}^{\lambda}$ is irreducible if $\mathfrak{R}_{\lambda}=\mathfrak{S}_{\lambda}$. Here we also use Propositions 1.5 and 4.2. Next take $\left(\mathrm{G}, \mathrm{G}^{\prime}\right)=$ $\left(\mathrm{O}_{2 m}, \mathrm{O}_{n}\right)$, so that $\theta=-1$. Our proof of Proposition 4.8 demonstrates that $\mathrm{L}_{\mu}^{\lambda}$ is an irreducible $\left(\mathrm{X}\left(\mathfrak{s o}_{n}\right), \mathrm{O}_{n}\right)$-module. Our proof of Corollary 4.9 demonstrates that $\mathrm{L}_{\mu}^{\lambda}$ is an irreducible $\mathrm{X}\left(\mathfrak{s o}_{n}\right)$-module if $n$ is odd. If $n$ is even, then $\mathrm{L}_{\mu}^{\lambda}$ is either an irreducible $\mathrm{X}\left(\mathfrak{s o}_{n}\right)$-module, or is a direct sum of two irreducible nonequivalent $\mathrm{X}\left(\mathfrak{s o}_{n}\right)$-modules.

Now consider again any pair $\left(\mathrm{G}, \mathrm{G}^{\prime}\right)$ from Subsection 4.1 other than $\left(\mathrm{GL}_{m}, \mathrm{GL}_{n}\right)$. Suppose that the weight $\nu=\lambda-\mu-\kappa$ satisfies the conditions of Proposition 4.5. Using that proposition in the case when $\omega$ is the identity element of the group $\mathfrak{R}$, the $\mathrm{X}\left(\mathfrak{g}^{\prime}\right)$-module $\mathrm{L}_{\mu}^{\lambda}$ is equivalent to a certain quotient of the tensor product of the form (4.46). This tensor product can be regarded as a subspace in $\mathrm{P}(U)$. Let $u$ be the vector of $\mathrm{P}(U)$ corresponding to (4.57). If $\lambda+\rho$ is dominant, then we again have the equality $\zeta(P[\mu+\rho]) u=z u$. But here $z$ stands for the product of all $z_{\alpha}$ with $\alpha \in \Delta^{++}$if $\theta=1$ and $n>1$. Otherwise, $z$ stands for the product of all $z_{\alpha}$ with $\alpha \in \Delta^{+}$. The equality follows from Propositions 3.5] and 4.6. It can also be obtained directly from [KN3, Erratum] or from [KN4, Section 5] when $\theta=1$ or $\theta=-1$, respectively. If $z \neq 0$, then by Propositions 2.9 and 3.7 the $\mathrm{X}\left(\mathfrak{g}^{\prime}\right)$-module $\mathrm{L}_{\mu}^{\lambda}$ is equivalent to the image of our intertwining operator (4.58). Moreover, then $\mathrm{L}_{\mu}^{\lambda}$ is equivalent to the image of (4.58) as an $\left(\mathrm{X}\left(\mathfrak{g}^{\prime}\right), \mathrm{G}^{\prime}\right)$-module. Note that then the quotient $\mathrm{L}_{\mu}^{\lambda}$ of $\mathrm{M}_{\mu}^{\lambda}$ is not zero; see Subsection 2.5.

4.7. Dual modules. In Subsection 3.4 for any $\mu$ and dominant $\lambda+\rho$, we defined a Q-contravariant pairing (3.19). Let us now describe that pairing in terms of representations of Yangians. First take $\left(\mathrm{G}, \mathrm{G}^{\prime}\right)=\left(\mathrm{GL}_{m}, \mathrm{GL}_{n}\right)$. The Chevalley anti-involution $\varepsilon$ on the Lie algebra $\mathfrak{g}=\mathfrak{g l}_{m}$ is defined by $\varepsilon\left(E_{a b}\right)=E_{b a}$. It extends to an involutive anti-automorphism of the algebra $\mathrm{A}$, also denoted by $\varepsilon$, so that the extension exchanges the generators $x_{a i}$ and $\partial_{a i}$ of the subalgebra $\mathrm{H}(U) \subset \mathrm{A}$. First applying the homomorphism $\mathrm{Y}\left(\mathfrak{g l}_{n}\right) \rightarrow$ A defined by (4.24) and then applying the anti-automorphism $\varepsilon$ of A amounts to first applying the anti-automorphism (4.11) of $\mathrm{Y}\left(\mathfrak{g l}_{n}\right)$ and then applying the homomorphism (4.24). Therefore, when we regard the two vector spaces in the pairing (3.19) as $\mathrm{Y}\left(\mathfrak{g l}_{n}\right)$-modules, the pairing becomes contravariant relative to the anti-automorphism (4.11) of $\mathrm{Y}\left(\mathfrak{g l}_{n}\right)$.

By replacing these two $\mathrm{Y}\left(\mathfrak{g l}_{n}\right)$-modules by their equivalents, we obtain a nondegenerate contravariant pairing of the source and target $\mathrm{Y}\left(\mathfrak{g l}_{n}\right)$-modules in (4.49). In particular, the $\mathrm{Y}\left(\mathfrak{g l}_{n}\right)$-module dual to the source in (4.49), is equivalent to the target. The latter equivalence can be proved directly, for all $\lambda$ and $\mu$. Indeed, because (4.11) is a coalgebra anti-automorphism, it is enough to take $m=1$ only. But the $\mathrm{Y}\left(\mathfrak{g l}_{n}\right)$-modules $\Phi_{t}^{k}$ are equivalent to their duals relative to (4.11), whenever $\theta=1$ or $\theta=-1$. See [NT1, Proposition 1.7] for a more general result.

Now let $\left(\mathrm{G}, \mathrm{G}^{\prime}\right)$ be any pair from (0.1) other than $\left(\mathrm{GL}_{m}, \mathrm{GL}_{n}\right)$. The Chevalley anti-involution $\varepsilon$ on the Lie algebra $\mathfrak{g}$ is defined for $a, b=-m, \ldots,-1,1, \ldots, m$ by

$$
\varepsilon\left(F_{a b}\right)=\operatorname{sign} a b \cdot F_{b a} \quad \text { or } \quad \varepsilon\left(F_{a b}\right)=F_{b a}
$$


when $\theta=1$ or $\theta=-1$, respectively. It extends to an involutive anti-automorphism of the algebra $\mathrm{A}$, also denoted by $\varepsilon$, so that the extension exchanges the element $p_{a i} \in \mathrm{H}(U)$ with

$$
-\operatorname{sign} a \cdot q_{a i} \text { or } q_{a i}
$$

when $\theta=1$ or $\theta=-1$, respectively. Here we use the notation from Proposition 4.2

First applying the homomorphism $\mathrm{X}\left(\mathfrak{g}^{\prime}\right) \rightarrow$ A defined by (4.33), and applying the anti-automorphism $\varepsilon$ of A after, amounts to first applying the anti-automorphism (4.20) of $\mathrm{X}\left(\mathfrak{g}^{\prime}\right)$ and then applying the homomorphism (4.33). Therefore, when we regard the two vector spaces in the pairing (3.19) as $\mathrm{X}\left(\mathfrak{g}^{\prime}\right)$-modules, the pairing becomes contravariant relative to the involutive anti-automorphism (4.20) of $\mathrm{X}\left(\mathfrak{g}^{\prime}\right)$.

By replacing the two $\mathrm{X}\left(\mathfrak{g}^{\prime}\right)$-modules by their equivalents, we get a nondegenerate contravariant pairing of the source and target $\mathrm{X}\left(\mathfrak{g}^{\prime}\right)$-modules in (4.58) if $\mathfrak{g}=\mathfrak{s p}_{2 m}$ or if $\mathfrak{g}=\mathfrak{s o}_{2 m}$ and $m$ is even. If $\mathfrak{g}=\mathfrak{s o}_{2 m}$ and $m$ is odd, then we get a pairing of the source and target $\mathrm{X}\left(\mathfrak{g}^{\prime}\right)$-modules in (4.59). But if $\mathfrak{g}=\mathfrak{s o}_{2 m}$, then the target $\mathrm{X}\left(\mathfrak{g}^{\prime}\right)$-modules in (4.58) and (4.59) are equivalent; see the end of Subsection 4.4. Thus for both $\mathfrak{g}=\mathfrak{s p}_{2 m}, \mathfrak{s o}_{2 m}$ and for any $m$ we get a nondegenerate contravariant pairing of the source and target $\mathrm{X}\left(\mathfrak{g}^{\prime}\right)$-modules in (4.58). Let us now regard them as $\mathrm{Y}\left(\mathfrak{g}^{\prime}\right)$-modules, as we did in Subsection 4.4. Then we get a nondegenerate pairing of them, which is contravariant relative to the restriction of the involutive anti-automorphism (4.19) of $\mathrm{Y}\left(\mathfrak{g l}_{n}\right)$ to the subalgebra $\mathrm{Y}\left(\mathfrak{g}^{\prime}\right) \subset \mathrm{Y}\left(\mathfrak{g l}_{n}\right)$. We also use the fact that any automorphism of $\mathrm{X}\left(\mathfrak{g}^{\prime}\right)$ defined by (4.15) commutes with the anti-automorphism defined by (4.20). In particular, the $\mathrm{Y}\left(\mathfrak{g}^{\prime}\right)$-module dual to the source in (4.58), is equivalent to the target.

As in the case $\mathfrak{g}^{\prime}=\mathfrak{g l}_{n}$, the equivalence here can be proved for all $\lambda$ and $\mu$ directly. Indeed, the $\mathrm{Y}\left(\mathfrak{g}^{\prime}\right)$-module dual to the source in (4.58) can be defined by first considering the source as a $\mathrm{Y}\left(\mathfrak{g l}_{n}\right)$-module, then taking its dual relative to the anti-automorphism (4.19) of $\mathrm{Y}\left(\mathfrak{g l}_{n}\right)$, and then restricting the resulting $\mathrm{Y}\left(\mathfrak{g l}_{n}\right)$ module to the subalgebra $\mathrm{Y}\left(\mathfrak{g}^{\prime}\right) \subset \mathrm{Y}\left(\mathfrak{g l}_{n}\right)$. But (4.19) is the composition of (4.11) and (4.12). So taking the dual relative to (4.19) amounts to first taking the dual relative to (4.11) and then pulling the result back through the automorphism (4.12).

We have already proved that relative to (4.11), the $\mathrm{Y}\left(\mathfrak{g l}_{n}\right)$-module dual to the source tensor product in (4.58) is equivalent to the tensor product of the same factors, but taken in the reversed order. Pulling the latter tensor product back through (4.12) replaces each factor $\Phi_{t}^{k}$ by $\Phi_{t}^{-k}$, and also reverses the order of the factors once again. Thus we get the target in (4.58) as $\mathrm{Y}\left(\mathfrak{g l}_{n}\right)$-module. Here we used the definition of the $\mathrm{Y}\left(\mathfrak{g l}_{n}\right)$-module $\Phi_{t}^{-k}$, and the fact that (4.12) defines an anti-automorphism of the coalgebra $\mathrm{Y}\left(\mathfrak{g l}_{n}\right)$.

\section{IrREdUCiBle REPRESENTATIONS OF YANGIANS}

5.1. Irreducible representations of $\mathrm{Y}\left(\mathfrak{g l}_{n}\right)$. Let $\Phi$ be an arbitrary nonzero finite-dimensional $\mathrm{Y}\left(\mathfrak{g l}_{n}\right)$-module. A nonzero vector of $\Phi$ is called highest if it is annihilated by all the coefficients of the series $T_{i j}(x)$ with $i<j$. If $\Phi$ is irreducible, then a highest vector $\varphi \in \Phi$ is unique up to a scalar multiplier. Moreover, then $\varphi$ is an eigenvector for the coefficients of all series $T_{i i}(x)$, and for $i=1, \ldots, n-1$,

$$
T_{i i}(x) T_{i+1, i+1}(x)^{-1} \varphi=P_{i}\left(x+\frac{1}{2}\right) P_{i}\left(x-\frac{1}{2}\right)^{-1} \varphi,
$$

where $P_{i}(x)$ is a monic polynomial in $x$ with coefficients in $\mathbb{C}$. Then polynomials $P_{1}(x), \ldots, P_{n-1}(x)$ are called the Drinfeld polynomials of $\Phi$. Any sequence of $n-1$ 
monic polynomials with complex coefficients arises in this way. Furthermore, two irreducible finite-dimensional $\mathrm{Y}\left(\mathfrak{g l}_{n}\right)$-modules have the same Drinfeld polynomials if and only if their restrictions to the subalgebra $S Y\left(\mathfrak{g l}_{n}\right) \subset \mathrm{Y}\left(\mathfrak{g l}_{n}\right)$ are equivalent. Thus up to equivalence and similarity, all the nonzero irreducible finite-dimensional $\mathrm{Y}\left(\mathfrak{g l}_{n}\right)$-modules are parametrized by their Drinfeld polynomials D2, Theorem 2]. For example, consider the trivial $\mathrm{Y}\left(\mathfrak{g l}_{n}\right)$-module. This module is one dimensional, and is defined by the counit homomorphism $\mathrm{Y}\left(\mathfrak{g l}_{n}\right) \rightarrow \mathbb{C}$. Then the corresponding Drinfeld polynomials are also trivial: $P_{1}(x)=\cdots=P_{n-1}(x)=1$.

In this subsection we will assume that $\theta=-1$ and $\mathfrak{g}=\mathfrak{g l}_{m}$, so that $\mathfrak{g}^{\prime}=\mathfrak{g l}_{n}$. For any $k \in\{0,1, \ldots, n\}$ the vector space $\Phi^{k}$ is irreducible under the action of $\mathfrak{g l}_{n}$. Hence for any $t \in \mathbb{C}$ the $\mathrm{Y}\left(\mathfrak{g l}_{n}\right)$-module $\Phi_{t}^{k}$ is irreducible. The vector $\varphi_{k}=$ $f_{1} \wedge \cdots \wedge f_{k}$ of this module is highest; see the definitions (4.6) and (4.8). Moreover, by these definitions

$$
T_{i i}(x) \varphi_{k}=\left\{\begin{array}{ccc}
(x-t+1)(x-t)^{-1} \varphi_{k} & \text { if } & 1 \leqslant i \leqslant k \\
\varphi_{k} & \text { if } & k<i \leqslant n .
\end{array}\right.
$$

So for $i=1, \ldots, n-1$ the Drinfeld polynomial $P_{i}(x)$ of the $\mathrm{Y}\left(\mathfrak{g l}_{n}\right)$-module $\Phi_{t}^{k}$ is

$$
P_{i}(x)=\left\{\begin{array}{cl}
x-t+\frac{1}{2} & \text { if } i=k ; \\
1 & \text { otherwise. }
\end{array}\right.
$$

For $\mathfrak{g}=\mathfrak{g l}_{m}$ we have $\nu=\lambda-\mu$. Suppose that each label $\nu_{a} \in\{0,1, \ldots, n\}$. Further suppose that the weight $\lambda+\rho$ is dominant. The latter condition means here that

$$
\lambda_{b}-\lambda_{a}+\rho_{b}-\rho_{a} \neq 1,2, \ldots \text { for all } 1 \leqslant a<b \leqslant m .
$$

By Corollary 3.9 and Proposition 4.1, the quotient by the kernel of our operator (4.49) is then an irreducible $\mathrm{Y}\left(\mathfrak{g l}_{n}\right)$-module. But the definition (4.10) implies that the vector $\varphi_{\nu_{1}} \otimes \cdots \otimes \varphi_{\nu_{m}}$ of (4.46) is highest. Suppose that

$$
\nu_{a} \geqslant \nu_{b} \quad \text { whenever } \quad \lambda_{a}+\rho_{a}=\lambda_{b}+\rho_{b} \quad \text { and } \quad a<b .
$$

Due to the dominance of the weight $\lambda+\rho$, Proposition 4.4 implies that the image of the vector $\varphi_{\nu_{1}} \otimes \cdots \otimes \varphi_{\nu_{m}}$ in the quotient is not zero. Hence this image is highest relative to the action of the Yangian $Y\left(\mathfrak{g l}_{n}\right)$ on the quotient. Since the vectors $\varphi_{\nu_{1}}, \ldots, \varphi_{\nu_{m}}$ are highest in their $\mathrm{Y}\left(\mathfrak{g l}_{n}\right)$-modules, their tensor product is an eigenvector for all coefficients of the series $T_{i i}(x)$; again see (4.10). Moreover,

$$
T_{i i}(x)\left(\varphi_{\nu_{1}} \otimes \cdots \otimes \varphi_{\nu_{m}}\right)=\left(T_{i i}(x) \varphi_{\nu_{1}}\right) \otimes \cdots \otimes\left(T_{i i}(x) \varphi_{\nu_{m}}\right) .
$$

Therefore for any index $i=1, \ldots, n-1$, the Drinfeld polynomial $P_{i}(x)$ of the quotient is equal to the product of the Drinfeld polynomials with the same index $i$ of the $m$ tensor factors of (4.46). Thus we get the following theorem. Recall that here $\rho_{a}=m / 2-a+\frac{1}{2}$.

Theorem 5.1. Let the labels $\lambda_{1}, \ldots, \lambda_{m}$ satisfy the condition (5.1), while the labels

$$
\nu_{1}=\lambda_{1}-\mu_{1}, \ldots, \nu_{m}=\lambda_{m}-\mu_{m}
$$

belong to the set $\{0,1, \ldots, n\}$ and satisfy the condition (5.2). Then the quotient by the kernel of our intertwining operator (4.49) is a nonzero irreducible $\mathrm{Y}\left(\mathfrak{g l}_{n}\right)$ module. For any $i=1, \ldots, n-1$ the Drinfeld polynomial $P_{i}(x)$ of this module is the product of the differences $x-\mu_{a}-\rho_{a}$ taken over all indices a such that $\nu_{a}=i$. 
Note that if $\nu_{1}=\cdots=\nu_{m}=0$, then both the source and the target $\mathrm{Y}\left(\mathfrak{g l}_{n}\right)$ modules in (4.49) are trivial for any $\mu$. If moreover $\lambda+\rho$ is dominant, then by Proposition 4.4 our operator (4.49) is the identity map $\mathbb{C} \rightarrow \mathbb{C}$.

Now take any sequence of $n-1$ monic polynomials $P_{1}(x), \ldots, P_{n-1}(x)$ with complex coefficients. Denote by $\mathcal{P}$ the collection of pairs $(i, z)$ where $i=1, \ldots, n-1$ and $z$ ranges over all roots of the polynomial $P_{i}(x)$; the roots are taken with their multiplicities. Note that the collection $\mathcal{P}$ is unordered. Let $m$ be the total number of elements in $\mathcal{P}$; it is equal to the sum of the degrees of $P_{1}(x), \ldots, P_{n-1}(x)$. Suppose that at least one of the polynomials is not trivial, so that $m>0$. Let $\lambda$ and $\mu$ be any weights of $\mathfrak{g l}_{m}$ such that for $\nu=\lambda-\mu$ the collection of pairs $\left(\nu_{a}, \mu_{a}+\rho_{a}\right)$ for $a=1, \ldots, m$ coincides with $\mathcal{P}$. In particular $\nu_{a} \in\{1, \ldots, n-1\}$. We do not yet impose any other conditions on $\lambda$ and $\mu$.

Then the weights $\lambda$ and $\mu$ are determined up to any permutation of the $m$ pairs $\left(\nu_{a}, \mu_{a}+\rho_{a}\right)$. Equivalently, they are determined up to a permutation of the $m$ pairs $\left(\lambda_{a}+\rho_{a}, \mu_{a}+\rho_{a}\right)$. In other words, $\lambda$ and $\mu$ are determined up to the (simultaneous) shifted action of the Weyl group $\mathfrak{S}$ of $\mathfrak{g l}_{m}$ on them, as on elements of $\mathfrak{t}^{*}$. Therefore we can choose $\lambda$ to satisfy the conditions (5.1), so that the weight $\lambda+\rho$ is dominant. For $a<b$ the equality on the right-hand side of (5.2) means that $\lambda$ is invariant under the shifted action of that element of $\mathfrak{S}$ which exchanges $\eta_{a}$ with $\eta_{b}$, and leaves all other basis vectors of $\mathfrak{t}^{*}$ fixed. This action amounts to exchanging the pair $\left(\nu_{a}, \mu_{a}+\rho_{a}\right)$ with $\left(\nu_{b}, \mu_{b}+\rho_{b}\right)$. By using this action and keeping $\lambda$ fixed, we can choose $\mu$ so that the condition (5.2) is satisfied.

Now let us consider our intertwining operator (4.49) corresponding to the chosen weights $\lambda$ and $\mu$. Due to Theorem 5.1, the quotient by the kernel of this operator is a nonzero irreducible $\mathrm{Y}\left(\mathfrak{g l}_{n}\right)$-module, and has the given Drinfeld polynomials $P_{1}(x), \ldots, P_{n-1}(x)$. Up to equivalence and similarity, every nonzero irreducible finite-dimensional $\mathrm{Y}\left(\mathfrak{g l}_{n}\right)$-module thus arises as such a quotient. Note that the choice $\theta=-1$ here is essential.

5.2. Representations of twisted Yangians. From now on we will regard the coefficients of all the series $S_{i j}(x)$ as generators of the algebra $\mathrm{Y}\left(\mathfrak{g}^{\prime}\right)$ with the relations (4.2) and (4.18) imposed on them. In this subsection we collect some general results on finite-dimensional $\mathrm{Y}\left(\mathfrak{g}^{\prime}\right)$-modules for both $\mathfrak{g}^{\prime}=\mathfrak{s p}_{n}$ and $\mathfrak{g}^{\prime}=\mathfrak{s o}_{n}$. Let $\Psi$ be any of these modules. If $\Psi$ is obtained by restricting the trivial $Y\left(\mathfrak{g l}_{n}\right)$ module to the subalgebra $\mathrm{Y}\left(\mathfrak{g}^{\prime}\right) \subset \mathrm{Y}\left(\mathfrak{g l}_{n}\right)$, then $\Psi$ will also be called trivial.

Now assume that $\Psi$ is nonzero. We will use the ordering (4.56) of the indices $1, \ldots, n$. We will keep writing $i \prec j$ when $i$ precedes $j$ in the sequence (4.56). A nonzero vector of $\Psi$ is called highest if it is annihilated by all the coefficients of the series $S_{i j}(x)$ with $i \prec j$. If $\Psi$ is irreducible, then a highest vector $\psi \in \Psi$ is unique up to a scalar multiplier. Then $\psi$ is an eigenvector for the coefficients of all series $S_{i i}(x)$; see [M2, Theorem 4.2.6 and Corollary 4.2.7]. Then $\Psi$ is determined by the corresponding eigenvalues up to equivalence. In the next subsections we will use the description from [M2, Chapter 4] of all possible eigenvalues. Note that the relation (4.18) with $i=j$ takes the form

$$
S_{i i}(x) \mp 2 x S_{\tilde{\imath} \tilde{\imath}}(x)=(1 \mp 2 x) S_{i i}(-x) .
$$

Hence it suffices to describe the eigenvalues of the coefficients of only one of every two series $S_{i i}(x)$ and $S_{\tilde{\imath} \tilde{\imath}}(x)$. We choose the series $S_{i i}(x)$ with $i \succcurlyeq \tilde{\imath}$, or equivalently with $i \succcurlyeq n$. This choice is explained by the next lemma which is valid for any 
nonzero $\mathrm{Y}\left(\mathfrak{g l}_{n}\right)$-module $\Phi$, not necessarily irreducible; cf. [M2, Corollary 4.2.10]. We will also regard $\Phi$ as module over the subalgebra $\mathrm{Y}\left(\mathfrak{g}^{\prime}\right) \subset \mathrm{Y}\left(\mathfrak{g l}_{n}\right)$ by restriction.

Lemma 5.2. Let $\varphi \in \Phi$ be a nonzero vector annihilated by all coefficients of the series $T_{i j}(x)$ with $i \prec j$. Suppose $T_{i i}(x) \varphi=h_{i}(x) \varphi$ for $i=1, \ldots, n$ where $h_{i}(x)$ is a formal power series in $x^{-1}$ with coefficients from $\mathbb{C}$. Then $\varphi$ is a highest vector for $\mathrm{Y}\left(\mathfrak{g}^{\prime}\right)$ and moreover $S_{i i}(x) \varphi=g_{i}(x) \varphi$ for $i=1, \ldots, n$ where

$$
g_{i}(x)=\left\{\begin{array}{cl}
\left(1 \mp(2 x)^{-1}\right) h_{i}(x) h_{\tilde{\imath}}(-x) \pm(2 x)^{-1} h_{i}(-x) h_{\tilde{\imath}}(x) & \text { if } \quad i \prec \tilde{\imath} ; \\
h_{i}(x) h_{\tilde{\imath}}(-x) & \text { if } \quad i \succcurlyeq \tilde{\imath} .
\end{array}\right.
$$

Proof. Take $i, j \in\{1, \ldots, n\}$ such that $i \preccurlyeq j$. The coefficients of the series $S_{i j}(x)$ act on the vector $\varphi \in \Phi$ as the corresponding coefficients of the series (4.13), giving the sum of

$$
\theta_{i} \theta_{k} T_{\tilde{k} \tilde{\imath}}(-x) T_{k j}(x) \varphi
$$

over the indices $k \succcurlyeq j$. This is because the vector $\varphi$ is highest relative to $\mathrm{Y}\left(\mathfrak{g l}_{n}\right)$. Using the commutation relations (4.5), the summand (5.3) is equal to the difference

$$
\begin{gathered}
\theta_{i} \theta_{k} T_{k j}(x) T_{\tilde{k} \tilde{\imath}}(-x) \varphi \\
-\theta_{i} \theta_{k}(2 x)^{-1}\left(T_{k \tilde{\imath}}(-x) T_{\tilde{k} j}(x)-T_{k \tilde{\imath}}(-x) T_{\tilde{k} j}(x)\right) \varphi .
\end{gathered}
$$

Here (5.4) may be nonzero only if $\tilde{k} \succcurlyeq \tilde{\imath}$, that is, only if $k \preccurlyeq i$. The latter condition together with $i \preccurlyeq j$ and $j \preccurlyeq k$ implies that $i=j=k$. Then (5.4) equals

$$
h_{i}(x) h_{\tilde{\imath}}(-x) \varphi \text {. }
$$

Further, (5.5) may be nonzero only if $\tilde{k} \succcurlyeq j$. When presenting (5.3) as the sum of (5.4) and of (5.5) by using (4.5), we could replace (5.5) by the expression

$$
-\theta_{i} \theta_{k}(2 x)^{-1}\left(T_{\tilde{k} j}(x) T_{k \tilde{\imath}}(-x)-T_{\tilde{k} j}(x) T_{k \tilde{\imath}}(-x)\right) \varphi .
$$

But (5.7) may be nonzero only if $k \succcurlyeq \tilde{\imath}$, that is, only if $\tilde{k} \preccurlyeq i$. The latter condition together with $i \preccurlyeq j$ and $j \preccurlyeq \tilde{k}$ implies that $i=j=\tilde{k}$. Then (5.5) and (5.7) are equal to

$$
\mp(2 x)^{-1}\left(h_{i}(x) h_{\tilde{\imath}}(-x)-h_{i}(-x) h_{\tilde{\imath}}(x)\right) \varphi .
$$

We have also assumed that $k \succcurlyeq j$, which together with the equalities $i=j=\tilde{k}$ implies that $i \preccurlyeq \tilde{\imath}$. Moreover, if $i=\tilde{\imath}$, then (5.8) obviously vanishes.

Thus under the assumption $i \preccurlyeq j$, we have proved that $S_{i j}(x) \varphi$ may difer from zero only of $i=j$. If $i \prec \tilde{\imath}$, then $S_{i i}(x) \varphi$ is equal to the sum of (5.6) and (5.8). But if $i \succcurlyeq \tilde{\imath}$, then $S_{i i}(x) \varphi$ is equal to the expression (5.6) alone.

We will keep assuming that $\theta=-1$. So for $\mathfrak{g}=\mathfrak{s p}_{2 m}$ or $\mathfrak{g}=\mathfrak{s o}_{2 m}$ we will have $\mathfrak{g}^{\prime}=\mathfrak{s p}_{n}$ or $\mathfrak{g}^{\prime}=\mathfrak{s o}_{n}$, respectively. In the next subsections, we consider the two cases separately. Moreover, we will separate the cases of $\mathfrak{g}^{\prime}=\mathfrak{s o}_{n}$ with $n$ even and odd. If $\mathfrak{g}^{\prime}=\mathfrak{s p}_{n}$, then $n$ is even. We will write $n=2 l$ if $n$ is even, or $n=2 l+1$ if $n$ is odd. Then the condition $i \succcurlyeq n$ will mean, respectively, that $i$ is one of the indices

$$
2 l, 2 l-2, \ldots, 2 \quad \text { or } \quad 2 l+1,2 l, 2 l-2, \ldots, 2 .
$$

As the first application of Lemma 5.2, consider the case when $\Phi=\Phi_{t}^{k}$ and $\varphi=\psi_{k}$ is the vector of $\Phi^{k}$ defined in Subsection 4.4. We assume that $k \in\{0,1, \ldots, n\}$. All the conditions of Lemma 5.2 are then satisfied. Here $h_{i}(x)=1+(x-t)^{-1}$ if 
$i$ is one of the first $k$-indices in the sequence (4.56); otherwise $h_{i}(x)=1$. Hence $h_{\tilde{\imath}}(-x)=1-(x+t)^{-1}$ if $i$ is one of the last $k$-indices in the sequence (4.56); otherwise $h_{\tilde{\imath}}(-x)=1$. If $i \succcurlyeq n$, then $g_{i}(x)=h_{i}(x) h_{\tilde{\imath}}(-x)$. This implies the following relations, to be used later on.

Take any $i \succ n$. Let $j$ be the index occuring just before $i$ in the sequence (4.56). Here $j=i+2$ unless $n=2 l+1$ and $i=2 l$, in which case $j=i+1$. Note that $j \succcurlyeq n$. Then

$$
g_{j}(x) g_{i}(x)^{-1}=\left\{\begin{array}{cl}
(x+t)(x+t-1)^{-1} & \text { if } 2 k=i ; \\
(x-t+1)(x-t)^{-1} & \text { if } 2(n-k)=i ; \\
1 & \text { otherwise. }
\end{array}\right.
$$

Indeed, for $i \succ n$, we have $h_{i}(x) \neq h_{j}(x)$ only if $2(n-k)=i$. In the latter case we have $h_{i}(x)=1$ while $h_{j}(x)=1+(x-t)^{-1}$. Further, for any $i \succ n$, we have $h_{\tilde{\imath}}(x) \neq h_{\tilde{\jmath}}(x)$ only if $2 k=i$. In the latter case we have $h_{\tilde{\imath}}(x)=1+(x-t)^{-1}$ while $h_{\tilde{\jmath}}(x)=1$. Thus we obtain the relation displayed above.

If $n=2 l$, then we also have the relation

$$
g_{n}(-x) g_{n}(x)^{-1}=\left\{\begin{array}{cl}
(x-t+1)(x+t)(x-t)^{-1}(x+t-1)^{-1} & \text { if } k=l ; \\
1 & \text { otherwise }
\end{array}\right.
$$

Indeed, if $n=2 l$, then $\tilde{n}=2 l-1$. Then for $k \leqslant l$, we have $h_{n}(x)=1$, while for $k>l$, we have $h_{n}(x)=1+(x-t)^{-1}$. Further, for $k<l$, we have $h_{\tilde{n}}(x)=1$, while for $k \geqslant l$, we have $h_{\tilde{n}}(x)=1+(x-t)^{-1}$. Therefore $g_{n}(x)=g_{n}(-x)$ for any $k \neq l$. But if $k=l$, then $g_{n}(x)=1+(x-t)^{-1}$. Thus we obtain the last displayed relation.

We end this section by introducing a notion applicable to any finite-dimensional module $\Psi$ over the twisted Yangian $\mathrm{Y}\left(\mathfrak{g}^{\prime}\right)$ where $\mathfrak{g}^{\prime}=\mathfrak{s o}_{n}$ and $n$ is any positive integer, even or odd. Consider the restriction of $\Psi$ to the subalgebra $\mathrm{U}\left(\mathfrak{s o}_{n}\right)$ of $\mathrm{Y}\left(\mathfrak{s o}_{n}\right)$. Suppose that the restriction integrates to a module of the complex special orthogonal group $\mathrm{SO}_{n}$. Thus we exclude the spinor representations of $\mathfrak{s o}_{n}$. Then we call the $\mathrm{Y}\left(\mathfrak{s o}_{n}\right)$-module $\Psi$ integrable. Equivalently, then $\Psi$ is an $\left(\mathrm{Y}\left(\mathfrak{s o}_{n}\right), \mathrm{SO}_{n}\right)$ module; see the beginning of Subsection 4.4. For instance, any $\mathrm{Y}\left(\mathfrak{s o}_{n}\right)$-module of the form (4.51) is integrable, and so is any quotient of such a module. We will not treat nonintegrable $\mathrm{Y}\left(\mathfrak{s o}_{n}\right)$-modules here.

5.3. Irreducible representations of $\mathrm{Y}\left(\mathfrak{s p}_{n}\right)$. In this subsection, we consider the case when $\theta=-1$ and $\mathfrak{g}=\mathfrak{s p}_{2 m}$, so that $\mathfrak{g}^{\prime}=\mathfrak{s p}_{n}$ where $n=2 l$. Let $\Psi$ be a nonzero irreducible finite-dimensional $\mathrm{Y}\left(\mathfrak{s p}_{n}\right)$-module. Let $\psi \in \Psi$ be a highest vector. By [M2, Theorem 4.3.8] for any $k=1, \ldots, l-1$, we have

$$
S_{2 k+2,2 k+2}(x) S_{2 k, 2 k}(x)^{-1} \psi=Q_{k}\left(x+\frac{1}{2}\right) Q_{k}\left(x-\frac{1}{2}\right)^{-1} \psi,
$$

where $Q_{k}(x)$ is a monic polynomial in $x$ with coefficients in $\mathbb{C}$. Further, we have

$$
S_{n n}(-x) S_{n n}(x)^{-1} \psi=Q_{l}\left(x+\frac{1}{2}\right) Q_{l}\left(x-\frac{1}{2}\right)^{-1} \psi,
$$

where $Q_{l}(x)$ is an even monic polynomial in $x$ with coefficients in the field $\mathbb{C}$. Any sequence of $l$ monic polynomials with complex coefficients arises in this way, provided the last polynomial in the sequence is even. Further, two irreducible finite-dimensional $\mathrm{Y}\left(\mathfrak{s p}_{n}\right)$-modules have the same polynomials $Q_{1}(x), \ldots, Q_{l}(x)$ if and only if their restrictions to the subalgebra $\mathrm{SY}\left(\mathfrak{s p}_{n}\right) \subset \mathrm{Y}\left(\mathfrak{s p}_{n}\right)$ are equivalent. Thus the nonzero irreducible finite-dimensional $\mathrm{Y}\left(\mathfrak{s p}_{n}\right)$-modules are parametrized by their sequences of polynomials $Q_{1}(x), \ldots, Q_{l}(x)$ up to equivalence and similarity 
[M2, Corollary 4.3.11]. For example, $Q_{1}(x)=\cdots=Q_{l}(x)=1$ for the trivial $\mathrm{Y}\left(\mathfrak{s p}_{n}\right)$-module $\Psi$.

In this subsection, $\rho_{a}=-a$ and $\kappa_{a}=-l$ for each index $a=1, \ldots, m$. Recall the definition (2.18) of the weight $\nu$. Suppose that each label $\nu_{a} \in\{0,1, \ldots, n\}$ and that the weight $\lambda+\rho$ is dominant. The latter condition here means that

$$
\begin{aligned}
\lambda_{b}-\lambda_{a}+\rho_{b}-\rho_{a} \neq 1,2, \ldots & \text { for all } 1 \leqslant a<b \leqslant m ; \\
\lambda_{a}+\lambda_{b}+\rho_{a}+\rho_{b} \neq 1,2, \ldots & \text { for all } 1 \leqslant a<b \leqslant m ; \\
\lambda_{a}+\rho_{a} \neq 1,2, \ldots & \text { for all } 1 \leqslant a \leqslant m .
\end{aligned}
$$

By Corollary 3.9 and Proposition 4.2, the quotient by the kernel of our intertwining operator (4.58) is then an irreducible $\mathrm{Y}\left(\mathfrak{s p}_{n}\right)$-module. But the definition (4.10) of the comultiplication on $\mathrm{Y}\left(\mathfrak{g l}_{n}\right)$ implies that the vector (4.57) is highest relative to the twisted Yangian $\mathrm{Y}\left(\mathfrak{s p}_{n}\right)$. Here we also use Lemma 5.2, Further suppose that

$$
\begin{array}{cllll}
\nu_{a} \geqslant \nu_{b} & \text { whenever } & \lambda_{a}-\lambda_{b}+\rho_{a}-\rho_{b}=0 \quad \text { and } & a<b ; \\
\nu_{a}+\nu_{b} \leqslant n & \text { whenever } & \lambda_{a}+\lambda_{b}+\rho_{a}+\rho_{b}=0 & \text { and } & a<b ; \\
\nu_{a} \leqslant l & \text { whenever } & \lambda_{a}+\rho_{a}=0 . & &
\end{array}
$$

Due to the dominance of the weight $\lambda+\rho$, Proposition 4.6 then implies that the image of the vector (4.57) in the quotient is not zero. Hence this image is highest relative to the action of the twisted Yangian $\mathrm{Y}\left(\mathfrak{s p}_{n}\right)$ on the quotient. Lemma 5.2 implies that each of the polynomials $Q_{1}(x), \ldots, Q_{l}(x)$ of the quotient is multiplicative with respect to the $m$ tensor factors of the vector (4.57). But in the case $m=1$, these polynomials are transparent from the relations given at the very end of Subsection 5.2. Thus we get

Theorem 5.3. Put $n=2 l$. Let $\lambda_{1}, \ldots, \lambda_{m}$ obey (5.9) (5.11) while the labels

$$
\nu_{1}=\lambda_{1}-\mu_{1}+l, \ldots, \nu_{m}=\lambda_{m}-\mu_{m}+l
$$

belong to the set $\{0,1, \ldots, n\}$ and satisfy (5.12) $-(5.14)$. Then the quotient by the kernel of our intertwining operator (4.58) is a nonzero irreducible $\mathrm{Y}\left(\mathfrak{s p}_{n}\right)$-module. For any $k=1, \ldots, l-1$, the polynomial $Q_{k}(x)$ of this module is the product of the sums $x+\mu_{a}+\rho_{a}$ taken over all indices a such that $\nu_{a}=k$, and of the differences $x-\mu_{a}-\rho_{a}$ taken over all indices a such that $\nu_{a}=n-k$. The polynomial $Q_{l}(x)$ of this module is the product of the differences $x^{2}-\left(\mu_{a}+\rho_{a}\right)^{2}$ taken over all indices a such that $\nu_{a}=l$.

Note that if $\nu_{1}=\cdots=\nu_{m}=0$, then both the source and the target $\mathrm{Y}\left(\mathfrak{s p}_{n}\right)$ modules in (4.58) are trivial for any $\mu$. If moreover $\lambda+\rho$ is dominant, then by Proposition 4.6 our operator 4.58) is the identity map $\mathbb{C} \rightarrow \mathbb{C}$.

Now let us take any sequence of $l$ monic polynomials $Q_{1}(x), \ldots, Q_{l}(x)$ with complex coefficients, such that the polynomial $Q_{l}(x)$ is even. Let $m$ be the sum of the degrees of $Q_{1}(x), \ldots, Q_{l-1}(x)$ plus half of the degree of $Q_{l}(x)$. Suppose that at least one of all the $l$ polynomials is not trivial, so that $m>0$. Let $\lambda$ and $\mu$ be any weights of $\mathfrak{s p}_{2 m}$ such that the corresponding labels (5.15) belong to the set $\{1, \ldots, n-1\}$ and such that the given polynomials $Q_{1}(x), \ldots, Q_{l}(x)$ are obtained from $\lambda$ and $\mu$ as in Theorem 5.3. But we do not yet impose any other conditions on $\lambda$ and $\mu$, such as dominance of $\lambda+\rho$.

Then $\lambda$ and $\mu$ are determined up to permuting the $m$ pairs $\left(\nu_{a}, \mu_{a}+\rho_{a}\right)$, and up to replacing $\left(\nu_{a}, \mu_{a}+\rho_{a}\right)$ by $\left(n-\nu_{a},-\mu_{a}-\rho_{a}\right)$ for any number of indices $a$. 
Equivalently, the weights $\lambda$ and $\mu$ are determined up to a permutation of the $m$ pairs $\left(\lambda_{a}+\rho_{a}, \mu_{a}+\rho_{a}\right)$, and up to replacing $\left(\lambda_{a}+\rho_{a}, \mu_{a}+\rho_{a}\right)$ by $\left(-\lambda_{a}-\rho_{a},-\mu_{a}-\rho_{a}\right)$ for any number of indices $a$. In other words, $\lambda$ and $\mu$ are determined up to the (simultaneous) shifted action of the Weyl group $\mathfrak{S}$ of $\mathfrak{s p}_{2 m}$ on them, as on elements of $\mathfrak{t}^{*}$. Therefore we can choose $\lambda$ to satisfy the conditions (5.9)-(5.11) so that the weight $\lambda+\rho$ is dominant.

The equality on the right-hand side of (5.12) for $a<b$ means that $\lambda$ is invariant under the shifted action of that element of $\mathfrak{S}$ which exchanges $\eta_{m-a+1}$ with $\eta_{m-b+1}$, and leaves all other basis vectors of $t^{*}$ fixed. This action amounts to exchanging the pair $\left(\nu_{a}, \mu_{a}+\rho_{a}\right)$ with $\left(\nu_{b}, \mu_{b}+\rho_{b}\right)$. By using this action and keeping $\lambda$ fixed, we can choose $\mu$ so that the condition (5.12) is satisfied.

The equality on the right-hand side of (5.14) means that the weight $\lambda$ is invariant under the shifted action of that element of $\mathfrak{S}$ which maps $\eta_{m-a+1}$ to $-\eta_{m-a+1}$, and leaves all other basis vectors of $\boldsymbol{t}^{*}$ fixed. This action amounts to replacing the pair $\left(\nu_{a}, \mu_{a}+\rho_{a}\right)$ by the pair $\left(n-\nu_{a},-\mu_{a}-\rho_{a}\right)$. By using this action and keeping $\lambda$ fixed, we can choose $\mu$ so that the condition (5.14) is satisfied.

Finally, the equality on the right-hand side of (5.13) for $a<b$ means that the weight $\lambda$ is invariant under the shifted action of that element of the group $\mathfrak{S}$ which maps $\eta_{m-a+1}$ and $\eta_{m-b+1}$ to $-\eta_{m-b+1}$ and $-\eta_{m-a+1}$, respectively, leaving all other basis vectors of $\mathfrak{t}^{*}$ fixed. This action amounts to replacing the pairs $\left(\nu_{a}, \mu_{a}+\rho_{a}\right)$ and $\left(\nu_{b}, \mu_{b}+\rho_{b}\right)$ by the pairs $\left(n-\nu_{b},-\mu_{b}-\rho_{b}\right)$ and $\left(n-\nu_{a},-\mu_{a}-\rho_{a}\right)$, respectively. By using this action and keeping $\lambda$ fixed, we can choose the weight $\mu$ so that the condition (5.13) is satisfied when $\lambda_{a}+\rho_{a} \neq 0$, or equivalently when $\lambda_{b}+\rho_{b} \neq 0$. When $\lambda_{a}+\rho_{a}=0$ and $\lambda_{b}+\rho_{b}=0$, the condition (5.13) is already satisfied because then $\nu_{a} \leqslant l$ and $\eta_{b} \leqslant l$ due to (5.14).

Now let us consider our intertwining operator (4.58) corresponding to the weights $\lambda$ and $\mu$. Due to Theorem 5.3 the quotient by the kernel of this operator is a nonzero irreducible $\mathrm{Y}\left(\mathfrak{s p}_{n}\right)$-module, and has the given polynomials $Q_{1}(x), \ldots, Q_{l}(x)$. Thus up to equivalence and similarity, each nonzero irreducible finite-dimensional $\mathrm{Y}\left(\mathfrak{s p}_{n}\right)$-module arises as such a quotient. The choice $\theta=-1$ here was essential.

5.4. Irreducible representations of $\mathrm{Y}\left(\mathfrak{s o}_{n}\right)$ for odd $n$. In this subsection, we consider the case of $\mathfrak{g}^{\prime}=\mathfrak{s o}_{n}$ where $n$ is odd. Hence $n=2 l+1$ where $l$ is a nonnegative integer. We assume that $\theta=-1$, so that $\mathfrak{g}=\mathfrak{s o}_{2 m}$. Let $\Psi$ be any integrable finite-dimensional $\mathrm{Y}\left(\mathfrak{s o}_{n}\right)$-module; see the end of Subsection 5.2. Further suppose that the $\mathrm{Y}\left(\mathfrak{s o}_{n}\right)$-module $\Psi$ is irreducible and nonzero. Let $\psi \in$ $\Psi$ be a highest vector. By [M2, Theorem 4.5.9] there exist monic polynomials $Q_{1}(x), \ldots, Q_{l}(x)$ in $x$ with complex coefficients such that for any $k=1, \ldots, l-1$

$$
S_{2 k+2,2 k+2}(x) S_{2 k, 2 k}(x)^{-1} \psi=Q_{k}\left(x+\frac{1}{2}\right) Q_{k}\left(x-\frac{1}{2}\right)^{-1} \psi,
$$

while

$$
S_{n n}(x) S_{n-1, n-1}(x)^{-1} \psi=Q_{l}\left(x+\frac{1}{2}\right) Q_{l}\left(x-\frac{1}{2}\right)^{-1} \psi .
$$

Every sequence of $l$ monic polynomials with coefficients from $\mathbb{C}$ arises in this way. Two irreducible integrable finite-dimensional $\mathrm{Y}\left(\mathfrak{s o}_{n}\right)$-modules have the same polynomials $Q_{1}(x), \ldots, Q_{l}(x)$ if and only if their restrictions to the subalgebra $\mathrm{SY}\left(\mathfrak{s o}_{n}\right) \subset \mathrm{Y}\left(\mathfrak{s o}_{n}\right)$ are equivalent $\mathrm{M2}$, Corollary 4.5.12]. Thus all the nonzero irreducible integrable finite-dimensional $\mathrm{Y}\left(\mathfrak{s o}_{n}\right)$-modules are parametrized by their polynomials $Q_{1}(x), \ldots, Q_{l}(x)$ up to equivalence and similarity. For example, if $\Psi$ is the trivial $\mathrm{Y}\left(\mathfrak{s o}_{n}\right)$-module, then we have $Q_{1}(x)=\cdots=Q_{l}(x)=1$. 
In this subsection, $\rho_{a}=1-a$ and $\kappa_{a}=-l+\frac{1}{2}$ for each index $a=1, \ldots, m$. Recall the definition (2.18) of the weight $\nu$. Suppose that each label $\nu_{a} \in\{0,1, \ldots, n\}$ and that the weight $\lambda+\rho$ is dominant. Here the latter condition means that

$$
\begin{array}{ll}
\lambda_{b}-\lambda_{a}+\rho_{b}-\rho_{a} \neq 1,2, \ldots & \text { for all } 1 \leqslant a<b \leqslant m ; \\
\lambda_{a}+\lambda_{b}+\rho_{a}+\rho_{b} \neq 1,2, \ldots & \text { for all } 1 \leqslant a<b \leqslant m .
\end{array}
$$

At the end of Subsection 4.4 we observed that for $\mathfrak{g}=\mathfrak{s o}_{2 m}$ our operators (4.58) and (4.59) are the same. Using Proposition 4.2 and Corollary 4.9, the quotient by the kernel of this operator is an irreducible $\mathrm{Y}\left(\mathfrak{s o}_{n}\right)$-module. But the definition (4.10) of the comultiplication on $\mathrm{Y}\left(\mathfrak{g l}_{n}\right)$ implies that the vector (4.57) is highest relative to the twisted Yangian $\mathrm{Y}\left(\mathfrak{s o}_{n}\right)$. Here we also use Lemma 5.2. Further suppose that

$$
\begin{aligned}
& \nu_{a} \geqslant \nu_{b} \quad \text { whenever } \quad \lambda_{a}-\lambda_{b}+\rho_{a}-\rho_{b}=0 \text { and } a<b \text {; } \\
& \nu_{a}+\nu_{b} \leqslant n \quad \text { whenever } \lambda_{a}+\lambda_{b}+\rho_{a}+\rho_{b}=0 \text { and } a<b \text {. }
\end{aligned}
$$

We have already noted that for $\sigma=\sigma_{0}$ and $\omega=\tau_{m} \sigma_{0}$ our operator (4.53) is invertible. Due to the dominance of the weight $\lambda+\rho$, Proposition 4.6 now implies that the image of the vector (4.57) in the quotient is not zero. Hence this image is highest relative to the action of the twisted Yangian $\mathrm{Y}\left(\mathfrak{s o}_{n}\right)$ on the quotient. Lemma 5.2 implies that each of the polynomials $Q_{1}(x), \ldots, Q_{l}(x)$ of the quotient is multiplicative with respect to the $m$ tensor factors of the vector (4.57). But in the case $m=1$ these polynomials are transparent from the relations given at the very end of Subsection 5.2. Thus we get

Theorem 5.4. Put $n=2 l+1$. Let $\lambda_{1}, \ldots, \lambda_{m}$ satisfy (5.16), (5.17) while the labels

$$
\nu_{1}=\lambda_{1}-\mu_{1}+l+\frac{1}{2}, \ldots, \nu_{m}=\lambda_{m}-\mu_{m}+l+\frac{1}{2}
$$

belong to the set $\{0,1, \ldots, n\}$ and satisfy (5.18), (5.19). Then the quotient by the kernel of our intertwining operator (4.58) is a nonzero irreducible $\mathrm{Y}\left(\mathfrak{s o}_{n}\right)$-module. For any $k=1, \ldots, l$ the polynomial $Q_{k}(x)$ of this module is the product of the sums $x+\mu_{a}+\rho_{a}$ taken over all indices a such that $\nu_{a}=k$, and of the differences $x-\mu_{a}-\rho_{a}$ taken over all indices a such that $\nu_{a}=n-k$.

Note that if $\nu_{1}=\cdots=\nu_{m}=0$, then both the source and the target $\mathrm{Y}\left(\mathfrak{s o}_{n}\right)$ modules in (4.58) are trivial for any $\mu$. If moreover $\lambda+\rho$ is dominant, then by Proposition 4.6 our operator (4.58) is the identity map $\mathbb{C} \rightarrow \mathbb{C}$.

Now let us take any sequence of $l$ monic polynomials $Q_{1}(x), \ldots, Q_{l}(x)$ with complex coefficients. Let $m$ be the sum of the degrees of $Q_{1}(x), \ldots, Q_{l}(x)$. Suppose that $m>0$. Let $\lambda$ and $\mu$ be any weights of $\mathfrak{s o}_{2 m}$ such that the corresponding labels (5.20) belong to the set $\{1, \ldots, n-1\}$ and such that the given polynomials $Q_{1}(x), \ldots, Q_{l}(x)$ are obtained from $\lambda$ and $\mu$ as in Theorem 5.4. We do not yet impose any other conditions on $\lambda$ and $\mu$.

Then $\lambda$ and $\mu$ are determined up to permuting the $m$ pairs $\left(\nu_{a}, \mu_{a}+\rho_{a}\right)$ and up to replacing $\left(\nu_{a}, \mu_{a}+\rho_{a}\right)$ by $\left(n-\nu_{a},-\mu_{a}-\rho_{a}\right)$ for any number of indices $a$. Equivalently, the weights $\lambda$ and $\mu$ are determined up to a permutation of the $m$ pairs $\left(\lambda_{a}+\rho_{a}, \mu_{a}+\rho_{a}\right)$, and up to replacing $\left(\lambda_{a}+\rho_{a}, \mu_{a}+\rho_{a}\right)$ by $\left(-\lambda_{a}-\rho_{a},-\mu_{a}-\rho_{a}\right)$ for any number of indices $a$. Thus $\lambda$ and $\mu$ are determined up to the (simultaneous) shifted action of the group $\mathfrak{R}$ of $\mathfrak{s o}_{2 m}$ on them, as on elements of $\mathfrak{t}^{*}$. Using only the action of the subgroup $\mathfrak{S} \subset \mathfrak{R}$, we can choose $\lambda$ to satisfy the conditions (5.16), (5.17) so that the weight $\lambda+\rho$ is dominant. 
The equality on the right-hand side of (5.18) for $a<b$ means that $\lambda$ is invariant under the shifted action of that element of $\mathfrak{S}$ which exchanges $\eta_{m-a+1}$ with $\eta_{m-b+1}$, and leaves all other basis vectors of $\mathfrak{t}^{*}$ fixed. This action amounts to exchanging the pair $\left(\nu_{a}, \mu_{a}+\rho_{a}\right)$ with $\left(\nu_{b}, \mu_{b}+\rho_{b}\right)$. By using this action and keeping $\lambda$ fixed, we can choose $\mu$ so that the condition (5.18) is satisfied.

The equality on the right-hand side of (5.19) for $a<b$ means that the weight $\lambda$ is invariant under the shifted action of that element of the group $\mathfrak{S}$ which maps $\eta_{m-a+1}$ and $\eta_{m-b+1}$ to $-\eta_{m-b+1}$ and $-\eta_{m-a+1}$, respectively, leaving all other basis vectors of $\mathfrak{t}^{*}$ fixed. This action amounts to replacing the pairs $\left(\nu_{a}, \mu_{a}+\rho_{a}\right)$ and $\left(\nu_{b}, \mu_{b}+\rho_{b}\right)$ by the pairs $\left(n-\nu_{b},-\mu_{b}-\rho_{b}\right)$ and $\left(n-\nu_{a},-\mu_{a}-\rho_{a}\right)$, respectively. By using this action and keeping $\lambda$ fixed, we can choose the weight $\mu$ so that the condition (5.19) is satisfied.

Now let us consider our intertwining operator (4.58) corresponding to these weights $\lambda$ and $\mu$. Due to Theorem 5.4, the quotient by the kernel of this operator is a nonzero irreducible integrable $\mathrm{Y}\left(\mathfrak{s o}_{n}\right)$-module, and has the given polynomials $Q_{1}(x), \ldots, Q_{l}(x)$. Thus up to equivalence and similarity, every nonzero irreducible integrable finite-dimensional $\mathrm{Y}\left(\mathfrak{s o}_{n}\right)$-module arises as such a quotient. Note that the choice $\theta=-1$ here is essential.

5.5. Irreducible representations of $\mathrm{Y}\left(\mathfrak{s o}_{n}\right)$ for even $n$. In this subsection, we will consider the case of $\mathfrak{g}^{\prime}=\mathfrak{s o}_{n}$ where $n$ is even. Hence $n=2 l$ where $l$ is a positive integer. We keep assuming that $\theta=-1$, so that $\mathfrak{g}=\mathfrak{s o}_{2 m}$. Let $\Psi$ be any $\mathrm{Y}\left(\mathfrak{s o}_{n}\right)$-module. Let $v \in \mathrm{O}_{n}$ be the element which exchanges the basis vector $f_{n-1}$ with $f_{n}$, and leaves all other basis vectors of $\mathbb{C}^{n}$ fixed. Consider the corresponding automorphism (4.52) of the algebra $\mathrm{Y}\left(\mathfrak{s o}_{n}\right)$. Our element $v$ is involutive, and so is the corresponding automorphism of $\mathrm{Y}\left(\mathfrak{s o}_{n}\right)$. Denote by $\bar{\Psi}$ the $\mathrm{Y}\left(\mathfrak{s o}_{n}\right)$-module obtained by pulling the action of $\mathrm{Y}\left(\mathfrak{s o}_{n}\right)$ on $\Psi$ back through this automorphism. Any $\mathrm{Y}\left(\mathfrak{s o}_{n}\right)$-module equivalent to $\bar{\Psi}$ will be called conjugate to $\Psi$.

The $\mathrm{Y}\left(\mathfrak{s o}_{n}\right)$-modules $\Psi$ and $\bar{\Psi}$ may be equivalent or not. Suppose that the $\mathrm{Y}\left(\mathfrak{s o}_{n}\right)$-module $\Psi$ is irreducible, finite dimensional, integrable and nonzero. Then the $\mathrm{Y}\left(\mathfrak{s o}_{n}\right)$-module $\bar{\Psi}$ is also. By [M2, Theorem 4.4.14] for a highest vector $\psi$ of at least one of the two modules $\Psi$ and $\bar{\Psi}$, we have for $k=1, \ldots, l-1$ that

$$
S_{2 k+2,2 k+2}(x) S_{2 k, 2 k}(x)^{-1} \psi=Q_{k}\left(x+\frac{1}{2}\right) Q_{k}\left(x-\frac{1}{2}\right)^{-1} \psi,
$$

where $Q_{k}(x)$ is a monic polynomial in $x$ with coefficients in $\mathbb{C}$. Further, we have

$$
S_{n n}(-x) S_{n n}(x)^{-1} \psi=Q_{l}\left(x+\frac{1}{2}\right) Q_{l}\left(x-\frac{1}{2}\right)^{-1} \psi,
$$

where $Q_{l}(x)$ is an even monic polynomial in $x$ with coefficients in $\mathbb{C}$. Any sequence of $l$ monic polynomials with complex coefficients arises in this way, provided that the last polynomial in the sequence is even. Moreover, the two $\mathrm{Y}\left(\mathfrak{s o}_{n}\right)$-modules $\Psi$ and $\bar{\Psi}$ are equivalent, if and only if zero is not a root of the corresponding polynomial $Q_{l}(x)$.

Let us consider the case when $\Psi$ and $\bar{\Psi}$ are not equivalent, so that zero is a root of the polynomial $Q_{l}(x)$. Let $h$ be the positive integer such that $0,1, \ldots, h-1$ are roots of $Q_{l}(x)$, but $h$ is not. The above vector $\psi$ has been a highest vector of one of the two $\mathrm{Y}\left(\mathfrak{s o}_{n}\right)$-modules $\Psi$ and $\bar{\Psi}$. Let $\bar{\psi}$ be a highest vector of the other of the two. For each index $i \succcurlyeq n$ we have $S_{i i}(x) \psi=g_{i}(x) \psi$, where $g_{i}(x)$ is a formal power series in $x^{-1}$ with the coefficients from $\mathbb{C}$. The proof of [M2, Theorem 4.4.14] demonstrates that then for each $i \succ n$ we also have the equality $S_{i i}(x) \bar{\psi}=g_{i}(x) \bar{\psi}$, 
while

$$
S_{n n}(x) \bar{\psi}=g(x) g_{n}(x) \bar{\psi}
$$

where

$$
g(x)=\left(x+h+\frac{1}{2}\right)\left(x-h+\frac{1}{2}\right)^{-1} .
$$

By the definition of the polynomial $Q_{l}(x)$ here we have

$$
g_{n}(-x) g_{n}(x)^{-1}=Q_{l}\left(x+\frac{1}{2}\right) Q_{l}\left(x-\frac{1}{2}\right)^{-1} .
$$

However,

$$
g(-x) g(x)^{-1} \neq Q\left(x+\frac{1}{2}\right) Q\left(x-\frac{1}{2}\right)^{-1}
$$

for any polynomial $Q(x)$, because the integer $h$ is positive. This implies that only one of the two nonequivalent $\mathrm{Y}\left(\mathfrak{s o}_{n}\right)$-modules $\Psi$ and $\bar{\Psi}$ gives rise to $l$ polynomials by using its highest vector $\psi$, as in (5.21) and (5.22). If $\psi$ is a highest vector of $\Psi$, assign to $\Psi$ a label $\delta=1$. Otherwise, that is, if $\psi$ is a highest vector of $\bar{\Psi}$, assign to $\Psi$ a label $\delta=-1$. The polynomials $Q_{1}(x), \ldots, Q_{l}(x)$ will be associated to both $\Psi$ and $\bar{\Psi}$ from now on.

Thus to any nonzero irreducible integrable finite-dimensional $\mathrm{Y}\left(\mathfrak{s o}_{n}\right)$-module $\Psi$ we have associated a sequence of monic polynomials $Q_{1}(x), \ldots, Q_{l}(x)$, where the last polynomial is even. If zero is a root of the polynomial $Q_{l}(x)$, that is, if $\Psi$ is not equivalent to $\bar{\Psi}$, then we also have associated to $\Psi$ a label $\delta \in\{+1,-1\}$. The modules $\Psi$ are parametrized by their polynomials $Q_{1}(x), \ldots, Q_{l}(x)$ and by their labels $\delta$ (if the latter exist) up to equivalence and similarity [M2, Corollary 4.4.17]. For example, if $\Psi$ is the trivial $\mathrm{Y}\left(\mathfrak{s o}_{n}\right)$-module, then $Q_{1}(x)=\cdots=Q_{l}(x)=1$, and there is no label $\delta$.

In this subsection $\mathfrak{g}=\mathfrak{s o}_{2 m}$, as in Subsection 5.4. In particular, here $\rho_{a}=1-a$ for each index $a=1, \ldots, m$. Suppose that the weight $\lambda+\rho$ is dominant. This condition can be written as the collection of inequalities (5.16) and (5.17). However, now $\kappa_{a}=-l$. Using the definition (2.18) of the weight $\nu$, suppose that each label $\nu_{a} \in\{0,1, \ldots, n\}$.

At the end of Subsection 4.4 we observed that for $\mathfrak{g}=\mathfrak{s o}_{2 m}$ our intertwining operators (4.58) and (4.59) are the same. Denote by $N$ the quotient by the kernel of this operator. By Proposition 4.2 and Corollary 4.9. $N$ is either an irreducible $\mathrm{Y}\left(\mathfrak{s o}_{n}\right)$-module or splits into a direct sum of two irreducible nonequivalent $\mathrm{Y}\left(\mathfrak{s o}_{n}\right)$ modules. In the latter case, our $N$ is irreducible as a module over the algebra $\mathrm{O}_{n} \ltimes \mathrm{Y}\left(\mathfrak{s o}_{n}\right)$ by Proposition 4.8 .

Due to the $\mathrm{O}_{n}$-equivariance of the homomorphism $\mathrm{X}\left(\mathfrak{s o}_{n}\right) \rightarrow \mathrm{A}^{\mathrm{O}_{2 m}}$, for any $v \in \mathrm{O}_{n}$ pulling the action of $\mathrm{Y}\left(\mathfrak{s o}_{n}\right)$ on $N$ back through the automorphism (4.52) amounts to pulling that action forward through the automorphism of $X \mapsto v X v^{-1}$ of the algebra End $N$. Let us apply this observation to the element $v$ used at the beginning of this subsection. If the $\mathrm{Y}\left(\mathfrak{s o}_{n}\right)$-module $N$ is irreducible, then it is of the form $\Psi$, where $\Psi$ and $\bar{\Psi}$ are equivalent. If $N$ splits to a direct sum of two irreducible nonequivalent submodules and one of them is denoted by $\Psi$, the other submodule is equivalent to $\bar{\Psi}$.

As in Subsection 5.4, the definition (4.10) of the comultiplication on $\mathrm{Y}\left(\mathfrak{g l}_{n}\right)$ implies that the vector (4.57) is highest for the twisted Yangian $\mathrm{Y}\left(\mathfrak{s o}_{n}\right)$. Here we again used Lemma 5.2. Suppose the weight $\nu$ satisfies the inequalities (5.18) and 
(5.19). Note that the weights $\kappa$ used here and in Subsection 5.4 to determine $\nu$ are different. It is the form of the inequalities (5.18) and (5.19) for $\nu$ that is the same.

We have already noted that for $\sigma=\sigma_{0}$ and $\omega=\tau_{m} \sigma_{0}$ our operator (4.53) is invertible. Due to the dominance of the weight $\lambda+\rho$, Proposition 4.6 now implies that the image of the vector (4.57) in the quotient $N$ is not zero. This image is going to be our vector $\psi$. In particular, it will satisfy (5.21) and (5.22) for certain polynomials $Q_{1}(x), \ldots, Q_{l}(x)$. Lemma 5.2 implies that each of these polynomials is multiplicative with respect to the $m$ tensor factors of the vector (4.57). But in the case $m=1$, these polynomials are transparent from the relations given at the very end of Subsection 5.2. Thus we get

Theorem 5.5. Put $n=2 l$. Let $\lambda_{1}, \ldots, \lambda_{m}$ satisfy (5.16), (5.17) while the labels

$$
\nu_{1}=\lambda_{1}-\mu_{1}+l, \ldots, \nu_{m}=\lambda_{m}-\mu_{m}+l
$$

belong to the set $\{0,1, \ldots, n\}$ and satisfy the conditions (5.18), (5.19). Then the quotient by the kernel of our intertwining operator (4.58) is either a nonzero irreducible self-conjugate $\mathrm{Y}\left(\mathfrak{s o}_{n}\right)$-module or splits into a direct sum of two nonequivalent nonzero irreducible $\mathrm{Y}\left(\mathfrak{s o}_{n}\right)$-modules conjugate to each other. For every $k=$ $1, \ldots, l-1$, polynomial $Q_{k}(x)$ of any of the irreducible modules is the product of the sums $x+\mu_{a}+\rho_{a}$ taken over all indices a such that $\nu_{a}=k$ and of the differences $x-\mu_{a}-\rho_{a}$ taken over all indices a such that $\nu_{a}=n-k$. The polynomial $Q_{l}(x)$ of any of the irreducible modules is the product of the differences $x^{2}-\left(\mu_{a}+\rho_{a}\right)^{2}$ taken over all indices a such that $\nu_{a}=l$. The splitting occurs if and only if $\mu_{a}+\rho_{a}=0$ for at least one index a such that $\nu_{a}=l$.

In view of (5.23), the splitting in Theorem 5.5 occurs if and only if $\lambda_{a}+\rho_{a}=0$ and $\mu_{a}+\rho_{a}=0$ simultaneously for at least one index $a$. If $\nu_{1}=\cdots=\nu_{m}=0$, then both the source and the target $\mathrm{Y}\left(\mathfrak{s o}_{n}\right)$-modules in (4.58) are trivial for any $\mu$. If moreover $\lambda+\rho$ is dominant, then by Proposition 4.6 our operator (4.58) is the identity map $\mathbb{C} \rightarrow \mathbb{C}$.

Now let us take any sequence of $l$ monic polynomials $Q_{1}(x), \ldots, Q_{l}(x)$ with complex coefficients, such that the polynomial $Q_{l}(x)$ is even. Let $m$ be the sum of the degrees of $Q_{1}(x), \ldots, Q_{l-1}(x)$ plus half of the degree of $Q_{l}(x)$. Suppose that $m>0$. Let $\lambda$ and $\mu$ be any weights of $\mathfrak{s o}_{2 m}$ such that the corresponding labels (5.23) belong to the set $\{1, \ldots, n-1\}$ and such that the given polynomials $Q_{1}(x), \ldots, Q_{l}(x)$ are obtained from $\lambda$ and $\mu$ as in Theorem 5.5. We do not yet impose any other conditions on $\lambda$ and $\mu$.

Then $\lambda$ and $\mu$ are determined up to permuting the $m$ pairs $\left(\nu_{a}, \mu_{a}+\rho_{a}\right)$, and up to replacing $\left(\nu_{a}, \mu_{a}+\rho_{a}\right)$ by $\left(n-\nu_{a},-\mu_{a}-\rho_{a}\right)$ for any number of indices $a$. Equivalently, the weights $\lambda$ and $\mu$ are determined up to a permutation of the $m$ pairs $\left(\lambda_{a}+\rho_{a}, \mu_{a}+\rho_{a}\right)$, and up to replacing $\left(\lambda_{a}+\rho_{a}, \mu_{a}+\rho_{a}\right)$ by $\left(-\lambda_{a}-\rho_{a},-\mu_{a}-\rho_{a}\right)$ for any number of indices $a$. Thus $\lambda$ and $\mu$ are determined up to the (simultaneous) shifted action of the group $\mathfrak{R}$ of $\mathfrak{s o}_{2 m}$ on them, as on elements of $\mathfrak{t}^{*}$. Using only the action of the subgroup $\mathfrak{S} \subset \mathfrak{R}$, we can choose $\lambda$ to satisfy the conditions (5.16), (5.17) so that the weight $\lambda+\rho$ is dominant.

By using only the shifted action on $\mu$ of those elements of the subgroup $\mathfrak{S} \subset \mathfrak{R}$ which leave $\lambda$ invariant, we can choose $\mu$ so that the conditions (5.18), (5.19) are satisfied. The arguments are the same as at the end of Subsection 5.4, and we do not repeat them here. 
Now consider our intertwining operator (4.58) corresponding to these weights $\lambda$ and $\mu$. Due to Theorem 5.5. the quotient by the kernel of this operator is either a nonzero irreducible integrable $\mathrm{Y}\left(\mathfrak{s o}_{n}\right)$-module or splits into a direct sum of two nonequivalent irreducible integrable $\mathrm{Y}\left(\mathfrak{s o}_{n}\right)$-modules. To any of the irreducible modules we associate the given polynomials $Q_{1}(x), \ldots, Q_{l}(x)$. If the quotient is irreducible, there is no label $\delta$ associated to it. If the quotient splits into two irreducible modules, they have the labels $\delta=1$ and $\delta=-1$ associated to them. Therefore up to equivalence and similarity, every nonzero irreducible integrable finite-dimensional $\mathrm{Y}\left(\mathfrak{s o}_{n}\right)$-module arises either as such a quotient, or as one of its two direct summands. Note that the choice $\theta=-1$ here is essential.

\section{ACKNOWLEDGMENTS}

We are grateful to Ernest Vinberg for collaborating with us on KNV]. The present work began when we visited MPIM, and continued when we visited IHES. We are grateful to the staff of both institutes for their kind help and hospitality. The first author was supported by the RFBR grant 08-01-00392, joint grant 0901-93106, interdisciplinary grant 09-01-12185-ofi-m, and the grant for Support of Scientific Schools 3036-2008-2. The second author was supported by the EPSRC grant C511166.

\section{REFERENCES}

[AK] T. Akasaka and M. Kashiwara, Finite-dimensional representations of quantum affine algebras, Publ. Res. Inst. Math. Sci. 33 (1997), 839-867. MR1607008 (99d:17017)

[AS] T. Arakawa and T. Suzuki, Duality between $\mathfrak{s l}_{n}(\mathbb{C})$ and the degenerate affine Hecke algebra, J. Algebra 209 (1998), 288-304. MR:1652134(99h:17005)

[AST] R. Asherova, Y. Smirnov and V. Tolstoy, A description of certain class of projection operators for complex semisimple Lie algebras, Math. Notes 26 (1980), 499-504.

[B] N.Bourbaki, Groupes et algèbres de Lie IV-VI, Hermann, Paris, 1968. MR0240238 $(39: 1590)$

[BK] J.Brundan and A. Kleshchev, Representations of shifted Yangians and finite $W$-algebras, Mem. Amer. Math. Soc. 196 (2008), 1-107. MR2456464 (2009i:17020)

[C] V. Chari, Braid group actions and tensor products, Internat. Math. Res. Notices (2002), 357-382. MR 1883181 (2003a:17014)

[CP] V. Chari and A. Pressley, Fundamental representations of Yangians and singularities of R-matrices, J. Reine Angew. Math. 417 (1991), 87-128. MR1103907 (92h:17010)

[C1] I. Cherednik, Factorized particles on the half-line and root systems, Theor. Math. Phys. 61 (1984), 977-983. MR774205 (86g:81148)

[C2] I. Cherednik, A new interpretation of Gelfand-Tzetlin bases, Duke Math. J. 54 (1987), 563-577. MR899405 (88k:17005)

[D1] V. Drinfeld, Degenerate affine Hecke algebras and Yangians, Funct. Anal. Appl. 20 (1986), 56-58. MR831053 (87m:22044)

[D2] V.Drinfeld, A new realization of Yangians and quantized affine algebras, Soviet Math. Dokl. 36 (1988), 212-216. MR914215 (88j:17020)

[EV] P. Etingof and A. Varchenko, Dynamical Weyl groups and applications, Adv. Math. 167 (2002), 74-127. MR1901247(2003d:17004)

[H] Harish-Chandra, Representations of semisimple Lie groups II, Trans. Amer. Math. Soc. 76 (1954), 26-65. MR0058604 (15:398a)

[H1] R. Howe, Remarks on classical invariant theory, Trans. Amer. Math. Soc. 313 (1989), 539-570. MR986027 (90h:22015a)

[H2] R. Howe, Perspectives on invariant theory: Schur duality, multiplicity-free actions and beyond, Israel Math. Conf. Proc. 8 (1995), 1-182. MR.1321638(96e:13006) 
[K] S. Khoroshkin, Extremal projector and dynamical twist, Theoret. Math. Phys. 139 (2004), 582-597. MR 2076916 (2005f:17016)

[KN1] S. Khoroshkin and M. Nazarov, Yangians and Mickelsson algebras I, Transformation Groups 11 (2006), 625-658. MR2278142 (2008d:17016)

[KN2] S. Khoroshkin and M. Nazarov, Yangians and Mickelsson algebras II, Moscow Math. J. 6 (2006), 477-504. MR2274862(2008d:17017)

[KN3] S. Khoroshkin and M. Nazarov, Twisted Yangians and Mickelsson algebras I, Selecta Math. 13 (2007), 69-136 and 14 (2009), 321. MR2330588 (2009d:17021)

[KN4] S. Khoroshkin and M. Nazarov, Twisted Yangians and Mickelsson algebras II, St. Petersburg Math. J. 21 (2010), 111-161. MR2553055

[KNV] S. Khoroshkin, M. Nazarov and E. Vinberg, A generalized Harish-Chandra isomorphism, Adv. Math. 226 (2011), 1168-1180. MR.2737780

[KO] S. Khoroshkin and O. Ogievetsky, Mickelsson algebras and Zhelobenko operators, J. Algebra 319 (2008), 2113-2165. MR 2394693 (2009a:16040)

[KRS] P. Kulish, N. Reshetikhin and E. Sklyanin, Yang-Baxter equation and representation theory, Lett. Math. Phys. 5 (1981), 393-403. MR649704 (83g:81099)

[LM] J. Lepowski and G. McCollum, On the determination of irreducible modules by restriction to a subalgebra, Trans. Amer. Math. Soc. 176 (1973), 45-57. MR0323846 (48:2201)

[M] J. Mickelsson, Step algebras of semi-simple subalgebras of Lie algebras, Rep. Math. Phys. 4 (1973), 307-318. MR0342057 (49:6803)

[M1] A. Molev, Skew representations of twisted Yangians, Selecta Math. 12 (2006), 1-38. MR 2244262(2007h:17013)

[M2] A. Molev, Yangians and classical Lie algebras, Amer. Math. Soc., Providence, RI, 2007. MR2355506 (2008m:17033)

[MO] A. Molev and G. Olshanski, Centralizer construction for twisted Yangians, Selecta Math. 6 (2000), 269-317. MR:1817615 (2002j:17013)

[MTZ] A. Molev, V. Tolstoy and R. Zhang, On irreducibility of tensor products of evaluation modules for the quantum affine algebra, J. Phys. A 37 (2004), 2385-2399. MR2045932 (2005g:17034)

[N] M. Nazarov, Representations of twisted Yangians associated with skew Young diagrams, Selecta Math. 10 (2004), 71-129. MR2061224 (2005e:17026)

[NT1] M. Nazarov and V. Tarasov, On irreducibility of tensor products of Yangian modules, Internat. Math. Res. Notices (1998), 125-150. MR1606387 (99b:17014)

[NT2] M. Nazarov and V. Tarasov, On irreducibility of tensor products of Yangian modules associated with skew Young diagrams, Duke Math. J. 112 (2002), 343-378. MR1894364 (2003b:17021)

[O1] G. Olshanski, Extension of the algebra $U(g)$ for infinite-dimensional classical Lie algebras $g$, and the Yangians $Y(g l(m))$, Soviet Math. Dokl. 36 (1988), 569-573. MR936073 (89g:17017)

[O2] G. Olshanski, Twisted Yangians and infinite-dimensional classical Lie algebras, Lecture Notes Math. 1510 (1992), 103-120. MR.1183482 (93h:17039)

[S] E. Sklyanin, Boundary conditions for integrable quantum systems, J. Phys. A 21 (1988), 2375-2389. MR953215 (89h:81258)

[T1] V. Tarasov, Structure of quantum L-operators for the R-matrix of the XXZ-model, Theor. Math. Phys. 61 (1984), 1065-1071. MR778541 (87c:82023)

[T2] V.Tarasov, Irreducible monodromy matrices for the $R$-matrix of the $X X Z$-model and lattice local quantum Hamiltonians, Theor. Math. Phys. 63 (1985), 440-454. MR800062 (87d:82022)

[TV1] V.Tarasov and A. Varchenko, Difference equations compatible with trigonometric KZ differential equations, Internat. Math. Res. Notices (2000), 801-829. MR.1780748 (2001k:32025)

[TV2] V. Tarasov and A. Varchenko, Duality for Knizhnik-Zamolodchikov and dynamical equations, Acta Appl. Math. 73 (2002), 141-154. MR1926498 (2003h:17024)

[T] J. Tits, Normalisateurs de tores I. Groupes de Coxeter étendu, J. Algebra 4 (1966), 96116. MR 0206117 (34:5942)

[W] H. Weyl, Classical Groups, their Invariants and Representations, Princeton University Press, Princeton, 1946. MR,1488158 (98k:01049) 
[Z1] D. Zhelobenko, Extremal cocycles on Weyl groups, Funct. Anal. Appl. 21 (1987), 183-192. MR 911771 (89g:17007)

[Z2] D. Zhelobenko, Extremal cocycles and generalized Mickelsson algebras over reductive Lie algebras, Math. USSR Izvestiya 33 (1989), 85-100. MR.966983 (89m:17021)

Institute for Theoretical and Experimental Physics, Moscow 117259, Russia - And Department of Mathematics, Higher School of Economics, Moscow 117312, Russia

E-mail address: khor@itep.ru

Department of Mathematics, University of York, York YO10 5DD, England

E-mail address: mln1@york.ac.uk 